\title{
SULFUR VULCANIZATION OF NATURAL RUBBER FOR BENZOTHIAZOLE ACCELERATED FORMULATIONS: FROM REACTION MECHANISMS TO A RATIONAL KINETIC MODEL
}

\author{
Prasenjeet Ghosh ${ }^{+}$Santhoji Katare, Priyan Patkar \\ James M. Caruthers, * Venkat Venkatasubramanian \\ School of Chemical Engineering, Purdue University \\ West LafayetTe, IN 47906-1283
}

\section{KENNETH A. WALKER}

Chemical Products Business Unit, Caterpillar, Inc. P.O. BoX 400, MossviLle, IL 61552-4000

\begin{abstract}
The chemistry of accelerated sulfur vulcanization is reviewed and a fundamental kinetic model for the vulcanization process is developed. The vulcanization of natural rubber by the benzothiazolesulfenamide class of accelerators is studied, where 2-(morpholinothio) benzothiazole (MBS) has been chosen as the representative accelerator. The reaction mechanisms that have been proposed for the different steps in vulcanization chemistry are critically evaluated with the objective of developing a holistic description of the governing chemistry, where the mechanisms are consistent for all reaction steps in the vulcanization process. A fundamental kinetic model has been developed for accelerated sulfur vulcanization, using population balance methods that explicitly acknowledge the polysulfidic nature of the crosslinks and various reactive intermediates. The kinetic model can accurately describe the complete cure response including the scorch delay, curing and the reversion for a wide range of compositions, using a single set of rate constants. In addition, the concentration profiles of all the reaction intermediates as a function of polysulfidic lengths are predicted. This detailed information obtained from the population balance model is used to critically examine various mechanisms that have been proposed to describe accelerated sulfur vulcanization. The population balance model provides a quantitative framework for explicitly incorporating mechanistically reasonable chemistry of the vulcanization process.
\end{abstract}

\section{CONTENTS}

I. Introduction . . . . . . . . . . . . . . . . . . . . . . . .593

II. Kinetic Models for Accelerated Sulfur Vulcanization _. . . . . . . . . . . . 597

A. Chemistry Preliminaries .........................597

B. Empirical and Other Limited Kinetic Models .............. . .599

C. Mechanistic Kinetic Models .....................600

III. Chemistry of Accelerated Sulfur Vulcanization . . . . . . . . . . . . . .603

A. Accelerator Chemistry .........................603

1. Formation of MBTS from MBS ...............605

2. Reaction of Sulfur with MBTS and Other Accelerator Polysulfides . .609

3. Exchange Reaction Among Accelerator-Polysulfides ........613

4. Effect of $\mathrm{ZnO}$ - Formation of Zinc-Accelerator Complexes . .614

B. Crosslinking Chemistry ..............................

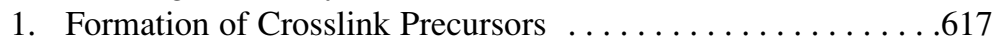

2. Conversion of Crosslink Precursors to Crosslinks .......620

3. Scorch Delay Reactions ......................623

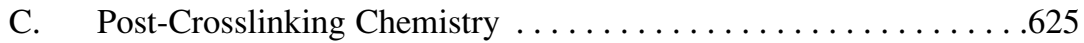

1. Crosslink Desulfuration .....................625

2. Crosslink Degradation and Main-Chain-Modification ......627

\footnotetext{
* Corresponding author. Ph: 765-494-6625; Fax: 765-494-0805; email: caruther@ecn.purdue.edu

+ Current address: ExxonMobil Research and Engineering, Paulsboro, NJ 08066
} 


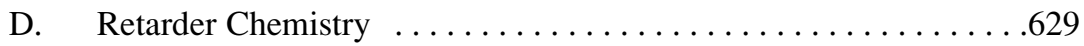

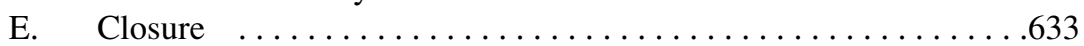

IV. Population Balance Equations for Sulfur Vulcanization $\ldots \ldots \ldots \ldots 635$

A. PBEs for Model-I: Sulfur Pick-up as $\mathrm{S}_{8} \ldots \ldots \ldots \ldots \ldots \ldots \ldots 60.6 \ldots$

B. PBEs for Model-II: Sequential Sulfur Pick-up . . . . . . . . . . .645

C. Incorporation of Retarder in the PBEs . . . . . . . . . . . . .648

V. Estimation of Looping Probability Using Conformational Analysis . . . . .648

A. Conformational Analysis of Sulfur and Isoprene Chains ......650

B. Estimation of Looping Probabilities . . . . . . . . . . . . . .652

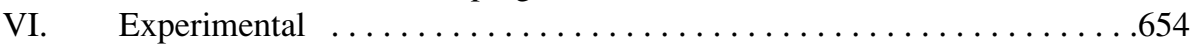

VII. Results ....................................655

A. Predictions for Model-I: Sulfur Pick-up as $S_{8} \ldots \ldots \ldots \ldots \ldots 65$

B Predictions for Model-II: Sequential Sulfur Pick-up . . . . . . . . .664

C. Error and Sensitivity Analysis for Estimates of Rate Constants . . .679

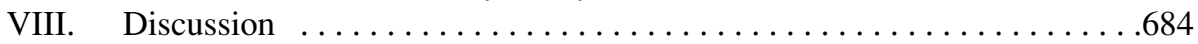

IX. Acknowledgements . . . . . . . . . . . . . . . . . . . .689

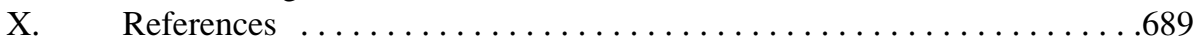

\section{INTRODUCTION}

The discovery of sulfur vulcanization of rubber by Goodyear and independently by Hancock more than 150 years ago was the genesis of one of the important classes of engineering materials. A vigorous research effort continues today to develop a fundamental understanding of this complex chemical process. Although the initial work by Goodyear and Hancock was concerned with the addition of sulfur to natural rubber, various accelerators, activators and retarders have been subsequently employed in order to better control the various aspects of the rate and extent of the vulcanization process. More recently, mixed accelerator systems like thiuram-sulfenamides have been developed to allow additional control over the vulcanization process. It is not surprising that the cure chemistry is complicated for these complex mixtures of elastomer, sulfur, accelerators, activators, and retarders. Notwithstanding the considerable research in studying the vulcanization process, a fundamental understanding is far from complete as clearly stated by two pioneers in the field:

"Perhaps nowhere in chemistry is there encountered a field which even in its literature alone shows so many uncertainties and (possibly only apparent) contradictions as that of the vulcanization of rubber." Scheele ${ }^{1}$ and

"Whilst it has long been appreciated, albeit intuitively, that sulfur vulcanization is a very complex chemical process, the actual complexity... is probably far in excess of what has ever been envisaged." Bateman et $_{\text {al. }}{ }^{2}$

Moreover, in a recent review Nieuwenhuizen, et al. ${ }^{3}$ state:

"It must be considered remarkable that despite all the efforts and progress in the field of vulcanization during the past decade, one has to conclude that the statements of Scheele and Bateman, made 30 to 40 years ago, are still true to a great extent."

Although the situation may not be quite as bleak as that indicated above, a complete understanding of the vulcanization process clearly remains a significant scientific challenge. Over the last several decades, significant progress has been made in unraveling the mechanisms of many of the individual steps in the vulcanization process; however, the overwhelming majority of vulcanization studies to date have focused on identifying qualitative aspects of the underlying reaction mechanisms. In contrast, mechanistically reasonable, quantitative models of the vulcanization kinetics are not currently available. The objective of this paper is twofold: first, to critically 
review the various reaction mechanisms that have been proposed for the sulfenamide class of accelerators in order to develop a self-consistent description for the vulcanization process; and second, to develop a fundamental framework for describing the kinetics of sulfenamide accelerated sulfur vulcanization. Although the focus of this paper is on sulfenamide accelerated systems, the quantitative approach that will be developed will, in principle, be valid for a wide range of accelerator systems.

A fundamental description of vulcanization kinetics must obviously acknowledge the details of the underlying chemical mechanisms and thus the composition of the formulation. In this paper, we will demonstrate the applicability of this fundamental kinetic approach for a particular system - the vulcanization of natural rubber accelerated with 2-(morpholinothio) benzothiazole (i.e. MBS), which is a technologically important member of the benzothiazolesulfenamide class of accelerators. A list of common accelerators is given in Table I, where they have been categorized into benzothiazoles, benzothiazolesulfenamides, thiurams, and dithiocarabamates. The various abbreviations that will be used throughout this paper are given in Table II. Although the chemical structures of the various accelerator systems shown in Table I are different, the basic features of the accelerators are similar; specifically, the accelerator is composed of one or two sulfur atoms between a pair of organic end groups. Because of this similarity, we believe a fundamental kinetic framework for vulcanization will be valid for a wide variety of accelerator and elastomer systems. We will discuss how the kinetic framework developed in this paper can be extended for accelerator systems given in Table I as well as for various elastomers, although we will only provide full details for the MBS accelerated natural rubber system in this paper. Our specific objective is to quantitatively predict the evolution of the crosslink density during the vulcanization process as measured via an Oscillating Disk Rheometer (ODR) as a function of the initial composition and temperature. Furthermore, we also want to predict the evolution of key intermediate species. This kinetic modeling objective is obviously substantial; however, we believe that it is the most appropriate path for developing a fundamental kinetic description of accelerated sulfur vulcanization. 
SULFUR VULCANIZATON OF NATURAL RUBBER FOR BENZOTHIAZOLE

TABLE I

COMmon AcCelerators Used in Sulfur Vulcanization

\begin{tabular}{|c|c|c|}
\hline Compound & Abbreviation & Structure \\
\hline \multicolumn{3}{|l|}{ Benzothiazoles } \\
\hline 2-mercaptobenzothiazoles & MBT & \\
\hline 2,2'-dithiobenzothiazole & MBTS & \\
\hline \multicolumn{3}{|l|}{ Benzothiazolesulfenamides } \\
\hline N-cyclohexylbenzothiazole-2-sulfenamide & CBS & \\
\hline $\mathrm{N}$-t-butylbenzothiazole-2-sulfenamide & TBBS & \\
\hline 2-morpholinothiobenzothiazole & MBS & \\
\hline \multicolumn{3}{|l|}{$\begin{array}{l}\text { N-dicyclohexylbenzothiazole-2- } \\
\text { sulfenamide }\end{array}$} \\
\hline \multicolumn{3}{|l|}{ Thiurams } \\
\hline Tetramethylthiuram monosulfide & TMTM & $\begin{array}{cc}\mathrm{CH}_{3}-\underset{1}{\mathrm{~N}}-\mathrm{C}-\mathrm{S}-\mathrm{C}-\mathrm{C}-\underset{\mathrm{N}}{\mathrm{N}}-\mathrm{CH}_{3} \\
\mathrm{CH}_{3} & \mathrm{CH}_{3}\end{array}$ \\
\hline Tetramethylthiuram disulfide & TMTD & 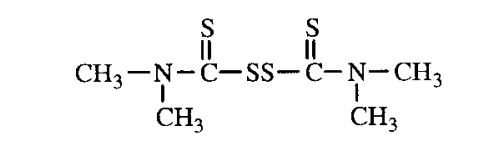 \\
\hline \multicolumn{3}{|l|}{ Dithiocarbamates } \\
\hline Zinc Dimethyldithiocarbamate & ZDMC & 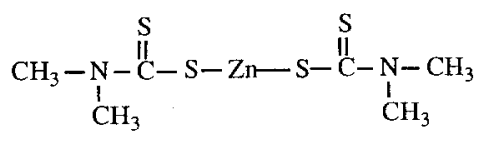 \\
\hline Zinc Diethyldithiocarbamate & ZDEC & 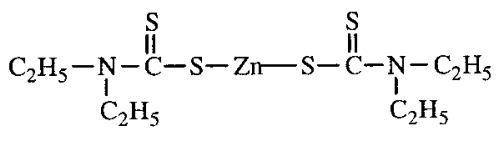 \\
\hline
\end{tabular}


TABLE II

List OF ABBREVIATIONS AND SyMbOLS

\begin{tabular}{|c|c|}
\hline Abbreviation & Explanation \\
\hline ODR & Oscillating Disk Rheometer \\
\hline $\mathrm{ES}(\mathrm{P}) \mathrm{R}$ & Electron Spin (Paramagnetic) Resonance \\
\hline HPLC & High Pressure Liquid Chromatography \\
\hline $\mathrm{MCV}$ & Model Compound Vulcanization \\
\hline MAS & Magic Angle Spinning \\
\hline FT & Fourier Transform \\
\hline $\mathrm{Bt}$ & Benzothiazole group, $\mathrm{C}_{7} \mathrm{H}_{4} \mathrm{NS}$ \\
\hline MBT or BtSH & 2-mercaptobenzothiazole \\
\hline MBTM or Bt-S-Bt & 2-bisbenzothiazole-2,2'-monosulfide \\
\hline MBTS or Bt-SS-Bt & 2-bisbenzothiazole-2,2'-disulfide \\
\hline MBTP or BtS-S ${ }_{x}-S B t$ & 2-bisbenzothiazole-2,2'-polysulfide \\
\hline $\mathrm{BtS}-\mathrm{Zn}-\mathrm{S}_{\mathrm{x}}-\mathrm{SBt}$ & Zinc-Mercaptobenzothiazole Polysulfides \\
\hline MBS or Bt-S-NR 2 & 2-morpholinothiobenzothiazole \\
\hline $\mathrm{MDB}$ or $\mathrm{Bt}-\mathrm{SS}-\mathrm{NR}_{2}$ & 2-morpholinodithiobenzothiazole \\
\hline TBBS & N-t-butylbenzothiazole-2-sulfenamide \\
\hline ZMBT & Zinc bis (mercaptobenzothiazolate) \\
\hline TMTD & Tetramethylthiuram disulfide \\
\hline TMTP & Tetramethylthiuram polysulfide \\
\hline ZDMC & Zinc bis (dimethyldithiocarbamate) \\
\hline ZDEC & Zinc bis (diethyldithiocarbamate) \\
\hline $\mathrm{R}-\mathrm{S}_{\mathrm{x}} \cdot$ & Alkenyl Persulfenyl Radical \\
\hline $\mathrm{Bt}_{-} \mathrm{S}_{\mathrm{x}} \cdot$ & Benzothiazole Terminated Polysulfidic Radical \\
\hline $\mathrm{RH}$ & Rubber \\
\hline TME & 2-3-dimethyl-2-butene \\
\hline CBS & N-cyclohexylbenzothiazole-2-sulfenamide \\
\hline CBP & N-cyclohexylbenzothiazole Polysulfide \\
\hline CTP & $\mathrm{N}$-(cyclohexylthio) pthalimide \\
\hline CDB & 2-cyclohexyldithiobenzothiazole \\
\hline
\end{tabular}

This paper will be organized as follows. In Section II, we will briefly review and critically evaluate existing kinetic models that have been proposed to describe vulcanization chemistry, where it will become apparent that a more fundamental description of the underlying chemical reaction mechanisms will be required. In Section III, an extensive review of the reaction mechanisms that have been proposed for benzothiazolesulfenamide accelerated systems will be provided. In Section IV, the population balance modeling framework, which is the key original contribution of this paper, will be developed in order to describe the kinetics of vulcanization and the governing set of differential equations presented. Section V will have a slightly different focus, employing conformational analysis of the polysulfidic chains to provide insights in the formation of cyclic sulfides during the reversion part of the vulcanization process. The experimental methods we have employed to measure the evolution of crosslink density for various formulations will be described in Section VI. In Section VII, we will present the predictions of the kinetic model developed in this paper along with a rigorous nonlinear statistical analysis of the 
kinetic parameters that are determined from the experimental data. Finally, in Section VIII, we will critically discuss the implications of the proposed kinetic framework and how quantitative kinetic modeling can provide insight into the mechanisms for accelerated vulcanization.

This paper has benefited from the insights provided by previous review articles on different aspects of vulcanization. Some of the more notable reviews in chronological order include the early work by Bateman et al. ${ }^{2}$ who provided a comprehensive review on the chemistry of sulfur vulcanization; Porter ${ }^{4,5}$ who subsequently updated the Bateman et al. review; Scheele ${ }^{6}$ and Saville and Watson ${ }^{7}$ who summarized the theoretical and experimental investigations on network characterization; and, Trivette $e t a l .{ }^{8}$ who discussed the chemistry of retarders in sulfur vulcanization. In the 1980's, Morita ${ }^{9}$ highlighted the importance of S-N compounds as accelerators as well as retarders; while McCleverty ${ }^{10-14}$ pioneered critical studies of the inorganic chemistry of zinc-accelerator complexes. More recently, Kresja and Koenig ${ }^{15}$ summarized additional details of the chemistry of accelerated and unaccelerated vulcanization, while Nieuwenhuizen et al. ${ }^{3}$ exhaustively reviewed the mechanistic details of the chemistry of thiurams and dithiocarbamates. We hope that this paper may in some small way add to this distinguished collection of publications.

\section{KINETIC MODELS FOR ACCELERATED SULFUR VULCANIZATION}

\section{A. CHEMISTRY PRELIMINARIES}

Before discussing specific kinetic models for sulfur vulcanization, it is useful to summarize the generally accepted mechanism of accelerated sulfur vulcanization. Although important differences remain, there is wide spread agreement about the basic steps in the vulcanization reactions as illustrated schematically in Figure $1 .{ }^{16}$ The vulcanization reactions can be divided into three sub-categories: (i) accelerator chemistry which involves the reactions leading to the formation of an active-sulfurating agent; (ii) crosslinking chemistry which includes reactions leading to the formation of crosslinks; and (iii) post-crosslinking chemistry which involves reactions that lead to crosslink shortening and crosslink degradation. The first step in accelerated sulfur vulcanization is the formation of an active accelerator complex via reaction of the accelerator and the activator, which subsequently reacts with molecular sulfur to form a distribution of sulfurating species. These activated sulfurating species then react with an unsaturated site, in particular an allylic carbon, on the rubber chain to form crosslink precursors, which are accelerator-terminated polysulfidic pendant groups attached to the rubber chain. These crosslink precursors subsequently react with additional unsaturated sites on the rubber chain resulting in polysulfidic crosslinks. The polysulfidic crosslinks may eventually (i) desulfurate over longer times to form shorter crosslinks, or (ii) degrade to cyclic sulfides or other main-chain modifications, which can cause the long-term deterioration of vulcanizate properties. The term "crosslinks" includes various chemical structures as shown schematically in Figure 2 for sulfur vulcanized networks. The distinction between mono, di and polysulfidic crosslinks is important, since the polysulfidic length distribution of crosslinks affects thermal stability and hence the final properties of the vulcanizates. In addition to the chemical crosslinks, Figure 2 also indicates additional chemical structures that may be introduced during vulcanization, like cyclic sulfides, pendant accelerator residues, vicinal crosslinks and main-chain modifications.

The simplest experiment to characterize vulcanization kinetics is the evolution of crosslinks, which is commonly, called the cure-curve, and can be measured using an ODR. An ODR measures the torque, or equivalently the shear modulus, as a function of time when the vulcanizate cures, where it is implicitly assumed that the modulus is proportional to the evolving concentration of crosslinks. A typical schematic of the cure-curve obtained from an ODR is shown in Figure 3. The cure curve exhibits three main regions: The first region is the scorch delay or the 
induction region, and it is during this phase of the vulcanization where the majority of the accelerator chemistry takes place. The second region is associated with the cure reaction, where sulfur crosslinking takes place and the crosslinked network forms. The third and final region of the ODR curve is the postcure region where the modulus can increase, decrease or remain constant depending upon the specific vulcanization system. An increase in modulus is a marching modulus cure that is typically exhibited by neoprene rubber; a decrease in modulus is reversion, which is very common in natural rubber systems; and finally, the cure can result in a constant equilibrium modulus, which is a characteristic of styrene-butadiene systems.

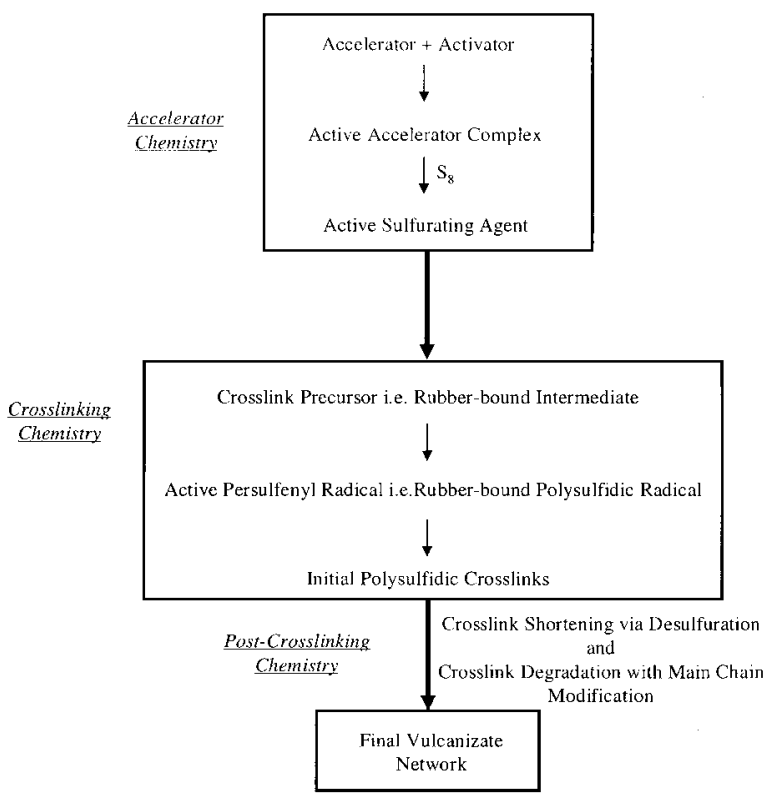

FIG. 1. - General mechanism for accelerated sulfur vulcanization. Mechanism adapted from that of Morrison and Porter. ${ }^{16}$

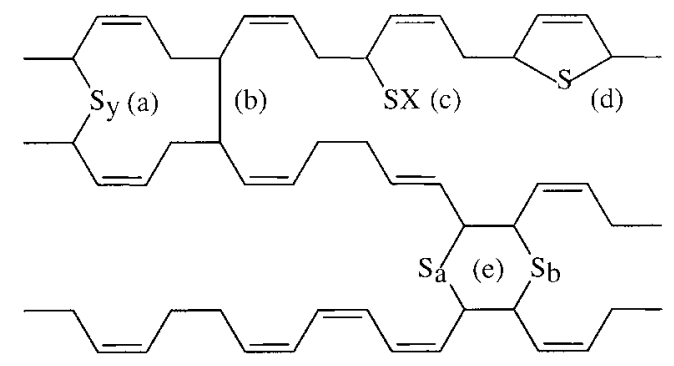

FIG. 2. - Various chemical structures encountered in accelerated sulfur vulcanization. (a) sulfur crosslinks ( $\mathrm{y}=1$ mono, $\mathrm{y}=2$ di and $\mathrm{y}>2$ polysulfide crosslinks); (b) carbon-carbon crosslink;

(c) pendant accelerator sulfide where $\mathrm{X}$ is the accelerator moiety; (d) cyclic sulfide; and

(e) vicinal crosslinks that have junction points at common olefin chains and constitute only one elastically effective crosslink. Figure adapted from Nieuwenhuizen et al. ${ }^{3}$ 


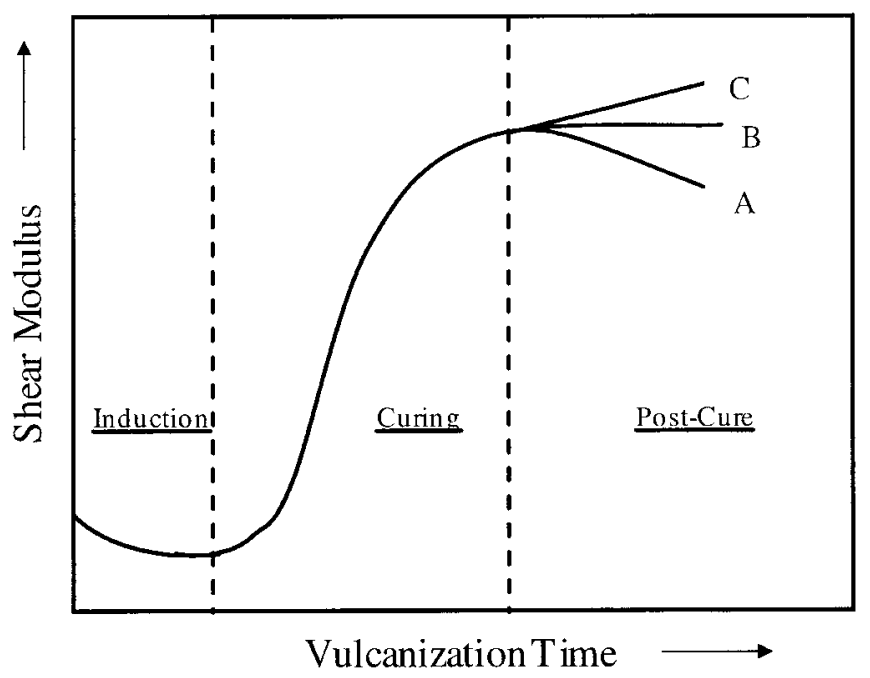

FIG. 3. - A typical rheometer cure curve obtained from an Oscillating Disk Rheometer for accelerated sulfur vulcanization. Curve A: Cure to maximum torque with reversion. Curve B: Cure to equilibrium torque. Curve $\mathrm{C}$ : Cure with no equilibrium or maximum torque.

With this brief background, it is now possible to discuss the different kinetic models that have been proposed to describe vulcanization chemistry. A minimal requirement of any such model is to at least describe the ODR data for isothermal conditions by acknowledging the basic chemistry outlined above. Depending upon the basic premise underlying the model, we have classified the different models in two classes: (i) empirical and other limited kinetic models and (ii) mechanistic kinetic models.

\section{B. EMPIRICAL AND OTHER LIMITED KINETIC MODELS}

Empirical models are regression models that fit the data assuming a particular functional form, where the parameters are estimated from the experimental data using non-linear parameter estimation procedures. A number of empirical models that have been used for modeling sulfur vulcanization are given in Table III. In all these models, the degree of cure $\alpha$ increases monotonically from zero, at the initiation of the cure, to one, if the cure is continued for a sufficiently long time. These empirical models are able to capture the induction and the curing portion of the vulcanization process; however, since in the limit of long times the degree of cure approaches one, these models are not able to even curve-fit the reversion phase of the vulcanization process. Moreover, these empirical models fail to acknowledge the underlying molecular mechanisms and neither do they describe how the kinetics of vulcanization depends upon the composition of the system. Although these models may provide value in describing the shape of the ODR cure curve, they contain no fundamental information. For these reasons, we will not further consider these empirical models. 


\begin{tabular}{|c|c|c|}
\hline & $\begin{array}{c}\text { TABle III } \\
\text { EMPIRICAL KINETIC Models FOR CURE KINETICS } \\
\left(\alpha=\text { DEGREE OF CURE, }=\mathrm{Vu} / \mathrm{Vu}_{\text {max }}\right)\end{array}$ & \\
\hline Model & Solution Characteristics & Reference \\
\hline & $\alpha=1-e^{-k t}(n=1)$ & $\begin{array}{l}\text { nth order } \\
\text { kinetic model }\end{array}$ \\
\hline$\frac{d \alpha}{d t}=k(1-\alpha)^{n}, n \geq 1$ & $\alpha=1-\frac{1}{[1+k t(n-1)]^{1 /(n-1)}},(n>1)$ & \\
\hline$\frac{d \alpha}{d t}=\frac{n}{k} t^{-(n+1)} \alpha^{2}, n \geq 1$ & $\alpha=\frac{k t^{n}}{1+k t^{n}}$ & $\begin{array}{l}\text { Isayev and } \\
\text { Deng (1988) }\end{array}$ \\
\hline $\begin{array}{l}\frac{d \alpha}{d t}=k \alpha^{m}(1-\alpha)^{n} \\
n \geq 1, m<1\end{array}$ & $t=k \int_{0}^{\alpha} \frac{d \alpha}{\alpha^{m}(1-\alpha)^{n}}$ & $\begin{array}{l}\begin{array}{l}\text { Piloyan } \\
\text { (1966) }\end{array}\end{array}$ \\
\hline $\begin{array}{l}\frac{d \alpha}{d t}=\left(k_{1}+k_{2} \alpha^{m}\right)(1-\alpha)^{n} \\
n \geq 1, m<1\end{array}$ & $t=k \int_{0}^{\alpha} \frac{d \alpha}{\left(k_{1}+k_{2} \alpha^{m}\right)(1-\alpha)^{n}}$ & $\begin{array}{l}\text { Kamal and } \\
\text { Ryan (1980) }\end{array}$ \\
\hline
\end{tabular}

Several kinetic models have been proposed that address limited aspects of the underlying chemistry and physics, but do not acknowledge all the mechanistic details of vulcanization given in Figure 1. Jurkowska and Jurkoswski ${ }^{17}$ propose that vulcanization chemistry may be modeled by a set of nine reactions, which includes formation, rearrangement, transformation and degradation of the different crosslinks. However, their set of reactions does not describe the initial accelerator chemistry or the crosslinking chemistry; rather, they only describe the evolution of the polysulfidic crosslinks that are already formed. Taylor $e t$ al. ${ }^{18}$ modeled the vulcanization of poly-1,4-butadiene with a TMTD accelerator, using Monte-Carlo simulations to determine possible reactions in a sphere around the crosslink site. The authors predict the elastic moduli of the vulcanizate as well as details concerning the structure of the network; however, the affect of changes in the accelerator and sulfur concentration on the crosslink density and its distribution is not addressed. Although these models describe features of the crosslink chemistry, they do not address the overall vulcanization process.

\section{MECHANISTIC KINETIC MODELS}

Mechanistic kinetic models need to acknowledge the underlying chemistry as summarized in Figure 1. The most well developed and widely recognized kinetic model to describe accelerated sulfur vulcanization is due to Coran and coworkers, who have provided the first steps towards the development of a rational kinetic framework. In a series of papers ${ }^{19-23}$ investigating the kinetics of benzothiazolesulfenamide accelerators, they proposed the following kinetic mechanism for sulfenamide accelerators:

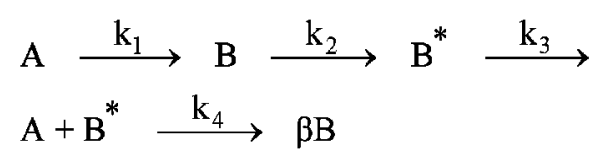

where $\mathrm{A}$ is the accelerator and/or its reaction products with sulfur, $\mathrm{Zn}^{2+}$ etc; $\mathrm{B}$ is the precursor to 
crosslinks, specifically, the accelerator terminated pendant groups; $\mathrm{B}^{*}$ is an activated form of B such as polymeric polythiyl radical; $\mathrm{Vu}$ is the polysulfidic crosslink; and $\alpha$ and $\beta$ are the appropriate stoichiometric coefficients that define the percentage of crosslinks formed from each $\mathrm{B}^{*}$ and the regeneration of $B$ from $B^{*}$ respectively. The rate constants $k_{1}, k_{2}$ and $k_{3}$ capture the kinetics of the initial accelerator chemistry and crosslinking chemistry while the reaction with rate constant $\mathrm{k}_{4}$ accounts for the scorch-delay observed in sulfenamide accelerators. This basic kinetic model was later extended by Ding et al. ${ }^{24}$ to include a competitive side reaction to explain the variation of equilibrium modulus with cure temperature; specifically,

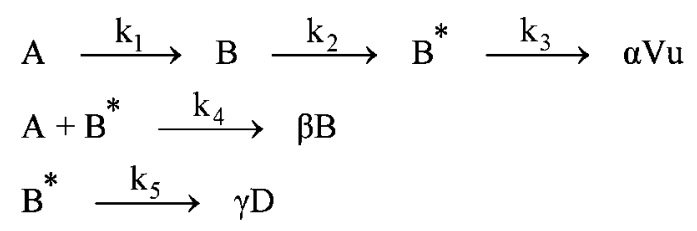

where $\mathrm{D}$ is a dead end species and $\alpha, \beta$ and $\gamma$ are stoichiometric coefficients. A mathematical description of the reaction schemes given by R.1 and R.2 captures the initial scorch delay and the curing for both isothermal and non-isothermal curing situations, but fails to account for reversion. In a subsequent publication, Ding et al. ${ }^{25}$ introduced an additional degradative side-reaction of the crosslinks to account for reversion; specifically,

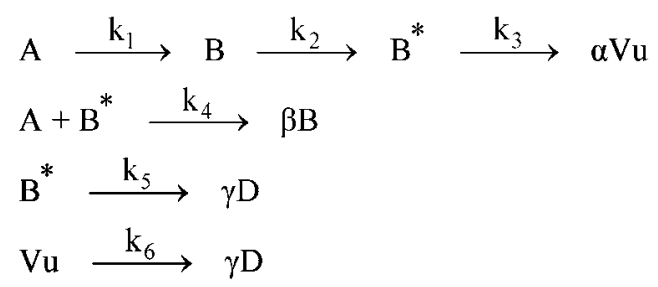

The kinetic models developed by Coran and coworkers ${ }^{19-25}$ describe some of the experimental ODR data and have significantly extended the state of the art; nevertheless, they have the following shortcomings:

1. The reaction scheme does not explicitly include sulfur; consequently, the models are unable to capture any dependence of the final vulcanizate on the initial concentration of sulfur.

2. The models do predict how final crosslink density depends on the initial accelerator concentration, but the dependence is not consistent with experiments. Specifically, the models predict that a reduction by one-half in the concentration of accelerator at constant sulfur concentration leads to a one-half reduction in the crosslink density, although experimentally the final crosslink density is only reduced by approximately a one-third.

3. The definitions of A, B and $\mathrm{B}^{*}$ are ambiguous because of the lumped nature of the model. Specifically, it is well known that the crosslinks and all precursor species are polysulfidic with a distribution of chain lengths. For example, assuming that $\mathrm{A}$ is the sum of all different lengths of the polysulfidic accelerator species (as would be the case in a lumped parameter formulation), it is not possible to close a detailed sulfur balance around the individual mechanistic steps.

4. In the proposed scheme, it is not obvious how to compute the concentration of A, (i.e. Acc- $\left.S_{x}-A c c\right)$ from the initial accelerator and sulfur concentrations, since there is no explicit kinetic equation specifying the production of A from initial concentrations of sulfur and accelerator. The initial concentration of A could be determined experimen tally, however this is a tedious experimental task and obviously not predictive. One 
could assume the formation of A to be extremely rapid such that the initial concentration of $\mathrm{A}$ is determined from the initial sulfur and accelerator concentrations depending upon which curative is stoichiometrically limiting. However, when the accelerator is the limiting curative, not all the sulfur would be consumed using this simple stoichiometric hypothesis.

5. The proposed reaction scheme does not take into account any co-products that may be formed at different stages of the process. For instance, the formation of B from A, will also involve the formation of a protonated co-product such as MBT, when MBS is used as the accelerator. Likewise, the reaction producing activated species $\mathrm{B} *$ from $\mathrm{B}$ will be accompanied by the formation of an accelerator-terminated polysulfidic species. These co-products could possibly have very significant roles to play in the actual vulcanization system.

6. Using the model parameters reported by Ding et al. ${ }^{24}$, more degradation product D is formed via the side reaction than crosslinks that are formed by the main vulcanization reaction. It is difficult to accept a kinetic scheme where the major product is from a side reaction.

In spite of the concerns raised above, the kinetic model of Coran is a significant advance that satisfies some of the basic features needed by a fundamental model and acknowledges some aspects of the underlying chemistry in a mechanistically reasonable manner.

An alternative kinetic analysis was developed by Chapman ${ }^{26}$ to explain the cure behavior of efficient sulfenamide systems. Isotopically-labeled accelerators and sulfur were used to obtain experimental insight into the nature of the intermediates formed, and a kinetic scheme involving the formation of pendant groups followed by reaction between two pendant groups to form crosslinks or cyclic structures was proposed. The basic mechanistic steps considered in this model were

$$
\begin{aligned}
& \mathrm{BtSNR}_{2}+\mathrm{S}_{8} \longrightarrow \mathrm{BtSS}_{\mathrm{x}} \mathrm{NR}_{2} \longrightarrow \mathrm{BtSS}_{\mathrm{x}} \bullet \cdot \mathrm{NR}_{2} \\
& \mathrm{BtSS}_{\mathrm{x}}+\text { Rubber } \longrightarrow \mathrm{BtSS}_{\mathrm{x}}-\text { Rubber } \\
& 2 \mathrm{BtSS}_{\mathrm{x}}-\text { Rubber } \longrightarrow \text { Rubber }-\mathrm{S}_{\mathrm{x}} \mathrm{S}_{\mathrm{x}}-\text { Rubber }+2 \mathrm{BtS} \bullet
\end{aligned}
$$

where $\mathrm{Bt}$ stands for the benzothiazole group and $\mathrm{BtSNR}_{2}$ is the accelerator 2-(morpholinothio) benzothiazole. However, mass action kinetic equations were not developed to describe the formation of pendant groups and sulfur crosslinks; rather, empirical equations guided by the experimental observation of the intermediates were developed. The equations derived by Chapman ${ }^{26}$ do predict the concentration of crosslinks in addition to some of the important vulcanization intermediates such as the pendant groups, but the generalization of these kinetic equations to other formulations is rather limited. Additionally, they fail to address some important reactions, specifically the formation of accelerator polysulfides and reversion chemistry.

While Coran and Chapman considered the kinetics of benzothiazolesulfenamide accelerated vulcanization systems, Duchacek ${ }^{27-29}$ performed kinetic studies to elucidate cure reactions in thiuram-accelerated vulcanization systems. For natural rubber systems accelerated with TMTD, a single first-order reaction was proposed; however, for cis-1,4-polybutadiene as the elastomer, the cure-curves were modeled as the sum of three independent first-order reactions: (i) fast crosslinking; (ii) slow crosslinking related to the formation of a ZDMC intermediate; and (iii) degradation with an induction period $t_{i}$. The fast-crosslinking reactions were assumed to be primarily ionic in nature, while the slow crosslinking and degradation reactions were assumed to be radical in nature. Although the models of Duchacek provide some insight into the reaction mechanisms of thiuram-accelerated systems, they fail to address the issue of how the concentration of 
different curatives affects the time evolution of crosslinks.

In our opinion a significant deficiency in all the kinetic models discussed above is the fact that the polysulfidic nature of the different reactants, intermediates and products is not explicitly acknowledged. Unless the lengths of each polysulfidic species are accounted for explicitly, it is not possible to close a sulfur mass balance around the individual reactions - a very serious shortcoming in any fundamental description. A very natural way to build in the information of polysulfidic nature of different species into the reaction equations is through population-balance models, the details of which will be discussed in Section IV of this paper. The concerns raised in the preceding paragraphs should not be interpreted as an overly harsh criticism of the models developed by previous researchers, since they do capture some significant features of accelerated vulcanization. Rather, these concerns illustrate the considerable complexity involved in developing a fundamental kinetic model of the vulcanization reactions.

\section{CHEMISTRY OF ACCELERATED SULFUR VULCANIZATION}

A fundamental kinetic model of vulcanization should acknowledge the mechanistic details of the underlying reactions. The objective of this Section is to provide a review of the reaction mechanisms that have been proposed to describe the chemistry of benzothiazolesulfenamide accelerated systems. We will not attempt a comprehensive discussion of the experimental methods that have been employed to study vulcanization chemistry, since this information has been discussed extensively in a number of excellent review articles ${ }^{2-7}$; rather, we will focus the review on a critical discussion of the reaction mechanisms that have been proposed for accelerated sulfur vulcanization. This Section will be organized around the general reaction scheme shown in Figure 1, where the chemistry is divided into three main processes. In Section III.A we will review the accelerator chemistry, focusing on the reactions between the accelerator, sulfur and the activator that lead to the formation of accelerator polysulfides. Section III.B, will be concerned with the reactions and intermediate chemical structures by which these accelerator polysulfides are converted into polysulfidic crosslinks, including the origin of the scorch delay reaction in sulfenamide accelerator systems. The post-crosslinking chemistry will be the focus of Section III.C, where we will review the reaction mechanisms that have been proposed for how the structure of the polysulfidic crosslinks evolves at longer cure times. Although the retarder chemistry, if present, is part of the accelerator chemistry, we will delay the discussion of reaction mechanisms associated with the presence of a retarder until Section III.D. Section III is lengthy; however, we believe that it is essential to critically review the rich chemistry of the sulfenamide accelerated systems prior to beginning the development in Section IV of the fundamental kinetic description using the population balance models.

\section{A. ACCELERATOR CHEMISTRY}

The first step in sulfur vulcanization is the formation of an active sulfurating species, which is a prerequisite for the formation of a crosslink precursor. A sulfurating species is a molecule that is able to insert sulfur in the form of crosslinks into the elastomer, where it has been long recognized that accelerator polysulfide complexes are better sulfurating species than molecular sulfur. These accelerator polysulfides are formed by the interaction of the accelerator molecule with molecular sulfur. ${ }^{2}, 20-23,30-32$ In the absence of an activator such as $\mathrm{ZnO}$, these polysulfides are of Type I:

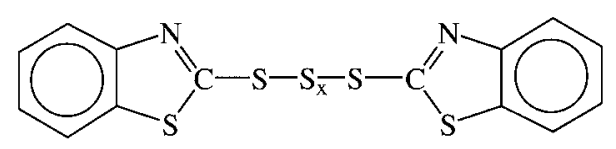


The organic pendant groups in the sulfurating species I is benzothiazole. For other accelerator systems as given in Table I, the organic pendant groups will be different, but the polysulfidic nature will be the same. When $\mathrm{ZnO}$ is present, the zinc complexes with the accelerator polysulfides, resulting in structures of Type II:

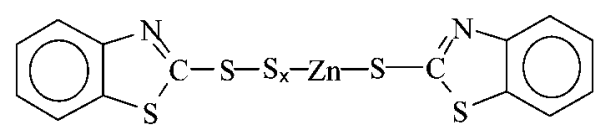

If ligands L such as amines and carboxylate ions are also present in the system, structures of Type III can result:

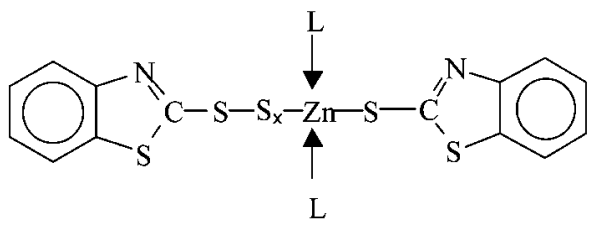

Structures of Type II and III seem to imply that zinc is covalently bonded as part of the polysulfidic chain in the accelerator species; however, it is more probable that the $\mathrm{Zn}$ complexes with the sulfur in the accelerator polysulfide as shown in IV, where the dotted lines indicate the formation of a complex of zinc with sulfur;

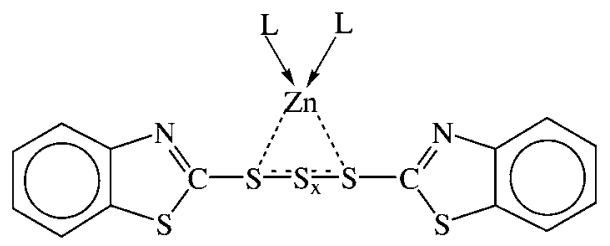

Structures of Type I, II and III or alternately IV are all capable of sulfurating the rubber chains and therefore are considered active sulfurating species.

In the remainder of this Section, we will consider the formation of structures of Type I from the reactions between the accelerator and sulfur. For benzothiazolesulfenamide accelerators, 2-bisbenzothiazole-2-2'-disulfide (MBTS) plays a major role, since MBTS reacts with sulfur to form 2-bisbenzothiazole-2-2'-polysulfides (MBTPs); and, MBTPs, or the zinc/amine complexes of MBTPs, are a key sulfurating intermediate. ${ }^{2,20-23,34-38}$ Since MBS is the accelerator of primary interest, we will discuss: (i) the formation of MBTS from MBS, which is the first step in the vulcanization process; followed by (ii) the mechanism for sulfur pick-up by MBTS to form MBTPs; and finally (iii) the exchange reactions that MBTPs can undergo. Finally, we will review the affect of $\mathrm{ZnO}$ on the formation of zinc-accelerator complexes of Type I, II and III. 
1. Formation of MBTS from MBS. - On heating MBS at vulcanization temperatures from 140 to $180{ }^{\circ} \mathrm{C}$, the S-N bond in the accelerator dissociates, liberating free amine and 2-mercaptobenzothiazole (MBT) as shown below for a generic benzothiazolesulfenamide.

$$
\mathrm{BtSNR}_{2} \longrightarrow \mathrm{BtSH}+\mathrm{R}_{2} \mathrm{NH}
$$

MBT can now react with the benzothiazolesulfenamide accelerator to form MBTS as follows: ${ }^{39-42}$

$$
\mathrm{BtSNR}_{2}+\mathrm{BtSH} \longrightarrow \mathrm{BtSSBt}+\mathrm{R}_{2} \mathrm{NH}
$$

For an MBS accelerated system, these two reactions are<smiles>c1ccc2sc(SN3CCOCC3)nc2c1</smiles>

and<smiles>CSc1nc2ccccc2s1</smiles>

R.7 and R.8 proceed via formation of an active intermediate, which has been postulated to be either ionic or radical in character. The argument for an ionic mechanism comes from the work of Son et al.., ${ }^{40}$ who investigated the reaction of MBT with TBBS, OBTS and CTP, where they proposed the following ionic transition-state:

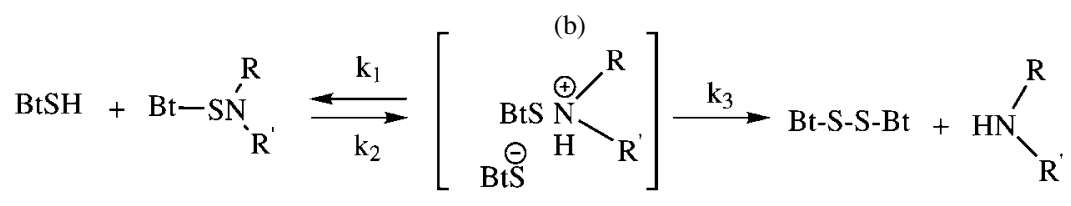

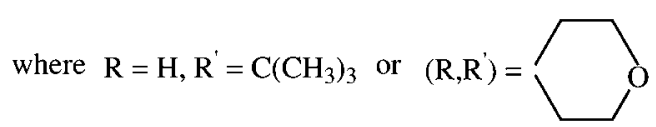

This postulated ionic transition state was based on the fact that R.9 leads to the rate-expressions

$$
\begin{aligned}
& -\frac{\mathrm{d}[\mathrm{Bt}-\mathrm{SH}]}{\mathrm{dt}}=\mathrm{k}_{1}[\mathrm{Bt}-\mathrm{SH}]\left[\mathrm{Bt}-\mathrm{SNR}_{2}\right]-\mathrm{k}_{2}[\mathrm{~b}] \\
& \frac{\mathrm{d}[\mathrm{Bt}-\mathrm{SS}-\mathrm{Bt}]}{\mathrm{dt}}=\mathrm{k}_{3}[\mathrm{~b}]
\end{aligned}
$$

which can quantitatively describe the experimental data for MBT depletion and MBTS formation. In Equation 1, $b$ denotes the transition state complex. Son et al. ${ }^{40}$ also considered an alter- 
native mechanism based on radical intermediates; specifically,

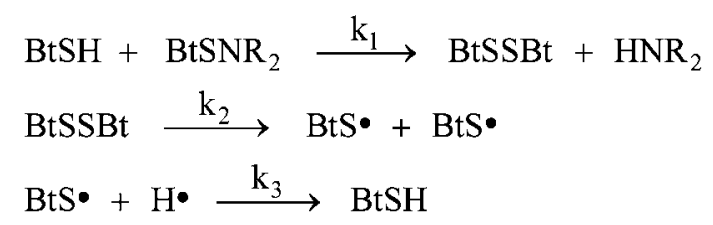

where $\mathrm{H}^{\bullet}$ comes from the solvent. The resulting kinetic equations are given by

$$
-\frac{\mathrm{d}[\mathrm{BtSH}]}{\mathrm{dt}}=\mathrm{k}_{1}[\mathrm{BtSH}]\left[\mathrm{BtSNR}_{2}\right]-\mathrm{k}_{2}\left[\mathrm{BtS}^{\bullet}\right]\left[\mathrm{H}^{\bullet}\right]
$$

and

$$
-\frac{\mathrm{d}\left[\mathrm{BtS}^{\bullet}\right]}{\mathrm{dt}}=\mathrm{k}_{3}\left[\mathrm{BtS}^{\bullet}\right]\left[\mathrm{H}^{\bullet}\right]-\mathrm{k}_{2}[\mathrm{BtSSBt}]=0
$$

where the standard steady state approximation is applied in order to equate Equation $2 b$ to zero. Combining Equations 2a and 2b,

$$
-\frac{\mathrm{d}[\mathrm{BtSH}]}{\mathrm{dt}}=\mathrm{k}_{1}[\mathrm{BtSH}]\left[\mathrm{BtSNR}_{2}\right]-\mathrm{k}_{2}[\mathrm{MBTS}]
$$

This kinetic expression for the radical mechanism can also describe the experimental data; however, Son et al. ${ }^{40}$ still conclude that the transition state is ionic in character. The argument against a radical mechanism is as follows: If the reaction proceeded by a radical intermediate, possibly the $\mathrm{BtS}^{\bullet}$ radical, one of the rate-limiting steps would involve the solvent donating an $\mathrm{H}^{\bullet}$ radical to the $\mathrm{BtS}^{\bullet}$ radical to form $\mathrm{MBT}$. This would imply that changing the solvent from an $\mathrm{H}^{\bullet}$ donor like toluene to a solvent incapable of donating $\mathrm{H}^{\bullet}$, like chlorobenzene, would dramatically change the rate of reaction of MBT; however, no change in the reaction rate was observed between toluene and chlorobenzene. Studies of reactions of related compounds also show significant enhancement in the reaction rate in polar solvents, which would also be consistent with an ionic transition state.

In contrast to the conclusions of Son et al., ${ }^{40}$ other researchers postulate that a radical mechanism controls the formation of MBTS from MBS. ${ }^{35-36,43-45}$ The free radical reactions are assumed to proceed by (i) S-N bond scission in MBS (generically represented as Bt-S-NR ${ }_{2}$ for an arbitrary amine) yielding $\mathrm{Bt}-\mathrm{S}^{\bullet}$ and $\mathrm{NR}_{2}$ radicals, (ii) $\mathrm{S}-\mathrm{H}$ bond scission in MBT resulting in Bt- $\mathrm{S}^{\bullet}$ and $\mathrm{H}^{\bullet}$ radicals, and (iii) subsequent recombination of two Bt-S radicals to form MBTS. This reaction mechanism is summarized below:

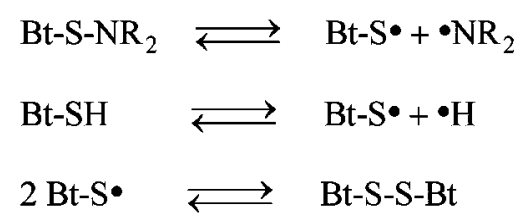

A variety of other exchange reactions can occur in addition to $\mathrm{R} .11$ as a result of reactions of MBS, MBT and MBTS with the Bt-S ${ }^{\bullet}$ radicals. In order to gain better insight into the interaction of the curatives during the early stages of vulcanization, Gradwell et al. ${ }^{35,36,45}$ studied the reactions of MBS, CBS, TBBS and MDB in the absence of polyisoprene both with and without sulfur in a DSC, where the products formed at 130 to $178^{\circ} \mathrm{C}$ were analyzed by HPLC. They found that on heating MBS in the absence of sulfur, MBT, or possibly an MBT-amine salt, and MBTS 
were the main products, which is consistent with R.11. In another study Gradwell et al. ${ }^{46}$ thermally decomposed MBTS, yielding MBTM, MBTPs and MBT as shown in Figure 4, where MBTM is the dominant product with small amounts of MBTP and MBT. The formation of MBTM and MBTP suggests exchange reactions between Bt- $\mathrm{S}^{\bullet}$, or more generally $\mathrm{Bt}-\mathrm{S}_{\mathrm{x}}{ }^{\bullet}$ radicals, with MBTS. For example, Bt-S $\mathbf{S}^{\bullet}$ radicals may undergo exchange reactions with MBTS to form $\mathrm{MBTM}$ and $\mathrm{Bt}-\mathrm{S}_{\mathrm{x}} \cdot$ radicals of higher sulfur rank as shown below: ${ }^{45-47}$

$$
\begin{gathered}
\mathrm{Bt}-\mathrm{S} \bullet+\mathrm{Bt}-\mathrm{S}-\mathrm{S}-\mathrm{Bt} \longrightarrow \mathrm{Bt}-\mathrm{S}-\mathrm{Bt}+\mathrm{Bt}-\mathrm{S}-\mathrm{S} \bullet \\
\mathrm{Bt}-\mathrm{S}-\mathrm{S} \bullet+\mathrm{Bt}-\mathrm{S}-\mathrm{S}-\mathrm{Bt} \longrightarrow \mathrm{Bt}-\mathrm{S}-\mathrm{Bt}+\mathrm{Bt}-\mathrm{S}-\mathrm{S}-\mathrm{S} \bullet
\end{gathered}
$$

The formation of MBTP can occur via the recombination of radicals; ${ }^{45-47}$ specifically, MBTS can form via R.14:

$$
\mathrm{Bt}-\mathrm{S} \bullet+\mathrm{Bt}-\mathrm{S} \bullet \quad \rightleftarrows \mathrm{Bt}-\mathrm{S}-\mathrm{S}-\mathrm{Bt}
$$

and although not explicitly proposed by Gradwell et al., ${ }^{45,46}$ consistency demands that the exchange reactions R.12 and R.13 and the recombination reaction R.14 be generalized to include longer length polysulfidic species which will yield MBTPs in addition to MBTM as shown below:

$$
\begin{aligned}
\mathrm{Bt}_{\mathrm{x}} \mathrm{S}_{\mathrm{x}}+\mathrm{Bt}-\mathrm{S}-\mathrm{S}-\mathrm{Bt} & \rightleftarrows \mathrm{Bt}-\mathrm{S}_{(\mathrm{x}+1)}-\mathrm{Bt}+\mathrm{Bt}-\mathrm{S}^{\bullet} \\
\mathrm{Bt}_{\mathrm{x}} \cdot+\mathrm{Bt}_{\mathrm{y}} \bullet & \longrightarrow \mathrm{Bt}-\mathrm{S}_{(\mathrm{x}+\mathrm{y})}-\mathrm{Bt}
\end{aligned}
$$

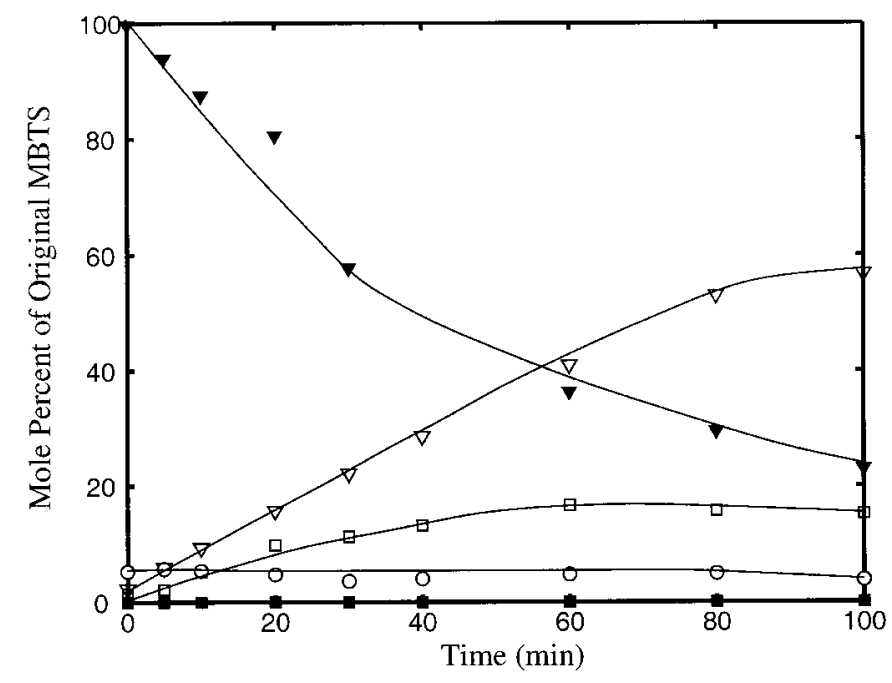

FIG. 4. - Products formed upon heating MBTS as a function of time at $178^{\circ} \mathrm{C} .(\boldsymbol{\nabla}) \mathrm{MBTS}$,

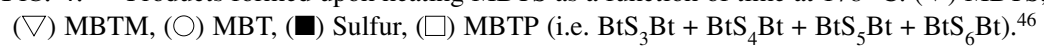
Adapted from M. H. S. Gradwell, B. Morgan, and W.J. McGill, "Rate of formation of polysulfides of 2-bidbenzothiazole-2-2'-disulfide in the presence of sulfur and 2-mercaptobenzothiazole,"

J. Appl. Polym. Sci. 56, 1581, Copyright @1995, with permission from John Wiley \& Sons. 
Reactions R.10 to R.16 summarize the important free radical reactions between the different accelerator species in the absence of sulfur. These reactions provide a mechanistic description of the formation of the key intermediate MBTS from MBS in addition to providing an explanation for MBTM and MBTP production. However an obvious concern is the stability of the free radical intermediates. Specifically, for these reactions to occur, the free radical intermediates must have sufficient stability so that they can diffuse to either BtSSBt or Bt- $\mathrm{S}_{\mathrm{x}}{ }^{\circ}$ and then react to form MBTM, MBTS or MBTP, without first reacting with the rubber. Since the diffusivity will decrease with increasing molecular size, the need for stabilization will become a more serious issue for the polysulfidic radicals with longer sulfur chains. Fairbrother et al. ${ }^{48}$ and Trivette et $a l .{ }^{49}$ have postulated increased stability of the higher molecular weight MBTP radicals due to resonance stabilization and formation of a three-electron bond between the two terminal sulfur atoms as shown below:

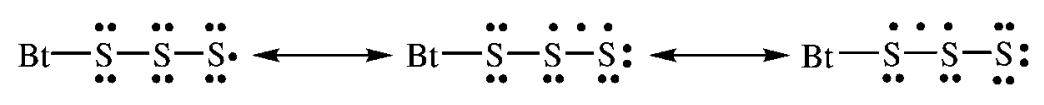

If $\mathrm{Bt}-\mathrm{S}_{\mathrm{x}} \cdot$ radicals are sufficiently stabilized, it may be possible to detect them experimentally. Although Bt-S ${ }_{x}{ }_{x}$ radicals were not directly observed, Gardner and Fraenke ${ }^{50}$ have observed ${ }^{\circ} \mathrm{S}$ $\mathrm{S}_{\mathrm{x}}-\mathrm{S}^{\bullet}$ diradicals using ESR. Additional indirect evidence for the existence of $\mathrm{Bt}-\mathrm{S}_{\mathrm{x}}{ }^{\bullet}$ radicals is provided in the ESR (or EPR) spectroscopic studies of Coleman et al. ${ }^{51,52}$ who studied the thermal decomposition of thiuram sulfides and their intermediates in accelerated sulfur vulcanization. These studies showed the existence of $\mathrm{Me}_{2} \mathrm{~N}(\mathrm{C}=\mathrm{S}) \mathrm{S}^{\bullet}$ radicals and persulfenyl radicals $\mathrm{Me}_{2} \mathrm{~N}(\mathrm{C}=\mathrm{S}) \mathrm{SS}_{\mathrm{x}}{ }^{\cdot}$ in accelerated sulfur vulcanizing systems. The thiuram persulfenyl radicals are resonance-stabilized by ring formation as shown below:<smiles>CNC(=S)SSCCNC1SSSS1</smiles>

Additional evidence for the existence of benzothiazole radicals comes from the work of Shelton and McDonel $^{53}$ who investigated the reaction mechanisms for sulfenamide accelerators using radical scavengers like benzoquinone, quinhydrone, hydroquinone, 2,5-di-tert-butylhydroquinone and 1,1-diphenylpoerylhydrazyl. They concluded that sulfur vulcanization in the presence of sulfenamide accelerators is mixed polar and free radical in nature, proceeding via radical accelerator intermediates. Manik and Banerjee ${ }^{54}$ studied the vulcanization of CBS accelerated natural rubber systems with and without the addition of dicumyl peroxide (DCP) to the formulation, to assess the presence of radical species in the vulcanization process. Specifically, since the peroxide radical is highly reactive, it would interfere with any radical reactions involving the accelerator complexes; in contrast, if the accelerator chemistry is primarily ionic, then there will be little affect upon the addition of DCP. They concluded that an NR/CBS/S system does proceed via radical intermediates; however, a mixed radical/polar mechanism results upon addition of zinc to the system.

In summary, several reaction schemes that lead to the formation of MBTS from MBS, as well as a series of exchange reactions that lead to MBTPs have been proposed. The reaction of MBTS with elemental sulfur, as will be discussed subsequently can also produce MBTPs; thus, the presence of MBTPs as shown in Figure 4 does not necessarily require the occurrence of exchange reactions R.12, R.13 and R.15. Reaction R.11 assumes that the formation of MBTS from MBS proceeds via a radical intermediate, while R.9 assumes the transition state to have an 
ionic character. The question of whether the transition state is ionic or radical in nature is interesting; however, from the perspective of developing a kinetic description, which is our eventual objective, it is not critical, since the rate equations for the depletion of MBS and MBT and the formation of MBTS are the same for both mechanisms.

2. Reaction of Sulfur with MBTS and Other Accelerator-Polysulfides. - For MBS accelerated vulcanization, a key intermediate that reacts with elemental sulfur is MBTS, where MBTS cleaves homolytically on heating to produce $\mathrm{BtS}{ }^{\bullet}$ radicals as shown below. ${ }^{4,52}$

$$
\mathrm{Bt}-\mathrm{S}-\mathrm{S}-\mathrm{Bt} \rightleftarrows \mathrm{Bt}-\mathrm{S} \bullet+\mathrm{Bt}-\mathrm{S} \bullet
$$

Although the unsymmetrical cleavage as shown below is also possible, it is not as likely, since it requires breaking of the more stable C-S bond.

$$
\mathrm{Bt}-\mathrm{S}-\mathrm{S}-\mathrm{Bt} \quad \mathrm{Bt} \bullet+\mathrm{Bt}-\mathrm{S}-\mathrm{S} \bullet
$$

The Bt-S ${ }^{\bullet}$ radical may also undergo reaction with elemental sulfur to form longer length radicals; specifically.

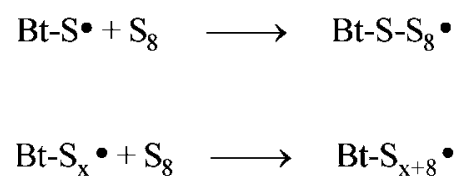

The $\mathrm{Bt}-\mathrm{S}_{\mathrm{x}+8} \cdot$ radicals can then combine with each other via $\mathrm{R} .16$ or undergo exchange reaction with MBTS via R.15 to form accelerator polysulfides. Although Bt- $\mathrm{S}_{\mathrm{x}+8}$ radicals have not been directly observed, they would be consistent with a ring opening free radical addition of molecular sulfur. Implicit in the R.19 mechanisms are the assumptions that (i) molecular sulfur is primarily present as an eight member ring and (ii) the sulfur pick-up reaction totally consumes $\mathrm{S}_{8}$ rather than incorporating just part of the $S_{8}$ ring. In the vulcanization literature, reactions similar to R.19 are often proposed ${ }^{2,52,53,55-58}$ where a lumped description (i.e. where x can be 1,2 , etc) is used, thus, bypassing the mechanistic details as well as not explicitly closing the mass balance on sulfur. Gradwell et al. ${ }^{46}$ experimentally measured the distribution of products resulting from the reactions of MBTS and subsequently formed MBTPs with sulfur as shown in Figure 5. Compared with the data in Figure 4 where MBTS is heated in the absence of sulfur, the addition of sulfur enhances the production of MBTPs, while diminishing the production of MBTM. This would imply that reactions of Bt-S radicals with $\mathrm{S}_{8}$ via R.19 take place in preference to exchange reactions with MBTS (i.e. R.12, R.13 and R.15), which is not surprising considering the relatively high concentration of sulfur compared to other species that need to participate in the exchange reactions. 


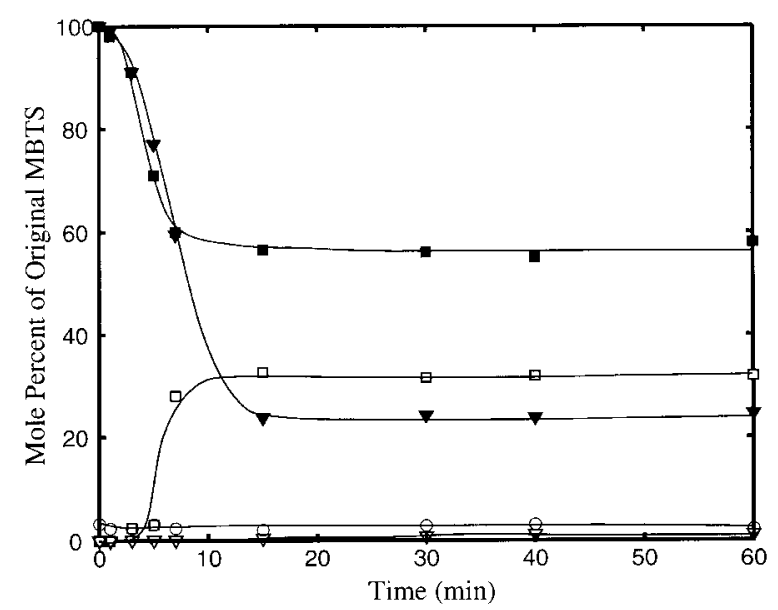

FIG. 5. - Products formed from the reaction of MBTS and sulfur in a 1:1 molar ratio at $150{ }^{\circ} \mathrm{C}$. $(\boldsymbol{\nabla})$ MBTS, $(\nabla)$ MBTM, (O) MBT, ( $\square$ ) Sulfur, $(\square)$ MBTP (i.e. $\mathrm{BtS}_{3} \mathrm{Bt}+\mathrm{BtS}_{4} \mathrm{Bt}+\mathrm{BtS}_{5} \mathrm{Bt}+\mathrm{BtS}_{6} \mathrm{Bt}$ ). Adapted from M. H. S. Gradwell, B. Morgan, and W. J. McGill, "Rate of formation of polysulfides of 2-bidbenzothiazole-2-2'-disulfide in the presence of sulfur and 2-mercaptobenzothiazole," J. Appl. Polym. Sci. 56, 1581, Copyright @1995, with permission from John Wiley \& Sons.

The diffusional behavior of $\mathrm{Bt}_{-} \mathrm{S}^{\bullet}$, or in general the $\mathrm{Bt}-\mathrm{S}_{\mathrm{x}}{ }^{\bullet}$ radicals, is important both in the initial stages of the vulcanization, where the sulfur-accelerator complex is being formed, as well as later during the crosslinking chemistry (see Section III.B), where Bt- $\mathrm{S}_{\mathrm{x}} \cdot{ }$ radicals react with the allylic hydrogens to form crosslink precursors. Since the concentration of allylic hydrogens in the isoprene chains is higher than the concentration of any other reactive species, it seems a rather low probability event that two $\mathrm{Bt}-\mathrm{S}_{\mathrm{x}}{ }^{\cdot}$ radicals, which are only present in small concentrations, would (i) diffuse together to form an accelerator polysulfide as required in R.16 without reacting first with an allylic hydrogen in the elastomer backbone, which are present in large concentrations, or (ii) react with sulfur, which is also present in relatively high concentrations during the initial stages of the vulcanization. Thus, it is problematic to explain the formation of longer length MBTPs like Bt-S - -Bt by R.12 through R.16, since only small concentrations of the most abundant accelerator species MBTS is present. However, consider the scenario where the Bt-S-S-Bt reacts in a concerted manner with sulfur as shown below:

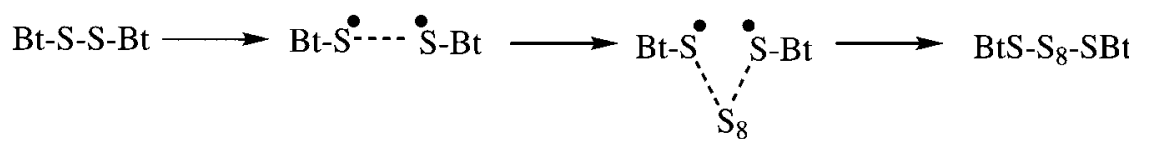

Bt-S-S-Bt may fragment into two Bt-S ${ }^{\bullet}$ radicals, which do not have sufficient time to diffuse apart due to the high viscosity of the rubber matrix. Because of the close proximity of the pair of $\mathrm{BtS}^{\bullet}$ radicals and the presence of a high concentration of sulfur during the initial stages of the vulcanization, it is more probable that the pair of $\mathrm{BtS}^{\bullet}$ radicals initially pick-up sulfur and form species like BtS-S - SBt. However, during the later stages of the cure when most of the sulfur has been consumed, $\mathrm{Bt}-\mathrm{S}_{\mathrm{x}}{ }^{\cdot}$ radicals will have the opportunity to diffuse further away from each other and may then react with the allylic hydrogens in the isoprene chain. In R.20 there is no need to even postulate the formation of distinct free radicals, only that Bt-S-S-Bt and sulfur react in a concerted manner where the transition state is not explicitly specified. A concerted reaction scheme like that of R.20 is consistent with the previously discussed reactions as well as addresses the concern that allylic hydrogens are present in relatively high concentrations. The sulfur 
pick-up reactions, suppressing the explicit indication of free radical intermediates, can be written as:

$$
\begin{array}{ll}
\mathrm{Bt}-\mathrm{S}-\mathrm{S}-\mathrm{Bt}+\mathrm{S}_{8} & \longrightarrow \\
\mathrm{Bt}-\mathrm{S}_{\mathrm{x}}-\mathrm{Bt}+\mathrm{S}_{8} & \longrightarrow \mathrm{Bt}_{8}-\mathrm{S}_{\mathrm{x}+8}-\mathrm{Bt}-\mathrm{Bt}
\end{array}
$$

In addition to $\mathrm{Bt}-\mathrm{S}_{\mathrm{x}}{ }^{\circ}$, MBTS and MBTP picking-up elemental sulfur to form accelerator-polysulfides, it has been proposed that MBT reacts with sulfur to form MBT-polysulfides; ${ }^{22,23,43,57,58}$ specifically,

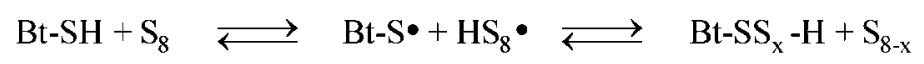

However, Gradwell et al. ${ }^{46}$ in their study of sulfur vulcanization of polyisoprene with benzothiazole accelerators, report no direct experimental evidence for the formation of $\mathrm{BtS}_{\mathrm{x}} \mathrm{H}$ from the interaction of MBT and sulfur, concluding that should such a species form, it will have very limited stability.

In Reactions R.19 through R.22, it is implied that the whole $S_{8}$ molecule is incorporated into the accelerator complex in a single step. However, an alternative mechanism for sulfur activation is indicated by the experimental results of Geyser and $\mathrm{McGill}^{59}$ on the formation of thiuram polysulfides (TMTPs) from the reaction of TMTD and sulfur and the studies of Gradwell et al. ${ }^{46}$ on the formation of MBTPs via the reaction of MBTS and sulfur. The experimental results of Geyser and McGill ${ }^{59}$ are shown in Figure 6, where TMTPs with lower sulfur length are produced in higher concentrations than TMTPs with higher sulfur lengths. Similar trends in the production of MBTPs of different polysulfidic lengths were also observed by Gradwell et al. ${ }^{45} \mathrm{~A}$ careful examination of the data in Figure 6 indicates that early in the reaction (i.e. times less than a minute) short chain length TMTPs are formed prior to the TMTPs with longer chain lengths. Similar trends are also observed in the formation of MBTPs..$^{45}$ This implies that it is unlikely that sulfur is inserted as $\mathrm{S}_{8}$ in TMTD or MBTS via R.21. Specifically, if this were the case then TMTPs and MBTPs with sulfur lengths of eight or greater would be produced initially followed by the desulfuration of these longer lengths through rearrangement reactions (see R.27 in the next Section) to produce the corresponding shorter lengths, which does not agree with the data. Thus, the data seems to indicate that the insertion of sulfur is sequential, i.e. one or two sulfur atoms at a time. If one assumes that a free radical mechanism is operative, then the operative reactions are:

$$
\begin{gathered}
\mathrm{Bt}-\mathrm{S} \bullet+\mathrm{S}_{8} \longrightarrow \mathrm{Bt}-\mathrm{S}-\mathrm{S} \bullet+\mathrm{S}_{7} \\
\mathrm{Bt}-\mathrm{S}-\mathrm{S} \bullet+\mathrm{S}_{\mathrm{x}} \longrightarrow \mathrm{Bt}-\mathrm{S}-\mathrm{S}-\mathrm{S} \bullet+\mathrm{S}_{\mathrm{x}-1}
\end{gathered}
$$

The lifetime of $\mathrm{BtS}^{\bullet}$ radical in the presence of high concentrations of allylic hydrogens still remains a concern for the proposed mechanism of sequential sulfur insertion; thus, instead of R.23, it may be more appropriate to describe MBTP formation by a reaction scheme that proceeds through a concerted mechanism analogous to R.20; specifically,

$$
\mathrm{Bt}-\mathrm{S}-\mathrm{S}-\mathrm{Bt}+\mathrm{S}_{\mathrm{x}} \longrightarrow \mathrm{Bt}-\mathrm{S}-\mathrm{S}-\mathrm{S}-\mathrm{Bt}+\mathrm{S}_{\mathrm{x}-1}
$$




$$
\text { Bt-S-S }-\mathrm{S}-\mathrm{Bt}+\mathrm{S}_{\mathrm{x}} \longrightarrow \mathrm{Bt}-\mathrm{S}-\mathrm{S}_{\mathrm{y}+\mathrm{l}}-\mathrm{S}-\mathrm{Bt}+\mathrm{S}_{\mathrm{x}-1}
$$

The data from Geyser and McGill ${ }^{59}$ shown in Figure 6 and Gradwell et al. ${ }^{46}$ provide strong evidence for R.23 or R.24. However, R.21 cannot be eliminated in favor of R.24 by only examining the concentration profiles of the accelerator-polysulfides of lengths less than eight, since similar trends could still be explained by sulfur insertion as $S_{8}$ followed by the desulfuration of higher sulfur lengths that are greater than or equal to eight via the exchange reactions to be discussed in the next Section. For example, consider BtSSBt picking-up sulfur as $\mathrm{S}_{8}$ molecule to form $\mathrm{BtSS}_{8} \mathrm{SBt}$, which subsequently undergoes an exchange reaction with BtSSBt to yield BtSSSBt and $\mathrm{BtSS}_{7} \mathrm{SBt}$. In this case, BtSSSBt will be formed prior to $\mathrm{BtSS}_{2} \mathrm{SBt}$ or $\mathrm{BtSS}_{3} \mathrm{SBt}$ and so on. One could eliminate reaction R.21 in favor of R.23 or R.24 if direct measurement of the $S_{x}$ species for $\mathrm{x}=1$ to 8 were available, since the reactions R.23 and R.24 require the existence of such species at finite concentrations. Unfortunately, such measurements, to the best of our knowledge, have not been reported. However, Morgan and McGill ${ }^{60}$ have recently measured the concentration of long length sulfurating species formed on heating MBTS and sulfur in the presence of $\mathrm{ZnO}$. The data for MBTP formation are shown in Figure 7, where initially the smaller length accelerator-polysulfides are formed with subsequent production of longer lengths. Significantly, the trend continues for $\mathrm{BtSS}_{8} \mathrm{SBt}$ and higher length MBTPs. The lower length accelerator-polysulfides are formed prior to longer length accelerator-polysulfides for polysulfidic species with sulfur lengths both less than and greater than 8 , which strongly suggests that sulfur insertion is sequential. It must be noted that the data shown in Figure 7 is for formulations that contain $\mathrm{ZnO}$; however, in our opinion the presence of $\mathrm{ZnO}$ should merely increase the rate of reactions but not affect the basic trends in the product distribution. In light of this, we believe that sulfur insertion is most probably sequential, proceeding via Reaction R.24.

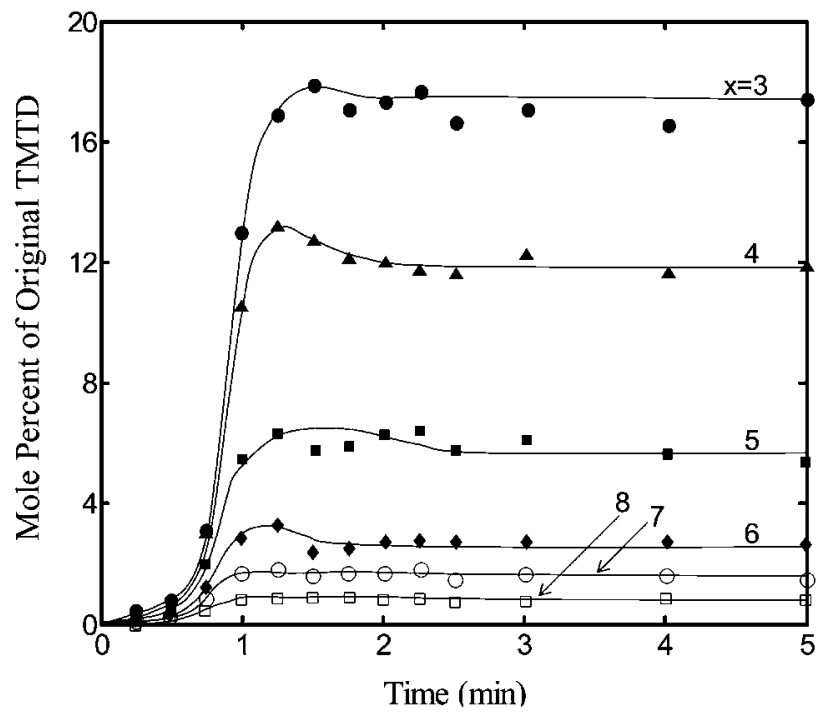

FIG. 6. - Formation of thiuram polysulfides from the reaction of TMTD and sulfur in a 1:0.25 molar ratio at $130{ }^{\circ} \mathrm{C} . \mathrm{X}=3$ indicates $\left(\left(\mathrm{CH}_{3}\right)_{2} \mathrm{NC}=\mathrm{SS}_{3} \mathrm{~S}=\mathrm{CN}\left(\mathrm{CH}_{3}\right)_{2}\right.$ and so on. Adapted from M. Geyser and W.J. McGill, "A study of the rate of formation of polysulfides of tetramethylthiuram disulfide," J. Appl. Polym. Sci. 55, 215, Copyright $@ 1995$, with permission from John Wiley \& Sons. 


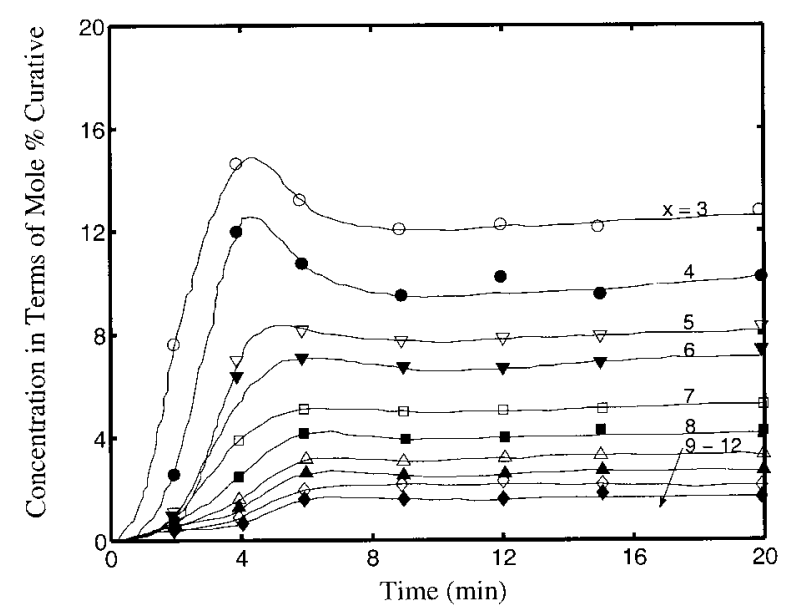

FIG. 7. - HPLC analysis of the formation of MBTP upon heating MBTS, sulfur and ZnO in a 1:1:1 molar ratio isothermally at $152^{\circ} \mathrm{C} . \mathrm{X}=3$ indicates $\mathrm{BtS} \mathrm{S}_{3} \mathrm{Bt}$ and so on.

A key question concerning reactions R.23 and R.24 is the precise nature of the species $S_{x}$. Specifically, is $S_{x}$ a stable ring structure like $S_{8}$ or is $S_{x}$ a biradical polymeric sulfur? Muller ${ }^{61}$ and Semlyen ${ }^{62}$ have characterized sulfur rings extensively along with their bond angles, bond lengths, torsional angles and conformations and concluded that among the different $\mathrm{S}_{\mathrm{x}}$ species, only $\mathrm{S}_{8}, \mathrm{~S}_{7}$ and $\mathrm{S}_{6}$ exist as stable rings. The species $\mathrm{S}_{6}$ is cyclic, highly symmetric and has normal bond distances of $2.08 \mathrm{~A}$, bond angles of $105 \pm 3^{\circ}$ and torsional angles of $85 \pm 15^{\circ}$, the $\mathrm{S}_{7}$ ring has longer bond lengths of 2.04 to $2.18 \mathrm{~A}$, bond angles of 101.9 to $107.4^{\circ}$ and torsional angles of 0.4 to $108.8^{\circ}$, while $S_{8}$ has a simple crown structure of puckered eight-membered ring with bond lengths of $2.04 \mathrm{~A}$, bond angles of $105 \pm 3^{\circ}$ and torsional angles of $98 \pm 10^{\circ}$. These stable ring structures can exist only at temperatures below $160{ }^{\circ} \mathrm{C},{ }^{63}$ which is within the range of cure temperatures of most vulcanization systems. This would imply that it should be possible to detect at least the species $\mathrm{S}_{6}$ and $\mathrm{S}_{7}$, besides $\mathrm{S}_{8}$ in the vulcanization mixture; however, to the best of our knowledge these cyclic species have not been directly observed in vulcanization systems. Alternatively, $\mathrm{S}_{\mathrm{x}}$ species could be biradical sulfur species and the existence of such biradicals in liquid sulfur at temperatures above $172{ }^{\circ} \mathrm{C}$ has been reported by Gardner ${ }^{50}$ and Koningsberger ${ }^{64}$, 65 using ESR spectroscopy. Here again we are not aware of any ESR studies of vulcanization systems where biradical species were observed. Until such studies are carried out, it is not possible to precisely specify the nature of the $S_{x}$ species.

3. Exchange Reaction Among Accelerator Polysulfides. - MBTPs that are formed by the insertion of sulfur into MBTS can also undergo exchange reactions among themselves. These exchange reactions are important, since they affect the eventual length distribution of accelerator polysulfides, which in turn controls the length distribution of crosslinks. Clear evidence for the existence of such exchange reactions is provided by the experimental studies of $\mathrm{R}-\mathrm{S}_{\mathrm{x}}-\mathrm{R}$ compounds with radioactively labeled sulfur, where $\mathrm{R}$ is an alkyl or aryl group. ${ }^{67,68,69}$ Specifically, the studies involved exchange reactions between ${ }^{35} \mathrm{~S}$-labeled disulfide with trisulfides and of ${ }^{35} \mathrm{~S}$ labeled trisulfides with tetrasulfides, which are summarized below where the fraction of radioactively labeled compound is indicated by $1 *$ or $1 / 2^{*}$ or $1 / 3^{*}$.

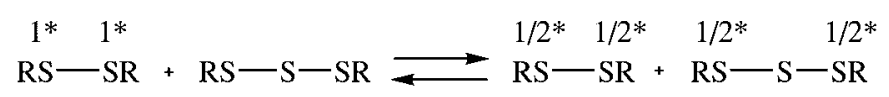




$$
\mathrm{RS}-\mathrm{S}-\mathrm{SR}+\mathrm{RS}-\mathrm{S}-\mathrm{S}-\mathrm{SR} \rightleftarrows \mathrm{RS}-\stackrel{1 / 3^{*}}{\rightleftarrows}-\mathrm{SR}+\mathrm{RS}-\mathrm{1/3^{* }}-\mathrm{S}-\mathrm{S}-\mathrm{SR}
$$

The C-S bond in R-S $-\mathrm{R}$ is unreactive, but the $\mathrm{S}-\mathrm{S}$ bonds are quite labile, taking part in sulfur-exchange reactions. Since $\mathrm{Bt}-\mathrm{S}_{\mathrm{x}}-\mathrm{Bt}$ are accelerator polysulfides of the type $\mathrm{R}-\mathrm{S}_{\mathrm{x}}-\mathrm{R}$ and the sulfur bonds in the interior of $\mathrm{Bt}-\mathrm{S}_{\mathrm{x}}-\mathrm{Bt}$ have similar lability, exchange reactions similar to R.25 and R.26 should be anticipated for MBTPs. Specifically,

$$
B t-S_{x}-B t+B t-S_{y}-B t \quad \longrightarrow \quad B t-S_{z}-B t+B t-S_{x+y-z}-B t
$$

This reaction is similar to the exchange reactions R.12, R.13 and R.15 discussed previously for the formation of MBTPs from $\mathrm{BtS}_{\mathrm{x}}{ }^{\cdot}$ and MBTS. R.27 is written without any explicit designation of free radical intermediates and could just as well proceed via a concerted mechanism like that given in R.20 for MBTS and $\mathrm{S}_{8}$.

4. Effect of $\mathrm{ZnO}$ - Formation of Zinc-Accelerator Complexes. - When zinc or equivalently zinc oxide is present as an activator in the vulcanization system, it catalyzes the formation of macromolecular complexes of types (II), (III) or (IV). These macromolecular complexes can be either internally chelated, as in dimethyl dithiocarbamate (VII) or may form a benzothiazolezinc-complex like (VIII). ${ }^{2,3,5,10-12,69}$

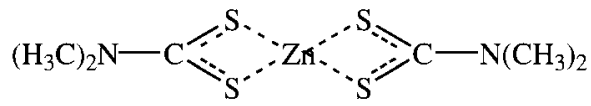

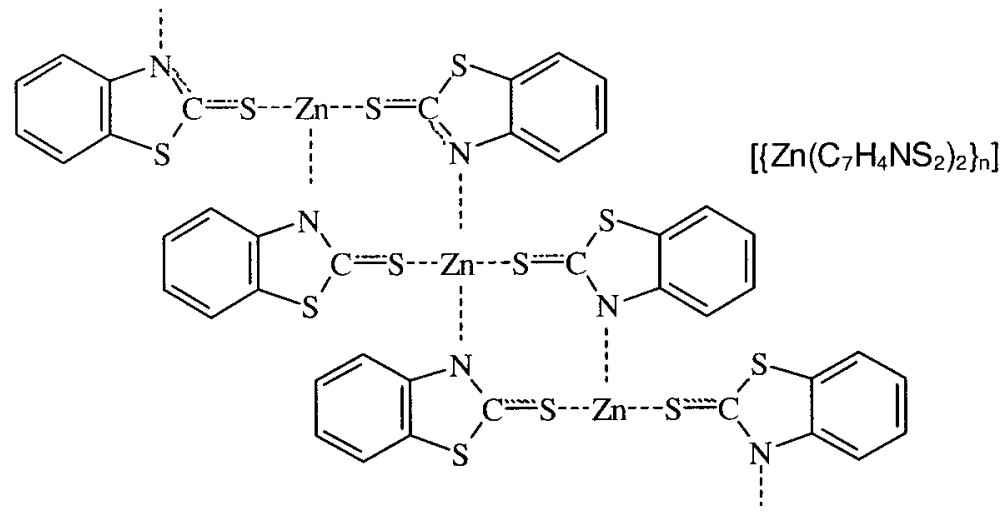

None of these structures have appreciable solubility in rubber or significant reactivity towards sulfur. However, the solubility and reactivity are enhanced if the zinc coordinates with an amine that is released from the sulfenamide or with carboxylate ligands, which are present in systems with stearic acid. A typical structure of the zinc/accelerator ligand system is shown below: 


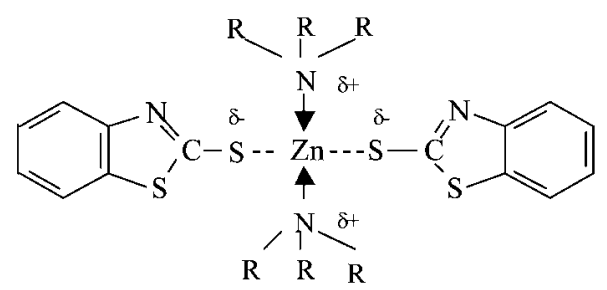

The effect of $\mathrm{ZnO}$ on the vulcanization process has been studied for a variety of accelerators. $^{20,32,36-38,70-72}$ These studies have revealed that $\mathrm{ZnO}$ readily forms zinc-complexes with MBT upon heating; specifically,

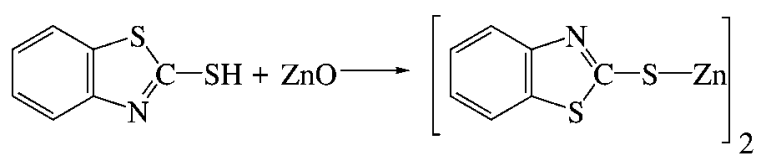

However, a similar reaction is not observed upon heating $\mathrm{ZnO}$ with MBTS. ${ }^{60,71-73}$

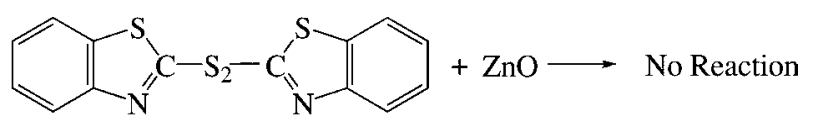

Although the reaction of $\mathrm{ZnO}$ with MBT readily occurs, it does not proceed to completion. ${ }^{32,37,74}$ The incomplete reaction is most probably due to the formation of an insoluble layer of ZMBT on the surface of $\mathrm{ZnO}$ particles, which prevents further reaction of $\mathrm{ZnO}$ with MBT.

Campbell and Wise ${ }^{22,23}$ studied MBT, MBTS and MBS accelerated vulcanization of natural rubber both in the presence and absence of zinc oxide and stearic acid. The amount of extractable zinc decreased as a function of time, which indicates the formation of complexes of type BtS$\mathrm{Zn}_{\mathrm{S}} \mathrm{x}_{\mathrm{x}} \mathrm{SBt}$. However, it was not conclusively determined whether BtS-Zn-S $-\mathrm{SBt}$ was the only sulfurating structure produced, since it is possible that BtS-S $-\mathrm{SBt}$ species were formed concurrently. Gradwell and $\mathrm{McGill}^{36}$ investigated the interaction of sulfenamide accelerators with sulfur, $\mathrm{ZnO}$ and stearic acid in the absence of rubber and found that $\mathrm{ZnO}$ did not react with any of the sulfenamide accelerators like MBS, CBS or TBBS. This implies that the only way to form complexes of Type III in sulfenamide accelerated systems is if MBT reacts with $\mathrm{ZnO}$ to form ZMBT, which could eventually form BtS-Zn-S - -SBt. As discussed previously with regard to R.7, MBT is initially formed by the thermal decomposition of MBS; however, the MBT is quickly converted to MBTS via reaction R.8. Consequently, it is likely that only very small amounts of zinc-accelerator complexes BtS-Zn-S $-\mathrm{SBt}$ are formed at the early stages of the reaction and zinc free structures BtS-S $-\mathrm{SBt}$ dominate. During subsequent crosslinking reactions, additional MBT is formed (see R.34), and it is probable that the majority of BtS-Zn-S $-\mathrm{SBt}$ structures be formed during this later stage of the vulcanization process.

Like their zinc-free counterparts, BtS-Zn-S $-\mathrm{SBt}$ species are also active sulfurating species. However, unlike BtS-S $-\mathrm{SBt}$, which inserts sulfur via a radical mechanism, the sulfur pick-up reaction in BtS-Zn-S $-\mathrm{SBt}$ appears to be ionic or polar in nature. Krebs ${ }^{75}$ proposed that the zincaccelerator complexes activate sulfur in an analogous fashion as the activation by amines, via the ionic cleavage of sulfur 


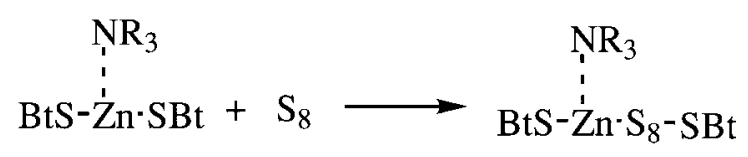

In contrast, Bateman et al. ${ }^{2}$ postulate ionic-cleavage, where one of the sulfur atoms in the zincaccelerator complex causes a nucleophilic attack on the $\mathrm{S}_{8}$ ring; specifically,

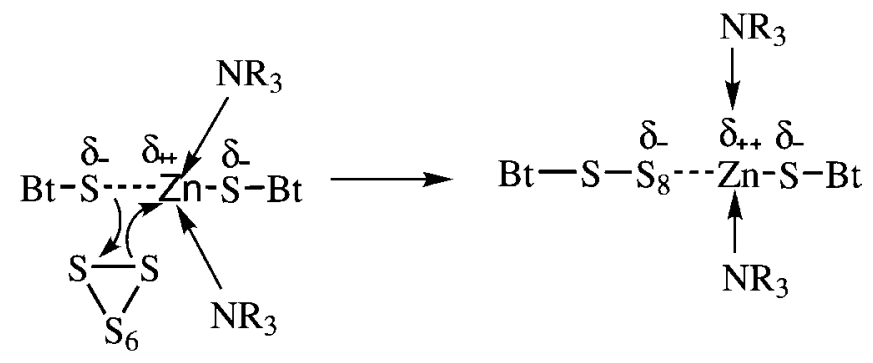

Although R.30 and R.31 imply that sulfur activation occurs via $\mathrm{S}_{8}$ ring incorporation, the arguments in favor of sequential sulfur activation as discussed previously for the zinc-free structures BtS-S $-\mathrm{xBt}$ may still be appropriate for these zinc-accelerator complexes. In a manner similar to R.24 for zinc-free systems, zinc-accelerator complexes may also undergo exchange reactions with other zinc-accelerator complexes to yield a distribution of $\mathrm{Zn}$-accelerator species; that is,

$$
\mathrm{Bt}-\mathrm{Zn}-\mathrm{S}_{\mathrm{x}}-\mathrm{Bt}+\mathrm{Bt}-\mathrm{Zn}-\mathrm{S}_{\mathrm{y}}-\mathrm{Bt} \quad \longrightarrow \quad \mathrm{Bt}-\mathrm{S}_{\mathrm{z}}-\mathrm{Zn}-\mathrm{Bt}+\mathrm{Bt}-\mathrm{S}_{\mathrm{x}+\mathrm{y}-\mathrm{z}}-\mathrm{Zn}-\mathrm{Bt}
$$

The formation of both zinc-free and zinc-accelerator complexes are critical steps in the accelerated-sulfur vulcanization of elastomers. While it is clear that $\mathrm{BtS}-\mathrm{S}_{\mathrm{x}}-\mathrm{SBt}$ is the active sulfurating agent in the absence of $\mathrm{ZnO}$, both BtS-S $-\mathrm{SBt}$ and BtS-Zn- $\mathrm{S}_{\mathrm{x}}-\mathrm{SBt}$ may be active sulfurating agents in the presence of $\mathrm{ZnO}$. Indirect evidence for such a proposition also comes from the nature of the vulcanization mechanism; specifically, whether the process is polar or radical. Manik and Banerjee ${ }^{76}$ studied the vulcanization of natural rubber with CBS in the presence and absence of $\mathrm{ZnO}$ and concluded that the $\mathrm{CBS} /$ zinc/stearic acid system reacted initially by a radical mechanism; however, during the later stages of the cure, the main crosslinking reactions were polar in nature. In contrast, the reaction was completely free radical in nature in the absence of $\mathrm{ZnO}$ and stearic acid. Similar observations were made by Shelton and $\mathrm{McDonel}^{53}$ and Morita and Young ${ }^{77}$ who concluded that sulfenamide-sulfur systems are mixed radical/polar in the presence of $\mathrm{ZnO}$. Since BtS-S $-\mathrm{SBt}$ reacts via radical chemistry and $\mathrm{BtS}-\mathrm{Zn}-\mathrm{S}_{\mathrm{x}}-\mathrm{SBt}$ via a polar mechanism, this might explain why one observes a mixed radical and polar chemistry in sulfenamide accelerated systems.

\section{B. CROSSLINKING CHEMISTRY}

The crosslink formation reactions are initiated by the accelerator polysulfides $\mathrm{BtS}-\mathrm{S}_{\mathrm{x}}-\mathrm{SBt}$ and BtS-Zn-S $-\mathrm{SBt}$ that are formed by the mechanisms discussed in the previous Section. Crosslinks are formed via crosslink precursors, which are rubber bound intermediates. In this Section we will first present the experimental evidence for the existence of crosslink precursors and their role in sulfur vulcanization, followed by a discussion on the mechanisms by which they are formed. The chemistry in this Section will be divided into the following three sub-Sections: 
Formation of Crosslink Precursors, Conversion of Crosslink Precursors to Crosslinks and Scorch Delay.

1. Formation of Crosslink Precursors. - Crosslink precursors are formed when the accelerator polysulfides react with the rubber chains, resulting in structures $\mathrm{RS}_{\mathrm{x}}-\mathrm{SBt}$ that consist of an accelerator terminated polysulfidic pendant group attached to a rubber molecule (R). The existence of such crosslink precursors was first proposed by Scheele and Frank ${ }^{78}$ in their investigation of the NR/ZnO/TMTD/Sulfur system. They observed that the increase in bound sulfur exhibited the same first-order rate behavior as the reduction of TMTD concentration until a maximum in bound sulfur was reached. Moore and Watson ${ }^{79}$ confirmed the presence of rubber-bound precursors by showing that an undercured vulcanizate, where all extra-network compounds apart from $\mathrm{ZnO}$ and $\mathrm{ZnS}$ had been extracted, could undergo additional crosslinking with an additional loss of nitrogen and sulfur. Further evidence of polythiobenzothiazole groups attached to natural rubber was obtained by Campbell and Wise ${ }^{22,23}$ in their study of NR-vulcanization accelerated by MBT. The development of ${ }^{13} \mathrm{C}$-NMR has made it possible to observe crosslink intermediates in precise detail. ${ }^{80}$ Using ${ }^{13} \mathrm{C}$-NMR, Koenig and coworkers ${ }^{81-83}$ were able to not only show the existence of crosslink precursors, but also distinguish between different precursors depending on the position of sulfur attachments in the rubber. Skinner's ${ }^{84}$ study of CBS-accelerated vulcanization using model compounds revealed that the sulfur attachments to the rubber hydrocarbon occur almost exclusively at the allylic positions, although this need not be the case in unaccelerated vulcanization systems. ${ }^{85}$ Skinner's work suggests that the presence of an accelerator enables an efficient substitution in allylic position, which agrees with the conclusions of Bateman et al. ${ }^{2}$, Lautenschlaeger ${ }^{86-88}$ and Kresja and Koenig. ${ }^{34,81}$

In the 1960s a radical mechanism that did not include crosslink precursors was advocated for the conversion of accelerator polysulfides into crosslinks; ${ }^{89-93}$ specifically,

$$
\begin{array}{ll}
\mathrm{BtSS}_{\mathrm{x}} \mathrm{SBt} & \rightleftarrows \mathrm{BtSS}_{\mathrm{y}} \bullet+\mathrm{BtSS}_{\mathrm{z}} \bullet(\mathrm{x}=\mathrm{y}+\mathrm{z}) \\
\mathrm{BtSS}_{\mathrm{y}} \bullet+\mathrm{RH} \longrightarrow \mathrm{R} \bullet+\mathrm{BtSS}_{\mathrm{y}} \mathrm{H} \\
\mathrm{R} \bullet+\mathrm{R} \bullet \longrightarrow \mathrm{R}-\mathrm{R} \\
\mathrm{R} \bullet+\mathrm{S}_{8} \longrightarrow \mathrm{R}_{8} \bullet \longrightarrow \mathrm{R}_{8}-\mathrm{R}
\end{array}
$$

where $\mathrm{R}$ is the rubber and $\mathrm{R}^{\bullet}$ is a free radical on the rubber chain. The reaction scheme given in R.33a was modified by Coleman et al. ${ }^{52}$ to include the formation of crosslink precursors as well as exchange reactions and radical recombination. The proposed reaction scheme is given by

$$
\begin{array}{ll}
\mathrm{BtSS}_{\mathrm{x}} \mathrm{SBt} & \longleftrightarrow \mathrm{BtSS}_{\mathrm{y}} \bullet+\mathrm{BtSS}_{\mathrm{z}} \bullet(\mathrm{x}=\mathrm{y}+\mathrm{z}) \\
\mathrm{BtSS}_{\mathrm{y}} \bullet+\mathrm{RH} & \mathrm{R} \bullet+\mathrm{BtSS}_{\mathrm{y}} \mathrm{H} \\
\mathrm{R} \bullet+\mathrm{BtSS}_{\mathrm{x}} \mathrm{SBt} & \longrightarrow \mathrm{RS}_{\mathrm{y}} \mathrm{SBt}+\mathrm{BtSS}_{\mathrm{x}-\mathrm{y}} \bullet \\
\mathrm{R} \bullet+\mathrm{BtSS}_{\mathrm{x}} \bullet & \mathrm{RSS}_{\mathrm{x}} \mathrm{Bt}
\end{array}
$$

The rationale behind the radical mechanism stemmed from the observation of accelerator polysulfidic radicals, although the proposed alkenyl radicals $\mathrm{R}^{\bullet}$ were never observed. The proposed formation of the alkenyl radical would require hydrogen abstraction by the accelerator polysulfidic radicals from rubber, which seems unlikely because (i) accelerator polysulfidic radicals are resonance-stabilized (i.e. Structures V and VI) and are therefore more stable than the alkenyl rad- 
icals; and (ii) the consequence of such hydrogen abstraction leads to species like $\mathrm{BtSS}_{\mathrm{y}} \mathrm{H}$, which are thought to have very limited stability. ${ }^{46}$

Coran ${ }^{94,95}$ proposed a non-radical, concerted mechanism for crosslink-precursor formation. He identified that the common feature of most of the accelerators is that they all contained a proton-acceptor site. For benzothiazole accelerators, this site is the nitrogen atom in the benzothiazole moiety. The proposed concerted mechanism is shown below, where the precursor is formed via an eight-membered transition state and MBT is the by-product of this reaction. ${ }^{20,21,34,95}$

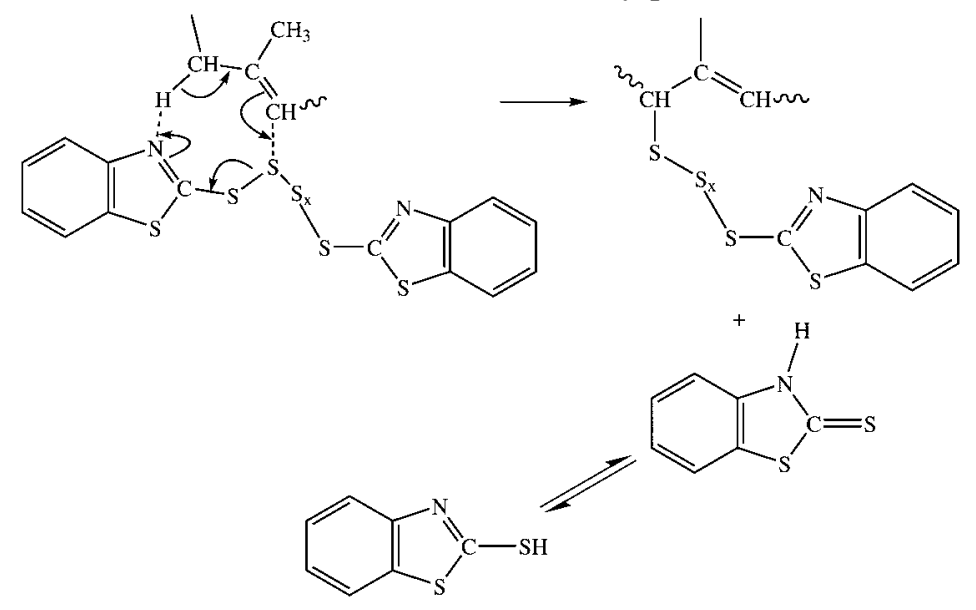

Gradwell et al., ${ }^{33}$ however question the formation of MBT at this step of the reaction, since for polyisoprene vulcanization accelerated with MBS and MDB, they did not observe any MBT formation prior to crosslinking. They propose that in the case of MBS accelerated systems, polysulfides of MBS are formed which are then added to the chain as benzothiazole pendant groups liberating free amine as shown below; first

$$
\mathrm{Bt}-\mathrm{S}-\mathrm{NR}_{2}+\mathrm{S}_{8} \longrightarrow \mathrm{Bt}-\mathrm{S}_{\mathrm{x}}-\mathrm{NR}_{2}
$$

and then<smiles>[R10]N[R16]</smiles>

R.36 depends upon R.35; however, as discussed earlier Bt-S-NR 2 readily converts to BtS-SBt via R.5 and R.6 in preference to sulfur activation to form Bt- $\mathrm{S}_{\mathrm{x}}-\mathrm{NR}_{2}^{8}$ as required in R.35. Thus there may be some potential problems with the scheme given in R.35 and R. 36 .

If zinc is complexed to the accelerator-polysulfide, the mechanism of crosslink-precursor formation has been traditionally explained by a polar mechanism ${ }^{2}$ as shown in R.37. However, the mechanism might be better described as a concerted mechanism involving a six-membered aromatic ring in the transition state, ${ }^{3}$ where $\mathrm{ZnS}$ is formed as a product in this reaction. 


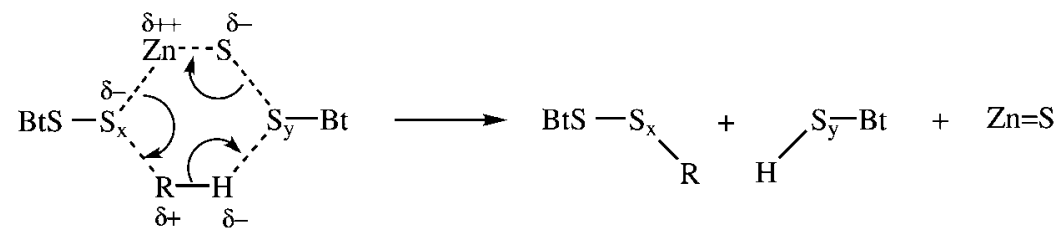

The rate of the sulfuration is governed by the relative energetics of formation of the new bond

$$
\mathrm{BtS}-\mathrm{S}_{\overline{\mathrm{X}}}^{-\mathrm{A}}-\mathrm{R}-\mathrm{R}
$$

versus the breaking of the old bond,

$$
\overbrace{\mathrm{H}-\mathrm{I}}^{\delta+}-\mathrm{S}_{\mathrm{y}}-\mathrm{Bt}
$$

The presence of zinc in the sulfurating complex provides a nucleophilic character to the sulfur attached to the zinc in the polysulfide as indicated below:

$$
\begin{array}{cc}
\delta+\stackrel{\delta}{\delta} & \delta-\delta+ \\
\mathrm{BtS}_{\mathrm{x}}-\mathrm{S}-\mathrm{Zn}-\mathrm{S}-\mathrm{S}_{\mathrm{y}} \mathrm{Bt}
\end{array}
$$

The presence of amines further increases the nucleophilicity of the sulfur in the polysulfidic complex, thereby increasing the rate of precursor formation. The persulfenyl species BtS- $\mathrm{S}_{\mathrm{x}}{ }^{\delta-}$ attacks an allylic site in the rubber via an $\mathrm{S}_{\mathrm{N}}{ }^{2}$ mechanism whereas the species $\mathrm{Bt}-\mathrm{S}_{\mathrm{y}}{ }^{\mathrm{d}}{ }^{2}$ is terminated with a hydrogen atom forming $\mathrm{Bt}-\mathrm{S}_{\mathrm{y}} \mathrm{H}$, and zinc is liberated as $\mathrm{ZnS}$. In R.37 the location of the split in the zinc-accelerator complex depends upon the position on the sulfur chain where zinc is complexed, implying that species like $\mathrm{Bt}-\mathrm{S}_{\mathrm{y}} \mathrm{H}$ can be formed. However for $\mathrm{y}$ greater than 1 , the species Bt-S $\mathrm{y}$ are thought to be unstable ${ }^{46}$ and only MBT (i.e. BtSH) is stable. This implies that either (i) the bond scission always takes place at the S-S bond next to the benzothiazole moiety resulting in MBT or (ii) that $\mathrm{Bt}-\mathrm{S}_{\mathrm{y}} \mathrm{H}$ is formed, which is rapidly transformed to some other species. The first situation would be plausible if zinc primarily complexes with the nitrogen atom on the benzothiazole moiety as indicated below:

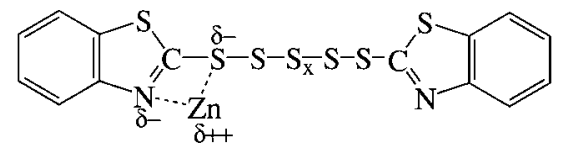

However if zinc complexes are formed with any two sulfur atoms on the polysulfidic chain, Bt$\mathrm{S}_{\mathrm{y}} \mathrm{H}$ would be a reaction product, which could undergo rapid reactions like sulfur pick-up, attachment to allylic sites on the rubber chain and recombination with another $\mathrm{Bt}-\mathrm{S}_{\mathrm{x}} \mathrm{H}$ species to give an accelerator polysulfide.

In summary, the formation of the crosslink-precursor is a key stage in accelerated sulfur vulcanization. The crosslink precursors can be formed from both BtS- $\mathrm{S}_{\mathrm{x}}-\mathrm{SBt}$ and BtS-Zn-S $-\mathrm{SBt}$ species. While both radical and concerted mechanisms have been proposed for forming crosslink precursors from BtS-S $-\mathrm{SBt}$, Coran's concerted mechanism seems more probable. In the case of zinc-accelerator complexes, the mechanism proposed by Bateman and coworkers ${ }^{2}$ is still generally accepted. The mechanism does answer some of the fundamental questions including formation of zinc-sulfide and role of amines, although the exact nature and position where zinc complexes in the accelerator complex remains unknown, which could have important bearing on the reaction mechanism and the product distribution. 
2. Conversion of Crosslink Precursors to Crosslinks. - Crosslink precursors are highly reactive, which makes direct observation of their reaction mechanisms difficult. Various mechanisms have been proposed for the conversion of the crosslink precursors into crosslinks. Bateman et $a l .{ }^{2}$ and Dogadkin et al. ${ }^{96}$ have suggested that crosslinks are formed upon disproportionation of two precursor moieties involving interchange of S-S bonds catalyzed by $\mathrm{BtS}^{-}$or similar persulfenyl anions; specifically,

$$
\begin{aligned}
& \mathrm{RS}_{\mathrm{x}} \mathrm{SBt}+\mathrm{BtS}^{-} \longrightarrow \mathrm{RS}_{\mathrm{z}}^{-}+\mathrm{BtS}_{(\mathrm{x}+2-\mathrm{z})} \mathrm{Bt} \\
& \mathrm{RS}_{\mathrm{z}}^{-}+\mathrm{RS}_{\mathrm{x}} \mathrm{SBt} \longrightarrow \mathrm{RS}_{2 \mathrm{z}} \mathrm{R}+\mathrm{BtS}_{(\mathrm{x}+1-\mathrm{z})}^{-}
\end{aligned}
$$

Subsequently, it was proposed that crosslinks were formed through reactions involving an exchange of rubber-bound pendant groups with zinc-accelerator complexes followed by a reaction with a rubber molecule as shown below: ${ }^{2}$

$$
\mathrm{RS}_{\mathrm{x}} \mathrm{SBt}+\mathrm{BtSS}_{\mathrm{x}} \mathrm{ZnS}_{\mathrm{y}} \mathrm{SBt} \longrightarrow \mathrm{RS}_{\mathrm{x}} \mathrm{ZnS}_{\mathrm{x}} \mathrm{SBt}+\mathrm{BtSS}_{\mathrm{y}} \mathrm{SBt}
$$

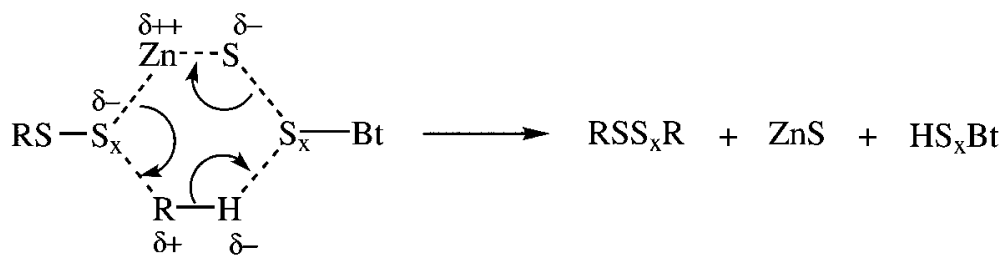

The difference between R.38 and R.39 as compared to R.40 and R.41 is that R.38 and R.39 involve the reaction between two precursor molecules, while R.40 and R.41 involve the reaction between a precursor molecule and the rubber. Both these reactions are catalyzed by the presence of zinc-accelerator complexes; however, these reactions can occur even in the absence of zinc, but the reaction rates are significantly lower. ${ }^{97}$

The disproportionation reaction can explain the formation of crosslinks of different lengths; however, it cannot account for the formation of monosulfidic crosslinks. Thus, if disproportionation is the primary mechanism for crosslink formation, monosulfidic crosslinks would have to come from the desulfuration reactions in the post-crosslinking chemistry (see Section III.C.1). Another possible objection to a disproportionation scheme stems from consideration of the diffusional mobility; specifically, the disproportionation requires two crosslink precursors to diffuse next to each other, which is unlikely because these are bulky molecules (i.e. isoprene chains with an attached accelerator moiety) with limited mobility. The recent work of Morgan and McGill ${ }^{98}$ on model-compound reaction studies of 2,3-dimethyl-2-butene (TME) accelerated with MBTS provides an additional objection to the disproportionation mechanism. On heating the disulfide crosslink precursor TME-S-SBt in n-hexane, crosslinked products were not detected, products which should have been formed if crosslinking occurred via a precursor-precursor interaction. These objections indicate that precursor-precursor interaction is probably not the primary mechanism of crosslink formation.

The formation of crosslinks by the direct reaction of the crosslink precursors with the rubber chain has been proposed by Coran ${ }^{20,99}$ and is given below: 


$$
\begin{aligned}
& \mathrm{RS}_{\mathrm{x}} \mathrm{SBt} \longrightarrow \mathrm{RS}_{\mathrm{y}} \bullet+\mathrm{BtS}_{\mathrm{z}} \bullet \\
& \mathrm{RS}_{\mathrm{y}} \bullet+\text { Rubber } \longrightarrow \mathrm{RS}_{\mathrm{y}} \mathrm{R}
\end{aligned}
$$

The reaction is assumed to proceed by a radical mechanism where the crosslink-precursor splits into two active radicals: (i) a persulfenyl radical $\mathrm{RS}_{\mathrm{y}}{ }^{\cdot}$ which can react with an allylic carbon on a neighboring isoprene chain and thus form a crosslink, and (ii) a benzothiazole terminated polysulfidic radical, $\mathrm{BtS}_{\mathrm{z}}{ }^{\circ}$, which can undergo a variety of reactions like radical recombination to yield $\mathrm{BtSS}_{\mathrm{x}} \mathrm{SBt}$ (i.e. R.14 and R.16), sulfur activation to form higher ranked radicals (i.e. R.19 and R.23) and addition to an isoprene chain to form another crosslink precursor. As written, R.42 allows for the breakage of any sulfur-sulfur bond in the crosslink precursor but Coran et al..$^{20,21}$ suggest that the weakest bond in the precursor molecule is the sulfur-sulfur bond adjacent to the benzothiazole group. Thus, the dominant reaction is most likely:

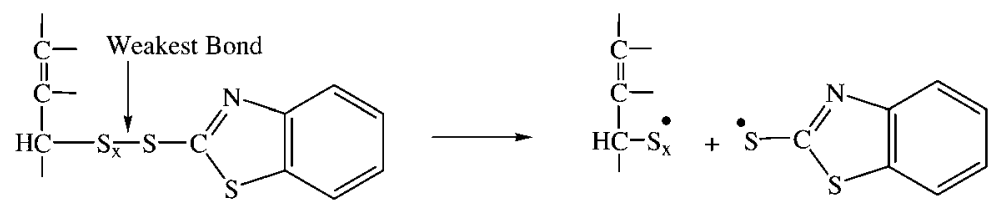

Recent quantum chemical calculations carried out in our group indicate that the strength of the $\mathrm{S}-\mathrm{S}$ bond decreases with the distance from the end organic group, where the decrease is rapid initially after which it levels off. Thus bond strength calculations argue against R.44 being the dominant $\mathrm{B} \rightarrow \mathrm{B}^{*}$ reaction. In the presence of $\mathrm{Zn}^{2+}$ it has been suggested that zinc complexes with the benzothiazole moiety attached to the rubber molecule: ${ }^{99}$

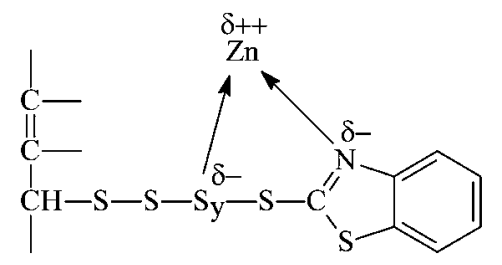

(XIV)

The zinc chelation can stabilize other sulfur bonds and may thereby change which bond is most likely to break. Thus, in the presence of zinc many of the S-S bonds can break; specifically,

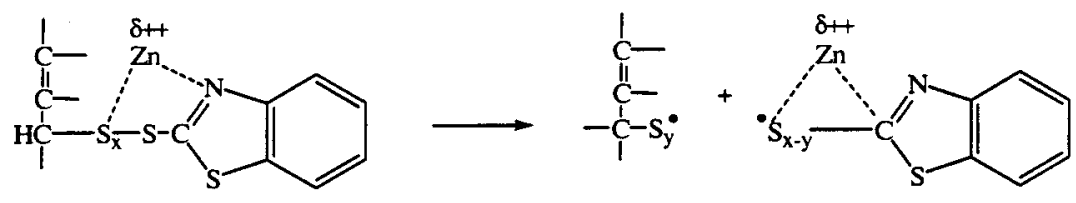

Kruger and McGill ${ }^{100}$ have also argued that a radical mechanism controls the formation of crosslinks from crosslink-precursors in the absence of zinc, where the reactions proceed via homolytic splitting of S-S bonds in the precursor molecule. Specifically, 


$$
\begin{aligned}
& \text { RS-S }-\mathrm{SBt} \quad \rightleftarrows \quad \mathrm{RS}_{\mathrm{x}} \mathrm{S}_{\mathrm{x}} \bullet+\mathrm{BtS} \bullet \\
& \mathrm{RS}-\mathrm{S}_{\mathrm{x}} \bullet+\mathrm{R}^{\prime} \mathrm{S}-\mathrm{S}_{\mathrm{y}}-\mathrm{Bt} \longrightarrow \mathrm{RS}_{\mathrm{x}}-\mathrm{S}_{\mathrm{y}}-\mathrm{R}^{\prime}+\mathrm{BtS} \bullet
\end{aligned}
$$

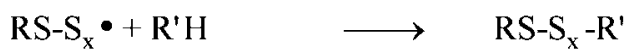

Unlike Coran ${ }^{20,94}$ who advocates the reaction of the persulfenyl radical with a neighboring rubber chain to be the reaction responsible for crosslink formation, Kruger and McGill ${ }^{100}$ propose that an additional pathway to crosslink formation may exist through the reaction of persulfenyl radicals with a crosslink precursor. This additional pathway given in R.47a is analogous to the pathway given earlier in R.39, the only difference being that R.39 assumes an ionic intermediate as opposed to a radical intermediate assumed by Kruger and McGill. ${ }^{100}$ Even though this additional pathway may be chemically plausible, the limited diffusional mobility of two bulky species like $\mathrm{R}-\mathrm{S}_{\mathrm{x}}-\mathrm{S}^{\bullet}$ and $\mathrm{R}-\mathrm{S}-\mathrm{S}_{\mathrm{x}}-\mathrm{SBt}$ argue that such a reaction would be unlikely. Another possibility for crosslink formation may involve reaction between two $\mathrm{RS}_{\mathrm{x}} \cdot$ radicals analogous to that postulated for the accelerator radical in R.14 and R.16. Specifically,

$$
\begin{aligned}
& \mathrm{RS}_{\mathrm{x}} \bullet+\mathrm{RS}_{\mathrm{y}} \bullet \\
& \mathrm{RS}_{\mathrm{x}} \bullet+\mathrm{RS}_{\mathrm{y}} \mathrm{Bt}
\end{aligned}
$$

Although this reaction may be chemically feasible, here again this reaction pathway would most probably be a minor contribution to the crosslinking, because of the limited mobility of these large molecules

Considering R.42 through R.48, it is clear that the conversion of precursors to crosslinks proceeds via generation of two different radicals - a persulfenyl radical $\mathrm{R}-\mathrm{S}_{\mathrm{y}} \cdot$ and a benzothiazole terminated polysulfidic radical $\mathrm{Bt}_{-} \mathrm{S}_{\mathrm{x}} \cdot$. While the conversion of persulfenyl radicals to crosslinks is their major reaction, alternate reactions are possible for this radical intermediate. The same is true for the $\mathrm{Bt}-\mathrm{S}_{\mathrm{x}}$ radical since these two radicals are identical except for the terminal organic groups and consequently would be expected to undergo similar reactions. This implies that if R.43 occurs for the $\mathrm{R}_{-} \mathrm{S}_{\mathrm{y}}{ }^{\prime}$ species, a similar reaction should also occur for the Bt$\mathrm{S}_{\mathrm{x}}$ ' species, where the polysulfidic benzothiazole radical would be added to the rubber chain resulting in a crosslink precursor. Specifically,

$$
\mathrm{BtS}_{\mathrm{x}} \bullet+\text { Rubber } \longrightarrow \text { Rubber-S } \mathrm{x}-\mathrm{Bt}
$$

If $\mathrm{x}$ is equal to 1 in the above reaction, an inactive crosslink precursor would be formed due to the more stable C-S bond that would have to be broken to form the active persulfenyl radical. Likewise, since $\mathrm{Bt}_{-} \mathrm{S}_{\mathrm{y}}{ }^{*}$ is capable of reacting with sulfur to form polysulfides of higher sulfur rank via R.19 and R.23, the persulfenyl radical $\mathrm{R}_{-\mathrm{S}} \mathrm{x}_{\mathrm{x}}$ would be expected to undergo a similar reaction as given below:

$$
\text { Rubber- } \mathrm{S}_{\mathrm{x}} \bullet+\mathrm{S}_{8} \longrightarrow \text { Rubber- } \mathrm{S}_{\mathrm{x}+8} \bullet
$$




$$
\text { Rubber- } \mathrm{S}_{\mathrm{x}} \bullet+\mathrm{S}_{8} \longrightarrow \text { Rubber- } \mathrm{S}_{\mathrm{x}+1} \bullet+\mathrm{S}_{7}
$$

In addition, chemical consistency requires the formation of cyclic sulfides. In R.43 the persulfenyl radical $\mathrm{R}-\mathrm{S}_{\mathrm{y}}{ }^{\cdot}$ reacted with an allylic carbon in a neighboring rubber chain to form a crosslink. However, it is also possible for the radical to react with an allylic carbon in the backbone chain from which it originates and thereby form a loop as shown below:

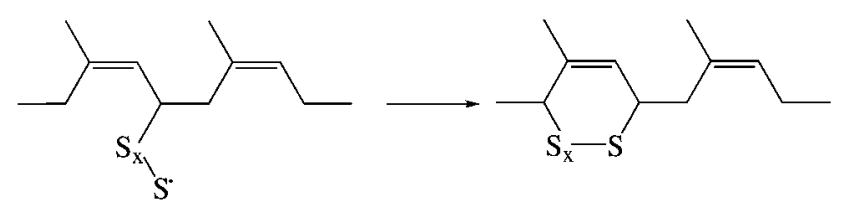

The resulting cyclic sulfide will be elastically ineffective, and the formation of these elastically ineffective crosslinks will be discussed in more detail in Section V.

In conclusion, crosslink formation most likely proceeds through the reaction of the crosslink precursor with an allylic carbon in the neighboring rubber chain via a persulfenyl radical intermediate; however, the disproportionation reaction still remains a possibility. The persulfenyl intermediate and the associated accelerator terminated polysulfidic radical must undergo similar reactions, since they are chemically similar species except for the terminal group whose effect will become negligible as the length of the polysulfidic radical increases. Finally, chemical consistency requires the formation of cyclic sulfides, where the persulfenyl radical can react with an allylic carbon on the parent chain just like it can react with allylic carbons on the neighboring chain.

3. Scorch Delay Reactions. - Premature vulcanization during processing is known as scorch and is generally undesirable. One of the technological advantages of benzothiazole sulfenamide accelerators is the delay at the onset of vulcanization, which is called 'Scorch Delay'. Two different explanations have been proposed to account for scorch delay: (i) thermal stability of the accelerator and (ii) exchange reactions between accelerator-derived intermediates.

Bateman $e t$ al. $^{2}$ proposed that scorch delay in benzothiazole sulfenamides results because these accelerators only begin to exert their full accelerative action when the vulcanization temperature is reached. The crosslinking reactions cannot start unless there is formation of the polysulfidic sulfurating species, which depends on the formation of MBTS. However, MBTS cannot be formed unless MBT reacts with MBS via R.6 and R.8; hence, if the release of MBT from MBS via R.7 is delayed, there is an overall delay in the crosslinking reaction. Since MBT is formed from MBS by the thermal decomposition of the S-N bond, the onset of vulcanization is delayed pending decomposition of the S-N bond. Scheele and coworkers ${ }^{101}$ employ similar arguments suggesting that the amine salt of MBT or persulfenyl MBT is the first product of sulfenamides, which then leads to the activation of elemental sulfur. The stability of these sulfenamides towards heat, hydrolysis, and/or reduction determines the length of the induction period and, hence, the scorch delay.

Exchange reactions between various accelerator-derived intermediates have also been proposed to explain the observed delay. Coran ${ }^{20}$ proposed that the delay observed in sulfenamide accelerators is due to persulfenyl radical quenching by the accelerator polysulfides BtS-S $-\mathrm{SBt}$ and/or BtS-Zn-S - -SBt. Specifically, the polymeric persulfenyl radicals R-S ${ }_{\mathrm{y}}$ react with rubber to form crosslinks via R.43 and if the persulfenyl radicals are rapidly quenched prior to forming crosslinks, crosslink formation would be significantly impeded as shown below: 


$$
\mathrm{BtS}-\mathrm{S}_{\mathrm{x}}-\mathrm{SBt}+\mathrm{R}-\mathrm{S}_{\mathrm{y}} \bullet \longrightarrow \mathrm{R}_{\mathrm{z}}-\mathrm{SBt}+\mathrm{BtS}-\mathrm{S}_{\mathrm{x}+\mathrm{y}-\mathrm{z}} \bullet
$$

R.53 would dominate until most of the accelerator polysulfides are consumed after which R-S ${ }_{\mathrm{y}}$ could form crosslinks. Increasing the accelerator concentration would increase the efficiency of R.53 thereby increasing the scorch delay.

Gradwell and McGill ${ }^{102}$ proposed a different exchange reaction as the origin of the scorch delay. In their study of isoprene rubber vulcanization by CBS accelerator, the scorch delay was attributed to exchange reactions between the accelerator and $\mathrm{Bt}-\mathrm{S}_{\mathrm{x}}$ terminated pendant groups to form amine-terminated pendant groups (step 3 in R.54). The amine-terminated pendant groups may subsequently participate in a variety of reactions to eventually form crosslinks; however, their reactivity is lower than the $\mathrm{BtS}-\mathrm{S}_{\mathrm{x}}$ terminated pendant groups and hence the delay. Specifically,

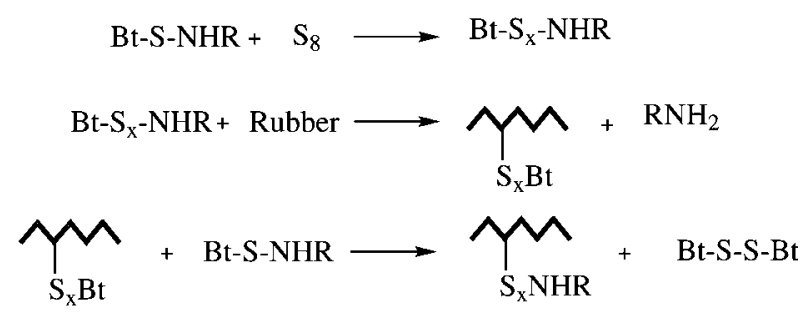

where $\mathrm{BtS}_{\mathrm{x}} \mathrm{NHR}$ is the sulfenamide accelerator (i.e. for CBS, $\mathrm{R}$ is $\mathrm{C}_{6} \mathrm{H}_{11}$ ). Thus CBS initially reacts with sulfur to form $\mathrm{CBPs}$, which because of their high reactivity rapidly react with the rubber chain. A substitution reaction leads to polysulfidic benzothiazole terminated pendant groups, where cyclohexylamine is liberated. The benzothiazole-terminated pendant groups subsequently undergo an exchange reaction with the accelerator, forming an amine-terminated pendant group liberating MBTS. The amine-terminated pendant groups have a lower reactivity; thus, the rate of conversion of these groups into crosslinks is much slower than the rate of conversion of the benzothiazole-terminated pendant groups and, hence, the scorch delay. Although the first step in R.54 suggests direct sulfur activation by CBS to form CBPs, such a reaction might not be the dominant reaction when CBS is heated, because, analogous to MBS, CBS can be readily converted to MBTS (i.e. via R.5 and R.6). Even if CBPs are not formed and MBTS is formed instead, the exchange reaction given in R.54 could still be effective, since MBTS would activate sulfur producing MBTPs via R.21 and R.24, which would then yield benzothiazole-terminated pendant groups as required for the scorch delay reaction in the third reaction in R.54.

The basic premise of the scorch delay via R.54 is the slower conversion of amine-terminated pendant groups to crosslinks as compared to benzothiazole-terminated pendant groups. However, the exact details of the reactions leading to the conversion of amine-terminated pendant groups into crosslinks are still not fully understood. Gradwell and McGill ${ }^{102}$ suggest that these pendant groups might be involved in an exchange reaction with benzothiazole-terminated pendant groups to form crosslinks as shown below:

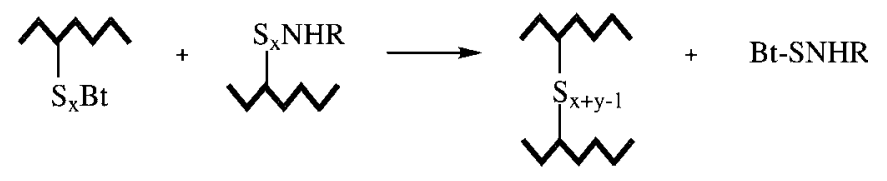

However, even if such a reaction is chemically plausible, it is unlikely to be a dominant reaction 
for crosslink formation, since it requires two molecules with limited mobility to come in to close proximity of each other. In addition to R.55, Gradwell and McGill ${ }^{102}$ also propose an additional exchange reaction between these pendant groups and MBT to form thiol-pendant groups, which may subsequently result in crosslinks by reacting with benzothiazole-terminated pendant groups. Specifically,

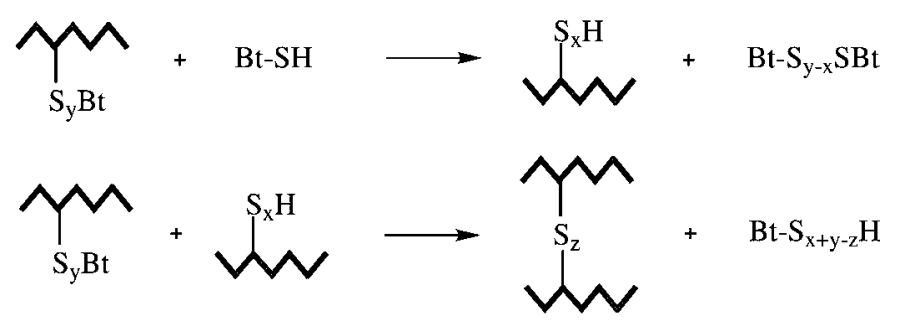

These reactions may be mechanistically reasonable; however, no direct experimental evidence is presently available. In our opinion, it is again difficult to believe that R.56 is the dominant crosslinking reaction, since it requires the two reactive groups that are both attached to polymer chains to diffuse into sufficient proximity for reaction.

In summary, scorch delay is an important characteristic of the vulcanization process and an understanding of the underlying mechanism has significant technological implications. Two mechanisms for scorch delay have been proposed - the thermal stability of the accelerator and alternatively exchange reactions between accelerator polysulfides and other reaction intermediates. We believe that the latter is the dominant mechanism for scorch delay. It is difficult to explain via the thermal stability argument how the observed increase in scorch delay for sulfenamide accelerators increases with the accelerator concentration. ${ }^{22,23,77}$ Specifically, the thermal stability of the accelerator does not depend on the concentration of the accelerator; consequently, if this were the controlling mechanism, the observed delay would be independent of the accelerator concentration, which does not agree with the experimental data. This does not mean that the thermal stability of the accelerator does not provide some measure of scorch delay; rather, that exchange reactions involving the accelerator polysulfides are most probably the controlling mechanism.

\section{POST-CROSSLINKING CHEMISTRY}

The crosslinks that are formed initially are usually polysulfides with high sulfur rank, which subsequently can undergo two competing reactions: (i) Crosslink Desulfuration, which involves rearrangement of the polysulfidic crosslinks to the more stable mono- and disulfidic crosslinks and (ii) Crosslink Decomposition/Degradation where polysulfidic crosslinks degrade to elastically ineffective cyclic sulfides, inactive pendant groups or other main-chain modifications. The reaction rate of these post-crosslinking reactions is higher for the longer length polysulfidic crosslinks, since the S-S bonds are weaker when the crosslinks are longer and therefore it is easier to both desulfurate or degrade the polysulfidic crosslink. The sulfur removed via these two reactions can be used in the formation of new crosslinks or the result in the formation of elastically ineffective cyclic sulfides, pendant groups and main-chain modifications or the formation of $\mathrm{ZnS}^{2}$. Desulfuration and decomposition reactions reflect the two extremes of post-curing reactions and most curing systems will have a combination of both reactions.

1. Crosslink Desulfuration. - Desulfuration involves the removal of sulfur from the longer polysulfidic crosslinks and, eventually leads to the formation of more stable di- and monosulfidic 
crosslinks, ${ }^{97,103-106}$ where the sulfur that is removed from the crosslinks is reused in the vulcanization process to produce additional crosslinks. The shortening of crosslink is often accompanied by changes in the positions of sulfur attachment to the olefin moieties. The polysulfide crosslinks are initially mostly allylic attachments, but these structures are gradually transformed to isoallylic structures as the reaction progresses, where $\mathrm{Zn}(\mathrm{mbt})_{2}$ catalyzes the crosslink shortening and isoallylic transformation reactions. ${ }^{107}$ In order to understand the mechanism of desulfuration in polysulfidic crosslinks, it is instructive to consider the chemistry of model alkenyl sulfides since the polysulfidic crosslinks produced in accelerated sulfur vulcanization are bisalkenyl sulfides. It is well established that triphenylphosphane brings about the desulfuration of organic polysulfides; ${ }^{108,109}$ specifically,

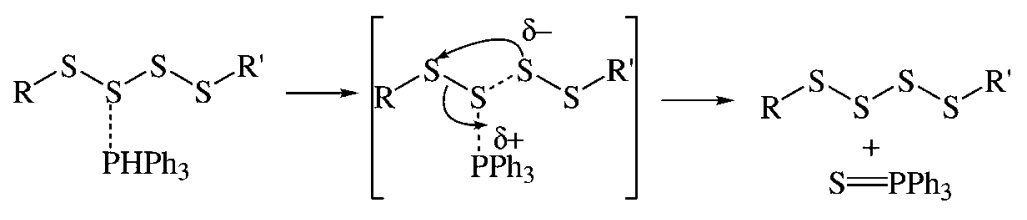

The desulfuration involves nucleophilic attack at the S-S bond by triphenylphosphane followed by the formation of an organothiolate. If $\mathrm{R}$ and $\mathrm{R}^{\prime}$ in $\mathrm{R} .57$ are either alkyl or benzyl, $\mathrm{Ph}_{3} \mathrm{P}$ reduces the polysulfidic chain to a minimum of two sulfur atoms. However when $R$ and $\mathrm{R}^{\prime}$ are alkenyl, the sulfur atoms are reduced to a single sulfur atom via an $\mathrm{S}_{\mathrm{N}}{ }^{\mathrm{i}}$ mechanism involving the rearrangement of the double bond, resulting in a rearranged monosulfide as shown below:

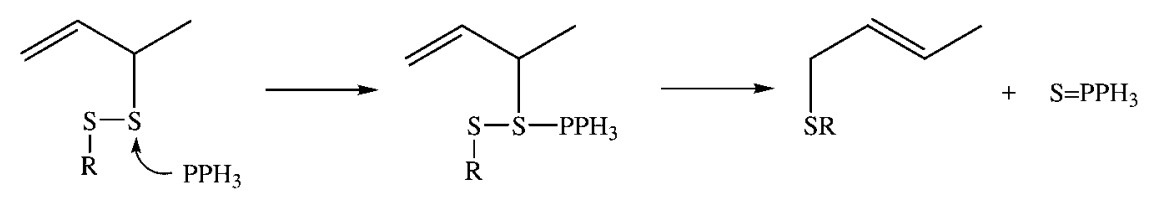

The action of triphenylphosphane on bis-alkenyl sulfides is very similar to the action of ZMBT on the crosslinks, leading Bateman et al. ${ }^{2}$ to conclude that reduction of polysulfidic crosslinks in vulcanized networks also proceeds via an $\mathrm{S}_{\mathrm{N}}{ }^{\mathrm{i}}$ or $\mathrm{S}_{\mathrm{N}}{ }^{{ }^{\prime}}$ mechanism. Specifically, shortening of the polysulfidic chains occurs by the formation of a complex between bis-alkenyl di- or polysulfide and ZMBT, followed by $\mathrm{S}_{\mathrm{N}} \mathrm{i}$ or $\mathrm{S}_{\mathrm{N}} \mathrm{i}^{\prime}$ attack of one of the mercaptide groups on carbon as shown below:

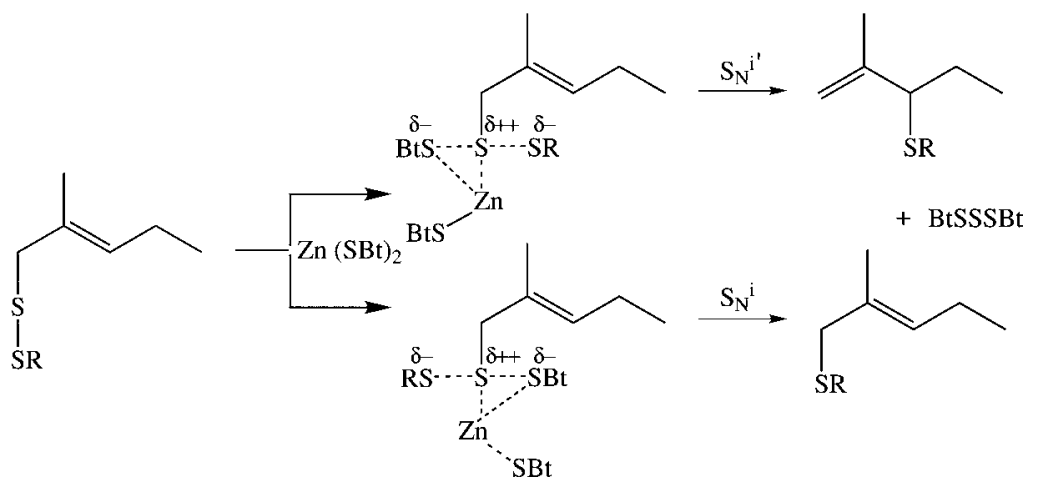

The reaction mechanism assumes that trithio-zinc, and not polythio-zinc, complexes are involved, implying that desulfuration is a stepwise reaction occurring atom by atom (i.e. the 
trisulfide is reduced to disulfide, which in turn is reduced to the monosulfide) and not two sulfur atoms at a time. ${ }^{3}$ With respect to a kinetic analysis, which is the focus of this paper, the two alternate mechanisms in R.59 result in equivalent rate expressions and thus may be generically represented as:

$$
\begin{aligned}
& \mathrm{R}-\mathrm{S}_{\mathrm{x}}-\mathrm{R}+\mathrm{BtS}-\mathrm{Zn}-\mathrm{SBt} \longrightarrow \mathrm{R}-\mathrm{S}_{\mathrm{x}-1}-\mathrm{R}+\mathrm{BtS}-\mathrm{SZn}-\mathrm{SBt} \\
& \mathrm{R}_{-\mathrm{x}-1}-\mathrm{R}+\mathrm{BtS}-\mathrm{Zn}-\mathrm{SBt} \longrightarrow \mathrm{R}-\mathrm{S}_{\mathrm{x}-2}-\mathrm{R}+\mathrm{BtS}-\mathrm{SZn}-\mathrm{SBt}
\end{aligned}
$$

This stepwise reaction scheme will continue until there are only monosulfidic crosslinks left. The regenerated zinc-accelerator complex BS-SZn-SBt will follow the usual route to crosslinks forming crosslink precursors, activated polysulfidic radicals and eventually crosslinks as discussed previously.

2. Crosslink Degradation and Main-Chain-Modifications. - Polysulfidic crosslinks can degrade to dead-ends and main-chain modifications, leading to a net loss of crosslinks, i.e. reversion. By main-chain modifications, we mean the formation of inactive crosslink precursors, cyclic sulfides, conjugated dienes and trienes, as well as the cis-to-trans isomerization of double bonds. Crosslink decomposition is thermally induced, usually at elevated temperatures, ${ }^{97}$ and the mechanism of decomposition may be either radical, polar or a combination of the two. Decomposition of the polysulfidic crosslinks is governed by bond dissociation ${ }^{110}$ and thus the rate of the reaction depends upon the strength of the bond that is broken. ${ }^{111}$ Polysulfides of lengths greater than 4 are most easily broken owing to their lower bond dissociation energies of approximately $150 \mathrm{~kJ} / \mathrm{mol} .{ }^{12}$ The formation of radicals from di- and tri-sulfides is more difficult due to higher dissociation energies involved, which are 289 and $193 \mathrm{~kJ} / \mathrm{mol}$ respectively. This difference in bond dissociation energies is related to the formation of monosulfidic RS ${ }^{\bullet}$ and disulfidic $\mathrm{RS}_{2}{ }^{\bullet}$ radicals, which are not stabilized by neighboring sulfur atoms ${ }^{113}, 114$ like that shown in Structure V. The different products that can be formed from a crosslink are demonstrated for the particular case of a monosulfidic crosslink in R.62 and for the general case of a polysulfidic crosslink in R.63:

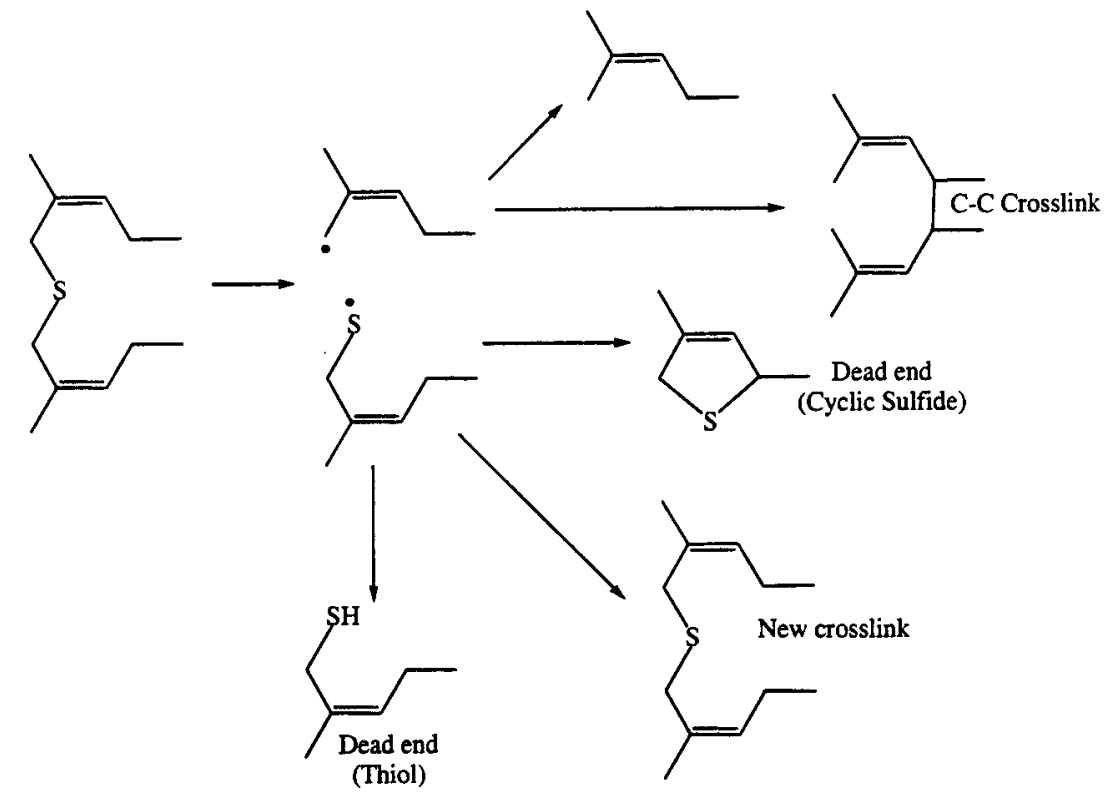


And the potential degradation products that can be formed from a polysulfidic crosslink are:

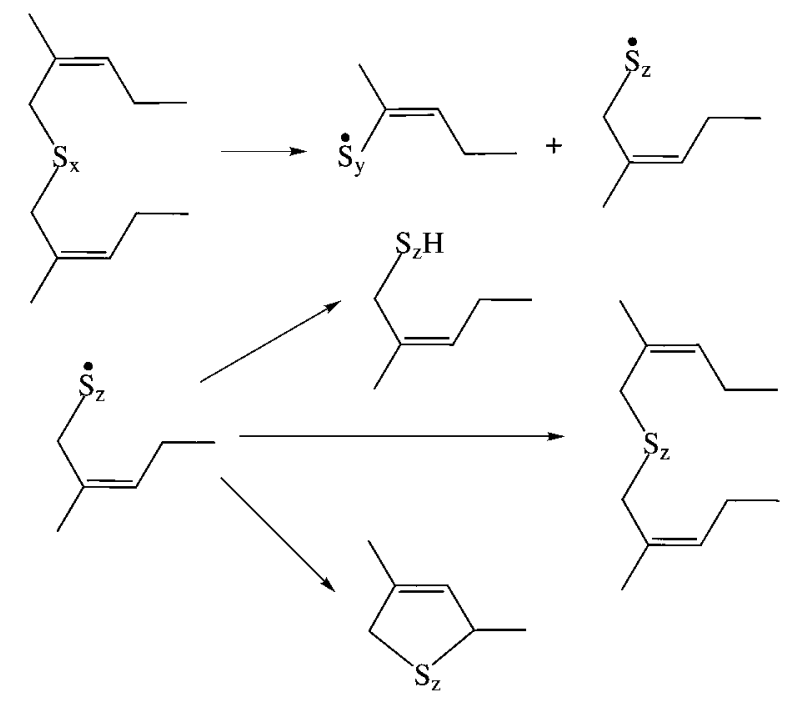

While C-C crosslinks can form in monosulfidic crosslink degradation, such a possibility is rare in a polysulfidic crosslink. Specifically, the degradation of the polysulfidic crosslink $\operatorname{RS}_{\mathrm{y}} \mathrm{R}$ is more likely to proceed via the radicals $\mathrm{RS}_{\mathrm{x}}{ }^{\bullet}$ and $\mathrm{RS}_{\mathrm{z}}{ }^{\bullet}$ than $\mathrm{RS}_{\mathrm{y}}{ }^{\bullet}$ and $\mathrm{R}^{\bullet}$ because the $\mathrm{S}-\mathrm{S}$ bond is more easily broken than the C-S bond. Furthermore, in accordance with the strength of the bond being broken, the overall rate of R.62 should be much slower than the rate of R.63, because of the relative strengths of the monosulfidic S-S bond compared to a polysulfidic S-S bond.

In contrast, it has been proposed that alkyl-alkenyl monosulfides can undergo a polar decomposition mechanism via a cyclic transition state to thioaldehyde and isoprene as shown below via a proton shift in the transition state; $;^{3,115}$ specifically,

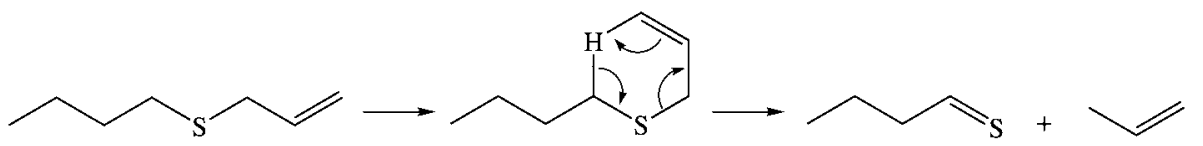

This mode of decomposition might also be present in vulcanized rubber, even at low-to-moderate temperatures. In addition, the decomposition reactions may also involve conjugatively stabilized diene and triene formation and cis-trans isomerization as shown below:
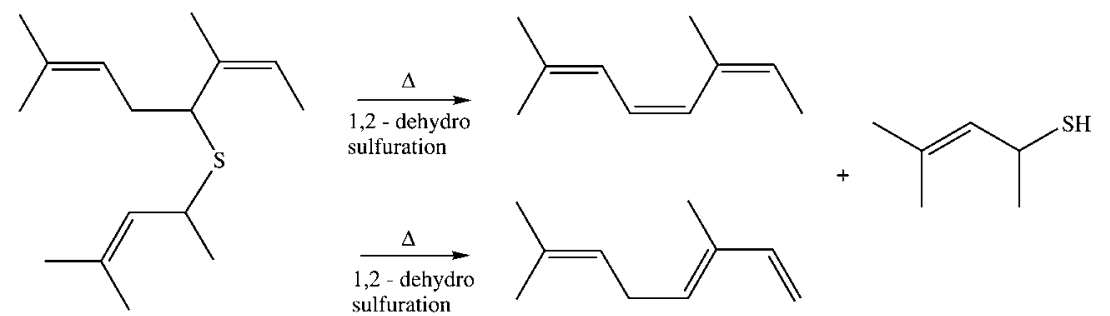

McSweeney and Morrison ${ }^{110}$ found that zinc-complexes promote this type of reactions. The mechanism proposed in R.65 is the 1,2-dehydrosulfuration of monosulfides, but similar reactions should also be considered for polysulfides. Alternatively, one might also consider 1,4-dehydro- 
sulfuration, which leads to the formation of dienes; however, 1,2-dehydrosulfuration will be the preferred reaction due to the formation of stable conjugate triene structures. Chen et al. ${ }^{116}$ found that the reversion process is also associated with cis-to-trans isomerization of the double bonds. Using IR spectroscopy to study the reversion of natural rubber both with and without carbon black, they concluded that the reversion process is related to the formation of trans-methine structures. Zaper and Koenig ${ }^{80,82,117}$ also detected similar structures in their NMR studies of NRvulcanization accelerated with TMTD, MBS and CBS.

The post-crosslinking reactions are important reactions in rubber vulcanization from the perspective of designing engineering parts, since it is the rate and extent of these reactions that ultimately control the life-performance of the vulcanized parts. Although the rate of the postcrosslinking reactions may be slow at use conditions, the time scales for the life of the part may be many years. Because of the considerable cost of directly measuring the response of the vulcanizates during their long service lifetimes, it is critical to have mechanistically viable models of the post-crosslinking reactions. The key reaction mechanisms have been briefly summarized here; however, a more detailed knowledge of the various processes of crosslink shortening and degradation and how they depend on the non-degraded structure of the crosslink is needed in order to more accurately model the life-performance of the engineering vulcanizate.

\section{RETARDER CHEMISTRY}

Until this point, we have discussed the chemistry of accelerated sulfur vulcanized systems without retarders. As discussed in Section III.B.3, scorch delay is an important property of benzothiazole sulfenamide accelerators; however, the delay period provided by these accelerators is typically not sufficient for the necessary pre-cure processing of the rubber in many manufacturing applications. Thus, there is a need for additives that increase the scorch delay time with minimal effects on other aspects of the cure like the cure-rate, time to maximum cure, maximum crosslink density, etc. This increase in scorch delay can be achieved by adding small amounts (i.e. from 0.1 to $0.4 \mathrm{phr}$ ) of a retarder, which is also called a pre-vulcanization inhibitor, PVI. A typical PVI has an R-S-N group, where R does not contain the proton acceptor site common to the accelerator species. A common industrial PVI is N-(cyclohexylthio)pthalimide, commercially known as CTP and its chemical structure is shown below:

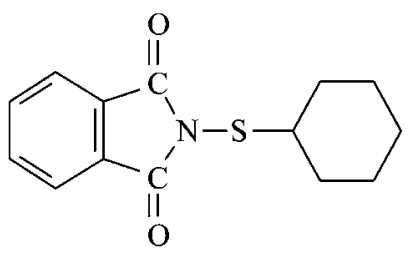

The importance of the S-N bond in the inhibitor molecule has been described in detail by Sullivan et al. ${ }^{118}$ They found that the net reactivity of the PVI would increase by having more S$\mathrm{N}$ leaving groups in the molecule; thus, hexa (cyclohexylthio) melanine with $6 \mathrm{~S}-\mathrm{N}$ groups would have a higher net reactivity than an equal concentration of CTP with 1 S-N group. Nevertheless, CTP remains one of the most industrially important PVIs, since in addition to the net reactivity, the thermal stability of the retarder is an important consideration, and CTP is thermally stable at vulcanization temperatures. Excellent discussions on the reaction mechanisms of retarders are available in the reviews by Trivette et $a l .{ }^{8}$ and Morita. ${ }^{9}$ We will now summarize the salient features in regard to the chemical action of a PVI.

Trivette and Coran ${ }^{119}$ observed that at vulcanization temperatures, organic polysulfides undergo exchange reactions with persulfenyl radicals as shown below, thereby delaying the con- 
version of these radicals into crosslinks. Specifically,

$$
\mathrm{R}_{1}-\mathrm{S}_{\mathrm{x}} \bullet+\mathrm{R}_{2}-\mathrm{S}_{\mathrm{x}+\mathrm{y}}-\mathrm{R}_{2} \longrightarrow \mathrm{R}_{1}-\mathrm{S}_{\mathrm{x}+\mathrm{y}}-\mathrm{R}_{2}+\mathrm{R}_{2}-\mathrm{S}_{\mathrm{x}} \bullet
$$

Consequently, it was proposed that molecules that are capable of quenching the persulfenyl radicals exhibit retarder-like characteristics. As discussed previously with respect to R. 53, accelerator polysulfides are capable of radical quenching; therefore, it is reasonable to expect that retarders would have chemical structures similar to the accelerator and its associated polysulfides. However, unlike the accelerator polysulfides, the retarder and its polysulfides should not readily sulfurate the rubber to yield crosslinks for the retarding action to be operative. This can be achieved by structures that do not contain the critical proton-accepting sites responsible for precursor formation as shown in R.34. The molecule CTP and its polysulfides exhibit both these characteristics and thus it may be concluded that the retarding action of CTP and its polysulfides is due to quenching of the $\mathrm{R}_{1} \mathrm{~S}_{\mathrm{x}}{ }^{\cdot}$ radical. Since the CTP-polysulfides play a critical role in the retarding action, one may ask what reactions lead to their formation. Like their accelerator counterparts, the polysulfides of CTP may be thought to be formed via sulfur activation as shown below in a reaction scheme that is similar to R.21:<smiles>Cc1cccc2c1C(=O)N(SSSSC1CCCCC1)C2=O</smiles>

However, the data of Gradwell and Stephenson ${ }^{120}$ seems to dispute the conclusion that CTPpolysulfides are formed via R.67, since they did not detect any CTP-polysulfides when CTP and sulfur were heated up to $275^{\circ} \mathrm{C}$. If this is the case, it is not clear how CTP-polysulfides would form so that they would subsequently quench the thiyl radicals. Yet, CTP is known to possess excellent retarding action; thus, some other mechanism might be operative, a mechanism which would not require the formation of CTP-polysulfides as intermediates.

The scavenging of MBT by CTP provides such an alternative to R.67, where CTP reacts rapidly with MBT to form CDB and pthalimide as shown below:

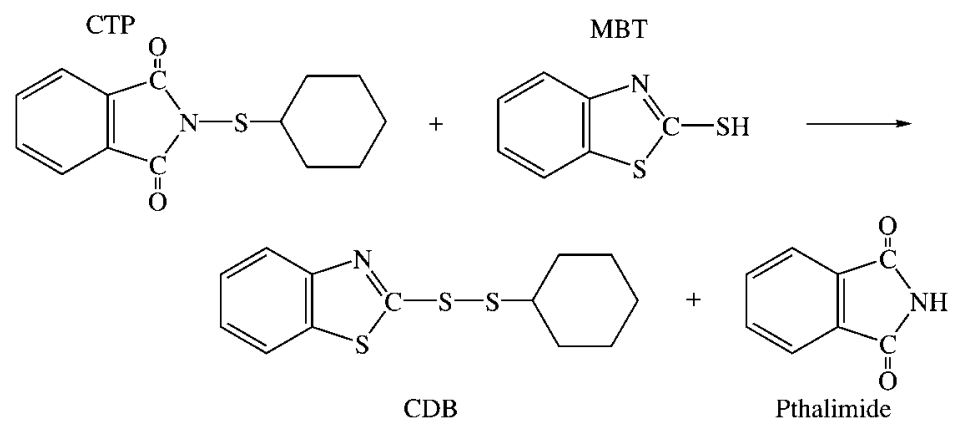

The rate of this reaction is faster than the rate at which MBT reacts with MBS to form MBTS via R.8; thus, MBTS formation is delayed and as a consequence crosslink formation is also delayed. ${ }^{40,121}$ These steps for retarder action are summarized below: 


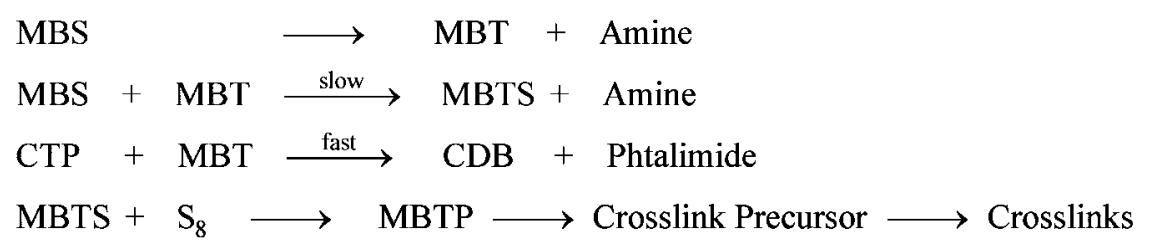

Since the primary function of a retarder is to delay the onset of crosslinking, it should achieve this delay at the minimal cost to other important cure properties. This implies that unless the benzothiazole portion of the accelerator in CDB in R.69 is eventually recycled back in the formation of crosslinks, the cure rate and crosslink density will change with the addition of CTP, since crosslink density is a direct function of the accelerator and sulfur concentrations. CDB is recycled back into the vulcanization system by reacting with MBT to yield MBTS, which can then participate in the formation of crosslinks. Specifically,

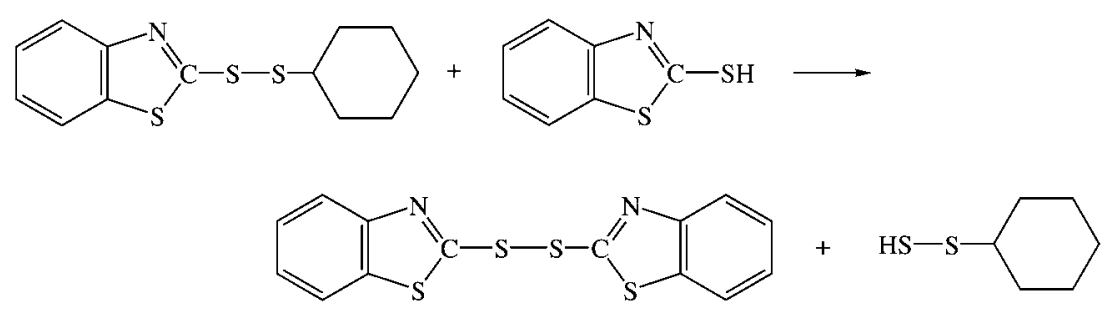

Son et al. ${ }^{40}$ quantitatively studied the reaction of MBT and CTP and reported that the rate of reaction of MBT with CTP was almost five times faster than that of MBT with MBS. The proposed mechanism for the reaction of MBT and CTP is shown below, where the rate-controlling step is the formation of an ion pair shown in resonance structures in R.71a.

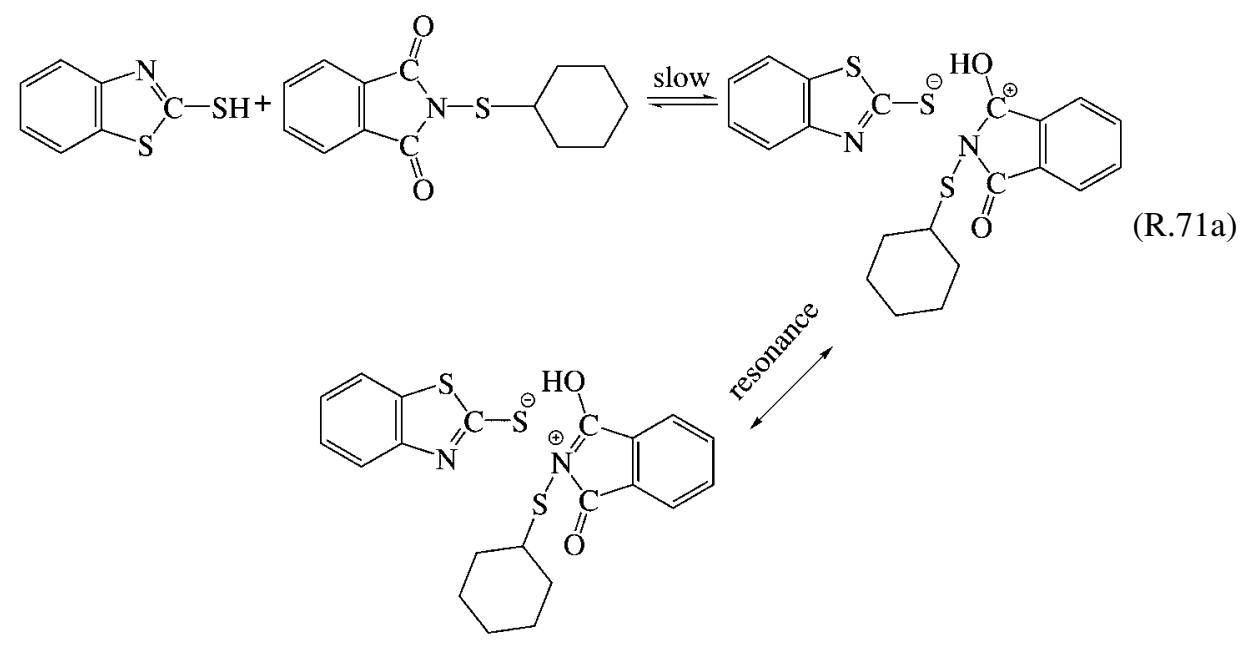




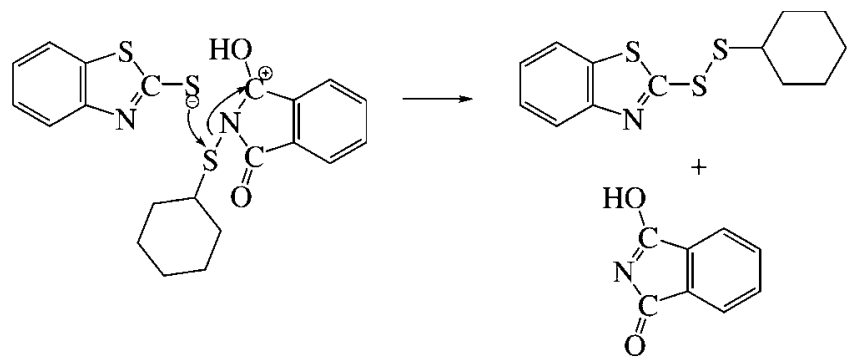<smiles>C[NH+]1C(=O)c2ccccc2C1=O</smiles>

In contrast, Trivette et al. ${ }^{8}$ consider that the formation of the polar complex as proposed by Son et $a l .{ }^{40}$ is unlikely to be the rate-controlling step. They propose that the reaction proceeds via a base catalyzed dissociation of MBT as shown below:

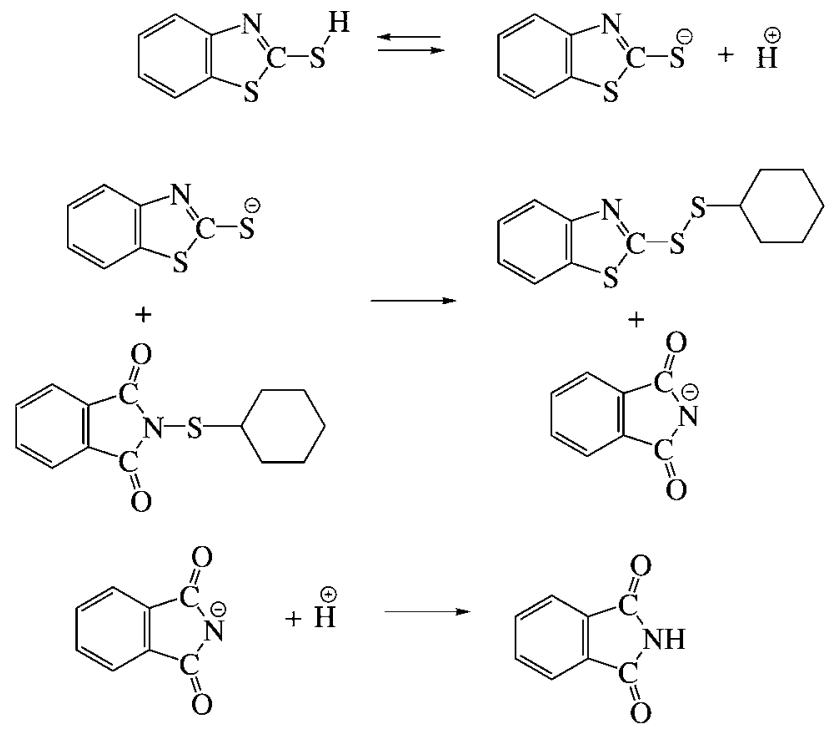

Irrespective of whether the Son et $a l .{ }^{40}$ proposal or Trivette et al. ${ }^{8}$ proposal is the exact mechanism for CTP retardation, it is clear that the CTP-MBT interaction controls the delay action. Thus, MBT must be a key intermediate for retardation by CTP. For MBS accelerated systems, the source of MBT is the initial decomposition of MBS, but for MBTS accelerated systems, there is a disagreement regarding the origin of MBT. Many researchers ${ }^{2,34,81,94,95}$ believe that MBT is created during the formation of crosslink precursors via R.34. However, Gradwell et al. ${ }^{33}$ dispute this conclusion, since they did not observe any MBT during crosslink precursor formation and thus associate the formation of MBT with the crosslinking step. Consequently, they postulate that CTP does not delay the onset of crosslinking, rather it inhibits the crosslinking reactions once 
they have already started. The inhibiting action is due to the rapid reaction of CTP with MBT, making MBT unavailable to catalyze the sulfur activation reaction by MBTS and MBTPs. The scheme makes an implicit assumption that MBT catalyzes the formation of accelerator polysulfides. The essential steps in the proposed scheme of Gradwell and Stephenson ${ }^{120}$ are:

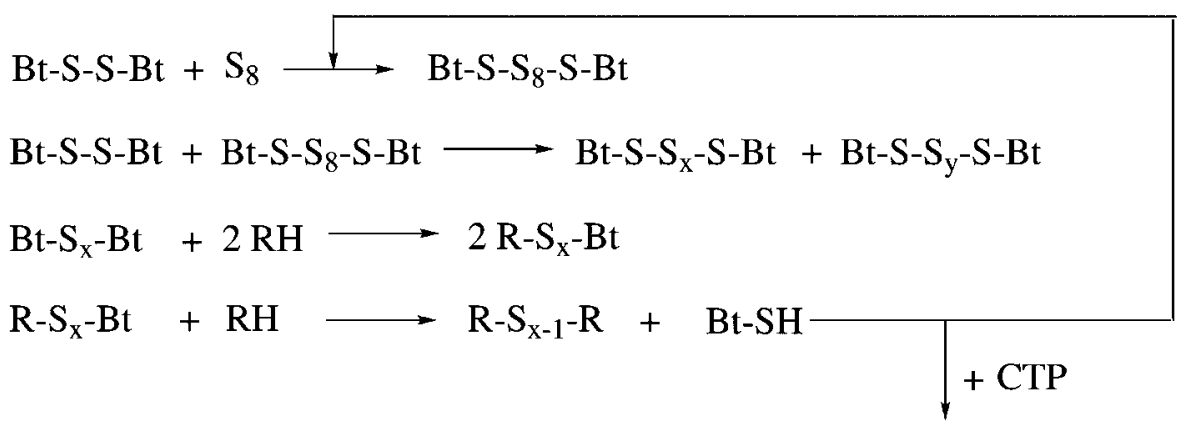

$$
\text { CDB + Pthalimide }
$$

If MBT is removed from the system by CTP, the rate of formation of Bt- $\mathrm{S}_{\mathrm{x}}-\mathrm{Bt}$ species is inhibited. The rate of formation of $\mathrm{BtS}_{\mathrm{x}} \mathrm{Bt}$ will increase again when all the CTP has been used and MBT is again available to catalyze the formation of BtS- $\mathrm{S}_{\mathrm{x}}-\mathrm{SBt}$ species. R.73 is a plausible reaction mechanism that explains the important features of retardation due to CTP and similar species. However, it would be desirable to have direct experimental evidence that CTP retardation only occurs after the initial formation of crosslinks rather than during the formation of crosslink precursors. This type of data would clarify the present disagreement regarding the basic role of a retarder, i.e. does the retarder delay the onset of crosslinking or whether it inhibits the further formation of crosslinks once they have already begun to be formed.

\section{E. CLOSURE}

In this Section we have critically examined the various reaction mechanisms that have been proposed for the chemistry of benzothiazole accelerated sulfur vulcanization. The overall reaction mechanism is summarized in Figure 8. Vulcanization begins with the formation of sulfurating species, which can be zinc-free and/or zinc-complexed accelerators. Although there is agreement on the general molecular structures of these polysulfides, the exact nature of the bonding with zinc is not completely understood; consequently, the relative rate of formation of zinc-free and zinc-complexed polysulfides in zinc activated vulcanization systems is not known. The most important precursor in the formation of these accelerator polysulfides is MBTS, which is formed from the autocatalytic dissociation of MBS (i.e. R.7 and R.8). Accelerator polysulfides are subsequently formed by the direct reaction of lower length accelerator polysulfides with sulfur. It has been proposed that these sulfuration reactions proceed by the insertion of sulfur as $\mathrm{S}_{8}$ molecules (i.e. R.21) or, alternatively, that the sulfuration reactions are sequential, occurring via the insertion of single sulfur atoms (i.e. R.24) which is supported by the data of Morgan and McGill. ${ }^{60}$ A direct consequence of R.24 would be the presence of significant concentration of the species $S_{x}$ for $x$ less than 8 , which have not yet been detected. Also, it is not clear as to what would be the exact chemical structure of the $\mathrm{S}_{\mathrm{x}}$ species, since only $\mathrm{S}_{6}, \mathrm{~S}_{7}$ and $\mathrm{S}_{8}$ can form stable ring structures. In the absence of zinc the sulfuration reactions most probably proceed via a radical mechanism involving the $\mathrm{Bt}-\mathrm{S}_{\mathrm{x}} \cdot$ radical that picks-up sulfur to form longer length radicals; however, in the presence of zinc the sulfuration reactions most probably proceed via a polar 
mechanism (i.e. R.30 and R.31). Since zinc activated systems contain both BtS-S $-\mathrm{SBt}$ and BtS$\mathrm{Zn}_{\mathrm{S}} \mathrm{S}-\mathrm{SBt}$ structures, the sulfuration reactions probably include a combination of radical and polar mechanisms. In addition to the formation of polysulfides by the pick-up of sulfur, the distribution of lengths for accelerator polysulfides can be rearranged via a series of exchange reactions (i.e. R.27 and R.32).

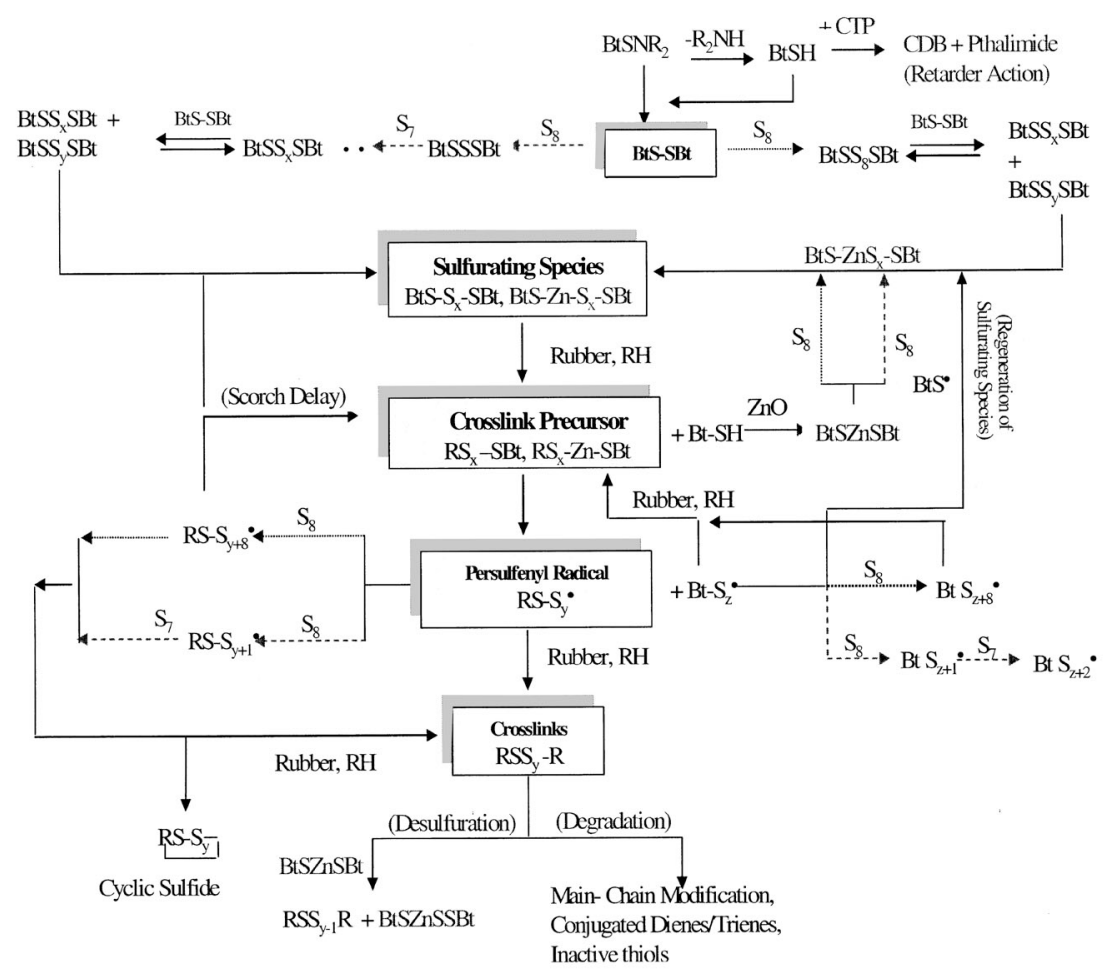

FIG. 8. - Summary of reaction network for benzothiazole sulfenamide accelerated vulcanization of natural rubber. Sulfur pick-up as S8; sequential sulfur pick-up- - - - ; and, either pick-up of sulfur as S8 or sequential sulfur pick-up-

The sulfurating species lead to the formation of crosslink precursors via reaction with the rubber. Like the sulfurating species, the crosslink precursors can include both zinc-free as well as zinc-complexed species. Radical mechanisms have been proposed for the conversion of BtS$\mathrm{S}_{\mathrm{x}}-\mathrm{SBt}$ into crosslink precursors, but in our opinion these mechanisms seem unlikely, since they require the abstraction of hydrogen by the relatively stable $\mathrm{BtS}-\mathrm{S}_{\mathrm{x}}$ - radical to yield the less stable alkenyl radical. A concerted mechanism for the formation of the crosslink precursor (i.e. R.34) seems the more appropriate mechanism for precursor formation. For zinc-complexed sulfurating species, a polar mechanism with the associated liberation of MBT proposed by Bateman et $a .^{2}$ (i.e. R.37) is widely accepted; however, Gradwell et al. ${ }^{33,120}$ question the formation of MBT at this stage of the reaction and argue that the precursor formation in MBS accelerated systems proceeds via the direct reaction of MBS-polysulfides with rubber (i.e. R.36). Although mechanistically plausible, this second scheme assumes that upon heating MBS with sulfur the formation of MBS-polysulfides is dominant rather than the formation of MBTS and its associated polysulfides, which is not observed experimentally.

The crosslink precursors are subsequently converted to crosslinks by (i) the direct reaction of the precursor with another rubber molecule via a persulfenyl intermediate (i.e. R.42 and R.43) 
or (ii) by disproportionation between two crosslink precursors (i.e. R.38 and R.39) or disproportionation between a precursor and the persulfenyl radical (i.e. R.47). Although both the direct and disproportionation reactions are mechanistically plausible, consideration of the diffusional mobility of bulky molecules make the disproportionation reactions for crosslink formation seem unlikely. Once the crosslinks are formed, they can further react by either desulfuration or degradation. The desulfuration reactions are catalyzed by ZMBT and proceed via the removal of one sulfur atom at a time (i.e. R.60 and R.61) and only change the length distribution of crosslinks without changing the total number of crosslinks. In contrast, the degradation reactions lead to loss of crosslinks and results in structures like cyclic sulfides, main-chain modifications and cistrans isomerization, although the details for those reaction mechanisms are not well understood.

Retarders like CTP enhance the scorch delay, because the retarder reacts more rapidly with MBT than the accelerator reacts with MBT (i.e. R.68). This scavenging of MBT by the retarder delays the formation of the critical precursor MBTS from MBS and hence the delay of the vulcanization reaction. Although R.68 is generally accepted as the reaction responsible for the delay, Gradwell et al. ${ }^{33,120}$ argue that MBT is primarily released by the formation of crosslinks (i.e. R.72); thus, the retarder does not delay the initial formation of crosslinks, but only inhibits the rate of crosslink formation.

Although significant questions and some disagreement remain, there is general agreement on most of the key features of the chemistry for benzothiazole accelerated sulfur vulcanization. What is primarily missing is a quantitative analysis that is reasonably faithful to the chemistry. The development of a preliminary set of models for the quantitative description of vulcanization kinetics will be the focus of the remainder of this paper.

\section{POPULATION BALANCE EQUATIONS FOR SULFUR VULCANIZATION}

TABLE IV

Definition of Symbols in the Population Balance Model

\begin{tabular}{lll}
\hline & Name & Chemical Structure \\
\hline $\mathrm{Bt}$ & Benzothiazole group & $\mathrm{C}_{7} \mathrm{H}_{4} \mathrm{NS}$ \\
$\mathrm{A}_{\mathrm{x}}$ & Accelerator polysulfides or & $\mathrm{BtS}-\mathrm{S}_{\mathrm{x}}-\mathrm{SBt}$ or BtS-Zn- $\mathrm{S}_{\mathrm{x}}-\mathrm{SBt}$ \\
& $\quad$ Zn-accelerator complexes & \\
$\mathrm{B}_{0}$ & Inactive crosslink precursor & Rubber-SBt \\
$\mathrm{B}_{\mathrm{x}}$ & Active crosslink precursor & Rubber $-\mathrm{S}_{\mathrm{x}}-\mathrm{SBt}$ \\
$\mathrm{B}^{*}{ }_{\mathrm{x}}$ & Activated form of the crosslink precursor & Rubber $-\mathrm{S}_{\mathrm{x}} \cdot$ \\
$\mathrm{Vu}_{\mathrm{x}}$ & Crosslink & Rubber $-\mathrm{S}_{\mathrm{x}}-$ Rubber \\
$\mathrm{D}_{\mathrm{x}}$ & Dead end (main chain modifications, & \\
& $\quad$ cyclic sulfides of length x, conjugate \\
& $\quad$ diene-triene formation) & \\
$\mathrm{E}_{\mathrm{x}}$ & Accelerated-terminated polysulfidic radical & $\mathrm{BtS}_{\mathrm{x}} \cdot$ \\
$\mathrm{S}_{8}$ & Elemental sulfur & \\
\hline
\end{tabular}

The complex set of reactions that characterize vulcanization chemistry, reactions that involve the formation of sulfurating species, sequential and lumped modes of sulfur activation, formation of crosslink precursors, radical reactions of persulfenyl radicals to form crosslinks and the eventual desulfuration and degradation reactions of the crosslinks were reviewed in Section III. A fundamental kinetic model for vulcanization should explicitly acknowledge these different reactions. This is a substantial task; moreover, additional modeling complexity originates from the polysulfidic nature of the various key intermediates, which must be explicitly incorporated in 
order to completely close the mass balances for each reaction. A lumped modeling approach, like the ones detailed in Section II.C, ignores detailed mass balances and therefore cannot be a fundamental description for describing vulcanization kinetics.

We will develop Population Balance Equations (PBEs) to describe the kinetics of accelerated sulfur vulcanization. As the name population balance suggests, explicit kinetic balances will be written for every member of the population. The population is defined by the collection of sulfurating species of different lengths, crosslink precursors of different lengths, persulfenyl radicals of different types and lengths, crosslinks of different lengths and other participating intermediates. The individual balances for each species will be derived to include all the combinatorial possibilities for the formation and breakage of the different lengths of the polysulfidic bonds. The following assumptions will be made to simplify the analysis; however, all of these assumptions can be relaxed if needed.

1. The concentration of unsaturation sites in natural rubber is assumed to be in considerable excess as compared with the other reactive species. Thus, even though the reac tions with the rubber are inherently second order, they will be modeled as first order reactions where the concentration of rubber will be absorbed into the rate constant. This assumption is valid for elastomers with high degree of unsaturation like natural rubber and SBR but not true for low unsaturation rubbers like butyl rubber. For rubber with low degree of unsaturation, an explicit accounting of the concentration of the unsaturation sites will be required.

2. The rate of formation or breakage of all the S-S bonds in a particular polysulfidic molecule will be assumed to be independent of the position of the bond from the ends of the molecule. For example, the different S-S bonds in BtS-S-S-S-SBt will form or break at the same rate. The assumption of equal reactivity is an obvious first step that can be easily relaxed in the future to acknowledge different reactivities.

3. The reactivity of all the polysulfidic molecules of a particular type will be assumed to be similar for a particular reaction. For example, the reactivity of $\mathrm{E}_{\mathrm{x}}\left(\right.$ i.e. $\left.\mathrm{BtS}-\mathrm{S}_{\mathrm{x}}{ }^{\circ}\right)$ and $\mathrm{B}_{\mathrm{x}}\left(\right.$ i.e. $\mathrm{R}-\mathrm{S}_{\mathrm{x}}{ }^{*}$ ) with an allylic hydrogen on the rubber will be similar. This assumption is consistent with the observation that the exact nature of the organic end groups has minimal effect on the reactivity of species that are well removed from the organic end groups. Here again the PBEs can easily be generalized to acknowledge different reac tivities for different species; however, the current assumption of equal reactivities is a natural starting point.

4. Zinc and zinc-free structures (e.g. BtS-S - -SBt and BtS-Zn-S $-\mathrm{x}_{\mathrm{x}}-\mathrm{SBt}$ or BtS-S $-\mathrm{-}$ and BtS$\mathrm{Zn}-\mathrm{S}_{\mathrm{y}}-\mathrm{Bt}$ ) will not be distinguished explicitly, since both these structures participate in the same reactions. The reaction rates for the zinc and zinc-free structures will be assumed to be the same.

5. The maximum number of sulfur atoms in any polysulfidic species is restricted to $16^{\dagger}$. This includes the active as well as the non-active sulfur atoms. Here, active sulfur atom refers to the sulfur atoms in the structure excluding the BtS- unit. For example BtSSSBt has one active sulfur atom and two inactive sulfur atoms. This limit is set for computational efficiency and can be readily increased; however, for the systems studied in this paper, there was no need to consider polysulfides species longer than 16.

The nomenclature for various species considered in the PBEs is given in Table IV, except for obvious species like MBS, CTP, $\mathrm{S}_{8}$, etc. which are not included. The different polysulfidic species considered are the sulfurating species $A_{x}$, the crosslink precursors $B_{x}$, the persulfenyl radicals $\mathrm{B}_{\mathrm{x}}{ }_{\mathrm{x}}$, the crosslinks $\mathrm{Vu}_{\mathrm{x}}$ and the dead-ends $\mathrm{D}_{\mathrm{x}}$. A basic building block for the different intermediates in the PBEs is BtS- where $\mathrm{Bt}$ is the benzothiazole group and $\mathrm{S}$ is the sulfur atom

$\dagger$ Exceptions are $\mathrm{B}_{16}$ and $\mathrm{E}^{*}{ }_{16}$ which have a total of $17 \mathrm{~S}$-atoms. $\mathrm{B}_{16}$ is required since it fragments to give $\mathrm{B} *{ }_{16}$, which is the only species that will yield $\mathrm{Vu}_{16} . \mathrm{E}^{*}{ }_{16}$ is required because it is the only species that can form $\mathrm{B}_{16}$. 
attached to the benzothiazole group via the C-S bond. Since the C-S bond is a much stronger bond than S-S bond, we will assume that it does not fragment and hence is the lowest level of detail in the model. The subscript $\mathrm{x}$ on these species denotes the number of active sulfur atoms in the structure. For example, MBTS, has no active sulfur atom and hence will be denoted by $\mathrm{A}_{0}$, while the accelerator-polysulfide with structure $\mathrm{BtS}-\mathrm{S}_{\mathrm{x}}-\mathrm{SBt}$ will be denoted by $\mathrm{A}_{\mathrm{x}}$.

We will now show how the PBEs are developed for two specific reactions. First consider the reaction between the BtS- $\mathrm{S}_{2}-\mathrm{SBt}$ accelerator (i.e. $\mathrm{A}_{2}$ ) and rubber to form crosslink precursors along with the release of MBT and BtSSH:

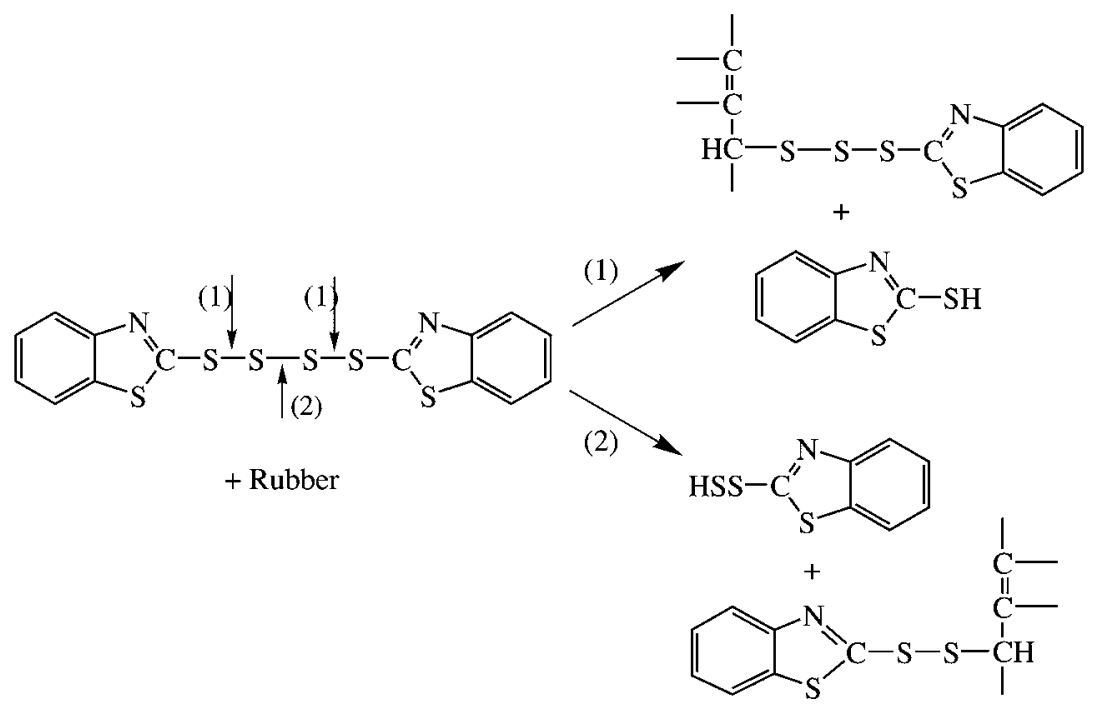

In $\mathrm{A}_{2}$, there are two different types of sulfur-sulfur bonds that can break with associated rate constants $\mathrm{k}_{\mathrm{A}-\mathrm{R}}{ }^{(1)}$ and $\mathrm{k}_{\mathrm{A}-\mathrm{R}}{ }^{(2)}$. The $\mathrm{C}-\mathrm{S}$ bond next to the benzothiazole moiety will not break because of its higher stability as compared to the S-S bond. The crosslink precursor $\mathrm{B}_{2}$ formed from R.74 will subsequently yield persulfenyl radicals (i.e. $\mathrm{B}_{1}^{*}, \mathrm{~B}_{2}{ }_{2}, \mathrm{E}_{0}{ }_{0}$ and $\mathrm{E}_{1}^{*}$ ) as shown below:

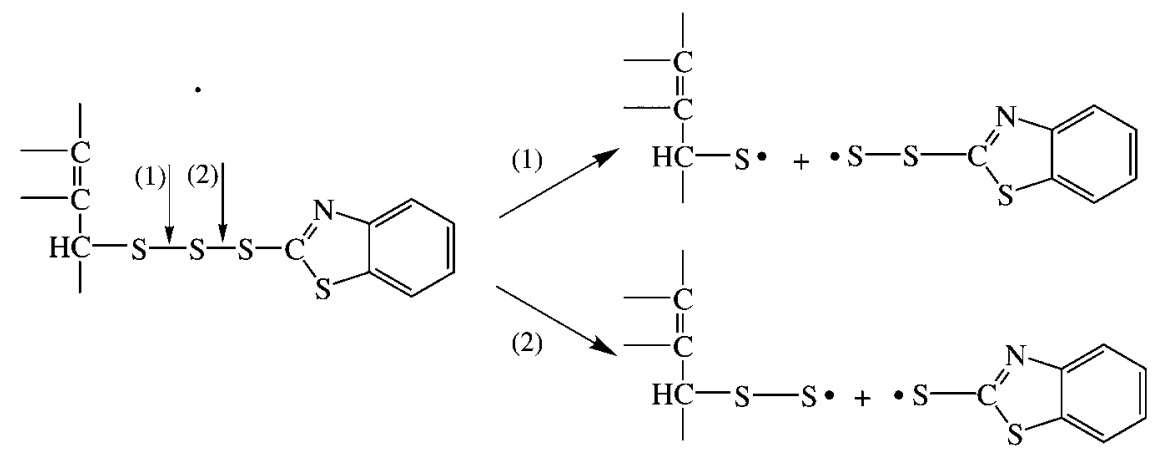

There are two potential bond-breaking sites having the rate constants $\mathrm{k}_{\mathrm{B}-\mathrm{R}}{ }^{(1)}$ and $\mathrm{k}_{\mathrm{B}-\mathrm{R}}{ }^{(2)}$. Depending on the site at which the bond breaks, either $\mathrm{B}_{1}^{*}$ and $\mathrm{E}_{1}^{*}$ or $\mathrm{B}_{2}{ }_{2}$ and $\mathrm{E}_{0}^{*}$ is formed. Thus, the contributions to the population balance equations from just R.74 and R.75 for the $\mathrm{A}_{2}$ and $\mathrm{B}_{2}$ molecules are: 


$$
\begin{gathered}
\frac{\mathrm{d}\left[\mathrm{A}_{2}\right]}{\mathrm{dt}}=-\left(2 \mathrm{k}_{\mathrm{A}-\mathrm{R}}^{(1)}+\mathrm{k}_{\mathrm{A}-\mathrm{R}}^{(2)}\right)\left[\mathrm{A}_{2}\right] \\
\frac{\mathrm{d}\left[\mathrm{B}_{2}\right]}{\mathrm{dt}}=\left(2 \mathrm{k}_{\mathrm{A}-\mathrm{R}}^{(1)}+\mathrm{k}_{\mathrm{A}-\mathrm{R}}^{(2)}\right)\left[\mathrm{A}_{2}\right]-\left(\mathrm{k}_{\mathrm{B}-\mathrm{R}}^{(1)}+\mathrm{k}_{\mathrm{B}-\mathrm{R}}^{(2)}\right)\left[\mathrm{B}_{2}\right]
\end{gathered}
$$

The rate constants may be related to the strength of the bond being broken via the wellknown Polanyi relation; ${ }^{122}$ however, the assumption of a Polanyi relationship is not an essential feature of the development of the PB equations. There are simplifying assumptions that we can apply. Specifically, as per the discussion in Section III.B.1 with respect to R.34, the formation of $\mathrm{BtSH}$ is much more likely then the formation of BtSSH for zinc-free systems. Moreover, the concerted mechanism given in R.34 will be more likely to proceed with small ring transition state structures than for larger ring structures; consequently, we anticipate that $\mathrm{k}_{\mathrm{A}-\mathrm{R}}{ }^{(1)}>>\mathrm{k}_{\mathrm{A}-\mathrm{R}}{ }^{(2)}$. Also, according to Assumption 2, similar bonds within the same molecule, like the two different S-S bonds in the $\mathrm{B}_{2}$ crosslink precursor, are assumed to have equal reactivities. Thus, $\mathrm{k}_{\mathrm{A}-\mathrm{R}}{ }^{(1)}=\mathrm{k}_{\mathrm{A}-\mathrm{R}}$, $\mathrm{k}_{\mathrm{A}-\mathrm{R}}{ }^{(2)}=0$ and $\mathrm{k}_{\mathrm{B}-\mathrm{R}}{ }^{(1)}=\mathrm{k}_{\mathrm{B}-\mathrm{R}}{ }^{(2)}=\mathrm{k}_{\mathrm{B}-\mathrm{R}}$; of course, these restrictions on the rate constants can be relaxed if needed in order to describe the vulcanization data. Similar balances on the species involved in R.74 and R.75 are:

$$
\begin{aligned}
& \frac{\mathrm{d}\left[\mathrm{B} *_{1}\right]}{\mathrm{dt}}=\frac{\mathrm{d}\left[\mathrm{B} *_{2}\right]}{\mathrm{dt}}=\mathrm{k}_{\mathrm{B}-\mathrm{R}}\left[\mathrm{B}_{2}\right] \\
& \frac{\mathrm{d}\left[\mathrm{E}_{0}^{*}{ }_{0}\right]}{\mathrm{dt}}=\frac{\mathrm{d}\left[\mathrm{E}_{1}^{*}{ }_{1}\right]}{\mathrm{dt}}=\mathrm{k}_{\mathrm{B}-\mathrm{R}}\left[\mathrm{B}_{2}\right] \\
& \frac{\mathrm{d}[\mathrm{MBT}]}{\mathrm{dt}}=2 \mathrm{k}_{\mathrm{A}-\mathrm{R}}\left[\mathrm{A}_{2}\right]
\end{aligned}
$$

There will of course be reactions other than R.74 and R.75 that contribute to the generation or depletion of the various species and all these contributions will need to be added to Equations 3 and 4. Using analogous methodology as that outlined in this example, the governing differential balances for all the species involved in the chemistry of sulfur vulcanization will now be developed. To facilitate the ease of development of these balances, the reaction network of Figure 8 is summarized in tabular form in Tables V and VI. The reaction schemes in Tables V and VI are identical, except for the mechanism by which sulfur is inserted in the network. In the reaction scheme of Table V, which we will hereafter refer to as Model-I, it is assumed that different species pick-up sulfur in the form of $\mathrm{S}_{8}$ molecules (see reactions 3,10 and 12 in Table V); in contrast, for the reaction scheme given in Table VI, which will be referred to as Model-II, the insertion of sulfur is assumed to be sequential. The associated rate constants for the different reactions are also shown in these Tables.

We are now ready to develop the PBEs. The remainder of this Section will be organized as follows: In Section IV.A, the PBEs will be developed for sulfur vulcanization accelerated by benzothiazole sulfenamides for the situation when sulfur is added to the accelerator as $S_{8}$, while in Section IV.B, the analogous PBEs will be developed for the situation where sulfur is sequentially added to the accelerator as single $S$ atoms. The difference between the two proposed modes of sulfur pick-up is one of the major areas of disagreement in the reaction mechanisms as discussed in Section III. In Section IV.A and IV.B, the PBEs will be developed for the situation where no retarder is present. When retarder is present, the PBEs are altered slightly as will be presented in Section IV.C. 
TABLE V

Reaction Scheme and the Associated Rate-Constants Considered in Model-I: Sulfur ACtivation is By $\mathrm{S}_{8}$ PickuP

\begin{tabular}{|c|c|c|c|}
\hline $\begin{array}{l}\text { Reaction } \\
\text { Number }\end{array}$ & Reaction Type & $\begin{array}{c}\text { Rate } \\
\text { Constant } \\
\end{array}$ & $\begin{array}{c}\text { Reaction } \\
\text { Mechanism }\end{array}$ \\
\hline & Accelerator Chemistry & & \\
\hline 1 & $\mathrm{MBS} \rightarrow \mathrm{MBT}+$ Amine & $\mathrm{k}_{\text {MBS }}$ & R.7 \\
\hline 2 & $\mathrm{MBS}+\mathrm{MBT} \rightarrow \mathrm{A}_{0}+$ Amine & $\mathrm{k}_{\mathrm{MBS}^{-}{ }^{-\mathrm{MBT}}}$ & R. 8 \\
\hline 3 & $A_{x}+S_{8} \rightarrow A_{x+8}: 0 \leq x \leq 6$ & $\mathrm{k}_{\mathrm{A}-\mathrm{S}}$ & R.21 \\
\hline \multirow[t]{2}{*}{4} & $A_{0}+A_{x} \leftrightarrow \quad A_{y}+A_{z}: x=y+z$ and $1 \leq x, y, z \leq 14$ & $\mathrm{k}_{\mathrm{A}-\mathrm{A}}$ & R.27 \\
\hline & Crosslinking Chemistry & & \\
\hline 5 & $\mathrm{~A}_{\mathrm{x}}+$ Rubber $\rightarrow \mathrm{B}_{\mathrm{x}}+\mathrm{MBT}: 0 \leq \mathrm{x} \leq 14$ & $\mathrm{k}_{\mathrm{A} \cdot \mathrm{R}}$ & R.34 \\
\hline 6 & $B_{x} \rightarrow B_{y}^{*}+E_{z}^{*}: 1 \leq x, y, z \leq 16$ & $\mathrm{k}_{\mathrm{B}-\mathrm{R}}$ & R. 42 \\
\hline \multirow[t]{2}{*}{7} & $\mathrm{~B}^{*}{ }_{\mathrm{x}}+$ Rubber $\rightarrow \mathrm{Vu}_{\mathrm{x}}: 1 \leq \mathrm{x} \leq 16$ & $\mathrm{kvu}_{\mathrm{vu}}$ & R.43 \\
\hline & Post-Crosslinking Chemistry & & \\
\hline 8 & $\mathrm{Vu}_{\mathrm{x}} \rightarrow$ Dead Ends $: 1 \leq \mathrm{x} \leq 16$ & $k_{\mathrm{DEG}}$ & R.62, R.63 \\
\hline \multirow[t]{2}{*}{9} & $V u_{x}+A_{0} \rightarrow V u_{x-1}+A_{1}: 1 \leq x \leq 16$ & $k_{\text {DESULF }}$ & R.60, R.61 \\
\hline & Other Reactions & & \\
\hline 10 & $\mathrm{E}^{*}{ }_{\mathrm{x}}+\mathrm{S}_{8} \rightarrow \mathrm{E}_{\mathrm{x}+8}: 0 \leq \mathrm{x} \leq 8$ & $k_{E-S}$ & R. 19 \\
\hline 11 & $E^{*}{ }_{x}+$ Rubber $\rightarrow \quad B_{x}: 0 \leq x \leq 16$ & $\mathrm{k}_{\mathrm{E}-\mathrm{R}}$ & R.49 \\
\hline 12 & $\mathrm{~B}^{*}{ }_{\mathrm{x}}+\mathrm{S}_{8} \rightarrow \mathrm{B}^{*}{ }_{\mathrm{x}+8}: 1 \leq \mathrm{x} \leq 8$ & $k_{\text {BST }-\mathrm{S}}$ & R.50 \\
\hline 13 & $\mathrm{~B}^{*}{ }_{\mathrm{x}}+$ Rubber $\rightarrow \quad \mathrm{L}_{\mathrm{x}}: 1 \leq \mathrm{x} \leq 16$ & $\mathrm{k}_{\mathrm{LOOP}}$ & R.52 \\
\hline 14 & $\mathrm{~B}_{\mathrm{x}}^{*}+\mathrm{A}_{0} \rightarrow \mathrm{B}_{\mathrm{x}}+\mathrm{E}_{0}: 1 \leq \mathrm{x} \leq 16$ & $\mathrm{k}_{\mathrm{A}-\mathrm{BST}}$ & R.53 \\
\hline 15 & $\mathrm{E}^{*}{ }_{\mathrm{x}}+\mathrm{E}^{*_{0}} \rightarrow \mathrm{A}_{\mathrm{x}} \quad: 1 \leq \mathrm{x} \leq 14$ & $k_{\mathrm{E}-\mathrm{E}}$ & R.16 \\
\hline
\end{tabular}


TABLE VI

Reaction Scheme and the Associated Rate-Constants Considered in Model-II: Sulfur Activation is Sequential

\begin{tabular}{|c|c|c|c|}
\hline $\begin{array}{l}\text { Reaction } \\
\text { Number }\end{array}$ & Reaction Type & $\begin{array}{c}\text { Rate } \\
\text { Constant }\end{array}$ & $\begin{array}{c}\text { Reaction } \\
\text { Mechanism }\end{array}$ \\
\hline & Accelerator Chemistry & & \\
\hline 1 & $\mathrm{MBS} \rightarrow \mathrm{MBT}+$ Amine & $k_{\text {MBS }}$ & R.7 \\
\hline 2 & $\mathrm{MBS}+\mathrm{MBT} \rightarrow \mathrm{A}_{0}+$ Amine & $\mathrm{k}_{\mathrm{MBS}-\mathrm{MBT}}$ & R. 8 \\
\hline 3 & $A_{x}+S_{y} \rightarrow A_{x+1}+S_{y-1}: 0 \leq x \leq 14,1 \leq y \leq 8$ & $\mathrm{k}_{\mathrm{A}-\mathrm{S}}$ & R.24 \\
\hline \multirow[t]{2}{*}{4} & $A_{0}+A_{x} \longleftrightarrow A_{y}+A_{z}: x=y+z$ and $1 \leq x, y, z \leq 14$ & $\mathrm{k}_{\mathrm{A} \cdot \mathrm{A}}$ & R. 27 \\
\hline & Crosslinking Chemistry & & \\
\hline 5 & $\mathrm{~A}_{\mathrm{x}}+$ Rubber $\rightarrow \mathrm{B}_{\mathrm{x}}+\mathrm{MBT}: 0 \leq \mathrm{x} \leq 14$ & $\mathrm{k}_{\mathrm{A}-\mathrm{R}}$ & R. 34 \\
\hline 6 & $\mathrm{~B}_{\mathrm{x}} \quad \rightarrow \quad \mathrm{B}_{\mathrm{y}}{ }+\mathrm{E}_{\mathrm{z}}^{*}: 1 \leq \mathrm{x}, \mathrm{y}, \mathrm{z} \leq 16$ & $\mathrm{k}_{\mathrm{B}-\mathrm{R}}$ & R.42 \\
\hline \multirow[t]{2}{*}{7} & $\mathrm{~B}^{*}{ }_{\mathrm{x}}+$ Rubber $\rightarrow \mathrm{Vu}_{\mathrm{x}}: 1 \leq \mathrm{x} \leq 16$ & $\mathbf{k}_{\mathrm{vu}}$ & R.43 \\
\hline & Post-Crosslinking Chemistry & & \\
\hline 8 & $\mathrm{Vu}_{\mathrm{x}} \rightarrow$ Dead Ends $: 1 \leq \mathrm{x} \leq 16$ & $\mathrm{k}_{\mathrm{DEG}}$ & R.62, R.63 \\
\hline \multirow[t]{2}{*}{9} & $\mathrm{Vu}_{\mathrm{x}}+\mathrm{A}_{0} \rightarrow \mathrm{Vu}_{\mathrm{x}-1}+\mathrm{A}_{1}: 1 \leq \mathrm{x} \leq 16$ & $k_{\text {DESULF }}$ & R.60, R.61 \\
\hline & Other Reactions & & \\
\hline 10 & $E^{*}{ }_{x}+S_{y} \rightarrow E_{x+1}^{*}+S_{y-1}: 1 \leq x \leq 16,1 \leq y \leq 8$ & $\mathrm{k}_{\mathrm{E}-\mathrm{S}}$ & R.23 \\
\hline 11 & $\mathrm{E}_{\mathrm{x}}^{*}+$ Rubber $\rightarrow \quad \mathrm{B}_{\mathrm{x}}: 0 \leq \mathrm{x} \leq 16$ & $\mathrm{k}_{\mathrm{E}-\mathrm{R}}$ & R.49 \\
\hline 12 & $\mathrm{~B}^{*}{ }_{\mathrm{x}}+\mathrm{S}_{8} \rightarrow \mathrm{B}^{*}{ }_{\mathrm{x}+1}+\mathrm{S}_{\mathrm{y}-1}: 1 \leq \mathrm{x} \leq 16,1 \leq \mathrm{y} \leq 8$ & $\mathbf{k}_{\text {BST-S }}$ & R.51 \\
\hline 13 & $\mathrm{~B}^{*}{ }_{x}+$ Rubber $\rightarrow L_{x}: 1 \leq x \leq 16$ & $\mathrm{k}_{\mathrm{LOOP}}$ & R.52 \\
\hline 14 & $\mathrm{~B}^{*}{ }_{\mathrm{x}}+\mathrm{A}_{0} \rightarrow \mathrm{B}_{\mathrm{x}}+\mathrm{E}_{0}: 1 \leq \mathrm{x} \leq 16$ & $\mathrm{k}_{\mathrm{A} \cdot \mathrm{BST}}$ & R. 53 \\
\hline 15 & $E^{*}{ }_{x}+E^{*}{ }_{0} \rightarrow A_{x} \quad: 1 \leq x \leq 14$ & $\mathrm{k}_{\mathrm{E}-\mathrm{E}}$ & R.16 \\
\hline
\end{tabular}

\section{A. POPULATION BALANCE EQUATIONS FOR MODEL-I: SULFUR PICK-UP AS $\mathrm{S}_{8}$}

We will now develop the full set of PBEs for the situation where sulfur is picked-up by the accelerator as an $\mathrm{S}_{8}$ entity. This is one of the proposed modes of sulfur pick-up and it has significant mechanistic consequences, since it determines the distribution of polysulfidic chain lengths in the activating species. The distribution of chain lengths of all subsequent species in the vulcanization process depends upon the chain length distribution in the activating species. The overall reaction scheme is shown in Figure 8, and a summary of the relevant reaction mechanisms for Model-I are given in Table V. The first species that must be considered in the vulcanization process is the accelerator, MBS. MBS is converted to MBTS via R.7 and R.8, which yield 
the following kinetic equation:

$$
\frac{\mathrm{d}}{\mathrm{dt}}[\mathrm{MBS}]=-\mathrm{k}_{\mathrm{MBS}}[\mathrm{MBS}]-\mathrm{k}_{\mathrm{MBS}-\mathrm{MBT}}[\mathrm{MBT}][\mathrm{MBS}]
$$

Next the balance equations for the sulfurating species will be developed for the chemistry that was detailed in Section III.A. Both BtS-S $-\mathrm{SBt}$ and BtS-Zn- $\mathrm{S}_{\mathrm{x}}-\mathrm{SBt}$ are sulfurating species, however by Assumption 4 the reactivities for both species is assumed to be the same; thus, $A_{x}$ is defined to include both the zinc and zinc-free species. The first species that must be considered in $\mathrm{A}_{\mathrm{x}}$ is MBTS, which is $\mathrm{A}_{0}$ in our notation. $\mathrm{A}_{0}$ is initially formed via $\mathrm{R} .8$ through the autocatalytic action of MBT on MBS and consumed by (i) pick-up of sulfur via R.21, (ii) exchange reactions with other sulfurating species as given in R.27 (iii) formation of an inactive crosslink precursor as specified in R.34, (iv) radical quenching of the persulfenyl radicals via R.53 and finally by the desulfuration reaction in the reversion chemistry shown in R.60 and $61 . \mathrm{A}_{0}$ can also be regenerated by combination of two $\mathrm{E}^{*}{ }_{0}$ radicals as shown in R.16. Thus, the complete balance on $\mathrm{A}_{0}$ yields

$$
\begin{aligned}
\frac{\mathrm{d}}{\mathrm{dt}}\left[\mathrm{A}_{0}\right] & =\mathrm{k}_{\mathrm{MBS}-\mathrm{MBT}}[\mathrm{MBT}][\mathrm{MBS}]-\mathrm{k}_{\mathrm{A}-\mathrm{A}}\left[\mathrm{A}_{0}\right] \sum_{\mathrm{r}=2}^{14}(\mathrm{r}-1) \mathrm{A}_{\mathrm{r}}-\mathrm{k}_{\mathrm{A}-\mathrm{S}}\left[\mathrm{A}_{0}\right]\left[\mathrm{S}_{8}\right] \\
& -\mathrm{k}_{\mathrm{A}-\mathrm{R}}\left[\mathrm{A}_{0}\right]-\mathrm{k}_{\mathrm{A}-\mathrm{BST}}\left[\mathrm{A}_{0}\right] \sum_{\mathrm{r}=1}^{16} \mathrm{~B}_{\mathrm{r}}{ }_{\mathrm{r}}+\frac{1}{2} \mathrm{k}_{\mathrm{E}-\mathrm{E}}\left[\mathrm{E}_{0}{ }_{0}\right]^{2}-\mathrm{k}_{\text {DESULF }}\left[\mathrm{A}_{0}\right] \sum_{\mathrm{r}=2}^{16} \mathrm{Vu}_{\mathrm{r}} \\
& +\mathrm{k}_{\mathrm{A}-\mathrm{A}} \sum_{\mathrm{x}=1}^{13} \sum_{\mathrm{y}=1}^{13-\mathrm{x}} \mathrm{A}_{\mathrm{x}} \mathrm{A}_{\mathrm{y}}
\end{aligned}
$$

The balance equations for the other sulfurating species will now be developed using the following simplifications:

1. Since the longest polysulfidic species is restricted to a length of 16 , it follows that $A_{14}$ is the longest $A_{x}$. Thus, $A_{x}$ can react with $S_{8}$ to give $A_{x+8}$ for $x \leq 6$.

2. Exchange reactions between the different sulfurating species are given by R.27.

However the exchange reactions depend on the relative mobility of the sulfurating species of different lengths and their relative abundance. Since $\mathrm{A}_{0}$ is the most mobile and the most abundant sulfurating species, it will be assumed that the dominant exchange reactions are the ones in which $\mathrm{A}_{0}$ participates. Thus the general scheme given by R.27 will be modeled as shown below:

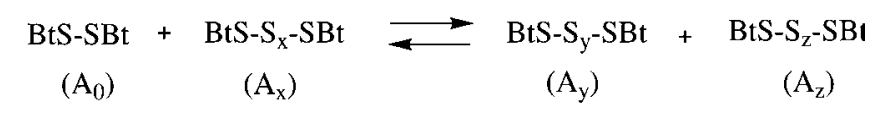

where $\mathrm{x}=\mathrm{y}+\mathrm{z}$ in order to close the sulfur balance. In addition, it will be assumed that the rate of forward and the backward reactions are the same since both reactions are essentially sulfur-exchange reactions.

3. Using similar arguments as those in point 2 above, the recombination of the $\mathrm{E}_{\mathrm{x}}^{*}$ benzothiazole radicals to yield $\mathrm{A}_{\mathrm{x}}$ will be restricted to the reactions of the type: 


$$
\begin{aligned}
& \mathrm{BtS}^{\bullet}+\mathrm{BtS}-\mathrm{S}_{\mathrm{x}}^{\bullet} \longrightarrow \mathrm{BtS}-\mathrm{S}_{\mathrm{x}}-\mathrm{SBt} \\
& \left(\mathrm{E}^{*}{ }_{0}\right) \quad\left(\mathrm{E}^{*}{ }_{\mathrm{x}}\right) \quad\left(\mathrm{A}_{\mathrm{x}}\right)
\end{aligned}
$$

Considering R.16, R.21, R.27, R.34, R.53 and R.60 for the case $x=1$, the PBE for $A_{1}$ is given by:

$$
\begin{aligned}
\frac{\mathrm{d}}{\mathrm{dt}}\left[\mathrm{A}_{1}\right]= & -2 \mathrm{k}_{\mathrm{A}-\mathrm{R}}\left[\mathrm{A}_{1}\right]-\mathrm{k}_{\mathrm{A}-\mathrm{S}}\left[\mathrm{A}_{1}\right]\left[\mathrm{S}_{8}\right]+2 \mathrm{k}_{\mathrm{A}-\mathrm{A}}\left[\mathrm{A}_{0}\right] \sum_{\mathrm{r}=2}^{14} \mathrm{~A}_{\mathrm{r}}+\mathrm{k}_{\text {DESULF }}\left[\mathrm{A}_{0}\right] \sum_{\mathrm{r}=2}^{16} \mathrm{Vu}_{\mathrm{r}} \\
& -2 \mathrm{k}_{\mathrm{A}-\mathrm{A}}\left[\mathrm{A}_{1}\right] \sum_{\mathrm{r}=1}^{13} \mathrm{~A}_{\mathrm{r}}+\mathrm{k}_{\mathrm{E}-\mathrm{E}}\left[\mathrm{E}_{0}{ }_{0}\right]\left[\mathrm{E} *_{1}\right]
\end{aligned}
$$

The term $\mathrm{k}_{\mathrm{DESULF}}\left[\mathrm{A}_{0}\right] \sum_{\mathrm{r}=2}^{16} \mathrm{Vu}_{\mathrm{r}}$ arises due to formation of $\mathrm{A}_{1}$ via the desulfuration reactions that occur in the reversion stage of the vulcanization. Likewise, the PBEs for sulfurating species with other polysulfidic lengths are given below:

For $2 \leqq x \leqq 6$

$$
\begin{aligned}
\frac{\mathrm{d}}{\mathrm{dt}}\left[\mathrm{A}_{\mathrm{x}}\right]= & -2 \mathrm{k}_{\mathrm{A}-\mathrm{R}}\left[\mathrm{A}_{\mathrm{x}}\right]-\mathrm{k}_{\mathrm{A}-\mathrm{S}}\left[\mathrm{A}_{\mathrm{x}}\right]\left[\mathrm{S}_{8}\right]+2 \mathrm{k}_{\mathrm{A}-\mathrm{A}}\left[\mathrm{A}_{0}\right] \sum_{\mathrm{r}=\mathrm{x}+1}^{14} \mathrm{~A}_{\mathrm{r}}-(\mathrm{x}-1) \mathrm{k}_{\mathrm{A}-\mathrm{A}}\left[\mathrm{A}_{0}\right]\left[\mathrm{A}_{\mathrm{x}}\right] \\
& -2 \mathrm{k}_{\mathrm{A}-\mathrm{A}}\left[\mathrm{A}_{\mathrm{x}}\right] \sum_{\mathrm{r}=1}^{14-\mathrm{x}} \mathrm{A}_{\mathrm{r}}+\mathrm{k}_{\mathrm{E}-\mathrm{E}}\left[\mathrm{E}_{0}^{*}\right]\left[\mathrm{E}_{\mathrm{x}}^{*}\right]+\mathrm{k}_{\mathrm{A}-\mathrm{A}} \sum_{\mathrm{r}=1}^{\mathrm{x}-1}\left[\mathrm{~A}_{\mathrm{r}}\right]\left[\mathrm{A}_{\mathrm{x}-\mathrm{r}}\right]
\end{aligned}
$$

For $x=7$

$$
\begin{aligned}
\frac{\mathrm{d}}{\mathrm{dt}}\left[\mathrm{A}_{7}\right]= & -2 \mathrm{k}_{\mathrm{A}-\mathrm{R}}\left[\mathrm{A}_{7}\right]+2 \mathrm{k}_{\mathrm{A}-\mathrm{A}}\left[\mathrm{A}_{0}\right] \sum_{\mathrm{r}=8}^{14} \mathrm{~A}_{\mathrm{r}}-6 \mathrm{k}_{\mathrm{A}-\mathrm{A}}\left[\mathrm{A}_{0}\right]\left[\mathrm{A}_{7}\right]-2 \mathrm{k}_{\mathrm{A}-\mathrm{A}}\left[\mathrm{A}_{7}\right] \sum_{\mathrm{r}=1}^{7} \mathrm{~A}_{\mathrm{r}} \\
& +\mathrm{k}_{\mathrm{E}-\mathrm{E}}\left[\mathrm{E}_{0}^{*}{ }_{0}\right]\left[\mathrm{E}_{7}{ }_{7}\right]+\mathrm{k}_{\mathrm{A}-\mathrm{A}} \sum_{\mathrm{r}=1}^{6}\left[\mathrm{~A}_{\mathrm{r}}\right]\left[\mathrm{A}_{7-\mathrm{r}}\right]
\end{aligned}
$$

For $8 \leq x \leq 14$

$$
\begin{aligned}
\frac{\mathrm{d}}{\mathrm{dt}}\left[\mathrm{A}_{\mathrm{x}}\right]= & -2 \mathrm{k}_{\mathrm{A}-\mathrm{R}}\left[\mathrm{A}_{\mathrm{x}}\right]+\mathrm{k}_{\mathrm{A}-\mathrm{S}}\left[\mathrm{A}_{\mathrm{x}-8}\right]\left[\mathrm{S}_{8}\right]+2 \mathrm{k}_{\mathrm{A}-\mathrm{A}}\left[\mathrm{A}_{0}\right] \sum_{\mathrm{r}=\mathrm{x}+1}^{14} \mathrm{~A}_{\mathrm{r}}-(\mathrm{x}-1) \mathrm{k}_{\mathrm{A}-\mathrm{A}}\left[\mathrm{A}_{0}\right]\left[\mathrm{A}_{\mathrm{x}}\right] \\
& -2 \mathrm{k}_{\mathrm{A}-\mathrm{A}}\left[\mathrm{A}_{\mathrm{x}}\right] \sum_{\mathrm{r}=1}^{14-\mathrm{x}} \mathrm{A}_{\mathrm{r}}+\mathrm{k}_{\mathrm{E}-\mathrm{E}}\left[\mathrm{E}_{0}^{*}\right]\left[\mathrm{E}_{\mathrm{x}}^{*}\right]+\mathrm{k}_{\mathrm{A}-\mathrm{A}} \sum_{\mathrm{r}=1}^{\mathrm{x}-1}\left[\mathrm{~A}_{\mathrm{r}}\right]\left[\mathrm{A}_{\mathrm{x}-\mathrm{r}}\right]
\end{aligned}
$$

The source of differences between Equations. 8, 9 and 10 is the truncation of the length of the sulfurating species at $\mathrm{A}_{14}$ which follows from Assumption 5 that restricts the maximum crosslink to be of length 16. Although the truncation results in a less elegant representation, numerical considerations require that the PBEs be truncated at some point, which results in these different forms for the $\mathrm{A}_{\mathrm{x}}$ PBEs. 
The PBE approach will now be developed for sulfur. Sulfur can be picked-up by $\mathrm{A}_{\mathrm{x}}$ via R.21, by $\mathrm{E}_{\mathrm{x}}^{*}$ via $\mathrm{R} .18$ and by $\mathrm{B}_{\mathrm{x}}{ }_{\mathrm{x}}$ via $\mathrm{R} .50$. The restriction of maximum length of polysulfidics to 16 sulfur atoms, restricts that $A_{x}$ up to only $A_{6}$ can pick-up $S_{8}, B^{*}{ }_{x}$ up to only $B_{8}^{*}$ pick-up $S_{8}$ and $E^{*}{ }_{x}$ up to only $\mathrm{E}_{8}^{*}$ can pick-up $\mathrm{S}_{8}$. The balance equation for sulfur is:

$$
\frac{\mathrm{d}}{\mathrm{dt}}\left[\mathrm{S}_{8}\right]=-\mathrm{k}_{\mathrm{A}-\mathrm{S}}\left[\mathrm{A}_{0}\right]\left[\mathrm{S}_{8}\right]-\mathrm{k}_{\mathrm{E}-\mathrm{S}}\left[\mathrm{S}_{8}\right] \sum_{\mathrm{r}=0}^{8} \mathrm{E}_{\mathrm{r}}^{*}-\mathrm{k}_{\mathrm{BST}-\mathrm{S}}\left[\mathrm{S}_{8}\right] \sum_{\mathrm{r}=1}^{8} \mathrm{~B}^{*}{ }_{\mathrm{r}}
$$

The PBEs for the crosslink precursors, $\mathrm{B}_{\mathrm{x}}$ will now be developed. $\mathrm{B}_{0}$ is an inactivated or dead species, since it does not fragment further because of the stability of the C-S bond. However, all other lengths of $B_{x}$ are active crosslink precursors. $B_{x}$ is formed from the sulfurating species $A_{x}$ through R.34 and by the addition of benzothiazole terminated polysulfidic radicals $\mathrm{E}_{\mathrm{x}}^{*}$ to an allylic position in the elastomer via R.49. Radical quenching reactions as given by R.53 between $\mathrm{A}_{\mathrm{x}}$ and $\mathrm{B}_{\mathrm{y}}^{*}$ also contribute to $\mathrm{B}_{\mathrm{x}}$ formation. $\mathrm{B}_{\mathrm{x}}$ species are consumed via R.42, where $\mathrm{B}_{\mathrm{x}}$ is split into a persulfenyl radical $\mathrm{B}_{\mathrm{x}}{ }_{\mathrm{x}}$ and a benzothiazole radical $\mathrm{E}_{\mathrm{x}}{ }_{\mathrm{x}}$. The overall balance for $\mathrm{B}_{0}$ and $\mathrm{B}_{\mathrm{x}}$ are given in the following equations:

$$
\frac{\mathrm{dB}_{0}}{\mathrm{dt}}=\mathrm{k}_{\mathrm{A}-\mathrm{R}}\left[\mathrm{A}_{0}\right]+\mathrm{k}_{\mathrm{E}-\mathrm{R}}\left[\mathrm{E}_{0}{ }_{0}\right]
$$

For $1 \leq x \leq 14$

$$
\frac{\mathrm{d}}{\mathrm{dt}}\left[\mathrm{B}_{\mathrm{x}}\right]=2 \mathrm{k}_{\mathrm{A}-\mathrm{R}}\left[\mathrm{A}_{\mathrm{x}}\right]-\mathrm{xk}_{\mathrm{B}-\mathrm{R}}\left[\mathrm{B}_{\mathrm{x}}\right]+\mathrm{k}_{\mathrm{E}-\mathrm{R}}\left[\mathrm{E}^{*}{ }_{\mathrm{x}}\right]+\mathrm{k}_{\mathrm{A}-\mathrm{BST}}\left[\mathrm{A}_{0}\right]\left[\mathrm{B}{ }_{\mathrm{x}}\right]
$$

For $x=15$ and 16

$$
\frac{\mathrm{d}}{\mathrm{dt}}\left[\mathrm{B}_{\mathrm{x}}\right]=-x \mathrm{k}_{\mathrm{B}-\mathrm{R}}\left[\mathrm{B}_{\mathrm{x}}\right]+\mathrm{k}_{\mathrm{E}-\mathrm{R}}\left[\mathrm{E} *_{\mathrm{x}}\right]+\mathrm{k}_{\mathrm{A}-\mathrm{BST}}\left[\mathrm{A}_{0}\right]\left[\mathrm{B} *_{\mathrm{x}}\right]
$$

Here again the three different forms of the PBE are a consequence of Assumption 5 limiting the length of the polysulfidic chains to 16 . The crosslink precursors $B_{x}$ lead to the formation of persulfenyl radicals, $\mathrm{B}_{\mathrm{x}}{ }_{\mathrm{x}}$ via $\mathrm{R} .42$, where $\mathrm{B}_{\mathrm{x}}{ }_{\mathrm{x}}$ subsequently reacts with an allylic position in a neighboring isoprene chain to form a crosslink by R.43 or reacts with an allylic hydrogen in the same isoprene chain to form a cyclic sulfide or loop via R.52. Since $\mathrm{B}_{\mathrm{x}}{ }_{\mathrm{x}}$ is an active polysulfidic radical, it also participates in the sulfur pick-up reactions via R.50 and the radical quenching reactions with the sulfurating agents defined in R.53. The overall balance for the $\mathrm{B}_{\mathrm{x}}{ }_{\mathrm{x}}$ species is given by:

\section{For $1 \leq x \leq 8$}

$$
\frac{\mathrm{d}}{\mathrm{dt}}\left[\mathrm{B} *_{\mathrm{x}}\right]=\mathrm{k}_{\mathrm{B}-\mathrm{R}} \sum_{\mathrm{r}=\mathrm{x}_{\mathrm{x}}}^{16}\left[\mathrm{~B}_{\mathrm{r}}\right]-\left(\mathrm{k}_{\mathrm{VU}}+\mathrm{k}_{\mathrm{LOOP}}\right)\left[\mathrm{B} *_{\mathrm{x}}\right]-\mathrm{k}_{\mathrm{BST}-\mathrm{S}}\left[\mathrm{B} *_{\mathrm{x}}\right]\left[\mathrm{S}_{8}\right]-\mathrm{k}_{\mathrm{A}-\mathrm{BST}}\left[\mathrm{A}_{0}\right]\left[\mathrm{B} *_{\mathrm{x}}\right]
$$


For $9 \leq x \leq 16$

$$
\frac{\mathrm{d}}{\mathrm{dt}}\left[\mathrm{B} *_{\mathrm{x}}\right]=\mathrm{k}_{\mathrm{B}-\mathrm{R}} \sum_{\mathrm{r}=\mathrm{x}}^{16}\left[\mathrm{~B}_{\mathrm{r}}\right]-\left(\mathrm{k}_{\mathrm{VU}}+\mathrm{k}_{\mathrm{LOOP}}\right)\left[\mathrm{B}{ }_{\mathrm{x}}\right]+\mathrm{k}_{\mathrm{BST}-\mathrm{S}}\left[\mathrm{B} *_{\mathrm{x}-8}\right]\left[\mathrm{S}_{8}\right]-\mathrm{k}_{\mathrm{A}-\mathrm{BST}}\left[\mathrm{A}_{0}\right]\left[\mathrm{B} *_{\mathrm{x}}\right]
$$

The $\mathrm{B}_{\mathrm{x}}^{*}$ species can lead to the formation of mono, di and polysulfidic crosslinks via R.43. As discussed in Section III.C, the crosslinks are further involved in desulfuration reactions via R.60 and 61 and through degradation reactions given by R.62 and 63 . While desulfuration involves the removal of sulfur from polysulfidic crosslinks by $(\mathrm{BtS})_{2} \mathrm{Zn}$ or BtS-SBt and is captured through the rate constant $\mathrm{k}_{\mathrm{DESULF}}$, the degradation reaction is captured in a lumped sense through the rate constant $\mathrm{k}_{\mathrm{DEG}}$. Specifically, the degradation reaction is given by:

$$
\text { Rubber-S } \mathrm{x}_{\mathrm{x}} \text {-Rubber } \stackrel{\mathrm{k}_{\mathrm{DEG}}}{\longrightarrow} \text { Dead End }
$$

where the dead-end includes terminated thiols, conjugated dienes and trienes and other mainchain modifications. It is useful to recall here that the monosulfidic crosslinks are relatively immune to these thermal degradative reactions because of the high strength of the monosulfidic crosslinks. However, polysulfidic crosslinks are thermally labile and may degrade into dead ends. The differential balance on monosulfides is therefore:

$$
\frac{\mathrm{d}}{\mathrm{dt}}\left[\mathrm{Vu}_{1}\right]=\mathrm{k}_{\mathrm{VU}}\left[\mathrm{B} *_{1}\right]+\mathrm{k}_{\mathrm{DESULF}}\left[\mathrm{A}_{0}\right]\left[\mathrm{Vu}_{2}\right]
$$

whereas for polysulfides, the PBE is:

For $2 \leq x \leq 16$

$$
\frac{\mathrm{d}}{\mathrm{dt}}\left[\mathrm{Vu}_{\mathrm{x}}\right]=\mathrm{k}_{\mathrm{VU}}\left[\mathrm{B}{ }_{\mathrm{x}}\right]-\mathrm{k}_{\mathrm{DEG}}\left[\mathrm{Vu}_{\mathrm{x}}\right]-\mathrm{k}_{\mathrm{DESULF}}\left[\mathrm{A}_{0}\right]\left[\mathrm{Vu}_{\mathrm{x}}\right]+\mathrm{k}_{\mathrm{DESULF}}\left[\mathrm{A}_{0}\right]\left[\mathrm{Vu}_{\mathrm{x}+1}\right]
$$

The last term $\mathrm{k}_{\text {DESULF }}\left[\mathrm{A}_{0}\right]\left[\mathrm{Vu}_{\mathrm{x}+1}\right]$ is absent for the case $\mathrm{x}=16$.

The PBEs for the accelerator-terminated polysulfidic radicals $\mathrm{E}_{\mathrm{x}}^{*}$ will now be developed. These radicals are formed in R.42 by precursor fragmentation and R.53 by persulfenyl radical quenching; and, the radicals are consumed in R.16 by radical recombination, in R.19 by pickingup $\mathrm{S}_{8}$, and in R.49 by reacting with allylic hydrogen in rubber to give crosslink precursors. The PBEs for $E_{x}^{*}$ are:

$\underline{F o r} x=0$

$$
\mathrm{k}_{\mathrm{B}-\mathrm{R}} \sum_{\mathrm{r}=1}^{16} \mathrm{~B}_{\mathrm{r}}+\mathrm{k}_{\mathrm{A}-\mathrm{BST}}\left[\mathrm{A}_{0}\right] \sum_{\mathrm{r}=1}^{16} \mathrm{~B} *_{\mathrm{r}}-\mathrm{k}_{\mathrm{E}-\mathrm{R}}\left[\mathrm{E} *_{0}\right]-\mathrm{k}_{\mathrm{E}-\mathrm{E}}\left[\mathrm{E} *_{0}\right] \sum_{\mathrm{r}=0}^{16} \mathrm{E} *_{\mathrm{r}}-\mathrm{k}_{\mathrm{E}-\mathrm{S}}\left[\mathrm{E} *_{0}\right]\left[\mathrm{S}_{8}\right]
$$

For $1 \leqq x \leqq 7$

$$
\frac{d}{d t}\left[E *_{x}\right]=k_{B-R} \sum_{r=x+1}^{16} B_{r}-k_{E-R}\left[E *_{x}\right]-k_{E-S}\left[E *_{x}\right]\left[S_{8}\right]-k_{E-E}\left[E *_{x}\right]\left[E *_{0}\right]
$$


$\underline{F o r} x=8$

$$
\frac{\mathrm{d}}{\mathrm{dt}}\left[\mathrm{E} *_{8}\right]=\mathrm{k}_{\mathrm{B}-\mathrm{R}} \sum_{\mathrm{r}=9}^{16} \mathrm{~B}_{\mathrm{r}}-\mathrm{k}_{\mathrm{E}-\mathrm{R}}\left[\mathrm{E} *_{8}\right]-\mathrm{k}_{\mathrm{E}-\mathrm{S}}\left[\mathrm{E} *_{8}\right]\left[\mathrm{S}_{8}\right]+\mathrm{k}_{\mathrm{E}-\mathrm{S}}\left[\mathrm{E} *_{0}\right]\left[\mathrm{S}_{8}\right]-\mathrm{k}_{\mathrm{E}-\mathrm{E}}\left[\mathrm{E} *_{8}\right]\left[\mathrm{E} *_{0}\right]
$$

For $9 \leq x \leq 14$

$$
\frac{\mathrm{d}}{\mathrm{dt}}\left[\mathrm{E}{ }_{\mathrm{x}}\right]=\mathrm{k}_{\mathrm{B}-\mathrm{R}} \sum_{\mathrm{r}=\mathrm{x}+1}^{16} \mathrm{~B}_{\mathrm{r}}-\mathrm{k}_{\mathrm{E}-\mathrm{R}}\left[\mathrm{E}{ }_{\mathrm{x}}^{*}\right]+\mathrm{k}_{\mathrm{E}-\mathrm{S}}\left[\mathrm{E}{ }_{\mathrm{x}-8}\right]\left[\mathrm{S}_{8}\right]-\mathrm{k}_{\mathrm{E}-\mathrm{E}}\left[\mathrm{E}{ }_{\mathrm{x}}\right]\left[\mathrm{E}{ }_{0}\right]
$$

\section{$\underline{\text { For } x=15 \text { and } 16}$}

$$
\begin{gathered}
\frac{\mathrm{d}}{\mathrm{dt}}\left[\mathrm{E}^{*}{ }_{15}\right]=\mathrm{k}_{\mathrm{B}-\mathrm{R}}\left[\mathrm{B}_{16}\right]-\mathrm{k}_{\mathrm{E}-\mathrm{R}}\left[\mathrm{E} *_{15}\right]+\mathrm{k}_{\mathrm{E}-\mathrm{S}}\left[\mathrm{E}_{7}{ }_{7}\right]\left[\mathrm{S}_{8}\right] \\
\frac{\mathrm{d}}{\mathrm{dt}}\left[\mathrm{E}{ }_{16}\right]=-\mathrm{k}_{\mathrm{B}-\mathrm{R}}\left[\mathrm{E}{ }_{16}\right]+\mathrm{k}_{\mathrm{E}-\mathrm{S}}\left[\mathrm{E}_{8}{ }_{8}\right]\left[\mathrm{S}_{8}\right]
\end{gathered}
$$

Here again the reason that there are five different forms for the PBEs for $\mathrm{E}_{\mathrm{x}}{ }_{\mathrm{x}}$ is because the length of the polysulfidic chain has been terminated at $x=16$. Finally, we complete the PBEs by performing rate-balances on MBT and the Dead Ends; specifically,

$$
\begin{gathered}
\frac{\mathrm{d}}{\mathrm{dt}}[\mathrm{MBT}]=\mathrm{k}_{\mathrm{MBS}}[\mathrm{MBS}]-\mathrm{k}_{\mathrm{MBS}-\mathrm{MBT}}[\mathrm{MBT}][\mathrm{MBS}]+\mathrm{k}_{\mathrm{A}-\mathrm{R}}\left[\mathrm{A}_{0}\right]+2 \mathrm{k}_{\mathrm{A}-\mathrm{R}} \sum_{\mathrm{r}=1}^{14} \mathrm{~A}_{\mathrm{r}} \\
\frac{\mathrm{d}}{\mathrm{dt}}[\text { Dead }- \text { Ends }]=\mathrm{k}_{\text {DEG }} \sum_{\mathrm{r}=1}^{16} \mathrm{Vu}_{\mathrm{r}}+\mathrm{k}_{\text {LOOP }} \sum_{\mathrm{r}=1}^{16} \mathrm{~B}_{\mathrm{r}}{ }_{\mathrm{r}}
\end{gathered}
$$

Equations 5 through 26 form the complete set of PBEs for Model-I, which when expanded for all the individual lengths constitutes 100 nonlinear, coupled ordinary differential equations. The PBEs are for the following species: MBS, $A_{x}, S_{8}, B_{x}, B^{*}{ }_{x}, E_{x}^{*}$, MBT and Dead Ends for $\mathrm{x} \leq$ 16 , and involve the rate constants: $\mathrm{k}_{\mathrm{MBS}}, \mathrm{k}_{\mathrm{MBS}-\mathrm{MBT}}, \mathrm{k}_{\mathrm{A}-\mathrm{S}}, \mathrm{k}_{\mathrm{A}-\mathrm{A}}, \mathrm{k}_{\mathrm{A}-\mathrm{R}}, \mathrm{k}_{\mathrm{B}-\mathrm{R}}, \mathrm{k}_{\mathrm{VU}}, \mathrm{k}_{\mathrm{DEG}}, \mathrm{k}_{\mathrm{DESULF}}, \mathrm{k}_{\mathrm{E}-}$ $\mathrm{S}, \mathrm{k}_{\mathrm{E}-\mathrm{R}}, \mathrm{k}_{\mathrm{BST}-\mathrm{S}}, \mathrm{k}_{\mathrm{LOOP}}, \mathrm{k}_{\mathrm{A}-\mathrm{BST}}$ and $\mathrm{k}_{\mathrm{E}-\mathrm{E}}$. For this set of differential equations, the initial conditions are fully specified by the initial concentrations of accelerator $[M B S]_{t=0}$ and sulfur $\left[\mathrm{S}_{8}\right]_{\mathrm{t}=0}$ in the formulation, where the concentration of all other species is zero. We believe that the most reasonable description of the vulcanization kinetics for benzothiazole sulfenamide accelerated systems, assuming sulfur is picked-up as $S_{8}$, is given by these 100 nonlinear coupled ordinary differential equations with 15 rate constants. The solution of these PBEs along with the determination of the rate constants will be discussed in Section VII.

\section{B. POPULATION BALANCE EQUATIONS FOR MODEL-II: SEQUENTIAL SULFUR PICK-UP}

In Section IV.A PBEs were developed for Model-I, where it was assumed that the various species capable of sulfur activation, i.e. $\mathrm{A}_{\mathrm{x}}, \mathrm{E}_{\mathrm{x}}^{*}$ and $\mathrm{B}_{\mathrm{x}}{ }_{\mathrm{x}}$ all pick-up sulfur as $\mathrm{S}_{8}$ to respectively 
form $\mathrm{A}_{\mathrm{x}+8}, \mathrm{E}_{\mathrm{x}+8}^{*}$ and $\mathrm{B}_{\mathrm{x}+8}^{*}$. We will now develop the PBEs for Model-II, where it will be assumed that sulfur activation occurs via a single sulfur atom at a time as given in R.23. The reaction scheme of Model-II is summarized in Table VI. Since the derivation of the PBEs for Model-II is nearly identical to that already described for Model-I in Section IV.A, we will omit a detailed discussion of the derivation and only present the final PBEs. The PBEs for the sulfurating species, $\mathrm{A}_{\mathrm{x}}$ are:

$$
\begin{aligned}
& \frac{\mathrm{d}}{\mathrm{dt}}\left[\mathrm{A}_{0}\right]=\mathrm{k}_{\mathrm{MBS}-\mathrm{MBT}}[\mathrm{MBT}][\mathrm{MBS}]-\mathrm{k}_{\mathrm{A}-\mathrm{A}}\left[\mathrm{A}_{0}\right] \sum_{\mathrm{r}=2}^{14}(\mathrm{r}-1) \mathrm{A}_{\mathrm{r}}-\mathrm{k}_{\mathrm{A}-\mathrm{S}}\left[\mathrm{A}_{0}\right] \sum_{\mathrm{y}=1}^{8}\left[\mathrm{~S}_{\mathrm{y}}\right] \\
& -\mathrm{k}_{\mathrm{A}-\mathrm{R}}\left[\mathrm{A}_{0}\right]-\mathrm{k}_{\mathrm{A}-\mathrm{BST}}\left[\mathrm{A}_{0}\right] \sum_{\mathrm{r}=1}^{16} \mathrm{~B} *_{\mathrm{r}}+\frac{1}{2} \mathrm{k}_{\mathrm{E}-\mathrm{E}}\left[\mathrm{E}_{0}^{*}\right]^{2}-\mathrm{k}_{\text {DESULF }}\left[\mathrm{A}_{0}\right] \sum_{\mathrm{r}=2}^{16} \mathrm{Vu}_{\mathrm{r}} \\
& +\mathrm{k}_{\mathrm{A}-\mathrm{A}} \sum_{\mathrm{x}=1}^{13} \sum_{\mathrm{y}=1}^{13-\mathrm{x}} \mathrm{A}_{\mathrm{x}} \mathrm{A}_{\mathrm{y}} \\
& \frac{\mathrm{d}}{\mathrm{dt}}\left[\mathrm{A}_{1}\right]=-2 \mathrm{k}_{\mathrm{A}-\mathrm{R}}\left[\mathrm{A}_{1}\right]-\mathrm{k}_{\mathrm{A}-\mathrm{S}}\left(\left[\mathrm{A}_{1}\right]-\left[\mathrm{A}_{0}\right]\right) \sum_{\mathrm{y}=1}^{8}\left[\mathrm{~S}_{\mathrm{y}}\right]+2 \mathrm{k}_{\mathrm{A}-\mathrm{A}}\left[\mathrm{A}_{0}\right] \sum_{\mathrm{r}=2}^{14} \mathrm{~A}_{\mathrm{r}}+\mathrm{k}_{\text {DESULF }}\left[\mathrm{A}_{0}\right] \sum_{\mathrm{r}=2}^{16} \mathrm{Vu}_{\mathrm{r}} \\
& -2 \mathrm{k}_{\mathrm{A}-\mathrm{A}}\left[\mathrm{A}_{1}\right] \sum_{\mathrm{r}=1}^{13} \mathrm{~A}_{\mathrm{r}}+\mathrm{k}_{\mathrm{E}-\mathrm{E}}\left[\mathrm{E} *_{0}\right]\left[\mathrm{E} *_{1}\right]
\end{aligned}
$$

$\underline{\text { For } 2 \leq x \leq 13}$

$$
\begin{aligned}
\frac{\mathrm{d}}{\mathrm{dt}}\left[\mathrm{A}_{\mathrm{x}}\right]= & -2 \mathrm{k}_{\mathrm{A}-\mathrm{R}}\left[\mathrm{A}_{\mathrm{x}}\right]-\mathrm{k}_{\mathrm{A}-\mathrm{S}}\left(\left[\mathrm{A}_{\mathrm{x}}\right]-\left[\mathrm{A}_{\mathrm{x}-1}\right]\right) \sum_{\mathrm{y}=1}^{8}\left[\mathrm{~S}_{\mathrm{y}}\right]+2 \mathrm{k}_{\mathrm{A}-\mathrm{A}}\left[\mathrm{A}_{0}\right] \sum_{\mathrm{r}=\mathrm{x}+1}^{14} \mathrm{~A}_{\mathrm{r}} \\
& -(\mathrm{x}-1) \mathrm{k}_{\mathrm{A}-\mathrm{A}}\left[\mathrm{A}_{0}\right]\left[\mathrm{A}_{\mathrm{x}}\right]-2 \mathrm{k}_{\mathrm{A}-\mathrm{A}}\left[\mathrm{A}_{\mathrm{x}}\right] \sum_{\mathrm{r}=1}^{14-\mathrm{x}} \mathrm{A}_{\mathrm{r}}+\mathrm{k}_{\mathrm{E}-\mathrm{E}}\left[\mathrm{E}_{0}^{*}\right]\left[\mathrm{E}_{\mathrm{x}}^{*}\right] \\
& +\mathrm{k}_{\mathrm{A}-\mathrm{A}} \sum_{\mathrm{r}=1}^{\mathrm{x}-1}\left[\mathrm{~A}_{\mathrm{r}}\right]\left[\mathrm{A}_{\mathrm{x}-\mathrm{r}}\right]
\end{aligned}
$$

For $x=14$

$$
\begin{aligned}
\frac{\mathrm{d}}{\mathrm{dt}}\left[\mathrm{A}_{14}\right]= & -2 \mathrm{k}_{\mathrm{A}-\mathrm{R}}\left[\mathrm{A}_{14}\right]+\mathrm{k}_{\mathrm{A}-\mathrm{S}}\left[\mathrm{A}_{13}\right] \sum_{\mathrm{y}=1}^{8}\left[\mathrm{~S}_{\mathrm{y}}\right]-13 \mathrm{k}_{\mathrm{A}-\mathrm{A}}\left[\mathrm{A}_{0}\right]\left[\mathrm{A}_{14}\right]+\mathrm{k}_{\mathrm{E}-\mathrm{E}}\left[\mathrm{E} *_{0}\right]\left[\mathrm{E}{ }_{14}\right] \\
& +\mathrm{k}_{\mathrm{A}-\mathrm{A}} \sum_{\mathrm{r}=1}^{13}\left[\mathrm{~A}_{\mathrm{r}}\right]\left[\mathrm{A}_{14-\mathrm{r}}\right]
\end{aligned}
$$

Since the longest $A_{x}$ considered is $A_{14}$, the sulfur pick-up reactions for $A_{x}$ where $x$ greater than 14 would not be consistent with Assumption 5. The PBEs for $B_{x}$ remain the same as those of Model-I as given in Equations 12, 13 and 14. The new PBEs for species $\mathrm{B}^{*}{ }_{\mathrm{x}}$ are:

$$
\frac{\mathrm{d}}{\mathrm{dt}}\left[\mathrm{B} *_{1}\right]=\mathrm{k}_{\mathrm{B}-\mathrm{R}} \sum_{\mathrm{r}=1}^{16}\left[\mathrm{~B}_{\mathrm{r}}\right]-\left(\mathrm{k}_{\mathrm{VU}}+\mathrm{k}_{\mathrm{LOOP}}\right)\left[\mathrm{B} *_{1}\right]-\mathrm{k}_{\mathrm{BST}-\mathrm{S}}\left[\mathrm{B} *_{1}\right] \sum_{\mathrm{y}=1}^{8}\left[\mathrm{~S}_{\mathrm{y}}\right]-\mathrm{k}_{\mathrm{A}-\mathrm{BST}}\left[\mathrm{A}_{0}\right]\left[\mathrm{B} *_{1}\right]
$$


$\underline{\text { For } 2 \leq x \leq 15}$

$\frac{\mathrm{d}}{\mathrm{dt}}\left[\mathrm{B}{ }_{\mathrm{x}}\right]=\mathrm{k}_{\mathrm{B}-\mathrm{R}} \sum_{\mathrm{r}=\mathrm{x}}^{16}\left[\mathrm{~B}_{\mathrm{r}}\right]-\left(\mathrm{k}_{\mathrm{VU}}+\mathrm{k}_{\mathrm{LOOP}}\right)\left[\mathrm{B}{ }^{*}{ }_{\mathrm{x}}\right]-\mathrm{k}_{\mathrm{BST}-\mathrm{S}}\left(\left[\mathrm{B}{ }_{\mathrm{x}}\right]-\left[\mathrm{B}{ }_{\mathrm{x}-1}\right]\right) \sum_{\mathrm{y}=1}^{8}\left[\mathrm{~S}_{\mathrm{y}}\right]-\mathrm{k}_{\mathrm{A}-\mathrm{BST}}\left[\mathrm{A}_{0}\right]\left[\mathrm{B}{ }_{\mathrm{x}}\right]$

\section{$\underline{\text { For } x=16}$}

$$
\frac{\mathrm{d}}{\mathrm{dt}}\left[\mathrm{B} *{ }_{16}\right]=\mathrm{k}_{\mathrm{B}-\mathrm{R}}\left[\mathrm{B}_{16}\right]-\left(\mathrm{k}_{\mathrm{VU}}+\mathrm{k}_{\mathrm{LOOP}}\right)\left[\mathrm{B}{ }_{16}\right]+\mathrm{k}_{\mathrm{BST}-\mathrm{S}}\left[\mathrm{B} *{ }_{15}\right] \sum_{\mathrm{y}=1}^{8}\left[\mathrm{~S}_{\mathrm{y}}\right]-\mathrm{k}_{\mathrm{A}-\mathrm{BST}}\left[\mathrm{A}_{0}\right]\left[\mathrm{B} *{ }_{16}\right]
$$

The balance equations for the $\mathrm{E}_{\mathrm{x}}{ }_{\mathrm{x}}$ species are:

$$
\begin{aligned}
\frac{\mathrm{d}}{\mathrm{dt}}\left[\mathrm{E} *_{0}\right] & =\mathrm{k}_{\mathrm{B}-\mathrm{R}} \sum_{\mathrm{r}=1}^{16} \mathrm{~B}_{\mathrm{r}}-\mathrm{k}_{\mathrm{E}-\mathrm{R}}\left[\mathrm{E} *_{0}\right]-\mathrm{k}_{\mathrm{E}-\mathrm{E}}\left[\mathrm{E} *_{0}\right] \sum_{\mathrm{r}=0}^{16}\left[\mathrm{E} *_{\mathrm{r}}\right]-\mathrm{k}_{\mathrm{E}-\mathrm{S}}\left[\mathrm{E} *_{0}\right] \sum_{\mathrm{y}=1}^{8}\left[\mathrm{~S}_{\mathrm{y}}\right] \\
& +\mathrm{k}_{\mathrm{A}-\mathrm{BST}}\left[\mathrm{A}_{0}\right] \sum_{\mathrm{r}=1}^{16} \mathrm{~B}{ }_{\mathrm{r}}
\end{aligned}
$$

For $1 \leq x \leq 14$

$$
\begin{gathered}
\frac{\mathrm{d}}{\mathrm{dt}}\left[\mathrm{E} *_{\mathrm{x}}\right]=\mathrm{k}_{\mathrm{B}-\mathrm{R}} \sum_{\mathrm{r}=\mathrm{x}+1}^{16} \mathrm{~B}_{\mathrm{r}}-\mathrm{k}_{\mathrm{E}-\mathrm{R}}\left[\mathrm{E} *_{\mathrm{x}}\right]-\mathrm{k}_{\mathrm{E}-\mathrm{S}}\left(\left[\mathrm{E} *_{\mathrm{x}}\right]-\left[\mathrm{E} *_{\mathrm{x}-1}\right]\right) \sum_{\mathrm{y}=1}^{8}\left[\mathrm{~S}_{\mathrm{y}}\right]-\mathrm{k}_{\mathrm{E}-\mathrm{E}}\left[\mathrm{E} *_{\mathrm{x}}\right]\left[\mathrm{E} *_{0}\right] \\
\frac{\mathrm{d}}{\mathrm{dt}}\left[\mathrm{E}{ }^{*}{ }_{15}\right]=\mathrm{k}_{\mathrm{B}-\mathrm{R}}\left[\mathrm{B}_{16}\right]-\mathrm{k}_{\mathrm{E}-\mathrm{R}}\left[\mathrm{E}{ }^{*}{ }_{15}\right]-\mathrm{k}_{\mathrm{E}-\mathrm{S}}\left(\left[\mathrm{E}^{*}{ }_{15}\right]-\left[\mathrm{E}{ }_{14}\right]\right) \sum_{\mathrm{y}=1}^{8}\left[\mathrm{~S}_{\mathrm{y}}\right] \\
\frac{\mathrm{d}}{\mathrm{dt}}\left[\mathrm{E} *{ }_{16}\right]=-\mathrm{k}_{\mathrm{E}-\mathrm{R}}\left[\mathrm{E} *_{16}\right]+\mathrm{k}_{\mathrm{E}-\mathrm{S}}\left[\mathrm{E} *{ }_{15}\right] \sum_{\mathrm{y}=1}^{8}\left[\mathrm{~S}_{\mathrm{y}}\right]
\end{gathered}
$$

And finally, the PBEs for sulfur are:

For $1 \leqq x \leqq 7$

$\frac{d}{d t}\left[S_{x}\right]=-k_{A-S} \sum_{r=0}^{13}\left[A_{r}\right]\left(S_{x}-S_{x+1}\right)-k_{E-S} \sum_{r=0}^{15}\left[E_{r}^{*}\right]\left(S_{x}-S_{x+1}\right)-k_{B S T-S} \sum_{r=1}^{15}\left[B *_{r}\right]\left(S_{x}-S_{x+1}\right)$

For $x=8$

$$
\frac{\mathrm{d}}{\mathrm{dt}}\left[\mathrm{S}_{8}\right]=-\mathrm{k}_{\mathrm{A}-\mathrm{S}} \sum_{\mathrm{r}=0}^{13}\left[\mathrm{~A}_{\mathrm{r}}\right]\left[\mathrm{S}_{8}\right]-\mathrm{k}_{\mathrm{E}-\mathrm{S}} \sum_{\mathrm{r}=0}^{15}\left[\mathrm{E}{ }_{\mathrm{r}}\right]\left[\mathrm{S}_{8}\right]-\mathrm{k}_{\text {BST }-\mathrm{S}} \sum_{\mathrm{r}=1}^{15}\left[\mathrm{~B}{ }_{\mathrm{r}}\right]\left[\mathrm{S}_{8}\right]
$$


The PBEs for the remaining species $\mathrm{Vu}_{\mathrm{x}}, \mathrm{MBT}$ and Dead Ends are the same as in Model-I and are given by Equations 17, 18, 25 and 26, respectively. The initial conditions are the same as that in Model-I. It will be assumed that all the sulfur is initially present as $\mathrm{S}_{8}$ ; thus, $\left[\mathrm{S}_{\mathrm{x}}\right]_{\mathrm{t}=0}=0$ for $1 \leq \mathrm{x} \leq 7$ and $\left[\mathrm{S}_{8}\right]_{\mathrm{t}=0}$ is equal to the initial sulfur concentration expressed as moles of sulfur/8, since there are 8 moles of $S$ in $S_{8}$. For Model-II, where $x \leq 16$, there are a total of 107 nonlinear-coupled ordinary differential equations with 15 rate constants. The solution of the PBEs for Model-II and the determination of these rate constants will be presented in Section VII.

\section{INCORPORATION OF RETARDER IN THE POPULATION BALANCE EQUATIONS}

The incorporation of retarders like CTP into the population balance model does not affect the basic structure of the equations, except for some minor modifications in the rate-equations for MBTS and MBT. The specific equations that are modified are Equations 6 and 25, which are now respectively

$$
\begin{aligned}
\frac{\mathrm{d}}{\mathrm{dt}}\left[\mathrm{A}_{0}\right] & =\mathrm{k}_{\mathrm{MBS}-\mathrm{MBT}}[\mathrm{MBT}][\mathrm{MBS}]-\mathrm{k}_{\mathrm{A}-\mathrm{A}}\left[\mathrm{A}_{0}\right] \sum_{\mathrm{r}=2}^{14}(\mathrm{r}-1) \mathrm{A}_{\mathrm{r}}-\mathrm{k}_{\mathrm{A}-\mathrm{S}}\left[\mathrm{A}_{0}\right]\left[\mathrm{S}_{8}\right] \\
& -\mathrm{k}_{\mathrm{A}-\mathrm{R}}\left[\mathrm{A}_{0}\right]-\mathrm{k}_{\mathrm{A}-\mathrm{BST}}\left[\mathrm{A}_{0}\right] \sum_{\mathrm{r}=1}^{16} \mathrm{~B}_{\mathrm{r}}^{*}+\frac{1}{2} \mathrm{k}_{\mathrm{E}-\mathrm{E}}\left[\mathrm{E}_{0}^{*}\right]_{0}^{2}-\mathrm{k}_{\text {DESULF }}\left[\mathrm{A}_{0}\right] \sum_{\mathrm{r}=2}^{16} \mathrm{Vu}_{\mathrm{r}} \\
& +\mathrm{k}_{\mathrm{A}-\mathrm{A}} \sum_{\mathrm{x}=1 \mathrm{y}=1}^{13} \sum_{\mathrm{y}=1}^{13-\mathrm{x}} \mathrm{A}_{\mathrm{x}} \mathrm{A}_{\mathrm{y}}+\underline{\underline{\mathrm{k}_{\mathrm{CDB}}[\mathrm{MBT}][\mathrm{CDB}]}}
\end{aligned}
$$

and

$$
\begin{aligned}
\frac{\mathrm{d}}{\mathrm{dt}}[\mathrm{MBT}] & =\mathrm{k}_{\mathrm{MBS}}[\mathrm{MBS}]-\mathrm{k}_{\mathrm{MBS}-\mathrm{MBT}}[\mathrm{MBT}][\mathrm{MBS}]+\mathrm{k}_{\mathrm{A}-\mathrm{R}}\left[\mathrm{A}_{0}\right]+2 \mathrm{k}_{\mathrm{A}-\mathrm{R}} \sum_{\mathrm{r}=1}^{14} \mathrm{~A}_{\mathrm{r}} \\
& =\mathrm{-k}_{\mathrm{RET}}[\mathrm{MBT}][\mathrm{CTP}]-\mathrm{k}_{\mathrm{CDB}}[\mathrm{MBT}][\mathrm{CDB}]
\end{aligned}
$$

The underlined terms are the additional contributions in the PBEs for $\mathrm{A}_{0}$ and MBT that accounts for the effect of retarder due to R.68 and R.70. Additional kinetic equations for CTP and CDB are

$$
\begin{gathered}
\frac{\mathrm{d}}{\mathrm{dt}}[\mathrm{CTP}]=-\mathrm{k}_{\mathrm{RET}}[\mathrm{MBT}][\mathrm{CTP}] \\
\frac{\mathrm{d}}{\mathrm{dt}}[\mathrm{CDB}]=\mathrm{k}_{\mathrm{RET}}[\mathrm{MBT}][\mathrm{CTP}]-\mathrm{k}_{\mathrm{CDB}}[\mathrm{MBT}][\mathrm{CDB}]
\end{gathered}
$$

Equations 40 through 42 along with Equations 6 through 24 and 26 represent the mathematical model for vulcanization systems with retarder using the scheme represented in Model-I. $[\text { CTP }]_{t}$ $=0$ is the initial retarder concentration. For Model-II, the PBEs are Equations 27 through 39 as well as the retarder kinetic equations 40 through 42.

\section{ESTIMATION OF LOOPING PROBABILITIES USING CONFORMATIONAL ANALYSIS}

The formation of crosslinks from persulfenyl radicals is the objective of the vulcanization process. If the persulfenyl radical attaches to an allylic carbon on a neighboring isoprene chain, 
it is a crosslink; however, if the attachment is to the same polymer chain from which it originates, the reaction results in an elastically ineffective cyclic sulfide (or a loop) as shown below:

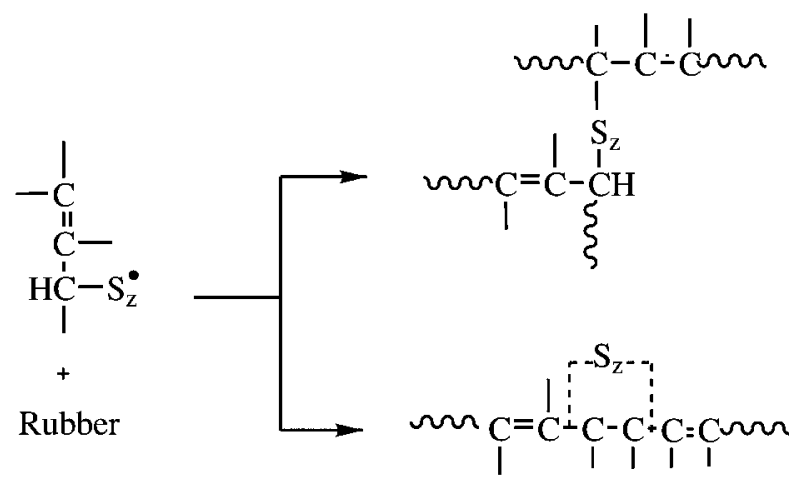

The formation of these cyclic sulfides is important, since they do not contribute to the elastic modulus of the vulcanizate; and therefore, this cyclization reaction is an important source of the modulus reversion observed in the ODR experiments. The reaction rates for the formation of a crosslink and the formation of a cyclic sulfide will be similar, since the chemistry is similar. Thus, the relative formation of cyclic sulfides versus crosslinks will be proportional to the probability by which the radical attaches to the neighboring chain versus looping back to the backbone chain. We define the looping probability as the probability of attaching to an allylic carbon on the original rubber backbone that is within a sphere with a radius equal to that of the length of the sulfur chain. The complement of the looping probability is the crosslinking probability, which refers to the probability with which the persulfenyl radical will attach to the allylic carbon on a neighboring isoprene chain. In natural rubber it can be assumed that the concentration of allylic sites is sufficient that the persulfenyl radical can always react; thus, the sum of the looping and crosslinking probabilities is unity.

The basic idea needed to calculate the looping probability is the probability that a sulfur radical with $y$ atoms that is connected to an $n$-mer isoprene chain, can interact with a segment of the isoprene chain. If an allylic site on the backbone chain is found, a loop can be formed. This idea is shown schematically in Figure 9. In order to determine the looping probability, the probability of various conformations of both the polysulfidic and the polymer chain will be required. The probability $\mathrm{P}\left(\mathbf{s}_{\mathrm{k}}\right)$ that the $k^{\text {th }}$ chain segment is at a distance $\mathbf{s}_{\mathrm{k}}$ from the center-of-mass of the chain was derived by Isihara ${ }^{123}$ and by Debye and Bueche ${ }^{124}$ and is given by:

$$
\mathrm{P}\left(\mathbf{s}_{\mathrm{k}}\right)=\left\{\frac{2 \pi \mathrm{R}^{2}}{9}\left[\mathrm{u}^{3}+(1-\mathrm{u})^{3}\right]\right\}^{-3 / 2} \exp \left(\frac{-9 \mathbf{s}_{\mathrm{k}}{ }^{2}}{2 \mathrm{R}^{2}\left[\mathrm{u}^{3}+(1-\mathrm{u})^{3}\right]}\right)
$$

where $\mathrm{R}^{2}$ is the chain's mean square end-to-end distance, $u=k / n$, and $n$ is the number of segments in the chain. Since we need to know the probability that any segment is at a distance $s$ from the center of mass of the chain, Equation 43 needs to be integrated over all the segments, i.e. for all $u \in[0 \leq u \leq 1]$. In calculating the looping probability, we are concerned only with calculating the number of allylic carbons at a given distance $s$ from the center of mass of the chain. Since for isoprene monomers exactly half the number of backbone carbons are allylic, the looping probability will be one half the probability of finding any segment at a given distance $s$ from the center of mass of the chain. The carbon in the methyl group does not contribute to the contour of the rubber chain; however, it does affect the local conformations and thus the size of the isoprene chain in the rubber. The analytical expression given in Equation 43 is an asymptotic 
expression that is valid only for long Gaussian chains (i.e. chains with 30 or more repeat units). Thus, for polysulfidics containing 1 to 16 sulfur atoms, application of Equation 43 may not be appropriate, and a more detailed conformational analysis of persulfenyl radicals that is valid for all the lengths will be required. In the remainder of this Section, we will first consider the independent conformations of the isolated sulfur and isoprene chains; then, we will analyze the joint probability of the sulfur and isoprene chains in order to determine the looping probability.

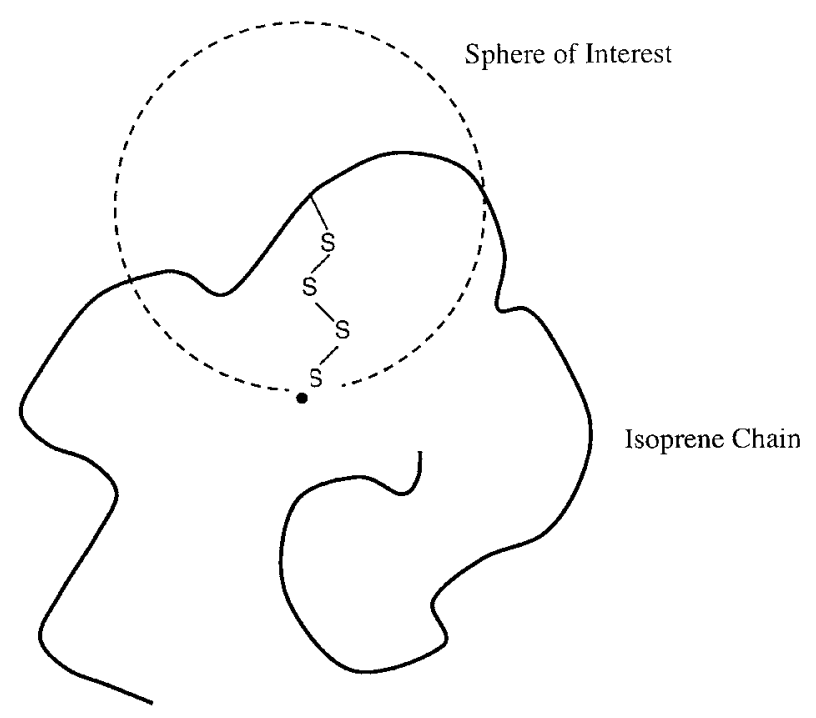

FIG. 9. - Schematic of the sphere of interaction of the persulfenyl radical R-S ${ }_{x}$ with the allylic carbons in the isoprene chain.

\section{A. CONFORMATIONAL ANALYSIS OF SULFUR AND ISOPRENE CHAINS}

The spatial locations of the various atoms in a polymer chain are characterized by the sequence of three parameters - the bond length, the bond angle $(\theta)$, and the dihedral angle or the torsional angle $(\phi)$. The rotation around a single bond leads to a distribution of dihedral angles, some of which correspond to the minimum energy conformations, which are highly probable. Based upon the steric effects and excluded volume interactions with the neighboring atoms, every conformation is characterized by an energy, which determines the probability of the existence of the molecule in that particular conformation. In order to enumerate all the different possible conformations, the rotational isomeric state approximation ${ }^{125}$ will be employed.

Several reviews on elemental sulfur and its properties are available. ${ }^{126-128}$ The bond length of an S-S bond is $2.06 \mathrm{~A}^{\circ}( \pm 0.02)$ and the bond angle $\theta=74^{\circ}( \pm 2) .{ }^{129} \mathrm{Ab}$-initio calculations of torsional angles for polysulfanes $\left(\mathrm{H}_{2} \mathrm{~S}_{\mathrm{n}}, \mathrm{n}=2 \ldots 6\right)$ has been reported by Suleimenov and Tae-Kyu Ha. ${ }^{130}$ The rotational barrier about an S-S bond in a sulfur chain is found to be between 6 and 10 $\mathrm{kcal} / \mathrm{mol}^{130-132}$ as compared to that of the rotational barrier of the central C-C bond of butane, which is around $4 \mathrm{kcal} / \mathrm{mol}$. This means that the $\mathrm{S}-\mathrm{S}$ bond lengths and bond angles are effectively rigid and only bond rotations need to be considered. The minima in the rotational potentials are located at $\phi= \pm 90^{\circ}$ where $\phi=0$ corresponds to the trans state where all the $\mathrm{S}$ bonds are in the same plane. The rotational energy barrier is considerably greater than $\mathrm{kT}$ at normal temperatures and hence the rotation of the sulfide linkages can be assumed to be in discrete rotational isomeric states. ${ }^{125}$ The conformation that is a $90^{\circ}$ clockwise rotation from the reference trans state will be designated as + , while the $90^{\circ}$ counter-clockwise rotation will be - .

A sulfur chain with $n$ atoms has (n-1) bonds and (n-3) dihedral angles. Any conformation 
can be represented by a sequence of (n-3) clockwise (+) or counter clockwise (-) rotations of the dihedral angle; thus, there are $2^{(\mathrm{n}-3)}$ conformations in a sulfur chain of length $n$. The energy of each of these conformations is calculated by summing the conformational energies of all the constituent bonds, assuming that the energies are independent and that the effect of a bond is not felt at more than a distance of three neighboring bonds ${ }^{125}$. The probability of a particular conformation, $\mathbf{x} \in( \pm, \pm, \ldots)$ is given by

$$
p(x)=\frac{e^{\frac{-E_{x}}{R T}}}{\sum_{x} e^{\frac{-E_{x}}{R T}}}
$$

where $E_{x}$ is the energy of the conformation, $\mathbf{x}$. All possible conformations of sulfur chains up to a length of 17 atoms have been enumerated and the probability distribution as a function of the end-to-end distances has been determined. A typical example of such a calculation for the probability distribution for $\mathrm{S}_{17}$ at $150{ }^{\circ} \mathrm{C}$ is shown in Figure 10, where the most probable conformation has an end-to-end distance between 8 and $9 \AA$. The probability distribution in Figure 10 is not a simple peak, but rather shows a fine structure. This is not a consequence of sampling a limited population of conformers since all the conformers of $S_{17}$ were enumerated; rather, because of the restricted number of bond and torsional angles, certain conformations require a higher energy sequence of bonds and consequently certain end-to-end distances are not as probable.

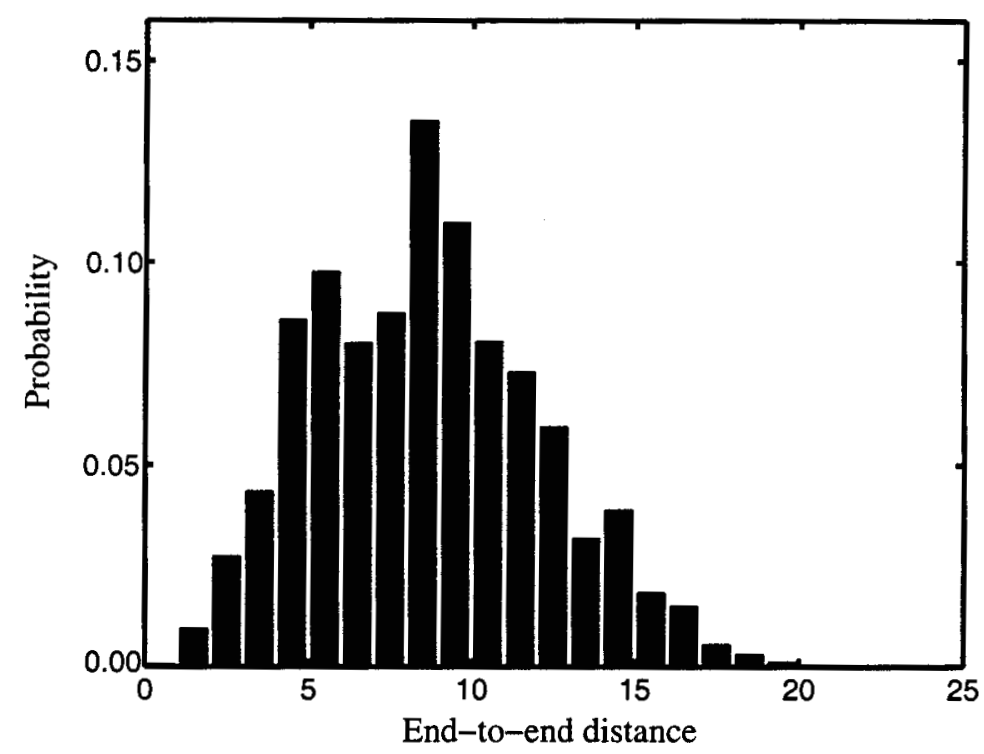

FIG. 10. - The probability distribution of end-to-end distances for all the conformations of $\mathrm{S}_{17}$ chain.

The conformational statistics were also determined for natural rubber, or equivalently cis1,4-polyisoprene, which has a molecular structure of $-\left[\mathrm{CH}_{2}-\mathrm{C}\left(\mathrm{CH}_{3}\right)=\mathrm{CH}-\mathrm{CH}_{2}-\right]_{\mathrm{x}}$. The local conformation of the isoprene repeat unit is shown in Figure $11,{ }^{133}$ which leads to a simplified representation of a sequence of any mer as a combination of three bonds, $\mathrm{U}_{\mathrm{a}}, \mathrm{U}_{\mathrm{b}}$ and $\mathrm{U}_{\mathrm{c}}$. Three different configurations i.e. $-60^{\circ}, 0^{\circ}$ and $60^{\circ}$ are energetically favorable for the bond $U_{a}$. The adjacent bond $\mathrm{U}_{\mathrm{b}}$ can also exist in the above three conformations, except $\mathrm{U}_{\mathrm{b}}$ and $\mathrm{U}_{\mathrm{a}}$ cannot both 
lie in the $0^{\circ}$ local conformation due to steric effects. Bond $\mathrm{U}_{\mathrm{c}}$ can be in $-120^{\circ}, 0^{\circ}$ and $120^{\circ}$ conformations irrespective of the conformations of the other bonds. Therefore, the sequence of three bonds, which correspond to one mer in the polyisoprene chain, can exist in a total of 24 different conformations. The simplest case of 3 mers, which can be represented as $\mathrm{UbUc}(\mathrm{UaUbUc}) \mathrm{UaUb}$, can exist in $1728(8 \times 24 \times 9)$ different conformations. Generalizing this, we conclude that there are a total of $8 \times 24^{(\mathrm{n}-2)} \times 9$ conformations for an $n$-mer, which for a 7-mer would be approximately 570 million. Using the conformational analysis for a 7-mer chain of isoprene units, the number of allylic carbons inside a radius $r$ were determined for all the possible 570 million conformations.
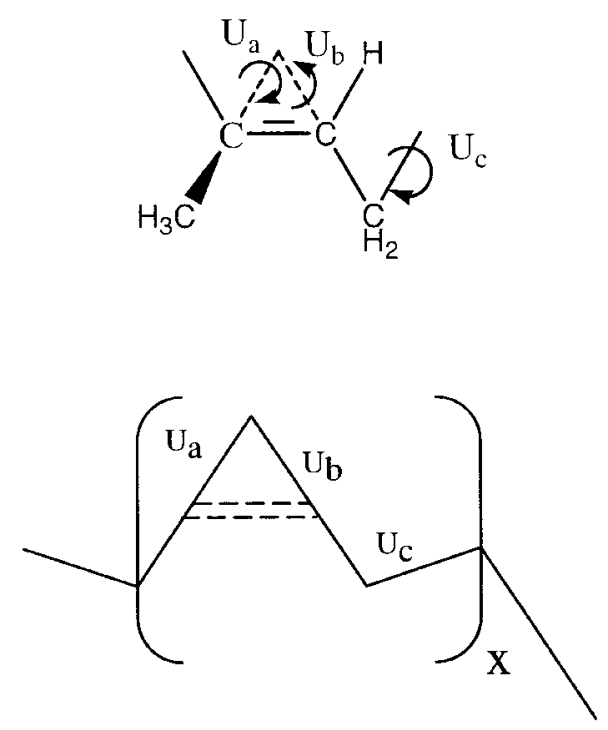

FIG. 11. - Geometric representation of an isoprene chain. The dotted lines from between $U_{a}$ and $U_{b}$ represent the double bond and the allowed angles of $\mathrm{U}_{\mathrm{a}}$ and $\mathrm{U}_{\mathrm{b}}$ incorporate the rigid nature of the trans double bond. ${ }^{133}$

\section{B. ESTIMATION OF LOOPING PROBABILITIES}

We are now in the position to estimate the looping probability. The looping probability will be determined using the following procedure:

1. The origin is defined as the point of attachment of the polysulfidic radical to the isoprene chain, which is also assumed the center of the isoprene chain.

2. Using conformational analysis of the isoprene chains, the spatial locations of each of the isoprene segments on an isoprene chain are determined, and the number of segments of the isoprene chain of $n$-mers that exist at any given distance from the origin in a particular conformation can be calculated. This number is defined as $\mathrm{C}_{\mathrm{nr}}$, where the subscript $n$ represents the number of mers considered in the isoprene chain and subscript $r$ represents the radial distance from the origin.

3. $\mathrm{C}_{\mathrm{nr}}$ is now multiplied by the probability of occurrence of the corresponding conformation given by Equation 44 and is summed over all the possible $8 \times 24^{(n-2)} \times 9$ conformations. This yields the average number of segments $\left(\mathrm{N}_{\mathrm{avg}}\right)$ at any given distance $r$ on an isoprene chain of $n$-mers. Thus

$$
\mathrm{N}_{\mathrm{avg}}=\sum_{\mathrm{X}} \mathrm{C}_{\mathrm{nr}} \mathrm{p}(\mathrm{x})
$$


4. Conformational analysis on the sulfur chains with $y$ atoms is used to calculate $\psi(r, y)$ which is the probability of a sulfur chain of $y$ atoms to have an end-to-end distance between $r$ and $r+d r$, where $d r=1 \mathrm{~A}^{\mathrm{o}}$.

5. We have assumed that the sulfur chain is attached to the center of the isoprene chain. Consequently, the looping probability of the sulfur chain with $y$ atoms with a given end-to-end distance $r$ is one half the probability of finding an isoprene segment at the distance $r$ and is equal to $(1 / 2) N_{a v g} x \psi(r, y)$. Since we need to consider all $r$ varying from 0 to $\infty$, the net looping probability is given by

$$
\sum_{\mathrm{r}=0}^{\infty} \frac{1}{2} \mathrm{~N}_{\mathrm{avg}} \psi(\mathrm{r}, \mathrm{y})
$$

In Figure 12, the looping probabilities are plotted for sulfur chains $S_{x}$ from $x=1, \ldots, 17$ for three different isoprene chains of 5, 6 and 7 mers. For the purpose of comparison, the results are also shown using the infinite chain analytical expression given by Equation 43 for an isoprene with 7 and 20 mers. From Figure 12 it is clear that the looping probability of the sulfur radicals of smaller lengths up to $\mathrm{S}_{4}$ can be as high as $15 \%$, but for longer chains the looping probability is between 3 to $5 \%$. This is reasonable since the probability of a sulfur radical finding an unsaturation on the backbone is greater when the chain is smaller. However, the looping probability of a sulfur chain with one sulfur atom is less than that of the other shorter chains like $S_{2}, S_{3}$ and $\mathrm{S}_{4}$ due to geometric constraints that makes it difficult for the chain with a single sulfur atom to loop back onto the isoprene backbone to which it is attached. Looping in this case would be possible only if the conformation of the isoprene chain is such that it locates itself favorably close to the sulfur chain.

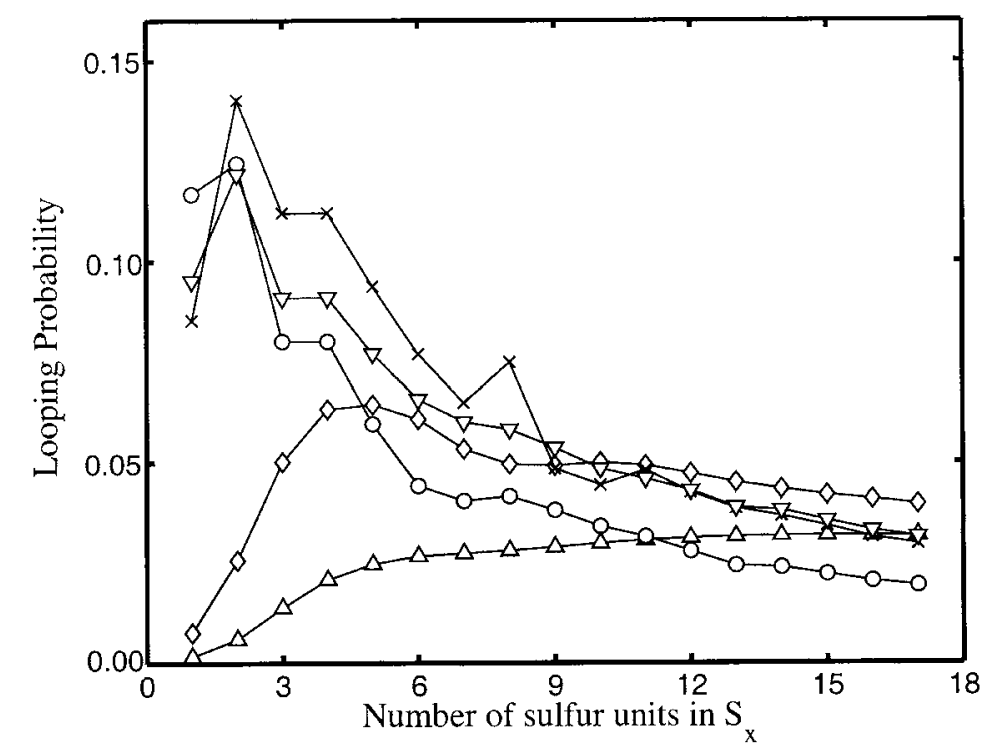

FIG. 12. - Looping probability for sulfur chains of various lengths. Numerical simulations for isoprene: 5-mer (o), 6-mer (x) and 7-mer $(\nabla)$ and analytical results using Equation 43 for a 7-mer $(\diamond)$ and 20-mer $(\Delta)$. Lines have been drawn for visual clarity.

Comparing the numerical results for an isoprene chain of 7-mers with that of the asymptotic relation for long Gaussian chains determined using Equation 43, it is evident that the numeri- 
cal results are comparable to that of the analytical expression for sulfur chains with 10 to 17 sulfur atoms. This indicates that the detailed numerical simulation is accurate. Ideally, the looping probability should be estimated for an isoprene chain of infinite length; however, for a 7-mer isoprene chain it takes 720 hours on a $600 \mathrm{MHz}$. Pentium III machine, making calculations for longer chains impractical. Consequently, an infinite chain is being approximated with a 7-mer isoprene chain. As shown in Figure 12, there are only marginal differences between the results using a 6-mer compared to a 7-mer and at large $x$ 's the predicted looping probabilities using the detailed 7-mer analysis converge with the analytical treatment for 7 and 20 -mer. Thus, we believe that the detailed conformational analysis for 7-mers in Figure 12 is sufficient to compute the looping probabilities for what really is an infinite length isoprene chain.

The analysis described in this Section provides a rational framework to determine the looping probability of different polysulfidic radicals attached to an isoprene backbone. The results clearly indicate that the probability of looping decreases as the number of sulfur atoms in the polysulfidic radical increases, where shorter polysulfidic radicals have looping probabilities as high as $15 \%$, while longer radicals typically have lower probabilities of 5\% or less. Considering the approximations made in this analysis, it is reasonable to conclude that on the average the looping probability of persulfenyl radicals with number of sulfur atoms in the range of 1 to 17 is approximately $10 \%$. Of course, the population balance equations can be readily generalized to accommodate variations in the looping probability with the length of the persulfenyl radical if needed.

\section{EXPERIMENTAL}

Experimental data is needed to determine the rate constants and to validate the predictions of the population balance model. ODR measurements have been employed to determine the time evolution of the concentration of crosslinks for different formulations cured at different temperatures. From the statistical theory of rubber elasticity, ${ }^{134}$ the shear modulus $\mathrm{G}$ is proportional to the number of network chains per unit volume and the absolute temperature T. If a network is formed from a polymer of infinite molecular weight so that the chain ends can be ignored and if the crosslinks are tetrafunctional, then

$$
\mathrm{G}=2 \vartheta \mathrm{kT}
$$

where $v$ is the number of crosslinks present per unit volume and $\mathrm{k}$ is the Boltzmann constant. In the ODR experiment, G evolves with time as the cure advances; consequently,

$$
v(\mathrm{t})=\frac{\mathrm{G}(\mathrm{t})}{2 \mathrm{kT}}
$$

Since the elastomer prior to vulcanization is entangled, the contribution of the entanglements to the shear modulus measurements must be accounted for in order to determine the evolution of the chemical crosslinks. Thus we assume that

$$
v(\mathrm{t})=\frac{\Delta \mathrm{G}(\mathrm{t})}{2 \mathrm{kT}}
$$

where and $\mathrm{G}_{\min }$ is the minimum modulus in the ODR curve at short times as shown in Figure 3.

A number of natural rubber formulations were investigated. All formulations contained $5 \mathrm{phr}$ of $\mathrm{ZnO}, 2.5 \mathrm{phr}$ of stearic acid and $3 \mathrm{phr}$ of 6PPD (i.e. N-(1,3-dimethylbutyl)-N'-phenyl-p- 
phenylenediamine) antioxidant. Formulations were prepared with $0.5,0.75$ and $1 \mathrm{phr}$ of the MBS accelerator, 2 and 4 phr of sulfur and with $0.0,0.1,0.2$ and $0.3 \mathrm{phr}$ of CTP retarder. Carbon black was not used in any of the formulations. Samples were made by first mixing a master batch of all ingredients except the sulfur, accelerator and retarder in a BR Banbury mixer for 5 to 6 minutes until the batch temperature reached $300^{\circ} \mathrm{F}$. The batch was then masticated on a two-roll mill for eight passes, and then allowed to cool to room temperature. The sulfur, accelerator and retarder were added as the sample was again masticated on a two-roll mill with a rolling bank, where there were two cut downs and eight additional passes. The material was then stored at room temperature. The ODR curve was then determined at $298^{\circ} \mathrm{F}, 310^{\circ} \mathrm{F}$ and $330^{\circ} \mathrm{F}$ using a rheometer that was operated at $100 \mathrm{cpm}$ with a 0.5 degree displacement.

\section{RESULTS}

We are now prepared to critically examine the capabilities of the PBEs to describe the ODR data for MBS accelerated sulfur vulcanization of natural rubber. Our objective in this Section is to (i) describe the observed time dependant formation of the total crosslink density (i.e. the sum of all the $\mathrm{Vu}_{\mathrm{x}}$ species) for various formulations and (ii) examine the temporal evolution of the other polysulfidic species predicted by the PBEs. This Section will be organized as follows. In Section VII.A the predictions will be examined for Model-I where sulfur is picked-up as $\mathrm{S}_{8}$, and in Section VII.B the predictions for Model-II where sulfur is picked-up sequentially will be presented. In Section VII.C we will present a rigorous statistical analysis of the error bounds on the kinetic parameters and will show that the rate constants have been robustly determined for the various reaction classes.

\section{A. PREDICTIONS FOR MODEL I: SULFUR PICK-UP AS $\mathrm{S}_{8}$}

The Model-I PBEs for the vulcanization reactions, which assume the accelerator picks up sulfur as $\mathrm{S}_{8}$ (i.e. R.19), are defined by Equations 5 through 29. Model-I has a total of 100 nonlinear-coupled ordinary differential equations for systems without the retarder. When the retarder is present, Equations 27 through 29 must also be included. Although there are 100 differential equations (or 102, when retarder is present) which model the evolution of the different species that participate in the vulcanization reactions, the total number of reactions is of the order of several hundreds. Due to the explosion in the number of reactions with increasing polysulfidic lengths, the maximum chain length of the polysulfides has been restricted to 16; however, the framework can be readily extended to include longer polysulfidic chains if needed.

In order to solve the PBEs, the rate constants for the various reactions must be determined from the ODR data. Making the simplifying assumption that the rate constants are independent of polysulfidic length, there are 15 different types of reactions and, thus, 15 different rate constants must be determined. The determination of 15 rate constants by non-linear optimization is difficult; therefore, the number will be reduced by imposing relationships between some rate constants that are consistent with the underlying reaction mechanisms. The rate constant $\mathrm{k}_{\mathrm{E}-\mathrm{R}}$ is set equal to $\mathrm{k}_{\mathrm{VU}}$, since both these rate constants govern the reaction of an active polysulfidic radical with an allylic carbon on an isoprene chain. When the polysulfidic radical is of sufficient length (i.e. more than 4 active sulfur atoms), the reactivity for $\mathrm{RS}_{\mathrm{x}}{ }^{\bullet}$ and $\mathrm{BtS}_{\mathrm{x}}{ }^{\cdot}$ should be identical, since the end-groups should have a minimal affect on the radical's reactivity, although for shorter chain lengths this assumption may not be appropriate. If information on how the end group affects the reactive radical is available from either experimental data or quantum chemistry calculations, the effect of the end-group on the rate constant can be readily incorporated. But for the present analysis, the effect of end groups on radical reactivity is not considered; thus, $\mathrm{k}_{\mathrm{E}-\mathrm{R}}$ is set equal to $\mathrm{k}_{\mathrm{VU}}$. Likewise, the rate constants $\mathrm{k}_{\mathrm{BST}-\mathrm{S}}$ and $\mathrm{k}_{\mathrm{E}-\mathrm{S}}$ are set equal, since these two rate 
constants are for sulfur-activation by a polysulfidic radical. Both $\mathrm{k}_{\mathrm{A}-\mathrm{BST}}$ and $\mathrm{k}_{\mathrm{DESULF}}$ are set equal to $\mathrm{k}_{\mathrm{A}-\mathrm{A}}$, since these three rate constants essentially control the rate of exchange of sulfur between polysulfides. Finally, $\mathrm{k}_{\mathrm{LOOP}}=0.1 \times \mathrm{k}_{\mathrm{VU}}$ based on the discussion in Section 5. Using these simplifications, a total of nine rate constants must be determined: $\mathrm{k}_{\mathrm{MBS}}, \mathrm{k}_{\mathrm{MBS}-\mathrm{MBT}}, \mathrm{k}_{\mathrm{A}-\mathrm{S}}, \mathrm{k}_{\mathrm{A}-\mathrm{A}}, \mathrm{k}_{\mathrm{A}-\mathrm{R}}$, $\mathrm{k}_{\mathrm{B}-\mathrm{R}}, \mathrm{k}_{\mathrm{VU}}, \mathrm{k}_{\mathrm{DEG}}$ and $\mathrm{k}_{\mathrm{E}-\mathrm{S}}$. The rate constants and the associated activation energies are determined via optimization of the ODR data for five different formulations at three different temperatures. The optimization involves minimizing the sum-of-the-squares of the difference between the experimental observations and the total concentration of crosslinks predicted by the model for a discrete series of time points and the different compositions and temperatures. The objective function is given by

$$
\mathrm{F}\left(\mathrm{k}_{\mathrm{f} 1}, \mathrm{k}_{\mathrm{f} 2}, \ldots \ldots, \mathrm{k}_{\text {sulf }}\right)=\sum_{\text {formulations }} \sum_{\mathrm{i}=1}^{\mathrm{q}}\left[\mathrm{Vu}_{\mathrm{i}, \mathrm{e}}^{\mathrm{total}}-\mathrm{Vu}_{\mathrm{i}, \mathrm{p}}^{\mathrm{total}}\right]^{2}
$$

where $\mathrm{Vu}_{\mathrm{i}, \mathrm{e}}{ }_{\text {total }}$ and $\mathrm{Vu}_{\mathrm{i}, \mathrm{p}}$ total are the experimental and model predictions respectively and there are $\mathrm{q}$ time points in each individual ODR curve. The Levenberg-Marquadt algorithm ${ }^{135}$ as implemented in MATLAB ${ }^{\circledR}$ is employed for optimization. The optimization is performed at three different temperatures to obtain the rate constants at each of the temperatures, which are subsequently parameterized into pre-exponential factors and the associated activation energies. The pre-exponential factors and the corresponding activation energies for the nine rate constants are tabulated in Table VII for Model-I.

TABLE VII

ACtivation Energy and Pre-Exponential Factors For Model-I

\begin{tabular}{ccc}
\hline Rate-Constant & Pre-Exponential Factor & Activation Energy (kcal/mol) \\
\hline $\mathrm{k}_{\mathrm{MBS}}$ & $6.726 \times 10^{7}$ & 19.7 \\
$\mathrm{k}_{\mathrm{MBS}-\mathrm{MBT}}$ & $1.197 \times 10^{4}$ & 8.8 \\
$\mathrm{k}_{\mathrm{A}-\mathrm{S}}$ & $1.191 \times 10^{4}$ & 6.7 \\
$\mathrm{k}_{\mathrm{A}-\mathrm{A}}$ & $2.394 \times 10^{17}$ & 32.9 \\
$\mathrm{k}_{\mathrm{A}-\mathrm{R}}$ & $3.646 \times 10^{13}$ & 26.0 \\
$\mathrm{k}_{\mathrm{B}-\mathrm{R}}$ & $4.236 \times 10^{25}$ & 49.2 \\
$\mathrm{k}_{\mathrm{VU}}$ & $1.120 \times 10^{6}$ & 8.9 \\
$\mathrm{k}_{\mathrm{DEG}}$ & $3.568 \times 10^{16}$ & 35.7 \\
$\mathrm{k}_{\mathrm{E}-\mathrm{S}}$ & $2.850 \times 10^{5}$ & 10.9 \\
\hline
\end{tabular}

In Figure 13, the concentration profile predicted by the population balance model for the total concentration of crosslinks is compared with that of the isothermal ODR data at $330^{\circ} \mathrm{F}$ for five different formulations with varying amounts of accelerator and sulfur. The model is able to adequately describe the vulcanization process, including the initial scorch delay, cure and the post-cure (i.e. reversion). The Model-I PBEs are able to describe the important features of the net production of crosslinks in the vulcanization process for a wide range of accelerator and sulfur compositions; however, certain features of the crosslink development are not perfect. The scorch delay predicted by Model-I shows that initially there is no production of crosslinks; however, the experimental data initially exhibit a slow, but steady, increase in the crosslink density. This dis- 
crepancy is not an artifact of the parameter optimization, since no set of rate constants could be found that was able to resolve this particular feature of the cure-curve without significantly compromising other aspects of the production. The fact that Model-I predicts that crosslinks are not formed at short times is not surprising, considering that crosslinks can only form after the accelerator and sulfur have reacted to such an extent that crosslink precursors like B and $\mathrm{B}^{*}$ are formed in sufficient concentrations. There are two possible explanations for the origin of the discrepancy in the initial foot of the Vu predictions as compared to the data. First, the number crosslinks is inferred from the shear modulus determined via ODR, where the kinetic analysis assumes isothermal conditions; however, the loading and the thermal equilibration of the sample in the ODR require a finite time and, thus, the definition of $t=0$ is somewhat arbitrary. We have defined $\mathrm{t}=0$ as the time when the shear modulus is at its minimum (see Figure 3 ) which is between 10 to 20 secs after the sample is placed in the ODR. Since the sample is being heated during this initial time period, the vulcanization reactions will have already started prior to $\mathrm{t}=0$; consequently, the experimental scorch delay that is reported in Figure 13 may be shorter than it would occur for a truly instantaneous change in temperature at $t=0$. Alternatively, the initial gradual increase in the concentration of crosslinks followed by a sharper rise as indicated in the experimental ODR response may be indicative of two different kinds of polysulfidic accelerators. As discussed previously in Section III.A.4, during the early stages of the cure, zinc-free accelerators (i.e. BtS-S $\mathrm{X}_{\mathrm{X}} \mathrm{SBt}$ ) most probably dominate, while during the later stages when MBT is available, zinc-complexed species (i.e. BtS-S ${ }_{X}-\mathrm{Zn}-\mathrm{SBt}$ ) are also formed. Since zinc-free accelerators have slower reaction rates as compared to zinc-complexed accelerators, there should be an initial slow rise in the total $\mathrm{Vu}$ production followed by an increase in the rate of $\mathrm{Vu}$ production. However, the current set of PBEs would not capture this difference, since the reactivity of zinc-free and the zinc-complexed species is assumed to be the same by Assumption 4 in Section IV. One could of course differentiate between zinc-free and zinc-complexed structures in the PBEs, but this would require the introduction of additional rate constants, which we do not believe is warranted at this time. Model-I also predicts the reversion process, although the model predicts a softer transition from the curing process to the reversion process than is observed experimentally. The only mechanism for reversion that is included in Model-I is the formation of elastically ineffective loops as discussed in Section V. In addition, the looping probability is anticipated to be a function of the length of the $\mathrm{Vu}_{\mathrm{x}}$ chains as shown in Figure 6; however, in the model we have assumed the looping probability to be constant at $10 \%$ and irrespective of the chain length. Moreover, the predicted reversion depends upon the details of the distribution of $\mathrm{Vu}_{\mathrm{x}}$; consequently, any shortcomings of Model-I in the reactions that precede the formation of the crosslinks can affect the reversion process. Notwithstanding the afore mentioned difficulties, Model-I is able to capture all the important features of the ODR data for a range of accelerator and sulfur concentrations, using a reaction mechanism that is chemically reasonable. 


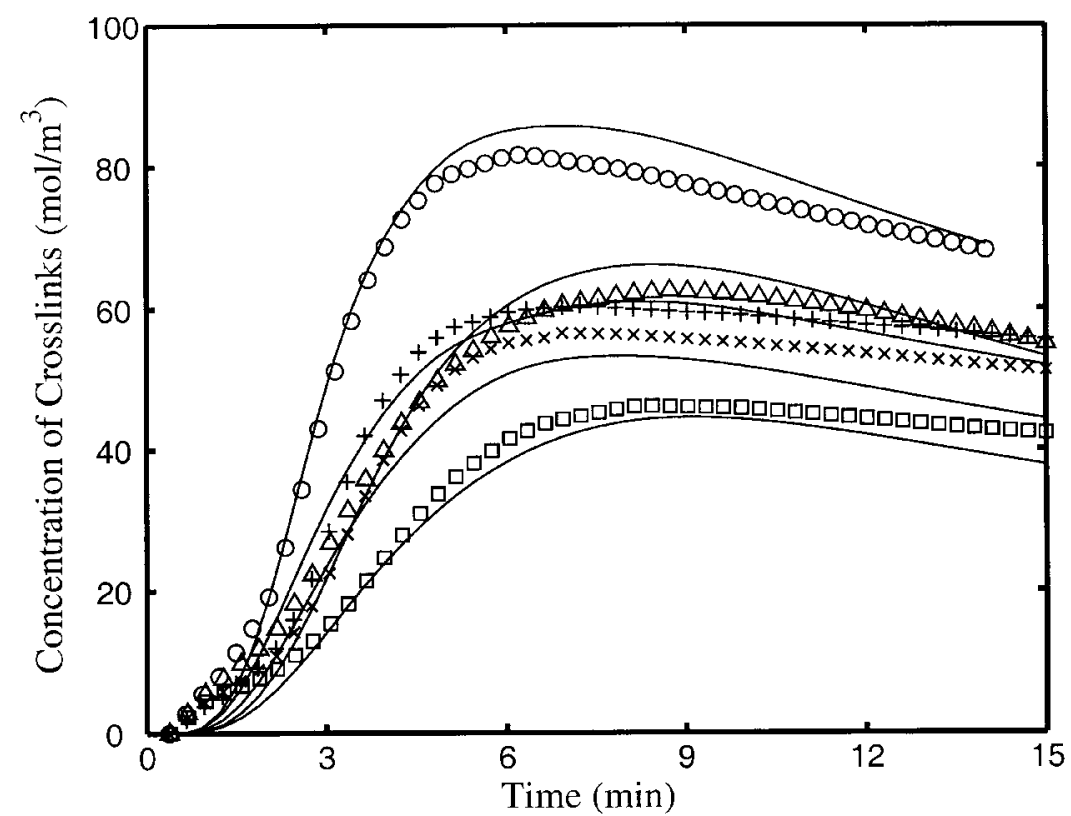

FIG. 13. - Comparison of the temporal evolution of the total (i.e. sum of all $\mathrm{Vu}_{\mathrm{x}}$ 's) concentration of crosslinks predicted by Model-I with experimental ODR data for different formulations cured at $330^{\circ} \mathrm{F}$.

Formulations include: (○) $1 \mathrm{phr}$ MBS and $4 \mathrm{phr} \mathrm{S}$; (+) $1 \mathrm{phr}$ MBS and $2 \mathrm{phr} \mathrm{S} ;(\triangle) 0.5 \mathrm{phr}$ MBS and $4 \mathrm{phr} \mathrm{S}$; (x) $0.75 \mathrm{phr}$ MBS and $2 \mathrm{phr} \mathrm{S}$; $\square$ ) $0.5 \mathrm{phr}$ MBS and $2 \mathrm{phr}$ S. The predictions are indicated by the solid lines. No retarder is used and the rest of the cure-system is given in Section 6.

Since the ODR measures the total concentration of crosslinks of different lengths, the predictions from the Model-I shown in Figure 13 are the lumped concentration of the crosslinks, i.e. the chain length dependence of the crosslinks is not considered. Similar lumped concentrations for other important species including A, B and $\mathrm{B}^{*}$ are shown in Figure 14, as well as the consumption of the accelerator MBS and sulfur $\mathrm{S}_{8}$. It should be noted that $\mathrm{A}, \mathrm{B}$ and $\mathrm{B}^{*}$ are defined as the total number of moles of the various species, not by the total mass of sulfur that is present in A, B and $\mathrm{B}^{*}$. As expected both the concentration of sulfur and MBS decrease monotonically during the course of the reaction. Although the activated species A are initially zero, they are formed immediately upon initiation of the reaction. The concentration of A reaches a maximum at approximately 5 minutes after initiation of the cure process. Both $\mathrm{B}$ and $\mathrm{B}^{*}$ show peaks in the concentration between 2 and 3 minutes, where the B peak occurs a little later than the $\mathrm{B}^{*}$ peak. During the early stages of the cure, the concentration of $\mathrm{B}^{*}$ is greater than that of $\mathrm{B}$, which may appear to be somewhat surprising since $\mathrm{B}^{*}$ is formed from $\mathrm{B}$. However, this is a consequence of the fact that $\mathrm{B}^{*}$ is also formed by sulfur activation via $\mathrm{R} .50$ in addition to the formation from $\mathrm{B}$. Thus, although $\mathrm{B}^{*}$ is formed from $\mathrm{B}$ at the beginning, it quickly exceeds the concentration of $\mathrm{B}$ since it grows simultaneously from sulfur activation during the early stages of the cure, when sulfur is present in significant concentrations. At longer times when most of the sulfur is depleted, the concentration of $\mathrm{B}^{*}$ is less than that of $\mathrm{B}$ as expected. 


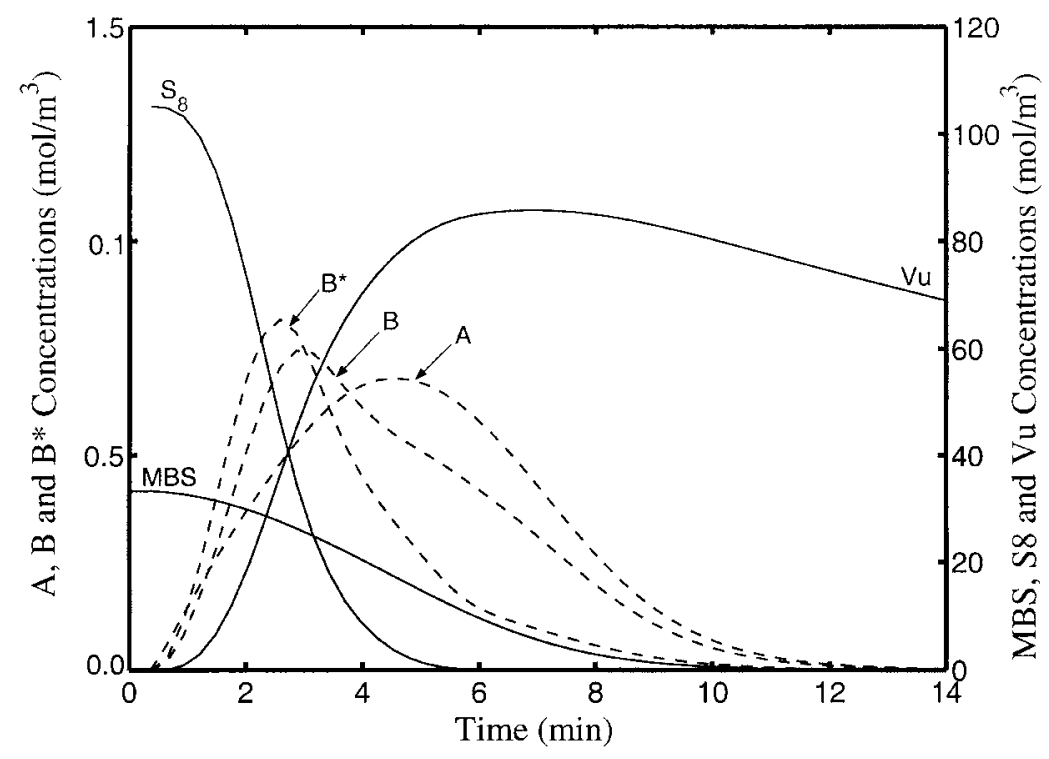

\footnotetext{
FIG. 14. - Prediction of Model-I for the temporal evolution of lumped species $\mathrm{A}=\sum_{x=0}^{14} A_{x}, \mathrm{~B}=\sum_{x=1}^{16} B_{x}$, $\mathrm{B}^{*}=\sum_{x=1}^{16} B_{x}^{*}$ and $\mathrm{Vu}=\sum_{x=0}^{16} V u_{x}$. Predictions are for a system with $1 \mathrm{phr}$ of MBS accelerator and $4 \mathrm{phr}$ of sulfur that was cured isothermally at $330^{\circ} \mathrm{F}$.
}

The Vu profile shows an initial delay for approximately 0.5 minutes and then a rapid rise to a maximum at approximately 6 minutes. The initial foot in the $\mathrm{Vu}$ response (i.e. the scorch delay) may appear surprising, since the reactive crosslink precursor $\mathrm{B}^{*}$ is formed very rapidly upon initiation of the reaction; however, this result is consistent with R.53. Specifically, once B ${ }^{*}$ has been formed it reacts with MBTS (i.e. $A_{0}$ ) and only after depletion of most of the MBTS can $B^{*}{ }_{x}$ produce crosslinks. Finally, the concentration of the lumped Vu species decreases slowly after its maximum at 6 minutes due to the formation of dead ends and elastically ineffective loops. The formation of these dead ends and elastically ineffective loops occurs throughout the cure process; however, the rate of crosslink formation is initially much greater than the rate of loop formation so that the concentration of $\mathrm{Vu}$ increases for times less than 6 minutes. The PBE model results shown in Figure 13 qualitatively agree with the lumped parameter simulations of Coran $^{21}$ and Ding et $a l .{ }^{24,25}$ However, in contrast to the lumped parameter models of Coran and coworkers, which require the initial concentration of $\mathrm{A}$ as input, the population-balance model predicts the formation of A from the initial concentrations of accelerator and sulfur. In addition the population-balance model is consistent with the experimental observation that sulfur is totally consumed at the end of the reaction.

Since the population balance equations explicitly account for polysulfides of different lengths, the evolution of the individual species $\mathrm{A}_{\mathrm{x}}, \mathrm{B}_{\mathrm{x}}, \mathrm{B}_{\mathrm{x}}{ }_{\mathrm{x}}$ and $\mathrm{Vu}_{\mathrm{x}}$ are also predicted. In Figure 15 the time evolution of the crosslinks of different lengths is given. The crosslink distribution for cure times of less than $2 \mathrm{~min}$ is initially biased towards the longer length crosslinks (i.e. $\mathrm{Vu}_{\mathrm{x}}$ with $\mathrm{x}$ greater than 8 ); however, with increasing time the longer length crosslinks desulfurate to shorter lengths, shifting the distribution towards shorter polysulfidic crosslinks. The formation of longer crosslinks initiates around $0.6 \mathrm{~min}$ and reaches a maximum at around $4 \mathrm{~min}$ followed by the shorter length crosslinks which initiate around $1 \mathrm{~min}$ and peak around $5.5 \mathrm{~min}$. Since monosulfidic crosslinks do not desulfurate and are more stable than the polysulfidic crosslinks, their concentration is eventually greater than any other polysulfidic length. 


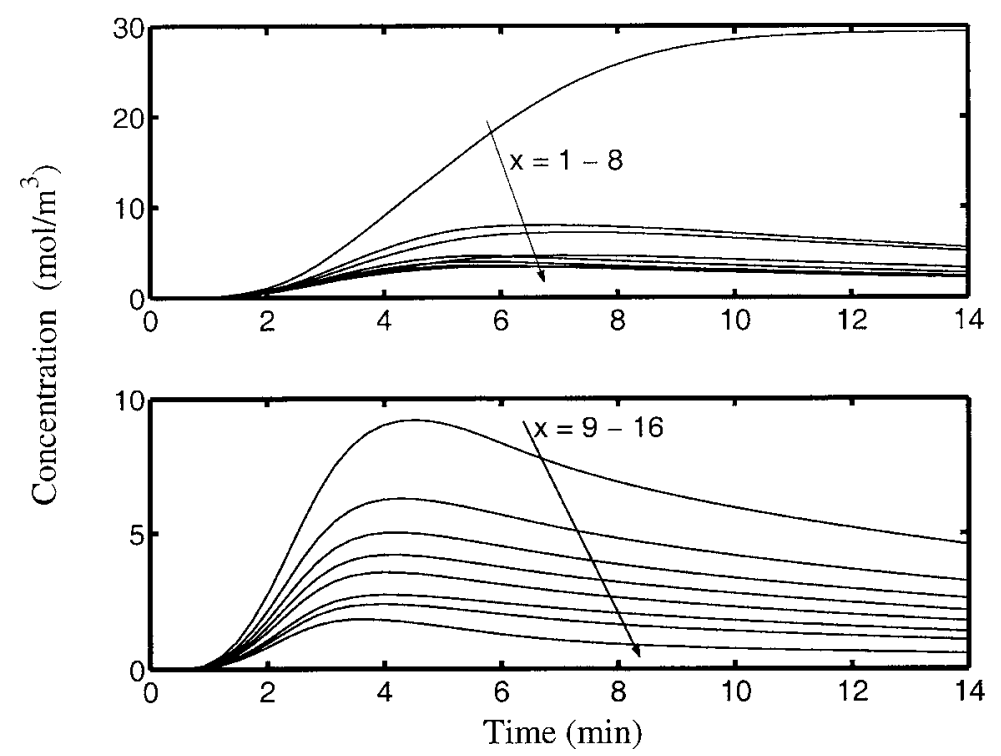

FIG. 15. - Temporal evolution of crosslinks of different lengths predicted by Model-I for a formulation with $1 \mathrm{phr}$ of MBS and $4 \mathrm{phr}$ of sulfur that was cured isothermally at $330^{\circ} \mathrm{F}$.

The time evolution of the sulfurating species $A_{x}$ of different lengths is shown in Figure 16 . Since $A_{0}$ is the first sulfurating species formed, the time evolution of $A_{0}$ shows that it is formed at the onset of the vulcanization reaction with no induction period. As soon as $\mathrm{A}_{0}$ is formed, it can react with $S_{8}$ forming $A_{8}$; consequently, the time evolution of $A_{8}$ parallels $A_{0}$ and also exhibits little or no induction period. Although it is difficult to see from Figure 16, at very short times (i.e. less than $1 \mathrm{~min}$ ) the rate of production of $\mathrm{A}_{1}$ and $\mathrm{A}_{7}$ are identical as would be expected when sulfur is picked-up by MBTS as $S_{8}$ and then the inherent symmetry of the exchange reactions of the polysulfidic accelerator (i.e. R.27) produce equal amounts of $A_{1}$ and $A_{7}$ from $A_{8}$. However, as soon as $\mathrm{A}_{1}$ is formed, it is consumed by $\mathrm{S}_{8}$ to give $\mathrm{A}_{9}$, while $\mathrm{A}_{9}$ is not allowed to pick-up additional $S_{8}$ because of the model assumption that species with $x>16$ will not form. Examination of Figure 16 reveals that the longer length $A_{x}$ 's are formed prior to the shorter length $A_{x}$ 's. Specifically, the formation of $A_{x}$ with $x$ greater than 8 begins at around 0.4 minutes, reaching a maximum around $3.5 \mathrm{~min}$; in contrast for $\mathrm{A}_{\mathrm{x}}$ with $\mathrm{x}$ less than 8 , the formation begins at approximately $1 \mathrm{~min}$, reaching a maximum around $5 \mathrm{~min}$. The prediction that longer lengths of $A_{x}$ are formed prior to shorter lengths is a direct consequence of the $S_{8}$ sulfur insertion scheme of Model-I. The concentration profiles predicted by Model-I for the species $\mathrm{A}_{1}, \mathrm{~A}_{8}$ and $\mathrm{A}_{9}$ exhibit larger maximum concentrations than $\mathrm{A}_{\mathrm{x}}$ for other lengths. A high concentration of $\mathrm{A}_{8}$ results from an initially high concentration of $\mathrm{A}_{0}$, since it is the first sulfurating species formed. The concentration of $A_{1}$ which evolves according to Equation 7 is high because it represents the sum of the species BtS-S-SBt and BtS-Zn-SBt that are formed in the initial accelerator rearrangement reactions given by R.27 and during the desulfuration reactions of $\mathrm{Vu}_{\mathrm{x}}$ with $\mathrm{A}_{0}$ in the postcrosslinking phase given by R.60 and 61. The major contribution to $A_{1}$ comes from the desulfuration reactions; thus, the major production of $\mathrm{A}_{1}$ must wait until after the formation of $\mathrm{Vu}_{\mathrm{x}}$. As discussed in Section III, the contribution to $\mathrm{A}_{1}$ will most probably be BtS-S-SBt in the early stages by R.27, while the later stages will have contributions from BtS-S-Zn-SBt formed via R.60 and R.61; however, in the current analysis no distinction is made between zinc and zincfree species. 


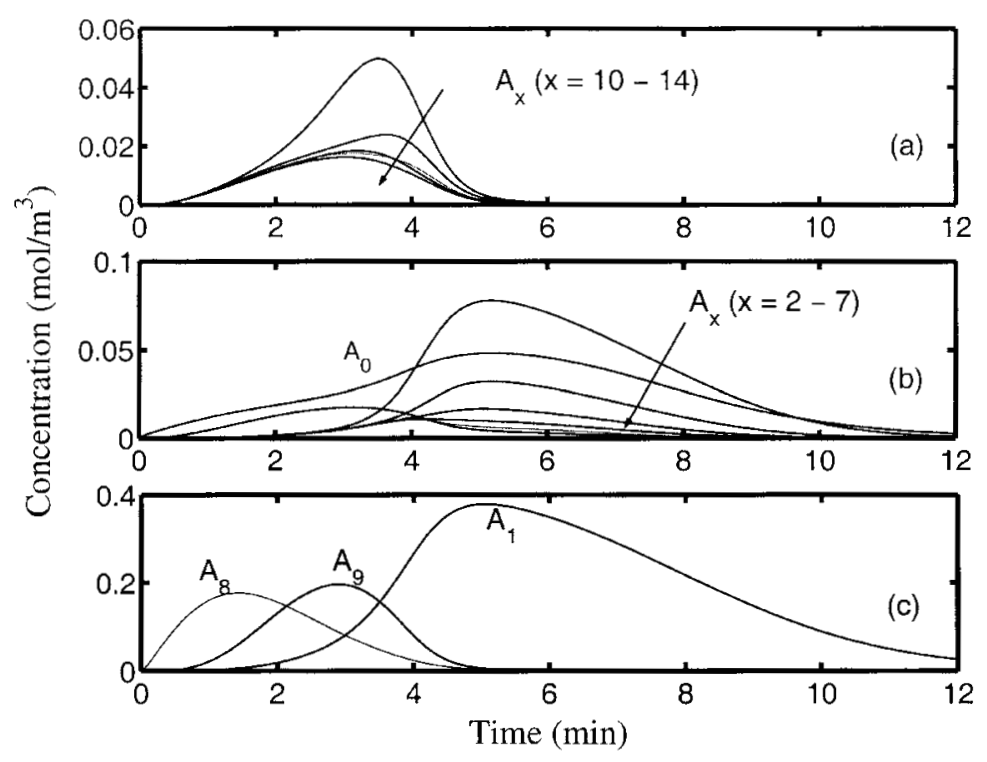

FIG. 16. - Concentration profiles predicted by Model-I for the different sulfurating species i.e. BtS-S $-\mathrm{SBt}$ and/or $\mathrm{BtS}-\mathrm{Zn}-\mathrm{S}_{\mathrm{x}}-\mathrm{SBt}$. Predictions are for a formulation with $1 \mathrm{phr}$ of MBS and $4 \mathrm{phr}$ or sulfur that was cured at $330^{\circ} \mathrm{F}$.

The relative fractions of mono, di and polysulfidic crosslinks at the maximum extent of crosslinking predicted by Model-I for the five different formulations cured at $330{ }^{\circ} \mathrm{F}$ are shown in Figure 17 as well as the total concentration of the crosslinks at the maximum cure. Comparing formulations $\mathrm{A}$ and $\mathrm{C}$, as the sulfur level is increased holding the accelerator concentration constant, the fraction of polysulfide crosslinks increases. And, comparing formulations $\mathrm{C}$ and $\mathrm{D}$, as the accelerator concentration is increased holding the sulfur concentration constant, the fraction of monosulfides increases. The increase in total number of crosslinks with increasing sulfur concentration is expected, since additional sulfur will introduce more opportunity for crosslink formation in the vulcanizate. The increase in the total number of crosslinks with increasing accelerator concentration is also intuitive. As the accelerator concentration increases the longer length crosslinks are redistributed to crosslinks of shorter length, resulting in an increase in the overall crosslink density even though the total amount of sulfur remains the same. Specifically, increasing the accelerator concentration results in more exchange reactions via R.27 among the sulfurating species in the accelerator chemistry and more desulfuration reactions in the post-crosslinking chemistry via R.60 and 61, with the result that a greater percentage of crosslinks of shorter length are formed. Predicting how the distribution of crosslink lengths is affected by the amount of sulfur and accelerator is necessary in order to predict how the overall crosslink density increases with both accelerator and sulfur. These results clearly indicate that the population balance method with its explicit accounting of the length distribution for the various chemical species is necessary to describe the important features of the vulcanization process. 


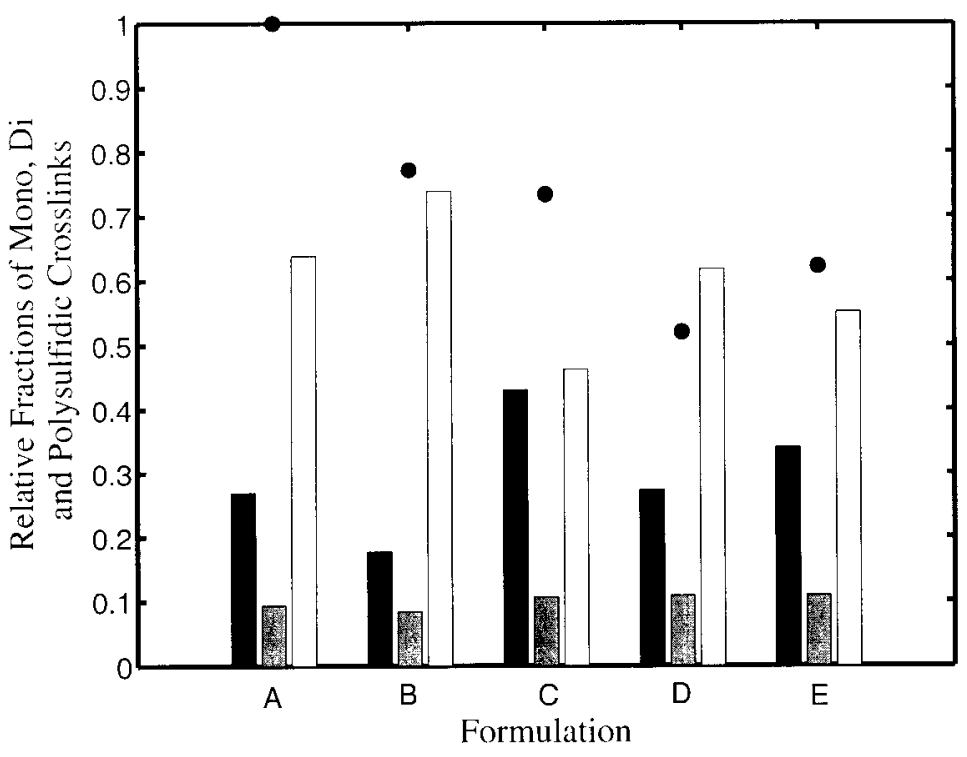

Fig. 17. - Relative percentages of mono $(\square)$, di $(\square)$ and polysulfidic $(\square)$ crosslinks for different formulations at maximum cure predicted by Model-I at $\mathrm{T}=330^{\circ} \mathrm{F}$. Formulation A: $1 \mathrm{phr}$ of MBS and $4 \mathrm{phr}$ of S, Formulation B: $0.5 \mathrm{phr}$ of MBS and $4 \mathrm{phr}$ of S, Formulation C: $1 \mathrm{phr}$ of MBS and $2 \mathrm{phr}$ of S,

Formulation D: $0.5 \mathrm{phr}$ of MBS and $2 \mathrm{phr}$ of S and Formulation E: $0.75 \mathrm{phr}$ of MBS and $2 \mathrm{phr}$ of S. The solid circles indicate the ratio of the total number of crosslinks at maximum cure relative to the total number of crosslinks at maximum cure for formulation $\mathrm{A}$, where the maximum number of crosslinks for formulation $\mathrm{A}$ is $83 \mathrm{~mol} / \mathrm{m}^{3}$.

In Figures 18 and 19, the predictions of Model-I for evolution of the total concentration of crosslinks are compared with experimental ODR data at 310 and $298{ }^{\circ} \mathrm{F}$ for the same five formulations presented previously. The predictions are again reasonable, describing the correct order of the ODR response as a function of composition. The effect of temperature on the vulcanization kinetics for a single formulation is shown in Figure 20. In agreement with the experimental data, Model-I predicts a decrease in the final modulus with an increase in temperature. This occurs because the temperature preferentially accelerates degradation reactions, like the formation of elastically ineffective loops, resulting in the loss of crosslinks and hence a lower modulus. 


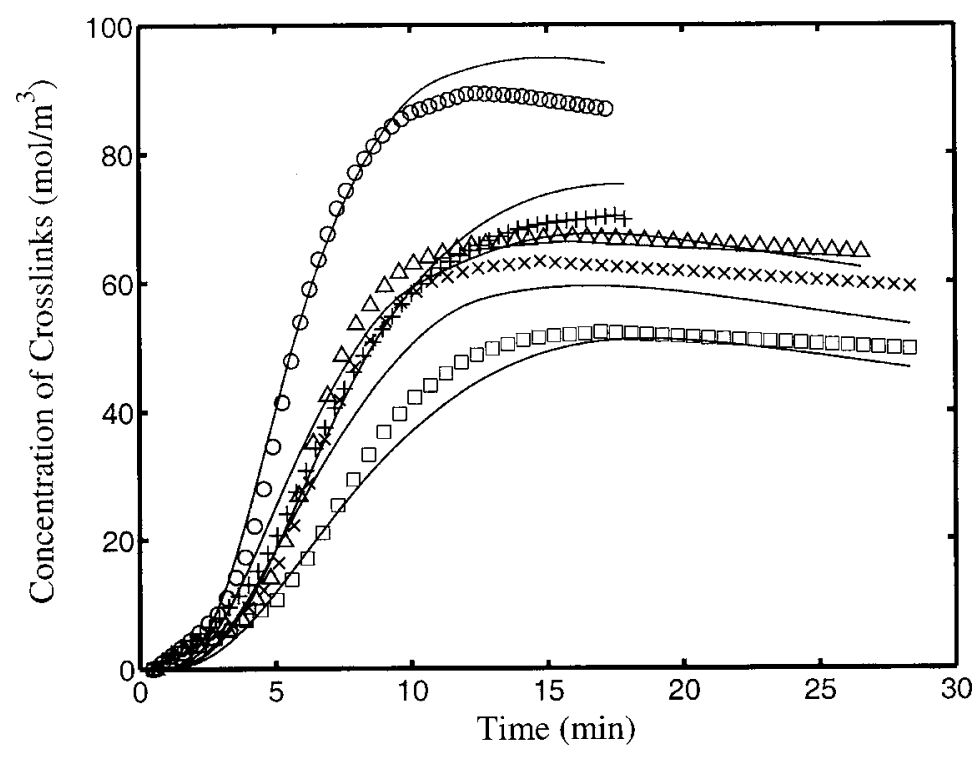

Fig. 18. - Comparison of the temporal evolution of the total concentration of crosslinks predicted by Model-I with experimental ODR data for different formulations cured at $310^{\circ} \mathrm{F}$. Formulations include: (o) $1 \mathrm{phr} \mathrm{MBS}$ and $4 \mathrm{phr} \mathrm{S}$; (+) 1 phr MBS and $2 \mathrm{phr} \mathrm{S}$; $(\Delta) 0.5 \mathrm{phr}$ MBS and $4 \mathrm{phr} \mathrm{S}$; (x) $0.75 \mathrm{phr}$ MBS and $2 \mathrm{phr} \mathrm{S}$; $(\square) 0.5 \mathrm{phr}$ MBS and $2 \mathrm{phr}$ S. The predictions are indicated by the solid lines. No retarder is used and the rest of the cure-system is given in Section 6.

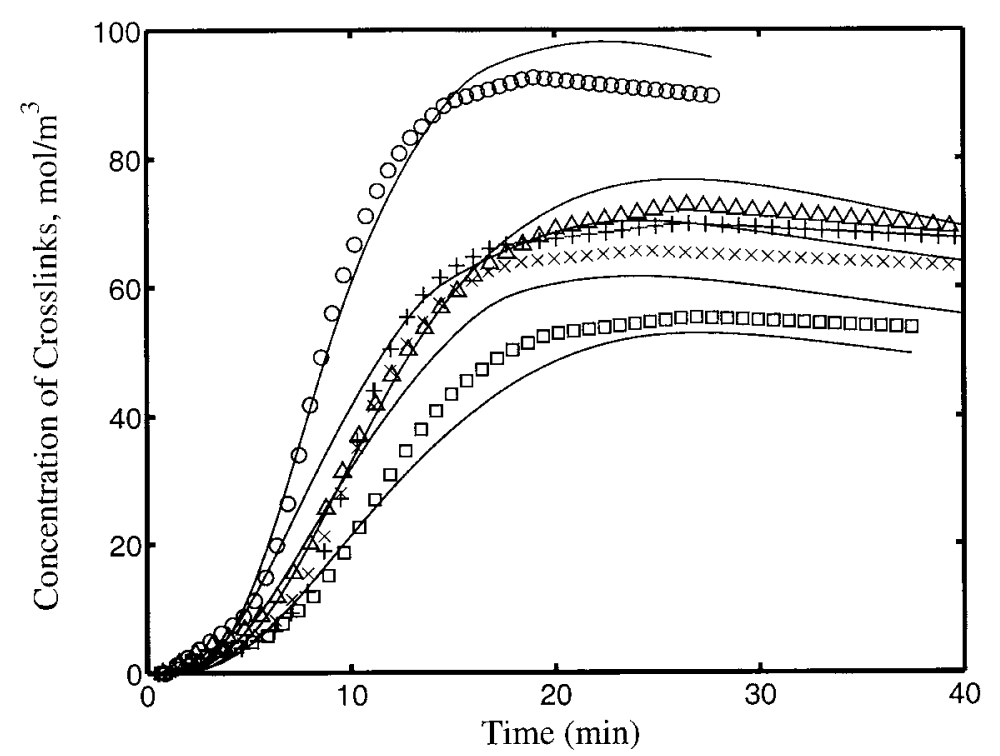

Fig. 19. - Comparison of the temporal evolution of the total concentration of crosslinks predicted by Model-I with experimental ODR data for different formulations cured at $298^{\circ} \mathrm{F}$. Formulations include: (o) 1 phr MBS and $4 \mathrm{phr} \mathrm{S}$; (+) 1 phr MBS and $2 \mathrm{phr} \mathrm{S;}(\Delta) 0.5 \mathrm{phr}$ MBS and $4 \mathrm{phr} \mathrm{S}$;

(x) $0.75 \mathrm{phr}$ MBS and $2 \mathrm{phr} \mathrm{S}$; $\square$ ) $0.5 \mathrm{phr}$ MBS and $2 \mathrm{phr}$ S. The predictions are indicated by the solid lines. No retarder is used and the rest of the cure-system is given in Section 6. 


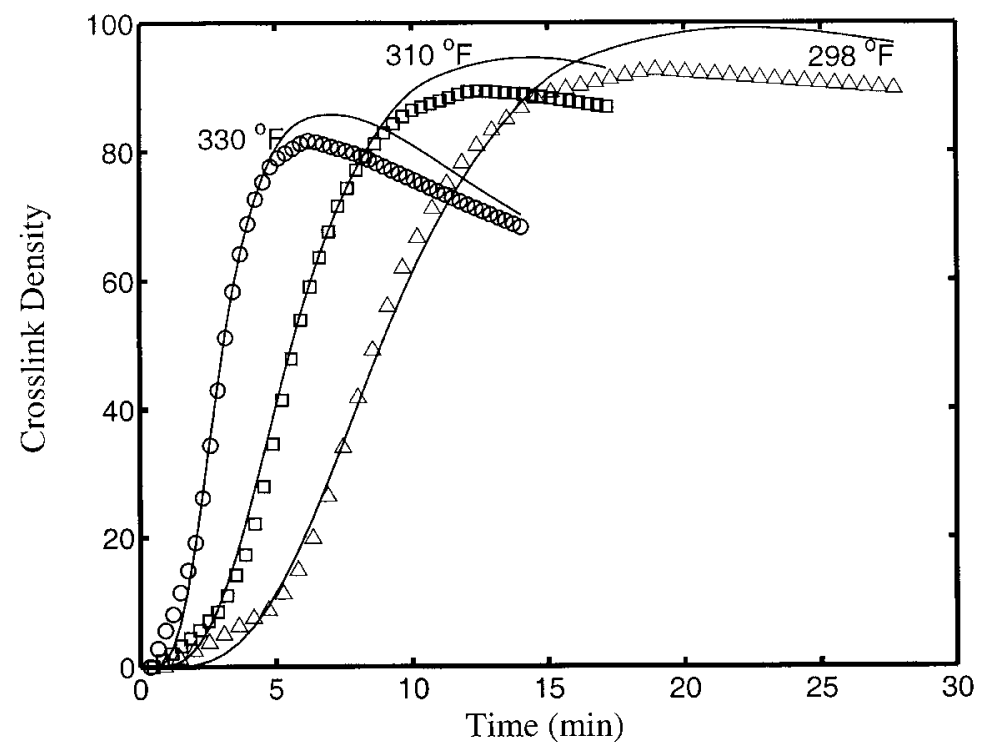

FIG. 20. - Comparison of the temporal evolution of the crosslink density using Model-I for a formulation with $1 \mathrm{phr}$ MBS accelerator and $4 \mathrm{phr} \mathrm{S}$ cured at three different temperatures. Curing temperatures include:

(o) $330^{\circ} \mathrm{F},(\square) 310^{\circ} \mathrm{F}$ and $(\Delta) 298^{\circ} \mathrm{F}$. The predictions are indicated by the solid lines.

Increasing temperature leads to lowering of the crosslink density.

The PBEs for Model-I were able to predict most of the features of the ODR data for MBS accelerated sulfur vulcanization, including scorch delay, crosslinking and reversion for formulations with various initial sulfur and accelerator concentrations; moreover, Model-I was also able to capture the increase in the crosslink density with increasing sulfur concentration and the increase in the maximum crosslink density with increasing amount of accelerator. The key feature of Model-I that enabled a reasonably accurate description of the observed ODR data was the explicit incorporation of the polysulfidic nature of all species. The kinetic parameters in the PBEs were fit to the temporal evolution of the total crosslink density as determined via the ODR experiments, and the temporal evolution of the distributions of the intermediate species were predictions of the PBEs. Although there is no direct experimental measurement of the intermediate species like $\mathrm{A}_{\mathrm{x}}, \mathrm{B}_{\mathrm{x}}$ and $\mathrm{B}_{\mathrm{x}}{ }_{\mathrm{x}}$ and of the distribution of crosslinks for the MBS-natural rubber system being studied, Gradwell, et al. ${ }^{46}$ and Morgan, and McGill ${ }^{60}$ did measure the distribution of $A_{x}$ species upon reaction of MBTS with sulfur when no rubber is present. They observed that the smaller lengths of $A_{x}$ are more abundant and are formed first in contrast to the predictions of Model-I as shown in Figure 16. This indicates that there is a serious problem with Model-I even though it is able to describe the ODR data. This shortcoming of Model-I is a consequence of the assumption that sulfur is picked-up by the accelerator as $S_{8}$ and will be addressed in the following Section.

\section{B. PREDICTIONS FOR MODEL II: SEQUENTIAL SULFUR PICK-UP}

The results presented in the previous Section were obtained using Model-I, where sulfur activation was assumed to occur by the addition of $\mathrm{S}_{8}$ units to the accelerator. In Model-II, the sulfur activation is assumed to be sequential and the kinetics are defined by Equations 12-14, 17, $18,25,26$ and 27-39, where the length of the polysulfidic chains is again assumed to be less than 16. These 107 nonlinear, ordinary differential equations contain nine rate constants, which are determined by fitting the ODR data using the Levenberg-Marquardt algorithm as discussed in Section VII.A. The Model-II rate constants that were regressed from the ODR data are given in 
Table VIII along with the associated activation energies. The predictions for Model-II are shown in Figure 21 along with the experimental ODR data for the same five formulations shown previously in Figure 13. The predictions for Model-II show the same behavior in the evolution of the total number of crosslinks as observed previously with Model-I, but the predictions for ModelII are improved as compared to Model-I; specifically, the RMS error has decreased by $30 \%$ from 51.6 to 35.4. More important than the decrease in the RMS error is the qualitative improvement in certain aspects of the predictions. Specifically, both the shape of the cure curves at the maximum and the slope of the response at long times are noticeably better. In Figure 22, the evolution of the concentrations for the lumped species, A, B, B* Vu, MBS and sulfur are shown for Model-II. Although, most aspects of the profiles shown in Figure 22 are qualitatively similar to Figure 14, the predicted concentration profile for the depletion of sulfur is different for ModelII. In Figure 14 the sulfur consumption for Model-I shows a smooth decrease, while for ModelII the sulfur consumption shows an initial plateau followed by a rapid depletion. In addition, the concentration profiles predicted by Model-II for B and $\mathrm{B}^{*}$ are sharper and occur somewhat earlier than the predictions of Model-I shown in Figure 14.

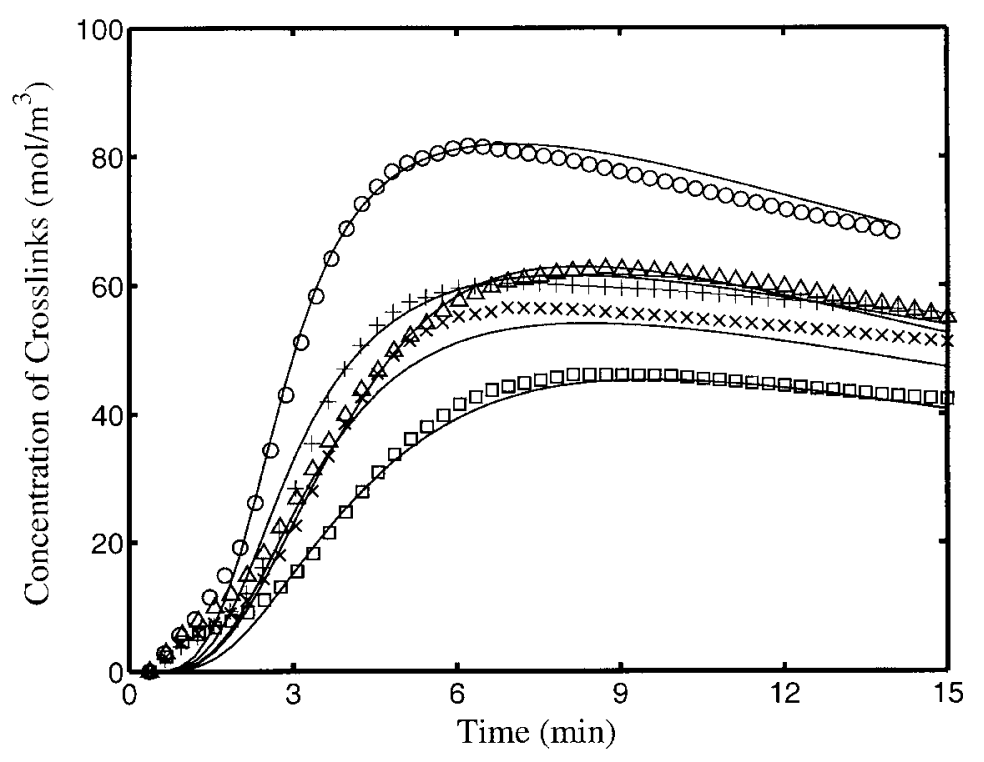

FIG. 21. - Comparison of the temporal evolution of the total (i.e. sum of all lengths) concentration of crosslinks predicted by Model-II with experimental ODR data for different formulations cured at $330^{\circ} \mathrm{F}$.

Formulations include: (o) $1 \mathrm{phr}$ MBS and $4 \mathrm{phr} \mathrm{S}$; (+) $1 \mathrm{phr}$ MBS and $2 \mathrm{phr} \mathrm{S} ;(\Delta) 0.5 \mathrm{phr}$ MBS and $4 \mathrm{phr} \mathrm{S}$;

(x) $0.75 \mathrm{phr}$ MBS and $2 \mathrm{phr} \mathrm{S}$; $(\square) 0.5 \mathrm{phr}$ MBS and $2 \mathrm{phr}$ S. The predictions are indicated by the solid lines No retarder is used and the rest of the cure-system is given in Section 6. 
TABLE VIII

Activation Energy and PRe-EXPonential Factors For Model-II

\begin{tabular}{ccc}
\hline Rate-Constant & Pre-Exponential Factor & Activation Energy (kcal/mol) \\
\hline $\mathrm{k}_{\mathrm{MBS}}$ & $3.104 \times 10^{17}$ & 37.4 \\
$\mathrm{k}_{\mathrm{MBS}-\mathrm{MBT}}$ & $1.038 \times 10^{4}$ & 7.3 \\
$\mathrm{k}_{\mathrm{A}-\mathrm{S}}$ & $2.311 \times 10^{15}$ & 30.8 \\
$\mathrm{k}_{\mathrm{A}-\mathrm{A}}$ & $3.443 \times 10^{19}$ & 41.7 \\
$\mathrm{k}_{\mathrm{A}-\mathrm{R}}$ & $1.484 \times 10^{14}$ & 26.9 \\
$\mathrm{k}_{\mathrm{B}-\mathrm{R}}$ & $2.322 \times 10^{6}$ & 13.9 \\
$\mathrm{k}_{\mathrm{VU}}$ & $3.425 \times 10^{4}$ & 6.5 \\
$\mathrm{k}_{\mathrm{DEG}}$ & $1.801 \times 10^{16}$ & 35.1 \\
$\mathrm{k}_{\mathrm{E}-\mathrm{S}}$ & $4.825 \times 10^{3}$ & 6.9 \\
\hline
\end{tabular}

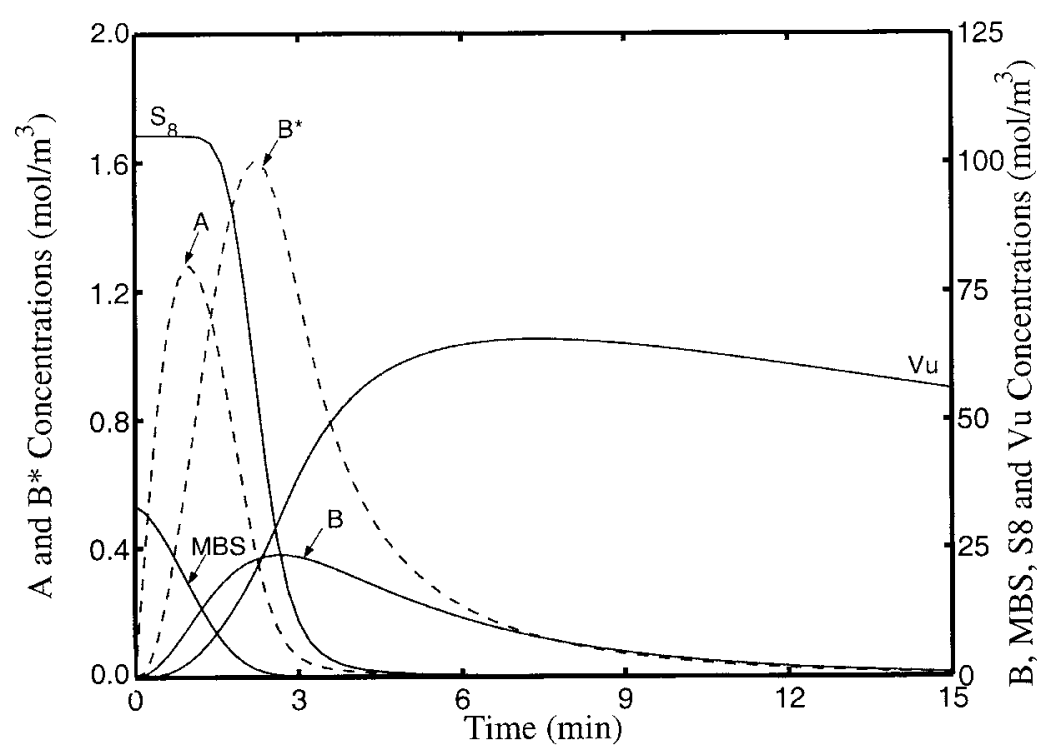

FIG. 22. - Temporal evolution of lumped species A, B, B*, Vu, MBS and Sulfur $\left(\mathrm{S}_{8}\right)$ for the $(1,4)$ formulation, cured isothermally at $\mathrm{T}=330 \mathrm{~F}$ (Model-II). Here $\mathrm{A}=\sum_{x=0}^{14} A_{x}, \mathrm{~B}=\sum_{x=1}^{16} B_{x}, \mathrm{~B}^{*}=\sum_{x=1}^{16} B_{x}^{*}$ and $\mathrm{Vu}=\sum_{x=0}^{16} V u_{x}$.

The evolution of the crosslinks for different polysulfidic lengths is shown in Figure 23. The concentration of longer length crosslinks initially grows which is followed by a decline owing to desulfuration and degradation reactions. Contrasting the predictions of Model-II in Figure 23 with the predictions for Model-I shown in Figure 15, in Model-I the longer length crosslinks are formed prior to shorter length crosslinks, while in Model-II the different length crosslinks are formed at approximately the same time. The concentration profiles for the various sulfurating species predicted by Model-II are shown in Figure 24, where there are some striking differences as compared to the predictions for Model-I shown in Figure 16. In Model-I the species $A_{1}, A_{8}$ and $\mathrm{A}_{9}$ dominate in comparison to the other $\mathrm{A}_{\mathrm{x}}$ species, but in Model-II the concentration profiles are systematically ordered, where there is a gradual decrease in the $\mathrm{A}_{\mathrm{x}}$ concentration pro- 
files with increasing polysulfidic length. Further, the shorter length $A_{x}$ species are now formed prior to the corresponding longer length species. The trend predicted by Model-II of a decrease in the sulfurating species $\mathrm{A}_{\mathrm{x}}$ with increasing $\mathrm{x}$ qualitatively agrees with the experimental studies of Gradwell et al. ${ }^{46}$ Campbell and Wise, ${ }^{22,23}$ Sullivan et al. ${ }^{136}$ and more recently by Morgan and McGill. ${ }^{137}$ The origin of the initial plateau in the depletion profile for sulfur predicted for ModelII shown in Figure 22 can now be addressed. Since Model-II proceeds by sequential sulfur activation, for every mole of $S_{x}$ that is consumed, a mole of $S_{x-1}$ is formed; thus, the total number of moles of free sulfur is constant during the early part of the vulcanization process resulting in the initial plateau. The total number of moles of free sulfur will, however, begin to decrease when the sequential sulfur reaches the stage that $S_{1}$ is produced. The predicted evolution of the various $\mathrm{S}_{\mathrm{x}}$ species is shown in Figure 25 for Model-II. While concentration of $\mathrm{S}_{8}$ decreases monotonically, the concentration of the other $S_{x}$ species first increases and then decreases, eventually approaching to zero. The initial plateau in the molar concentration of sulfur followed by a rapid decrease predicted by Model-II agrees with the experimental observation of Sullivan et al. ${ }^{136}$

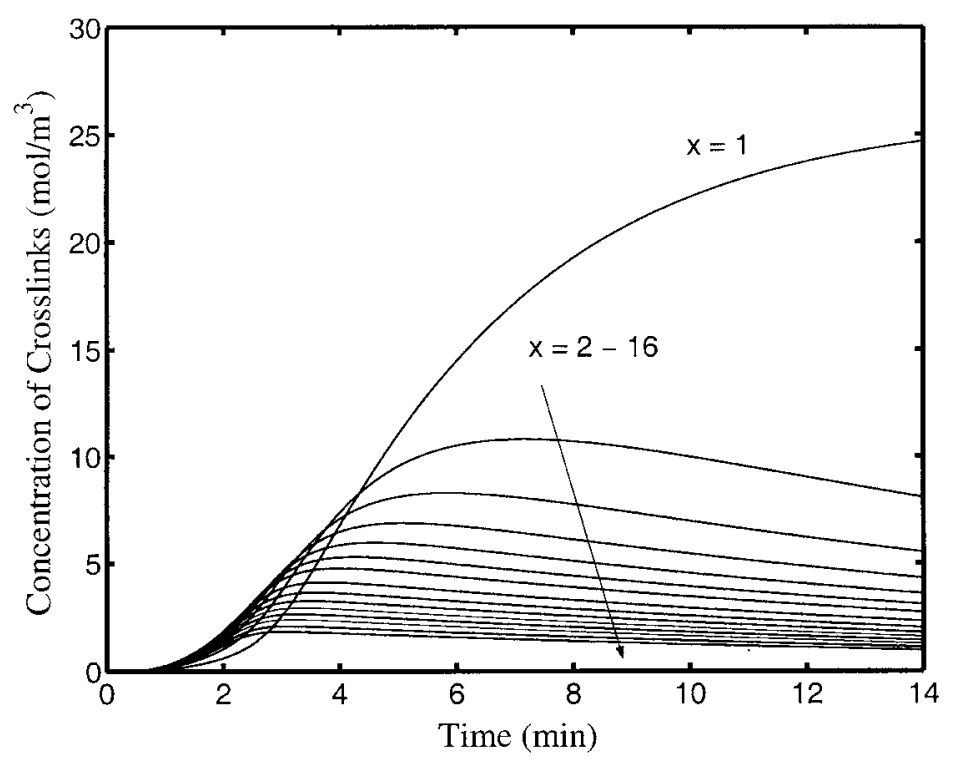

FIG. 23. - Temporal evolution of crosslinks of different lengths predicted by Model-II for a formulation with $1 \mathrm{phr}$ of MBS and $4 \mathrm{phr}$ of sulfur that was cured isothermally at $330^{\circ} \mathrm{F}$. 


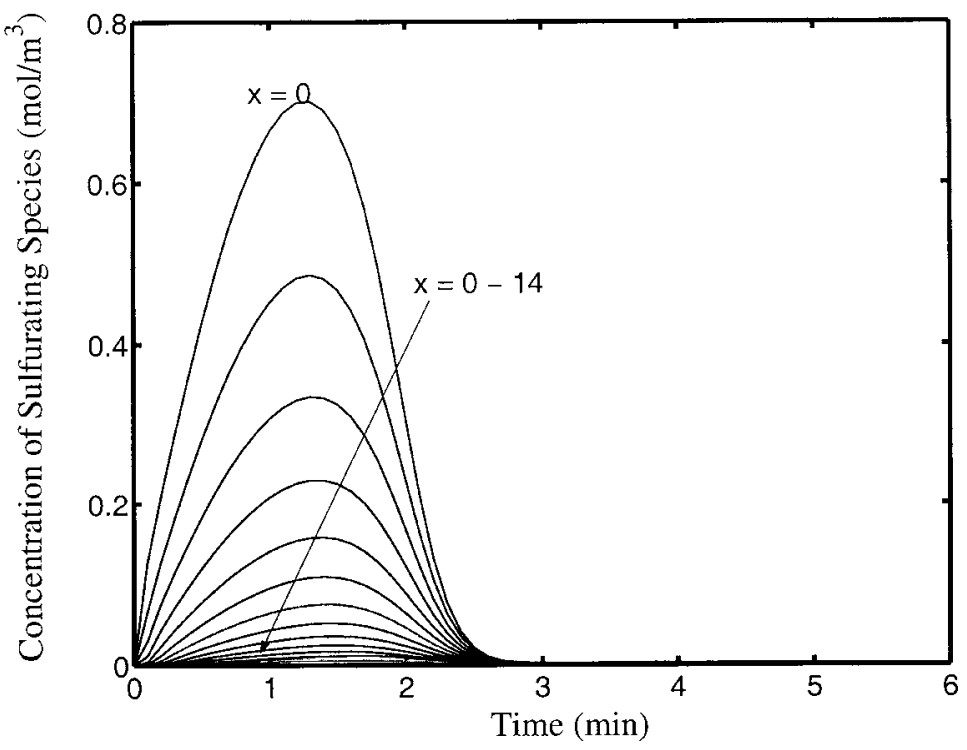

FIG. 24. - Concentration profiles predicted by Model-II for the different sulfurating species i.e. BtS-S $\mathrm{x}_{\mathrm{x}} \mathrm{SBt}$ and/or $\mathrm{BtS}-\mathrm{Zn}-\mathrm{S}_{\mathrm{x}}-\mathrm{SBt}$. Predictions are for a formulation with $1 \mathrm{phr}$ of MBS and $1 \mathrm{phr}$ or sulfur that was cured at $330^{\circ} \mathrm{F}$.

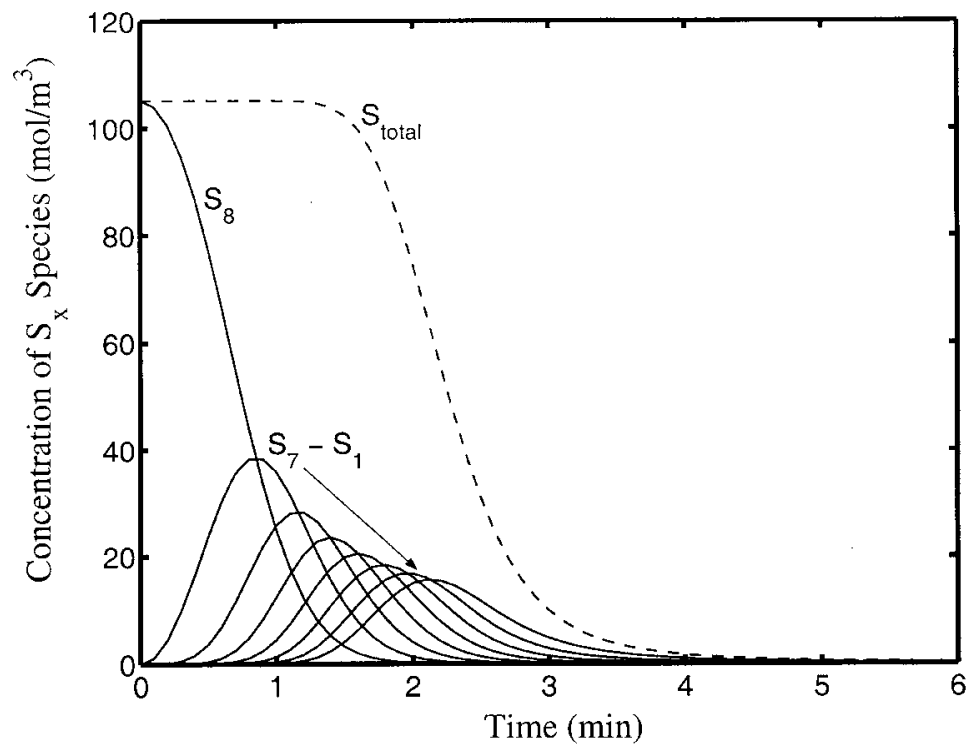

FIG. 25. - Temporal evolution of $\mathrm{S}_{\mathrm{x}}$ of different lengths predicted by Model-II for a formulation with $1 \mathrm{phr}$ MBS and $4 \mathrm{phr} \mathrm{S}$ that was cured isothermally at $\mathrm{T}=330^{\circ} \mathrm{F} . \quad S_{\text {TOTAL }}=\sum_{x=1}^{8} S_{x}$

The relative amounts of mono, di and polysulfidic crosslinks and the maximum extent of cure as predicted by Model-II are shown in Figure 26. Analogous to Model-I, Model-II also predicts a higher concentration of polysulfidic crosslinks compared to mono and disulfidic crosslinks for formulations with high sulfur to accelerator ratios for reasons identical to those already discussed in Section VII.A. In Figures 27 and 28 the predictions of Model-II are com- 
pared with the isothermal cure data at 310 and $298^{\circ} \mathrm{F}$. Comparing the predictions at the lowest vulcanization temperature of $298^{\circ} \mathrm{F}$ for Model-I in Figure 19 versus the corresponding predictions for Model-II in Figure 28, the predictions for Model-II at the lower temperature are significantly better than the predictions for Model-I. The effect of temperature on the vulcanization kinetics is shown in Figure 29, where the Model-II predictions are improved significantly as compared to the predictions of Model-I shown in Figure 20. Just as was the case for Model-I, increasing the temperature leads to an increased rate of loop formation, and hence, a lower concentration of crosslinks. The predictions for Model-II as shown in Figures 21 through 30 for a variety of formulations exhibit both (i) a better fit of the data as compared to Model-I, and (ii) the correct qualitative trend for the growth of the $A_{x}$ sulfurating species as shown in Figure 24 . Because of these reasons, we believe that Model-II is a more appropriate description of the vulcanization kinetics for benzothiazole accelerated sulfur vulcanization.

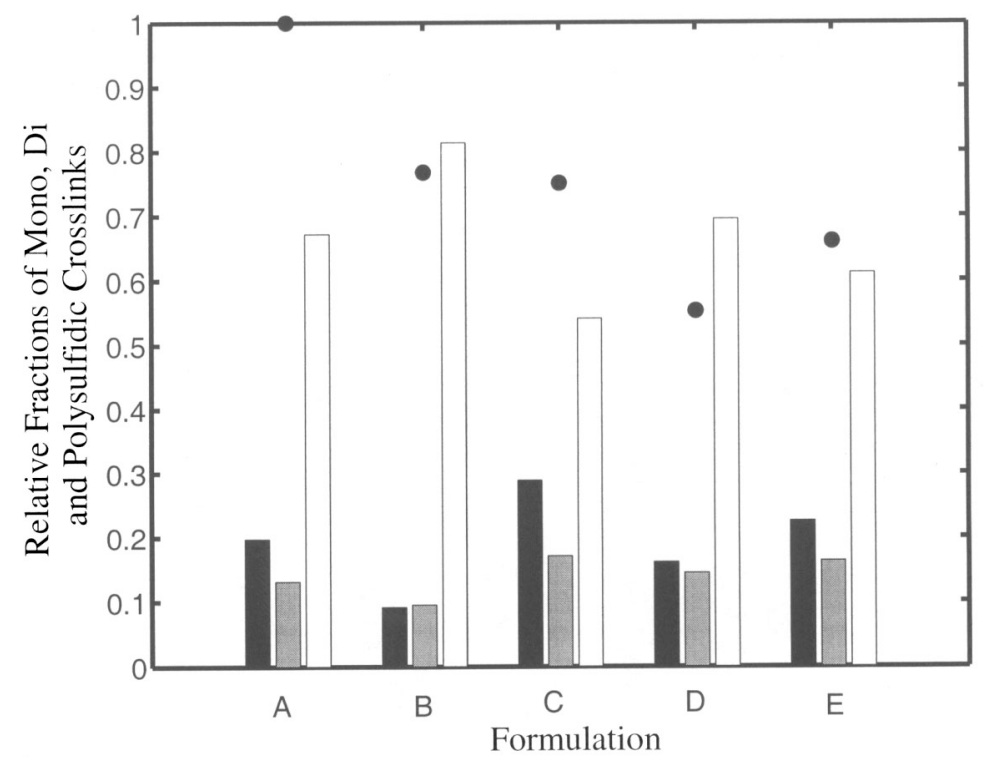

FIG. 26. — Relative percentages of mono ( $\mathbf{\square})$, di ( $\square$ ) and polysulfidic ( $\square$ ) crosslinks for different formulations at maximum cure predicted by Model-II at T $=330^{\circ} \mathrm{F}$. Formulation A: $1 \mathrm{phr}$ of MBS and $4 \mathrm{phr}$ of S; Formulation B: 0.5 phr of MBS and 4 phr of S; Formulation C: 1 phr of MBS and 2 phr of S;

Formulation D: $0.5 \mathrm{phr}$ of MBS and $2 \mathrm{phr}$ of S; and Formulation E: $0.75 \mathrm{phr}$ of MBS and $2 \mathrm{phr}$ of S.

The solid circles indicate the ratio of the total number of crosslinks at maximum cure relative to the total number of crosslinks at maximum cure for formulation $\mathrm{A}$, where the maximum number of crosslinks for formulation $\mathrm{A}$ is $83 \mathrm{~mol} / \mathrm{m}^{3}$. 


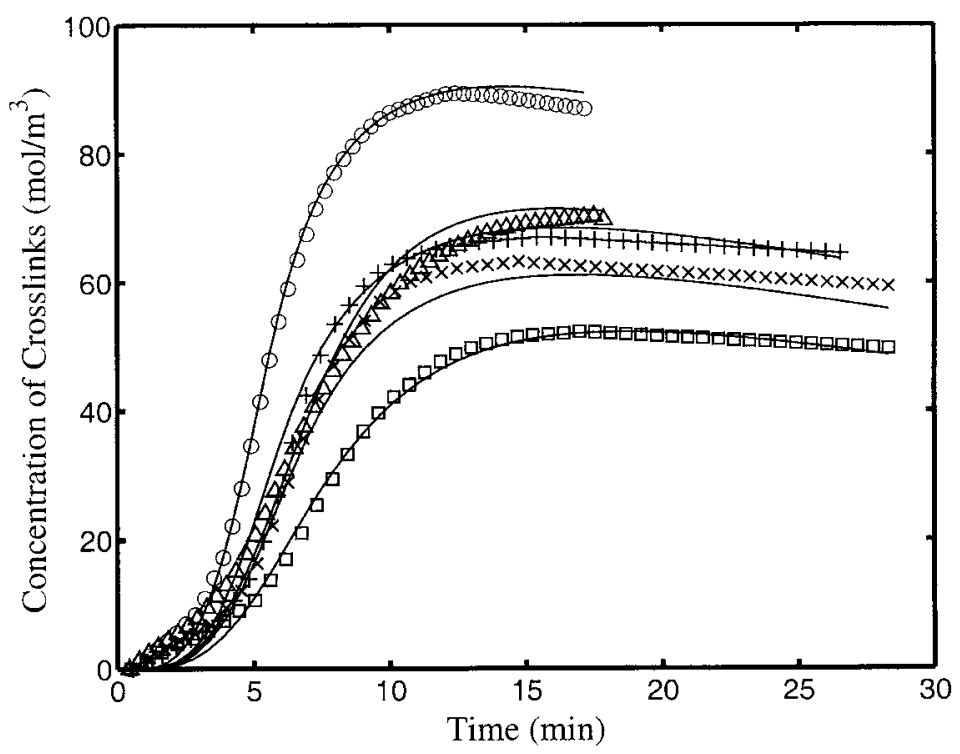

FIG 27. - Comparison of the temporal evolution of the total (i.e. sum of all lengths) concentration of crosslinks predicted by Model-II with experimental ODR data for different formulations cured at $310^{\circ} \mathrm{F}$. Formulations include:

(o) $1 \mathrm{phr}$ MBS and $4 \mathrm{phr} \mathrm{S}$; (+) $1 \mathrm{phr}$ MBS and $2 \mathrm{phr} \mathrm{S}$; $(\Delta) 0.5 \mathrm{phr}$ MBS and $4 \mathrm{phr}$;

(x) $0.75 \mathrm{phr}$ MBS and $2 \mathrm{phr} \mathrm{S}$; ( $\square$ ) $0.5 \mathrm{phr}$ MBS and $2 \mathrm{phr} \mathrm{S}$. The predictions are indicated by the solid lines. No retarder is used and the rest of the cure-system is given in Section 6.

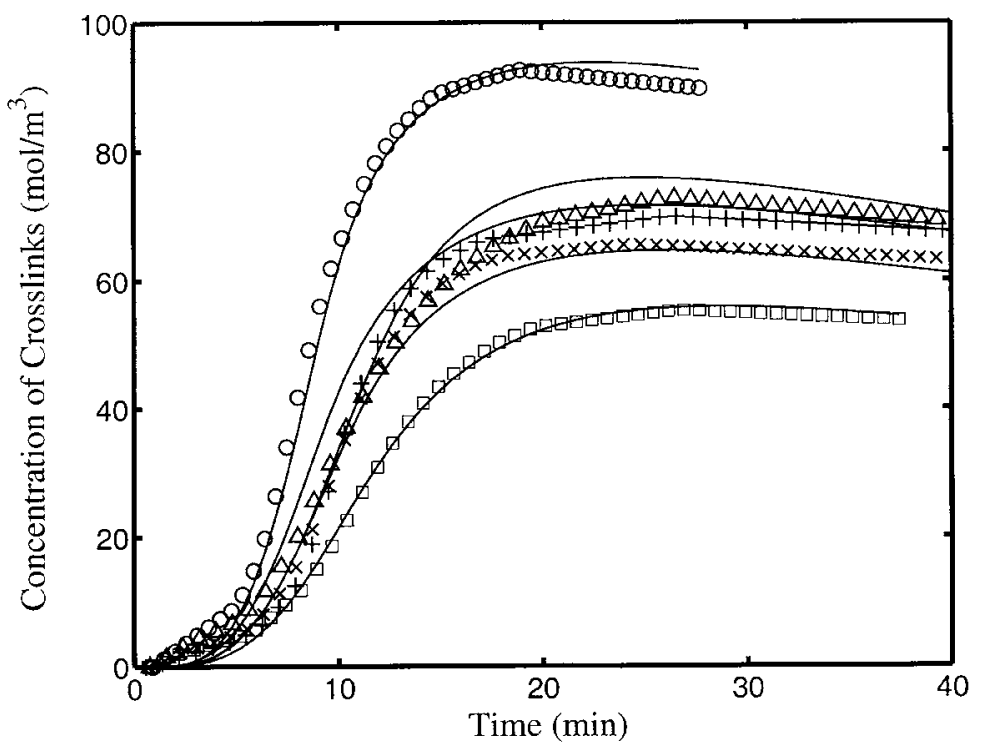

FIG. 28. - Comparison of the temporal evolution of the total (i.e. sum of all lengths) concentration of crosslinks predicted by Model-II with experimental ODR data for different formulations cured at $298^{\circ} \mathrm{F}$. Formulations include:

(o) $1 \mathrm{phr}$ MBS and $4 \mathrm{phr} \mathrm{S}$; (+) 1 phr MBS and $2 \mathrm{phr} \mathrm{S}$; $(\Delta) 0.5 \mathrm{phr}$ MBS and $4 \mathrm{phr} \mathrm{S}$;

(x) $0.75 \mathrm{phr}$ MBS and $2 \mathrm{phr} \mathrm{S}$; $(\square) 0.5 \mathrm{phr}$ MBS and $2 \mathrm{phr}$ S. The predictions are indicated

by the solid lines. No retarder is used and the rest of the cure-system is given in Section VI. 


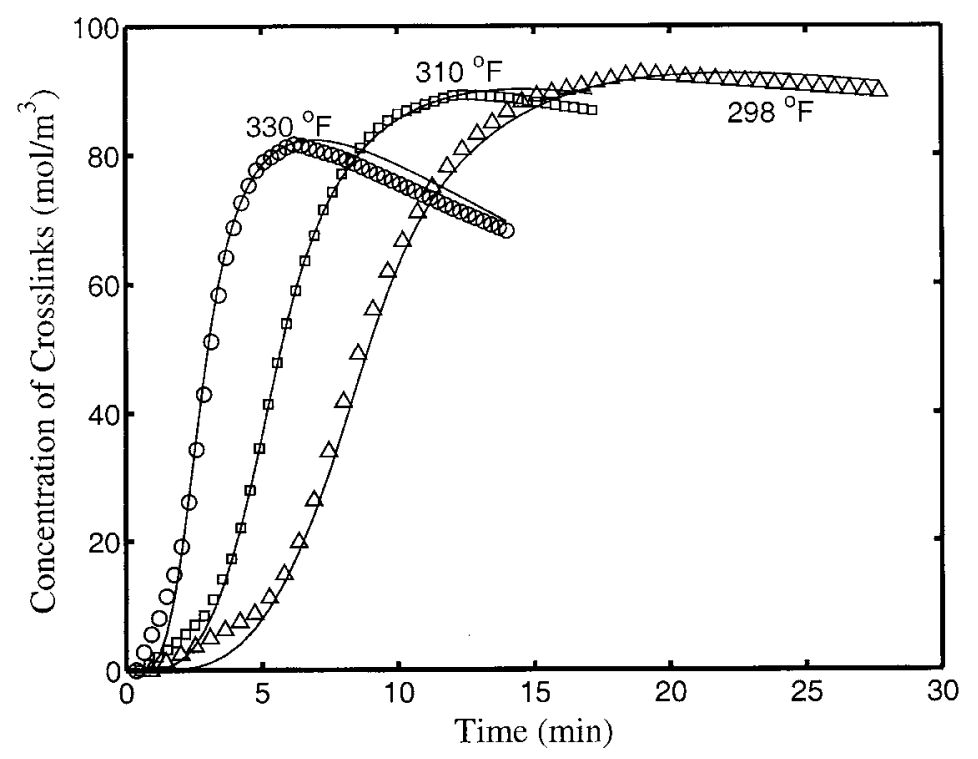

FIG. 29. - Comparison of the temporal evolution of the crosslink density using Model-II for a formulation with $1 \mathrm{phr}$ MBS accelerator and $4 \mathrm{phr}$ S cured at three different temperatures. Curing temperatures include: (o) $330^{\circ} \mathrm{F},(\square) 310^{\circ} \mathrm{F}$ and $(\Delta) 298^{\circ} \mathrm{F}$. The predictions are indicated by the solid lines. Increasing temperature leads to lowering of the crosslink density.

In Figure 30, the predictions for Model-II are shown for vulcanization systems with a CTP retarder at $310^{\circ} \mathrm{F}$ and $330^{\circ} \mathrm{F}$. The two rate constants associated with the retarder chemistry (i.e. $\mathrm{k}_{\mathrm{RET}}$ and $\mathrm{k}_{\mathrm{CDB}}$ ) were determined by optimization with the ODR data set containing retarders, where all the rate constants that had been determined for the retarder-free systems were set to those previously determined values. The values for the two retarder rate constants are $\mathrm{k}_{\mathrm{RET}}=$ $4.15 \times 10^{5} \mathrm{~m}^{3} / \mathrm{mol} \cdot \mathrm{min}$ and $\mathrm{k}_{\mathrm{CDB}}=4.19 \times 10^{27} \mathrm{~m}^{3} / \mathrm{mol} \cdot \mathrm{min}$ with associated activation energies of $9.9 \mathrm{kcal} / \mathrm{mol}$ and $53.0 \mathrm{kcal} / \mathrm{mol}$, respectively. In agreement with the ODR data, the model captures the increase in the scorch delay with increasing retarder concentration and where the retarder only minimally affects the maximum value of the modulus. The effect of retarder on the predictions for Model-I and Model-II are similar, thus, only the predictions for Model-II have been shown. 


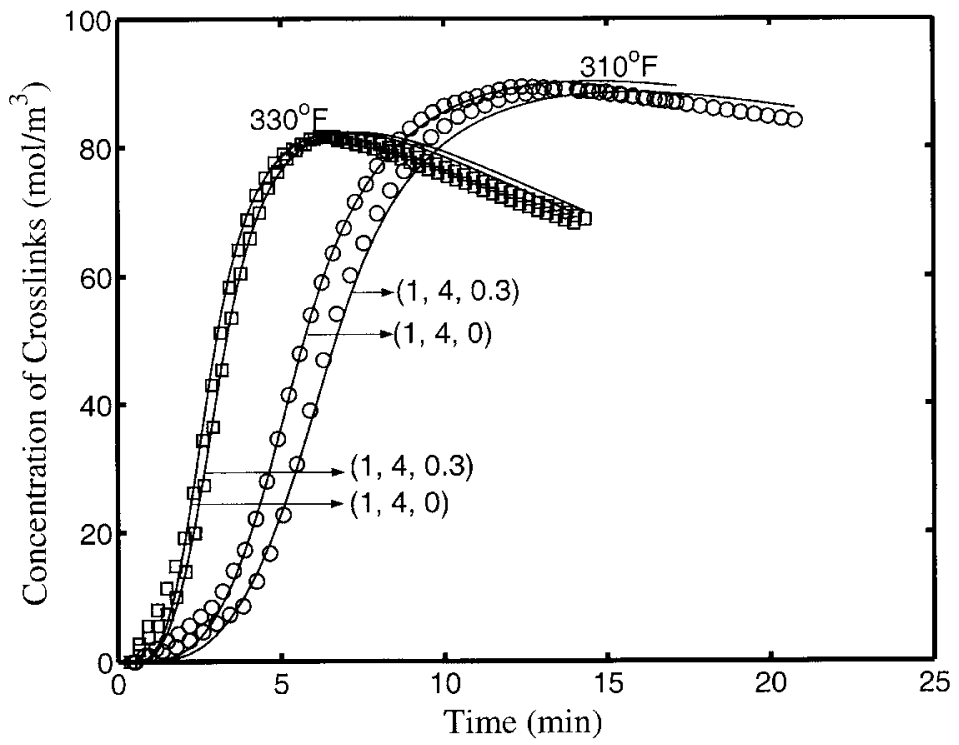

FIG. 30. - Comparison of the temporal evolution of the crosslink density using Model-II for a formulation with $1 \mathrm{phr}$ MBS accelerator, $4 \mathrm{phr} S$ and 0 and $0.3 \mathrm{phr}$ CTP cured at two different temperatures, i.e. $330^{\circ} \mathrm{F}$ and $310^{\circ} \mathrm{F}$. The predictions are indicated by solid lines.

We will now develop Model-III, which is a subtle variant of Model-II where the difference is in the details of the mechanism of sulfur activation. In Model-II it was assumed that BtSS $\mathrm{z} S \mathrm{St}$ picks-up sulfur by a concerted mechanism as given in R.20. In Model-III we will assume that $\mathrm{BtSS}_{\mathrm{z}} \mathrm{SBt}$ first decomposes into $\mathrm{BtSS}_{\mathrm{z}}{ }^{\cdot}$ radicals, which subsequently cause sulfur activation; specifically,

$$
\begin{aligned}
& \text { BtS-S }- \text {-SBt } \stackrel{\mathrm{k}_{\mathrm{B}}}{\longrightarrow} \text { BtS-S } \mathrm{y}_{\mathrm{y}}+\mathrm{BtS}_{\mathrm{z}} \cdot \quad(\mathrm{x}=\mathrm{y}+\mathrm{z})
\end{aligned}
$$

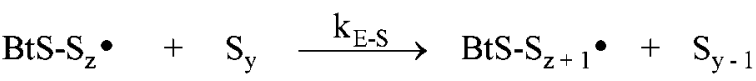

$$
\begin{aligned}
& \mathrm{BtS}-\mathrm{S}_{\mathrm{y}} \cdot+\mathrm{BtS}-\mathrm{S}_{\mathrm{z}} \bullet \stackrel{\mathrm{k}_{\mathrm{E}-\mathrm{E}}}{\longrightarrow} \mathrm{BtS}-\mathrm{S}_{\mathrm{y}+\mathrm{z}}-\mathrm{SBt} \\
& \mathrm{BtS}-\mathrm{SBt}+\mathrm{BtS}-\mathrm{S}_{\mathrm{y}}-\mathrm{SBt} \stackrel{\mathrm{k}_{\mathrm{A}-\mathrm{A}}}{\longrightarrow} \mathrm{BtS}-\mathrm{S}_{\mathrm{x}}-\mathrm{SBt}+\mathrm{BtS}-\mathrm{S}_{\mathrm{z}}-\mathrm{SBt} \quad(\mathrm{y}=\mathrm{x}+\mathrm{z})
\end{aligned}
$$

The sulfur activation scheme in R.80 is a slightly more detailed scheme than Model-II, although the number of rate constants is the same, since a number of the above reaction mechanisms have already been employed in other parts of the PBEs for Model-II. The reaction scheme for ModelIII is summarized in Table IX, where the resulting PBEs are only slightly changed from those developed in Section IV.B for Model-II. The rate constants for Model-III were regressed from the same set of ODR data by the Levenberg-Marquadt algorithm, ${ }^{135}$ and the rate constants are reported in Table X. The predictions of Model-III for the evolution of the total crosslink density as compared to the experimental ODR data are shown in Figures 31 through 33, where ModelIII exhibits slightly better quantitative agreement with the experimental data as compared to Model-II; specifically there is a $14 \%$ reduction in the RMS error. 


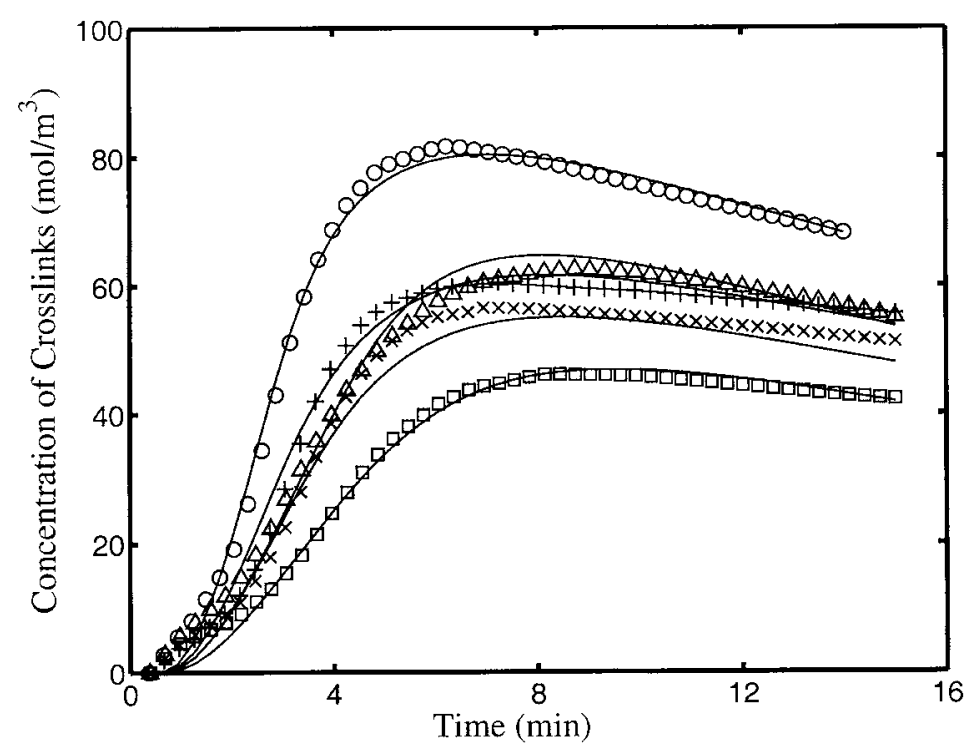

FIG. 31. - Comparison of the temporal evolution of the total (i.e. sum of all lengths) concentration of crosslinks predicted by Model-III with experimental ODR data for different formulations cured at $330^{\circ} \mathrm{F}$.

Formulations include: (o) $1 \mathrm{phr}$ MBS and $4 \mathrm{phr} \mathrm{S}$; (+) 1 phr MBS and $2 \mathrm{phr} \mathrm{S;}(\Delta) 0.5 \mathrm{phr}$ MBS and $4 \mathrm{phr} \mathrm{S}$; (x) $0.75 \mathrm{phr}$ MBS and $2 \mathrm{phr} \mathrm{S}$; ( $\square$ ) $0.5 \mathrm{phr}$ MBS and $2 \mathrm{phr}$ S. The predictions are indicated by the solid lines. No retarder is used and the rest of the cure-system is given in Section VI.

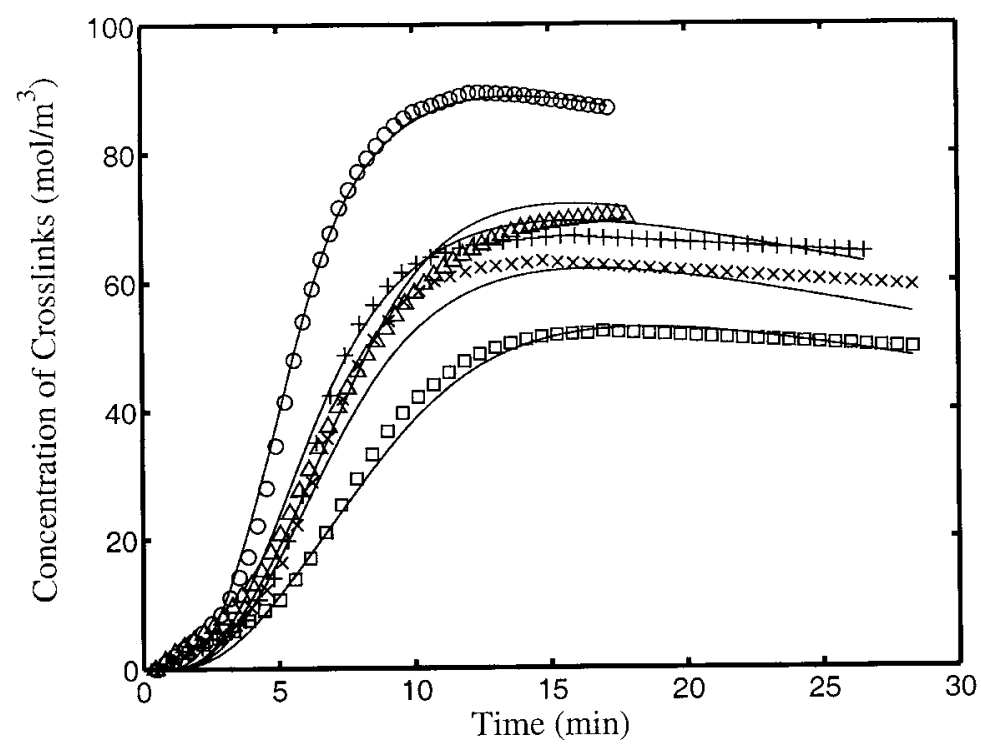

FIG. 32. - Comparison of the temporal evolution of the total (i.e. sum of all lengths) concentration of crosslinks predicted by Model-III with experimental ODR data for different formulations cured at $310^{\circ} \mathrm{F}$.

Formulations include: (o) 1 phr MBS and $4 \mathrm{phr} \mathrm{S}$; (+) 1 phr MBS and $2 \mathrm{phr} \mathrm{S}$; $(\Delta) 0.5 \mathrm{phr}$ MBS and $4 \mathrm{phr} \mathrm{S}$; (x) $0.75 \mathrm{phr}$ MBS and $2 \mathrm{phr} \mathrm{S}$; ( $\square$ ) $0.5 \mathrm{phr}$ MBS and $2 \mathrm{phr}$. The predictions are indicated by the solid lines No retarder is used and the rest of the cure-system is given in Section VI. 


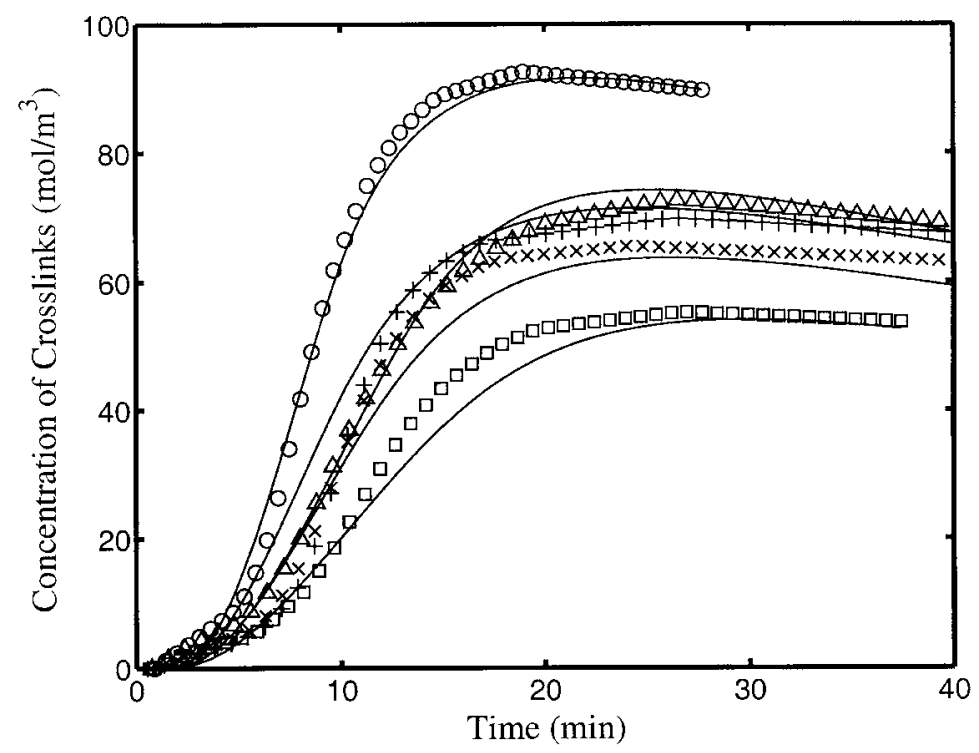

FIG. 33. - Comparison of the temporal evolution of the total (i.e. sum of all lengths) concentration of crosslinks predicted by Model-III with experimental ODR data for different formulations cured at $298^{\circ} \mathrm{F}$. Formulations include: (o) $1 \mathrm{phr}$ MBS and $4 \mathrm{phr} \mathrm{S}$; (+) $1 \mathrm{phr}$ MBS and $2 \mathrm{phr} \mathrm{S}$;

$(\Delta) 0.5 \mathrm{phr}$ MBS and $4 \mathrm{phr} \mathrm{S}$; (x) $0.75 \mathrm{phr}$ MBS and $2 \mathrm{phr} \mathrm{S}$; ( $\square$ ) $0.5 \mathrm{phr}$ MBS and $2 \mathrm{phr}$ S. The predictions are indicated by the solid lines. No retarder is used and the rest of the cure-system is given in Section 6.

Although the reduction in RMSE may be considered a criterion of model discrimination, it is not clear whether a $14 \%$ reduction in RMSE is sufficient to determine that Model-III is superior to Model-II. What is truly needed for model-discrimination is a comparison that is demonstratively superior in ODR predictive capabilities or is able to capture an important qualitative feature of one of the internal species (e.g. Model-II was able to capture the proper ordering of the $A_{x}$ species versus Model-I). The fit to the ODR data set does not, in our opinion, enable an unambiguous discrimination of Model-II versus Model-III, but the evolution and/or depletion of key intermediates like BtS-S $-\mathrm{SBt}, \mathrm{RS}-\mathrm{S}_{\mathrm{y}}-\mathrm{SBt}$, etc. might provide a way to distinguish these two models. Direct experimental data on these intermediates for the MBS-natural rubber system is not available; however, Gradwell et al. ${ }^{46}$ and Morgan et al. ${ }^{60}$ did measure the distribution of sulfurating species as a function of time on heating MBTS/S system in a $1: 1$ molar ratio at $150{ }^{\circ} \mathrm{C}$ and MBTS/S/ZnO system in a 1:1:1 molar ratio at $152{ }^{\circ} \mathrm{C}$. This data has been shown previously in Figures 5, 6 and 7, where the following trends were observed:

1. The shorter length BtS-S $-\mathrm{SBt}$ species are most abundant and are formed prior to the longer length polysulfidic accelerators.

2. The equilibrium concentrations of $B t S-S_{x}-S B t$ are very similar for the zinc-free response (see Figure 4 in Ref. 60) and the zinc-containing systems ${ }^{60}$ shown in Figure 7.

3. In the presence of $\mathrm{ZnO}$, the concentration profiles of the BtS-S $-\mathrm{SBt}$ species for $\mathrm{x} \leq 6$ exhibit a slight maximum before attaining their equilibrium concentrations as shown in Figure 7.

4. MBTS and sulfur are not fully consumed in the reaction, exhibiting a nonzero equilibrium concentration as shown in Figure 5.

The MBTS-accelerated systems with natural rubber studied by Gradwell et al. ${ }^{46}$ and Morgan $e t$ $a l .{ }^{60}$ are very similar to the MBS-accelerated system that is part of this study. Thus, the models 
should be able to qualitatively reproduce the trends shown in Figures 5 and 7, using rate constants that have already been determined for the MBS-natural rubber system. However, quantitative comparison between our PBE models and these data sets is not possible because the mass balances are not closed in the data of Gradwell et al. ${ }^{46}$ and Morgan et al. ${ }^{60}$ Specifically, since the $\mathrm{BtS}$ - unit is not changed by any of the vulcanization reactions, a mole-balance over all BtSspecies should be conserved, where BtS- is present in MBTS, MBTP, MBTM and MBT. Examining the Gradwell et al. ${ }^{46}$ data shown in Figure 5, the concentration of MBT and MBTM is almost negligible, which implies that all the BtS- species are present as MBTS and MBTP. The equilibrium concentration of MBTS is $25 \mathrm{~mol} \%$, and the equilibrium $\mathrm{mol} \%$ of the sum of MBTPs is approximately $35 \mathrm{~mol} \%$, which accounts for only $60 \%$ of the total BtS- species, leaving approximately $40 \mathrm{~mol} \%$ of BtS- unaccounted for. A similar analysis of the data for the MBTS/S/ZnO system of Morgan et al. ${ }^{60}$ also accounts for only $60 \mathrm{~mol} \%$ of the total BtSspecies.

Although a quantitative analysis of the Gradwell et al. $^{46}$ and Morgan et al. ${ }^{60}$ data is not possible, we still believe that the trends in the data are correct and may provide a way to discriminate between Model-II and Model-III. When rubber is not present and the starting material is MBTS, the defining kinetic equations for Model-II are:

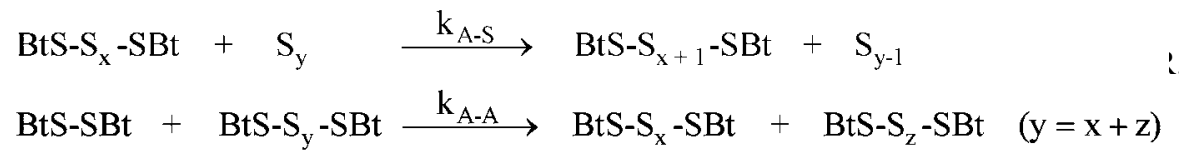

The PBEs associated with R.81 are a subset of the PBEs developed previously in Section IV. Since MBTS is a species in the MBS-natural rubber system, we will assume that the $\mathrm{k}_{\mathrm{A}-\mathrm{S}}$ and $\mathrm{k}_{\mathrm{A}-\mathrm{A}}$ rate constants that have already been determined and given in Table VIII will be reasonable for the MBTS/S system where there is no natural rubber. The predictions of Model-II, using the previously determined rate constants, are shown in Figure 34 for 1:1 molar ratio of MBTS to sulfur. Examining the predictions in Figure 34, the equilibrium concentrations of $A_{x}$ do not decrease with increasing polysulfidic length, specifically for length 6,7 and 8 , in contrast to the data shown in Figure 7. This predicted ordering of the equilibrium concentrations of $A_{x}$ with decreasing $x$ could be either the result of (i) the particular numerical values of the rate constants chosen for simulation or (ii) an incorrect reaction mechanism. In order to assess the effect of the rate constants a number of simulations with different rate constants were performed; ${ }^{138}$ however, the proper ordering of the equilibrium concentrations of the $A_{x}$ species was never achieved. Thus, it is clear from the predictions shown in Figure 34 that despite the ability to describe the ODR response, Model-II is unable to qualitatively reproduce the results in the absence of rubber and consequently needs refinement. 


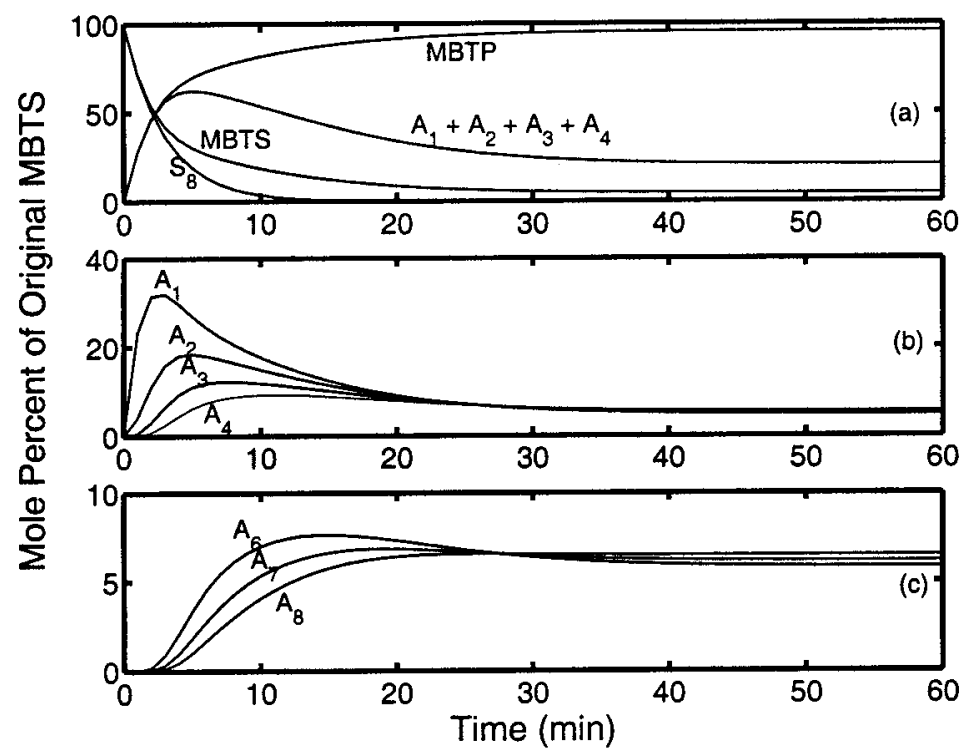

FIG. 34. - Evolution of $A_{x}$ species predicted by Model-II for the MBTS/S system in a 1:1 molar ratio at $150{ }^{\circ} \mathrm{C}$. The rate constants are based on the pre-exponential factors and activation energies regressed from ODR data for MBS-accelerated natural rubber vulcanizates using Model-II, and given in Table VIII. $\mathrm{k}_{\mathrm{A}-\mathrm{S}}=0.333 \mathrm{~m}^{3} / \mathrm{mol} \cdot \mathrm{min}$ and $\mathrm{k}_{\mathrm{A}-\mathrm{A}}=0.321 \mathrm{~m} / \mathrm{mol} \cdot \mathrm{min}$.

We will now assess if Model-III can account for the qualitative features of the experimental data. For the MBS/S system where no rubber is present, the relevant kinetic expression is given by R.80, and the associated PBEs are a subset of the Model-III equations previously described. The predictions using R.80 are shown in Figure 35, where the four rate constants $\mathrm{k}_{\mathrm{B}}, \mathrm{k}_{\mathrm{E}-\mathrm{S}}, \mathrm{k}_{\mathrm{E}-\mathrm{E}}$ and $\mathrm{k}_{\mathrm{A}-\mathrm{A}}$ are determined according to the rate constants previously developed for Model-III from the MBS-natural rubber system and as given in Table $X$. The rate constants are for the zinc-activated MBS-natural rubber system, thus the predictions can be directly compared with the experimental data shown in Figure 7, which is also for a zinc-activated system. The predictions for Model-III qualitatively agree with the experimental data, where the simulations show that (i) the shorter length $A_{x}$ are formed prior to the longer length $A_{x}$, (ii) the shorter lengths pass through a maximum before attaining their equilibrium concentrations; and (iii) the equilibrium concentrations of the various $A_{x}$ species are now properly ordered with respect to the polysulfidic length. Comparing the predictions in both Figure 34 and Figure 35 versus the trends in the experimental data, it is clear that Model-III provides a better representation of the vulcanization process. 
SULFUR VULCANIZATON OF NATURAL RUBBER FOR BENZOTHIAZOLE

TABLE IX

Reaction Scheme and the Associated Rate-Constants Considered in Model-III

\begin{tabular}{|c|c|c|c|}
\hline $\begin{array}{l}\text { Reaction } \\
\text { Number }\end{array}$ & Reaction Type & $\begin{array}{c}\text { Rate } \\
\text { Constant }\end{array}$ & $\begin{array}{c}\text { Reaction } \\
\text { Mechanism }\end{array}$ \\
\hline & Accelerator Chemistry & & \\
\hline 1 & MBS $\rightarrow$ MBT + Amine & $\mathrm{k}_{\mathrm{MBS}}$ & 7 \\
\hline 2 & $\mathrm{MBS}+\mathrm{MBT} \rightarrow \mathrm{A}_{0}+$ Amine & $\mathrm{k}_{\mathrm{MBS}}{ }^{-\mathrm{MBT}}$ & 8 \\
\hline 3 & $A_{x} \rightarrow E_{y}^{*}+E_{z}^{*}: 0 \leq x \leq 14, y+z=x$ & $\mathrm{k}_{\mathrm{B}}$ & 16,17 \\
\hline \multirow[t]{2}{*}{4} & $A_{0}+A_{x} \leftrightarrow A_{y}+A_{z}: x=y+z$ and $1 \leq x, y, z \leq 14$ & $\mathrm{k}_{\mathrm{A}-\mathrm{A}}$ & 27 \\
\hline & Crosslinking Chemistry & & \\
\hline 5 & $\mathrm{~A}_{\mathrm{x}}+$ Rubber $\rightarrow \mathrm{B}_{\mathrm{x}}+\mathrm{MBT}: 0 \leq \mathrm{x} \leq 14$ & $\mathrm{k}_{\mathrm{A}-\mathrm{R}}$ & 34 \\
\hline 6 & $\mathbf{B}_{\mathrm{x}}+$ Rubber $\rightarrow \mathbf{B}^{*_{y}}+\mathrm{E}^{*}{ }_{\mathrm{z}}: \mathbf{1} \leq \mathrm{x}, \mathrm{y}, \mathrm{z} \leq 16$ & $\mathrm{k}_{\mathrm{B}-\mathrm{R}}$ & 42 \\
\hline \multirow[t]{2}{*}{7} & $\mathrm{~B}_{\mathrm{x}}+$ Rubber $\rightarrow \mathrm{Vu}_{\mathrm{x}}: 1 \leq \mathrm{x} \leq 16$ & $\mathrm{k}_{\mathrm{VU}}$ & 43 \\
\hline & Post-Crosslinking Chemistry & & \\
\hline 8 & $\mathrm{Vu}_{\mathrm{x}} \rightarrow$ Dead Ends $: 1 \leq \mathrm{x} \leq 16$ & $\mathbf{k}_{\mathrm{DEG}}$ & 62,63 \\
\hline \multirow[t]{2}{*}{9} & $\mathrm{Vu}_{\mathrm{x}}+\mathrm{A}_{0} \rightarrow \mathrm{Vu}_{\mathrm{x}-1}+\mathrm{A}_{1}: 1 \leq \mathrm{x} \leq 16$ & $\mathrm{k}_{\text {DESULF }}$ & 60,61 \\
\hline & Other Reactions & & \\
\hline 10 & $E^{*}{ }_{x}+S_{y} \rightarrow E_{x+1}^{*}+S_{y-1}: 1 \leq x \leq 16,1 \leq y \leq 8$ & $k_{\mathrm{E}-\mathrm{S}}$ & 23 \\
\hline 11 & $\mathrm{E}^{*}{ }_{\mathrm{x}}+$ Rubber $\rightarrow \quad \mathrm{B}_{\mathrm{x}}: 0 \leq \mathrm{x} \leq 16$ & $\mathrm{k}_{\mathrm{E}-\mathrm{R}}$ & 49 \\
\hline 12 & $\mathrm{~B}^{*}{ }_{\mathrm{x}}+\mathrm{S}_{8} \rightarrow \mathrm{B}^{*}{ }_{\mathrm{x}+1}+\mathrm{S}_{\mathrm{y}-\mathrm{k}}: 1 \leq \mathrm{x} \leq 16,1 \leq \mathrm{y} \leq 8$ & $\mathrm{k}_{\mathrm{BST}-\mathrm{s}}$ & 50,51 \\
\hline 13 & $\mathrm{~B}_{\mathrm{x}}^{*}+$ Rubber $\rightarrow \mathrm{L}_{\mathrm{x}}: 1 \leq \mathrm{x} \leq 16$ & $\mathrm{k}_{\mathrm{LOOP}}$ & 52 \\
\hline 14 & $\mathrm{~B}_{\mathrm{x}}^{*}+\mathrm{A}_{0} \rightarrow \mathrm{B}_{\mathrm{x}}+\mathrm{E}_{0}: 1 \leq \mathrm{x} \leq 16$ & $\mathrm{k}_{\mathrm{A}-\mathrm{BST}}$ & 53 \\
\hline 15 & $E^{*}{ }_{2}+E^{*}{ }_{y} \rightarrow A_{x} \quad: 1 \leq x \leq 14, y+z=x$ & $k_{\mathrm{E}-\mathrm{E}}$ & 14 \\
\hline
\end{tabular}


TABLE X

Activation Energy and Pre-Exponential Factors for Model-III

\begin{tabular}{ccc}
\hline Rate-Constant & Pre-Exponential Factor & Activation Energy (kcal/mol) \\
\hline $\mathrm{k}_{\mathrm{MBS}}$ & $5.666 \times 10^{18}$ & 38.7 \\
$\mathrm{k}_{\mathrm{MBS}-\mathrm{MBT}}$ & $1.045 \times 10^{4}$ & 6.8 \\
$\mathrm{k}_{\mathrm{B}}$ & $6.125 \times 10^{25}$ & 50.5 \\
$\mathrm{k}_{\mathrm{A}-\mathrm{A}}$ & $7.157 \times 10^{1}$ & 2.6 \\
$\mathrm{k}_{\mathrm{A}-\mathrm{R}}$ & $4.755 \times 10^{6}$ & 12.9 \\
$\mathrm{k}_{\mathrm{B}-\mathrm{R}}$ & $1.432 \times 10^{6}$ & 13.3 \\
$\mathrm{k}_{\mathrm{VU}}$ & $1.654 \times 10^{4}$ & 5.9 \\
$\mathrm{k}_{\mathrm{DEG}}$ & $9.231 \times 10^{14}$ & 32.6 \\
$\mathrm{k}_{\mathrm{E}-\mathrm{S}}$ & $4.671 \times 10^{3}$ & 6.9 \\
\hline
\end{tabular}

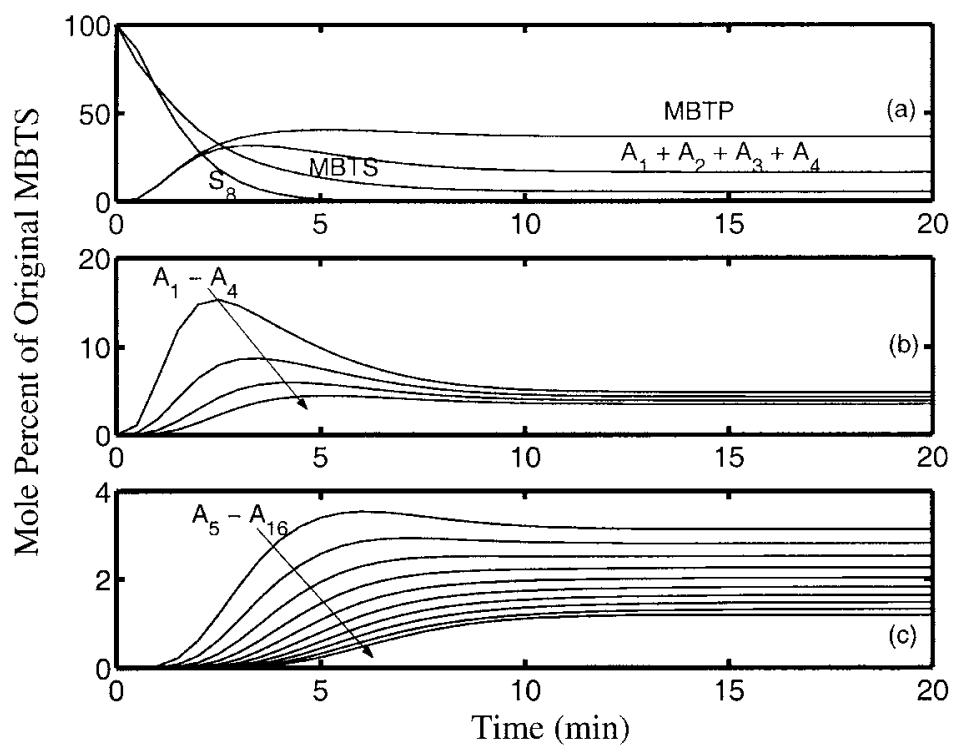

FIG. 35. - Evolution of $\mathrm{A}_{\mathrm{x}}$ species predicted by Model-III for the MBTS/S system in a 1:1 molar ratio at $150{ }^{\circ} \mathrm{C}$. The rate constants are based on the pre-exponential factors and activation energies regressed from ODR data for MBS-accelerated natural rubber vulcanizates using Model-III, and given in Table X. $\mathrm{k}_{\mathrm{E}-\mathrm{S}}=1.317 \mathrm{~m} / \mathrm{mol} \cdot \mathrm{min}$, $\mathrm{k}_{\mathrm{A}-\mathrm{A}}=3.336 \mathrm{~m}^{3} / \mathrm{mol} \cdot \mathrm{min}, \mathrm{k}_{\mathrm{E}-\mathrm{E}}=1.317 \mathrm{~m}^{3} / \mathrm{mol} \cdot \mathrm{min}$ and $\mathrm{k}_{\mathrm{B}}=0.536 \mathrm{~min}^{-1}$.

A final question concerns the role of $\mathrm{ZnO}$ in the reaction kinetics of the MBTS/S system. Specifically, why does zinc cause a more pronounced overshoot in the profiles as shown in Figure 7 versus the zinc-free system? Since MBTS does not react with $\mathrm{ZnO}$, it is not clear why $\mathrm{ZnO}$ should affect the product distribution of $\mathrm{A}_{\mathrm{x}}$ species; thus, one would anticipate that the concentration profiles for the zinc and zinc-free systems should be identical. Morgan et al. ${ }^{60}$ suggest that $\mathrm{ZnO}$ increases the rate of different reactions in the accelerator chemistry by facilitating the opening of the sulfur ring leading to faster sulfuration of $\mathrm{BtS}^{\bullet}$ radicals. We performed an exhaustive search to locate a new set of rate constants, but we were not able to find any parameter set that did not produce a maximum in the $A_{x}$ curve for small $x$. Thus, we do not currently have an explanation as to why the maximum in the $A_{x}$ curves is not seen in the zinc-free MBTS-S sys- 
tem. Irrespective of this limitation, Model-III does reconcile the accurate ordering of the equilibrium concentrations of different $\mathrm{A}_{\mathrm{x}}$. More importantly, it demonstrates how the PB-models can provide a rational framework for discriminating different reaction mechanisms, when experimental data is available on the evolution of the intermediate species.

The population balance model that has been developed is the simplest model for accelerated sulfur vulcanization consistent with the mechanistic chemistry. As shown in this Section, Model-II is able to quantitatively describe the evolution of the crosslink density for different formulations at a variety of temperatures using a single set of rate constants and associated activation energies. Model-III is also able to quantitatively describe the ODR data with a little better precision than Model-II; in addition, Model-III is able to predict the qualitative trends in the evolution of the $A_{x}$ species that were experimentally observed for related cure systems where rubber was not present.

\section{ERROR AND SENSITIVITY ANALYSIS FOR ESTIMATES OF RATE CONSTANTS}

In the previous Section, the rate constants were estimated by minimizing the sum-of-thesquare-difference between the experimental data and the model predictions. In this Section, we will critically analyze the robustness of the kinetic parameter estimates. The PBEs are a nonlinear model of the type

$$
\mathrm{y}=\mathrm{f}\left(\mathrm{x}_{\mathrm{i}}, \boldsymbol{\beta}\right)+\varepsilon_{\mathrm{i}}
$$

where $\boldsymbol{\beta}=\left\{\mathrm{k}_{\mathrm{i}}\right\}$ is the vector of parameters that are to be estimated. The variable $\mathrm{x}_{\mathrm{i}}$ defines a specific formulation, temperature and time, $f\left(x_{i}, \boldsymbol{\beta}\right)$ is the prediction of the PBEs, $y$ is the experimental data and is the error in the model. The variance of $\boldsymbol{\beta}$ is given by

$$
\operatorname{Var}(\boldsymbol{\beta})=\left(\mathrm{J}^{\mathrm{T}} \mathrm{J}\right)^{-1} \mathrm{~s}^{2} \quad \text { where } \mathrm{J}=\left.\frac{\partial \mathrm{f}(\mathrm{x}, \boldsymbol{\beta})}{\partial \boldsymbol{\beta}_{\mathrm{j}}}\right|_{\boldsymbol{\beta}=\boldsymbol{\beta}_{\mathbf{0}}}
$$

and $\boldsymbol{\beta}_{\mathbf{0}}$ is the nominal value of $\boldsymbol{\beta}$. The above equation is based on a localized linear approximation of the nonlinear function near $\boldsymbol{\beta}_{\mathbf{0}}$, assuming that the experimental error has zero mean and a constant variance $\mathrm{s}^{2}$ given by

$$
s^{2}=\frac{\sum_{i=1}^{n}\left(y_{i}-\hat{y}_{i}\right)^{2}}{n-p}
$$

where $y_{i}$ is the model predictions, $\hat{y}_{i}$ is the experimentally measured response, $n$ is the number of experimental points and $\mathrm{p}$ is the number of parameters being estimated. Using Equations 51 and 52 , the variances for the rate constant estimates and the correlation matrices for the three models have been calculated and are shown in Table XI. From the table, it may be seen that the ratio of the standard deviation to the rate constant is less than $10 \%$ for most rate constants. The accuracy with which the rate constants have been determined is excellent, especially since these rate constants were evaluated by only taking into account the crosslink density curves for different formulations. If additional experimental data for the intermediate species like $\mathrm{A}_{\mathrm{x}}$ or sulfur were available, the error in estimating the rate constants could be further minimized. 
TABLE XI

Variance and Correlation Matrices of Parameter Estimates for Models-I, II and III at $330^{\circ} \mathrm{F}$

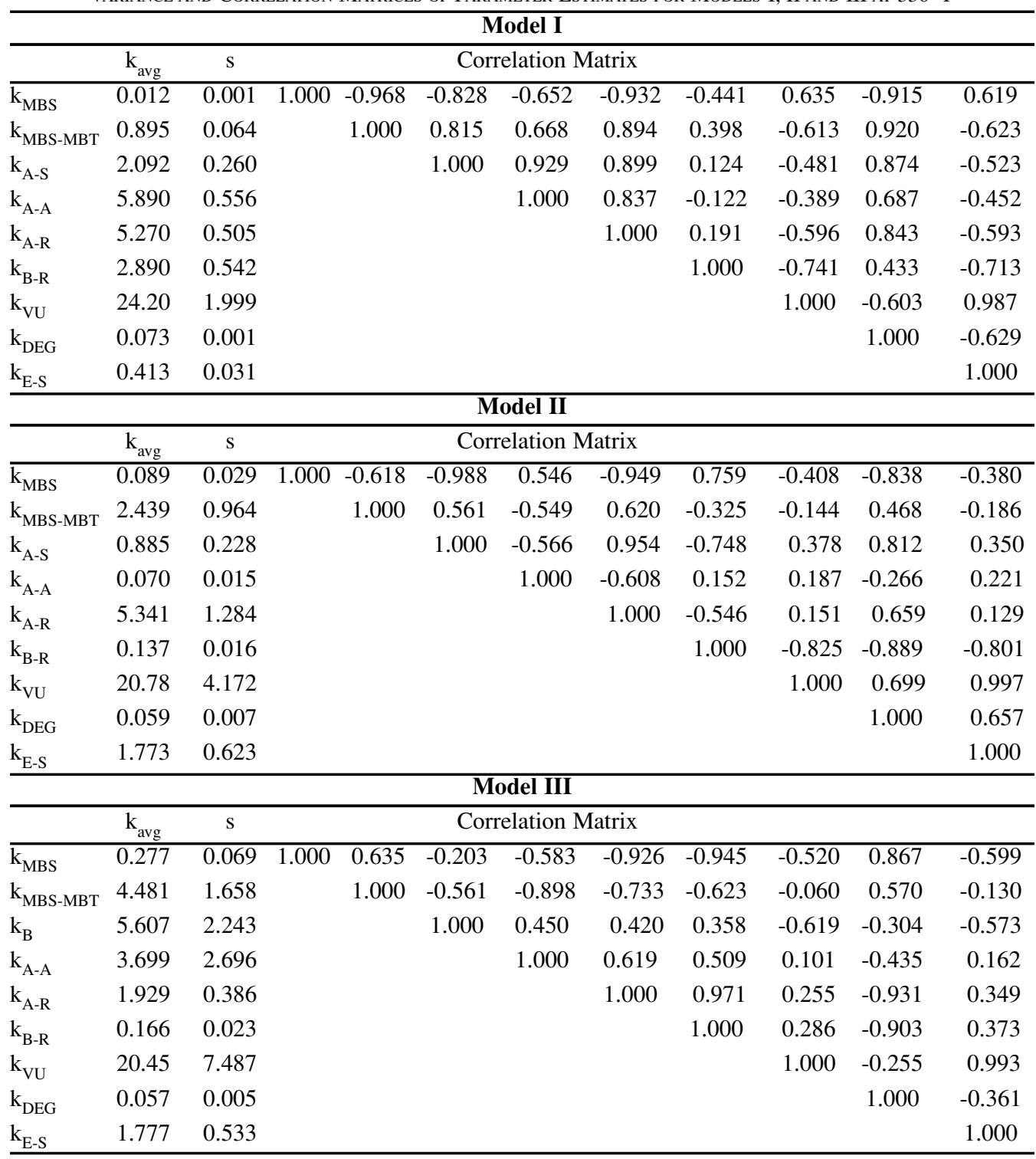

All three population balance models have nine optimizable rate constants. It is important to ascertain if these nine constant models are over-parameterized, since our objective is to develop the most parsimonious description of the vulcanization process without compromising the underlying chemistry. Principal Component Analysis (PCA) ${ }^{139}$ has been performed on the normalized sensitivity matrix $\mathrm{S}$, which is defined by 


$$
\mathbf{S}=\left[\begin{array}{cccc}
\frac{\partial \ln \left(V u_{1}^{\text {total }}\right)}{\partial \ln \left(k_{1}\right)} & \frac{\partial \ln \left(V u_{1}^{\text {total }}\right)}{\partial \ln \left(k_{2}\right)} & \cdots & \frac{\partial \ln \left(V u_{1}^{\text {total }}\right)}{\partial \ln \left(k_{p}\right)} \\
\frac{\partial \ln \left(V u_{2}^{\text {total }}\right)}{\partial \ln \left(k_{1}\right)} & \frac{\partial \ln \left(V u_{2}^{\text {total }}\right)}{\partial \ln \left(k_{2}\right)} & \cdots & \frac{\partial \ln \left(V u_{1}^{\text {otal }}\right)}{\partial \ln \left(k_{p}\right)} \\
\bullet & \\
\bullet & \frac{\partial \ln \left(V u_{q}^{\text {total }}\right)}{\partial \ln \left(k_{2}\right)} & \cdots & \frac{\partial \ln \left(V u_{q}^{\text {total }}\right)}{\partial \ln \left(k_{p}\right)}
\end{array}\right]
$$

where $k_{1}$ through $k_{p}$ are the $p$ rate constants and $t_{1}$ through $t_{q}$ are the $q$ time points across the different formulations that were optimized. The normalized sensitivity matrix has components $\partial \operatorname{lnV} u_{i}^{\text {total }} / \partial \ln k_{j}$ as opposed to the more straightforward $\partial \mathrm{V} u_{i}^{\text {total }} / \partial k_{j}$ terms. This ensures an appropriate comparison between rate constants that have different units depending upon the order (i.e. first order, second order and so on) of the reaction they control. PCA is a systematic methodology for dividing the rate constant space into influential and non-influential domains and thus provides an objective criterion for selecting a minimum set of rate constants consistent with the data. Since each rate constant is associated with a particular class of reactions, determination that a specific rate constant is irrelevant implies that the associated reaction is not necessary, at least for the data set being analyzed. PCA analysis of the sensitivity matrix provides an objective methodology for eliminating unimportant reactions.

PCA employs the response function $\mathrm{Q}(\boldsymbol{\alpha})$, which quantifies the variation on the dependent variables by variations in the set a of model parameters. Following the analysis of Vajda et al., ${ }^{139} \mathrm{Q}(\boldsymbol{\alpha})$ is defined as

$$
Q(\boldsymbol{\alpha})=\sum_{i=1}^{q}\left[\frac{V u^{\text {total }}\left(t_{i}, \boldsymbol{\alpha}\right)-V u^{\text {total }}\left(t_{i}, \boldsymbol{\alpha}^{0}\right)}{V u^{\text {total }}\left(t_{i}, \boldsymbol{\alpha}^{0}\right)}\right]^{2}
$$

where $\boldsymbol{\alpha}=\left\{\alpha_{\mathrm{i}}\right\}=\left\{1 \mathrm{n} k_{\mathrm{i}}\right\}, \mathrm{Vu}^{\text {total }}\left(\mathrm{t}_{\mathrm{i}}, \boldsymbol{\alpha}\right)$ is the output variable at time $\mathrm{t}_{\mathrm{i}}$, and $\boldsymbol{\alpha}^{\mathbf{0}}$ is the nominal value of $\boldsymbol{\alpha}$. Although $\mathrm{Q}(\boldsymbol{\alpha})$ as defined above does not explicitly incorporate the experimental data, the data is implicitly included via $\boldsymbol{\alpha}^{\mathbf{0}}$. Expanding $\mathrm{Q}(\boldsymbol{\alpha})$ about the point $\boldsymbol{\alpha}^{\mathbf{0}}$ in a Taylor series yields

$$
Q(\boldsymbol{\alpha}) \approx Q\left(\boldsymbol{\alpha}^{0}\right)+\mathbf{G}^{T}\left(\boldsymbol{\alpha}^{0}\right)\left(\boldsymbol{\alpha}-\boldsymbol{\alpha}^{0}\right)+\frac{1}{2}\left(\boldsymbol{\alpha}-\boldsymbol{\alpha}^{0}\right)^{T} \mathbf{H}\left(\boldsymbol{\alpha}^{0}\right)\left(\boldsymbol{\alpha}-\boldsymbol{\alpha}^{\mathbf{0}}\right)
$$

where $\mathbf{G}$ is the gradient vector and $\mathbf{H}$ is the Hessian matrix of Q defined by $H_{i, j}=\partial^{2} \mathrm{Q} / \partial \alpha_{i} \partial \alpha_{j}$. Since $\mathrm{Q}$ is a minimum at $\boldsymbol{\alpha}^{\mathbf{0}}, \mathrm{Q}\left(\boldsymbol{\alpha}^{\mathbf{0}}\right)=0$ and $\mathrm{G}\left(\boldsymbol{\alpha}^{\mathbf{0}}\right)=0$; thus,

$$
Q(\boldsymbol{\alpha})=\frac{1}{2}\left(\boldsymbol{\alpha}-\boldsymbol{\alpha}^{0}\right)^{T} \mathbf{H}\left(\boldsymbol{\alpha}^{0}\right)\left(\boldsymbol{\alpha}-\boldsymbol{\alpha}^{\mathbf{0}}\right)
$$


The Hessian may be expressed as $\mathbf{H}=2 \mathbf{S}^{\mathrm{T}} \mathbf{S}+\mathbf{R}$, where $\mathbf{S}$ is given by Equations 53. Using the Gauss approximation, ${ }^{140} \mathbf{R}$ can be neglected; therefore,

$$
Q(\boldsymbol{\alpha})=\left(\boldsymbol{\alpha}-\boldsymbol{\alpha}^{\mathbf{0}}\right)^{T} \mathbf{S}^{\mathbf{T}} \mathbf{S}\left(\boldsymbol{\alpha}-\boldsymbol{\alpha}^{\mathbf{0}}\right)
$$

This expression for is a quadratic form and consequently its behavior is defined by an eigenanalysis of the square matrix $\mathbf{S}^{\mathbf{T}} \mathbf{S}$; specifically,

$$
\mathbf{S}^{\mathbf{T}} \mathbf{S}=\mathbf{U} \Lambda \mathbf{U}^{\mathbf{T}}
$$

where $\mathbf{U}$ is the unitary matrix of the normalized eigenvectors and $\Lambda=\left\{\lambda_{i}\right\}$ is the diagonal matrix of eigenvalues. The eigenvalues and the normalized eigenvectors of $\mathbf{S}^{\mathbf{T}} \mathbf{S}$ for Models-I, II and III are given in Table XII along with the rate constants associated with each of the eigenvalues. The eigenvectors $u_{i}=\left(u_{i 1}, u_{i 2}, \ldots, u_{i p}\right)$ have $p$ components that are directly associated with the $p$ rate constants used in $\mathbf{S}$ as defined in Equation 53. The specific reactions associated with each of the rate constants are given in Tables V, VI and IX.

In the traditional PCA, if an eigenvalue is small compared to the maximum eigenvalue, the contribution to the response in the direction of the associated eigenvector can be neglected. However, in the current problem one cannot just arbitrarily eliminate eigenvectors, since the components of the eigenvectors are associated with reactions, i.e. what can be eliminated are reactions or sets of reactions with small eigenvalues. A particularly simple situation occurs with respect to the $\lambda_{1}$ eigenvalue for Model-II. Examining the data in Table XII, the $\lambda_{1}$ eigenvalue is eight orders of magnitude smaller than the maximum eigenvalue; in addition, the only significant component of $\mathbf{u}_{\mathbf{1}}$ is $\mathbf{u}_{1,4}$ which is associated with the polysulfidic accelerator exchange reaction. Thus, this reaction can be eliminated without any significant affect on the $\mathrm{Vu}^{\text {total }}$ response. We have eliminated this reaction in Model-II, and compared the predictions versus the experimental ODR data. The predictions without the polysulfidic exchange reaction for Model-II are identical to the results shown in Figure 21 when the exchange reactions were included. The $\lambda_{2}$ eigenvalue for Model-II is also small, being five orders of magnitude smaller than the maximum eigenvalue; however, in this case there are several nonzero components in its corresponding eigenvector. More importantly, a number of the components of $\mathbf{u}_{2}$ have significant contributions to eigenvectors with larger eigenvalues that cannot be neglected. For example, $\mathrm{u}_{2,7}=0.6216$ is the largest component of $\mathbf{u}_{2}$ and is associated with the formation of $\mathrm{Vu}_{\mathrm{x}}$ from $\mathrm{B}_{\mathrm{y}}{ }^{\bullet}$, but this reaction also has significant contributions to $\mathbf{u}_{5}, \mathbf{u}_{6}, \mathbf{u}_{7}$ and $\mathbf{u}_{\mathbf{8}}$ all of which have non-negligible eigenvalues. Consequently, the $\mathrm{Vu}_{\mathrm{x}}$ formation reaction can not be eliminated. One could potentially eliminate sets of reactions, if they are only present in a set of eigenvectors where all associated eigenvalues are negligible; however, this situation is not present for any of the eigenvectors given in Table XII. 
TABLE XII

Estimation of Eigenvalues and EigenVectors of $\mathrm{S}^{\mathrm{T}} \mathrm{S}$ in Models, II AND III Using Kinetic Parameters Optimized From ODR Data at $330^{\circ} \mathrm{F}$

\begin{tabular}{|c|c|c|c|c|c|c|c|c|c|c|}
\hline \multicolumn{11}{|c|}{ Model-I } \\
\hline Reaction & & $\lambda_{1}, u_{1}$ & $\lambda_{2}, u_{2}$ & $\lambda_{3}, u_{3}$ & $\lambda_{4}, \mathrm{u}_{4}$ & $\lambda_{5}, u_{5}$ & $\lambda_{6}, \mathrm{u}_{6}$ & $\lambda_{7}, u_{7}$ & $\lambda_{8}, u_{8}$ & $\lambda_{9}, \mathrm{u}_{9}$ \\
\hline & $\lambda_{\mathrm{i}}$ & 0.0057 & 0.0083 & 0.0192 & 0.0940 & 0.4055 & 0.7200 & 8.388 & 19.16 & 126.3 \\
\hline MBS & $u_{\mathrm{i} 1}$ & 0.0473 & -0.1414 & -0.2028 & 0.4258 & 0.4293 & -0.1209 & 0.0342 & -0.0744 & 0.7414 \\
\hline MBS-MBT & $u_{\mathrm{i} 2}$ & -0.0888 & 0.1083 & 0.2175 & -0.3147 & -0.3694 & -0.7509 & 0.0274 & -0.1190 & 0.3448 \\
\hline A-S & $u_{i 3}$ & 0.7877 & -0.0946 & -0.3799 & -0.4617 & 0.0937 & -0.0527 & 0.0156 & -0.0239 & 0.0270 \\
\hline A-A & $\mathrm{u}_{\mathrm{i} 4}$ & 0.5812 & -0.0020 & 0.7067 & 0.3868 & -0.1072 & 0.0298 & 0.0044 & -0.0285 & -0.0024 \\
\hline A-R & $u_{\mathrm{i} 5}$ & -0.0959 & 0.1840 & 0.3562 & -0.5163 & 0.0870 & 0.5267 & -0.2163 & 0.0066 & 0.4813 \\
\hline B-R & $u_{i 6}$ & -0.0488 & -0.8117 & -0.0489 & 0.0076 & -0.4916 & 0.1825 & -0.1916 & -0.0509 & 0.1487 \\
\hline $\mathrm{Vu}$ & $u_{i 7}$ & 0.0832 & 0.3533 & -0.2421 & 0.2192 & -0.2365 & 0.0504 & -0.6601 & -0.5171 & -0.0063 \\
\hline DEG & $\mathrm{u}_{\mathrm{i} 8}$ & 0.0321 & -0.0235 & 0.0138 & 0.0365 & 0.1146 & -0.2248 & -0.6317 & 0.7305 & -0.0243 \\
\hline E-S & $u_{i 9}$ & 0.1100 & 0.3759 & -0.2824 & 0.2022 & -0.5837 & 0.2326 & 0.2820 & 0.4187 & 0.2762 \\
\hline \multicolumn{11}{|c|}{ Model-II } \\
\hline \multirow[t]{2}{*}{ Reaction } & & $\lambda_{1}, \mathrm{u}_{1}$ & $\lambda_{2}, u_{2}$ & $\lambda_{3}, u_{3}$ & $\lambda_{4}, \mathrm{u}_{4}$ & $\lambda_{5}, \mathrm{u}_{5}$ & $\lambda_{6}, \mathrm{u}_{6}$ & $\lambda_{7}, \mathrm{u}_{7}$ & $\lambda_{8}, \mathrm{u}_{8}$ & $\lambda_{9}, \mathrm{u}_{9}$ \\
\hline & $\lambda_{\mathrm{i}}$ & $1.4 \times 10^{-6}$ & 0.0075 & 0.1223 & 0.2360 & 0.4377 & 0.7988 & 10.46 & 27.65 & 115.23 \\
\hline MBS & $u_{i 1}$ & 0.0001 & 0.2395 & 0.0069 & 0.4542 & 0.3641 & -0.2865 & -0.5107 & 0.1562 & 0.4862 \\
\hline MBS-MBT & $u_{i 2}$ & 0.0001 & -0.2718 & 0.9434 & -0.1167 & 0.0156 & -0.0672 & -0.0476 & 0.0146 & 0.1235 \\
\hline A-S & $u_{\mathrm{i} 3}$ & 0.0001 & -0.2114 & -0.1856 & -0.2991 & -0.6426 & -0.4346 & -0.1269 & 0.0629 & 0.4577 \\
\hline A-A & $u_{i 4}$ & 0.9999 & 0.0006 & 0.0001 & -0.0001 & -0.0002 & 0.0001 & 0.0003 & -0.0003 & 0.0001 \\
\hline$A-R$ & $u_{i 5}$ & 0.0004 & -0.3584 & -0.1889 & -0.5438 & 0.3458 & 0.3783 & -0.5164 & 0.0256 & 0.1005 \\
\hline B-R & $u_{\mathrm{i} 6}$ & -0.0002 & -0.0386 & -0.0395 & 0.0634 & 0.0897 & 0.4565 & 0.5046 & -0.0804 & 0.7180 \\
\hline $\mathrm{Vu}$ & $u_{i 7}$ & -0.0005 & 0.6216 & 0.1705 & -0.1472 & -0.3336 & 0.3242 & -0.3343 & -0.4790 & 0.0727 \\
\hline DEG & $u_{i 8}$ & 0.0002 & -0.0007 & 0.0624 & 0.2607 & -0.4145 & 0.4918 & -0.1933 & 0.6874 & -0.0677 \\
\hline E-S & $\mathrm{u}_{\mathrm{i} 9}$ & -0.0002 & 0.5548 & 0.0727 & -0.5489 & 0.2085 & -0.1613 & 0.2245 & 0.5122 & 0.0584 \\
\hline \multicolumn{11}{|c|}{ Model-III } \\
\hline \multirow[t]{2}{*}{ Reaction } & & $\lambda_{1}, \mathrm{u}_{1}$ & $\lambda_{2}, \mathrm{u}_{2}$ & $\lambda_{3}, u_{3}$ & $\lambda_{4}, \mathrm{u}_{4}$ & $\lambda_{5}, \mathrm{u}_{5}$ & $\lambda_{6}, \mathrm{u}_{6}$ & $\lambda_{7}, u_{7}$ & $\lambda_{8}, \mathrm{u}_{8}$ & $\lambda_{9}, \mathrm{u}_{9}$ \\
\hline & $\lambda_{\mathrm{i}}$ & 0.0026 & 0.0044 & 0.0142 & 0.0938 & 0.7714 & 1.048 & 7.782 & 33.29 & 139.5 \\
\hline MBS & $u_{i 1}$ & -0.0865 & 0.0335 & 0.0192 & -0.0720 & -0.8157 & 0.0193 & 0.2334 & -0.2127 & 0.4694 \\
\hline MBS-MBT & $u_{\mathrm{i} 2}$ & -0.1927 & -0.5517 & 0.7918 & -0.1437 & 0.0643 & -0.0231 & 0.0759 & -0.0117 & 0.0191 \\
\hline B & $u_{i 3}$ & 0.1548 & 0.0667 & 0.1242 & 0.6902 & 0.2072 & -0.2472 & 0.5834 & -0.0997 & 0.1595 \\
\hline A-A & $u_{i 4}$ & 0.6449 & 0.5296 & 0.5074 & -0.1787 & -0.0388 & 0.0324 & -0.1063 & -0.0192 & 0.0082 \\
\hline A-R & $u_{i 5}$ & 0.7159 & -0.6273 & -0.2693 & -0.0577 & -0.1073 & 0.0160 & 0.0653 & 0.0404 & -0.0224 \\
\hline B-R & $u_{i 6}$ & 0.0434 & -0.0674 & -0.0382 & 0.0696 & 0.3865 & 0.4871 & -0.2457 & -0.3961 & 0.6191 \\
\hline $\mathrm{Vu}$ & $u_{i 7}$ & -0.0232 & 0.0757 & -0.1064 & -0.4783 & 0.1837 & 0.1814 & 0.5903 & -0.5049 & -0.2891 \\
\hline DEG & $\mathrm{u}_{\mathrm{i} 8}$ & -0.0231 & 0.0550 & 0.0350 & 0.0307 & -0.0283 & 0.6917 & 0.3599 & 0.6206 & 0.0197 \\
\hline E-S & $u_{\mathrm{i} 9}$ & -0.0051 & 0.0530 & -0.1158 & -0.4775 & 0.3010 & -0.4337 & 0.2132 & 0.3817 & 0.5347 \\
\hline
\end{tabular}


The PCA developed in this Section provides a rational method for determining if a reaction can be eliminated. The data in Table XII for Model-I clearly indicates that all nine reactions are required to fit the ODR data. Likewise, the eigen-data in Table XII also indicates that all nine reactions are required in Model-III to fit the ODR data. In contrast, the eigen-data for Model-II clearly indicates that the accelerator exchange reactions given by R.27 are not needed to describe the ODR data. Although the PCA suggests that we can eliminate the accelerator exchange reaction, it must be remembered that the PCA analysis is purely mathematical. From a chemical consideration, $\mathrm{k}_{\mathrm{A}-\mathrm{A}}$ controls the exchange of sulfur between accelerator polysulfides, which may be an important reaction in the accelerator chemistry for formulations with high accelerator/sulfur ratios. Since the rate constants in this model were determined for formulations with an accelerator to sulfur ratio of less than one, the accelerator exchange reaction may not be significant for these data. If the ODR data included a larger range of formulations, the accelerator exchange reactions might very well become significant in a subsequent PCA analysis. In addition, if some of the individual $A_{x}$ species were measured, the accelerator exchange reactions might be required to accurately describe this additional data. With the exception of the accelerator exchange reactions for Model-II, PCA establishes conclusively that the PBE models considered in this paper are a parsimonious description of the vulcanization kinetics. There is no simpler description of the reaction kinetics for MBS accelerated sulfur vulcanization consistent with both the mechanistic chemistry as presented in Section III and the ODR data.

\section{DISCUSSION}

The objective of this paper is to begin the development of a quantitative, fundamental kinetic model for accelerated sulfur vulcanization. The key requirements of this approach include: (i) the relevant chemical reaction mechanisms are employed; (ii) the polysulfidic nature of the various molecular species are explicitly incorporated, and (iii) the structure of the model is critically analyzed to ensure that the kinetic model is the most parsimonious description of vulcanization process consistent with the chemical reaction mechanisms. The results presented in Section VII clearly show the efficacy of this approach. We have employed this methodology for three different models, which had different assumptions concerning the mechanism of how the accelerator and sulfur react. Because the approach naturally describes how the different length polysulfidic species evolve, we were able to use qualitative data on the relative temporal evolution of the various polysulfides from different, but related, vulcanization systems to clearly determine that only one of the proposed vulcanization models is viable.

A comprehensive, critical review of the proposed mechanisms for sulfenamide accelerated vulcanization was presented in Section III, where the requirement of chemical consistency was imposed. Specifically, the majority of the vulcanization reactions involved the breaking of a polysulfidic chain, the reaction of a persulfenyl species, or a rearrangement between two polysulfidic chains. Although the organic end groups may differ from one reaction to another, it is reasonable to assume that for structurally similar compounds, the basic reactions are affected in only a quantitative, but not a qualitative, manner. For example, the two radicals $\mathrm{R}_{-} \mathrm{SS}_{\mathrm{x}}{ }^{*}$ and $\mathrm{BtSS}_{\mathrm{x}}{ }^{*}$ only differ in the fact that end group on $\mathrm{R}_{-} \mathrm{SS}_{\mathrm{x}}{ }^{*}$ is polyisoprene, while the end group on $\mathrm{BtSS}_{\mathrm{x}}{ }^{*}$ is a benzothiazole. Consequently, if $\mathrm{BtSS}_{\mathrm{x}}{ }^{*}$ is assumed to pick-up sulfur, then $\mathrm{R}-\mathrm{SS}_{\mathrm{x}}{ }^{*}$ should also participate in a similar reaction. The requirement of chemical consistency leads to a more detailed reaction network for vulcanization; specifically, the reaction network that has been proposed in this paper as shown in Figure 8 is chemically consistent and thorough, in contrast to the traditional reaction scheme of $\mathrm{A} \rightarrow \mathrm{B} \rightarrow \mathrm{B}^{*} \rightarrow \mathrm{Vu}$ shown in Figure 1. Although the $\mathrm{A} \rightarrow \mathrm{B} \rightarrow \mathrm{B}^{*} \rightarrow \mathrm{Vu}$ reaction scheme may appear to be simpler, it is actually more complex, since it implicitly assumes that chemically similar species react in fundamentally different ways. This requirement of chemical consistency is not just an academic exercise; rather, there are important 
consequences. For example, consider how the concentration of the accelerator affects the crosslink density just prior to reversion. The experimental data shown in Figure 13 show that as the concentration of the MBS accelerator is decreased by $50 \%$ from $1.0 \mathrm{phr}$ to $0.5 \mathrm{phr}$, keeping the concentration of all other constituents constant, the maximum crosslink density decreases by $70 \%$. The vulcanization mechanism shown in Figure 8 predict a $70 \%$ reduction in agreement with the data, while the series of first order reactions associated with the $A \rightarrow B \rightarrow B^{*} \rightarrow$ Vu mechanism predict only a $50 \%$ reduction. As discussed previously with respect to R.44, an important co-product of the $\mathrm{B} \rightarrow \mathrm{B}^{*}$ reaction is $\mathrm{E}^{*}$, where chemical consistency demands that $\mathrm{E}^{*}$ regenerates A via R.16, regenerates B via R.49 and reacts with sulfur to form longer length radicals via R.23. Consequently the reaction scheme is more complex than $A \rightarrow B \rightarrow B^{*} \rightarrow V u$ as shown below:

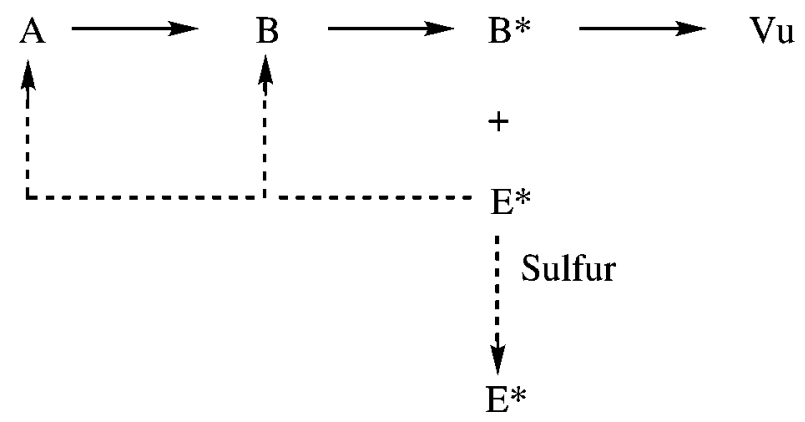

where the dotted lines indicate the new feedback mechanism due to $\mathrm{E}^{*}$. Because of this feedback mechanism, $\mathrm{E}^{*}$ enables each polysulfidic accelerator $\mathrm{A}_{\mathrm{x}}$ to generate more than one crosslink. The feedback loop shown in R.82 along with the explicit accounting for the polysulfidic nature of the accelerator species is what enables the kinetic model developed in this paper to capture how the maximum crosslink density in the ODR data depends upon the concentration of MBS. There are, however, situations where species that may appear to be chemically similar react in qualitatively different ways. For example, all $\mathrm{B}_{\mathrm{x}}$ species appear similar and thus should have similar reactivities; however, $\mathrm{B}_{\mathrm{x}}$ forms $\mathrm{B}_{\mathrm{x}}{ }^{*}$ for all polysulfidic lengths except when $\mathrm{x}$ is equal to 0 , i.e. $\mathrm{B}_{\mathrm{o}}$ is a dead end. Nevertheless, chemical consistency would again demand that if R-SBt (i.e. $\mathrm{B}_{\mathrm{o}}$ ) does not undergo additional reactions then Bt-S-Bt should also be unreactive.

In addition to chemical consistency, we have required that the mass balance be closed for all reactions, which requires that the polysulfidic nature of every species be explicitly recognized. We have employed population balances to explicitly account for the different polysulfidic lengths for all of the species that participate in the vulcanization reactions. Lumped parameter models (see Section II) suppress all polysulfidic length information; consequently, it is not possible to close a sulfur mass balance around the individual reactions - a very serious shortcoming in any fundamental kinetic model. In addition, a number of important reactions in vulcanization chemistry involve rearrangement between different polysulfidic species; for example, consider the rearrangement between sulfurating species in the accelerator chemistry (i.e. R.27) or desulfuration reactions in the post-crosslinking chemistry (i.e. R.60 and R.61). It is not possible to model these rearrangement reactions if length information is not explicitly included in the balance equations. The importance of the PBE approach is clearly illustrated by the need to predict the length distribution of the crosslinks. For example, the Formulations B and C in Figure 26 have nearly the same total number of crosslinks but widely different length distribution. Specifically, Formulation $\mathrm{C}$ that has a higher accelerator to sulfur ratio contains a higher percentage of monosulfidic and disulfidic crosslinks as compared to Formulation B that has a higher percentage of polysulfidic crosslinks. Since the total concentration of crosslinks of different lengths is equiva- 
lent in both these formulations at the end of cure, their strengths at the end of cure are equivalent. However, at longer times, the higher percentage of polysulfidic crosslinks in Formulation B would make it more susceptible to reversion as compared to Formulation C. Therefore, from the point of minimizing reversion and thus having higher strength for a longer time, Formulation $\mathrm{C}$ is better.

The vulcanization chemistry as reviewed in Section III is complex, and although there is agreement on the reaction mechanisms for much of chemistry, there are some differences on what are correct reaction mechanisms. The PBEs require the selection of particular set of reactions schemes; however, it is relatively easy to change a particular step in the reaction and determine if the new reaction scheme is better. Based on the simulations reported in this paper in conjunction with the substantial literature on sulfur vulcanization with sulfenamide accelerators reviewed in Section III, we believe the following is the most appropriate description of the vulcanization process. The chemistry of MBS accelerated vulcanization initiates with the formation of a key sulfurating intermediate MBTS via R.7 and R.8, where both polar (i.e. R.9) and radical (i.e. R.11) reaction intermediates have been proposed, although the PBEs are not affected by this detail. Once MBTS is formed, it reacts with sulfur to form the accelerator polysulfides, where the sulfur insertion reactions proceed via sequential sulfur insertion as was clearly shown in Section VII.B. Furthermore, the predictions of Model-III indicate that the sulfur insertion reactions most probably proceed by the initial splitting of the accelerator polysulfides species into $\mathrm{BtS}-\mathrm{S}_{\mathrm{x}} \cdot$ radicals, which subsequently react with sulfur followed by recombination to yield longer length polysulfides. The accelerator polysulfides react with rubber to yield crosslink precursors, where the precursor formation reaction most likely proceeds by a concerted mechanism via R.34 in the absence of zinc or by a polar mechanism via R.37 in the presence of zinc (see Section III.B.1). With respect to the PBEs, the difference between R.34 and R.37 is unimportant, since both reactions are of the form $\mathrm{A}_{\mathrm{x}} \rightarrow \mathrm{B}_{\mathrm{x}}+\mathrm{MBT}$ and thus have the same kinetic expression. Two mechanisms have been proposed for how crosslink precursors form crosslinks: (i) by the direct reaction with another rubber molecule, proceeding through a persulfenyl intermediate (i.e. R.42 and R.43) or (ii) by the combination of two precursor molecules (i.e. R.38 and R.39). The PBEs developed in this paper employ the first mechanism, since we believe that diffusion of two bulky molecules as required in the second mechanism are unlikely (see Section III.B.2). The crosslinks continue to react by a combination of desulfuration and degradation reactions that have been incorporated in the PBEs based on R.60, R.61 and R.52 respectively. Two reaction mechanisms have been proposed for how CTP retards the vulcanization process (see Section III.D). First, the CTP retarder is assumed to react with MBT (i.e. R.68) and, thus, delay the formation of MBTS and the polysulfidic accelerators; ${ }^{40,121}$ alternatively, CTP is assumed not to delay the formation of the crosslinks but only to inhibit the rate of crosslink formation. ${ }^{33,120}$ The proposed reaction of the polysulfidic crosslink precursors with CTP requires a trimolecular reaction (i.e. Step 3 in R.73) which seems rather unlikely for bulky molecules. Thus, we have not employed the retardation mechanism outlined in R.73 in the PBEs. Consequently, the PBEs have employed R.68, where the reaction of CTP with MBT effectively stops all reactions that are downstream from MBT; however, the reaction of CDB with MBT (i.e. R.70), which is often overlooked, is equally important. Unless the accelerator portion of the retarder is recycled back into the vulcanization process, not enough accelerator will be available, which would have a significant consequence on the sulfur consumption. In our opinion, the reaction mechanism described in this paragraph and summarized in Figure 8 is the most reasonable description of benzothiazole accelerated sulfur vulcanization of natural rubber.

One area in the proposed vulcanization model that needs improvement is a more detailed understanding of the role of the zinc activator. In the PBEs we have attempted to explicitly acknowledge each of the participating species. However, a certain level of lumped representation 
could not be avoided; specifically, no distinction was made between zinc-free species like BtS$\mathrm{S}_{\mathrm{x}}-\mathrm{SBt}, \mathrm{R}-\mathrm{S}_{\mathrm{x}}-\mathrm{SBt}$, etc. and the analogous zinc-complexed species. This lumping is not an inherent limitation of the PBE modeling framework, since it is straightforward to include both zincfree and zinc-complexed species with different rate constants for each species. However, we did not have ODR data where the amount of zinc oxide was systematically varied; consequently, there was no possibility to effectively regress the rate constants. When the necessary ODR data becomes available, the effects of zinc-free and zinc-complexed structures on the vulcanization chemistry can be addressed. In addition, it is important to more precisely determine how amine ligands that are associated with the zinc complex participate in the vulcanization process. Here again the population balance models can provide valuable information, when ODR data for these systems is available. Finally, the current PBE model does not consider the formation of $\mathrm{ZnS}$, although it is known that $\mathrm{ZnS}$ is formed during vulcanization reactions. $\mathrm{ZnS}$ has not been included in the current model, because the mechanism for formation of $\mathrm{ZnS}$ is not known. Possible mechanisms for the generation of $\mathrm{ZnS}$ include (i) the formation of crosslink precursors from the zinc-complexed sulfurating species (i.e. R.37), the conversion of zinc-complexed crosslink precursors to crosslinks (R.41) and the reaction of $\mathrm{H}_{2} \mathrm{~S}$ with $\mathrm{ZnO}$. Here again, when a more detailed description of the chemical mechanism for the formation of $\mathrm{ZnS}$ becomes available, it can be readily accommodated in the PBEs.

The vulcanization reaction scheme summarized in Figure 8 was deduced by a combination of the mechanistic chemistry reviewed in Section III, quantitative fitting of the PBEs to the experimental ODR data, and the qualitative comparison of the predictions of the PBEs with evolution of intermediates for related systems. The information that is learned from how the intermediates evolve, even if it is only qualitative, is very important, e.g. the relative evolution of the various BtS-S $\mathrm{x}_{\mathrm{x}}-\mathrm{SBt}$ species was used to show that only Model-III was consistent with all the experiments (see Sections VII.A and VII.B). Additional information on the behavior of intermediate species would be valuable. For example, as discussed in Section III.B.2 it has been postulated that crosslinks may be formed (i) directly from the crosslink precursors from a persulfenyl intermediate reacting with the allylic carbon in the rubber via R.42 and R.43 or (ii) by the recombination of two crosslink precursors via R.38 and R.39. From diffusional considerations we have argued that R.42 and R.43 is the correct reaction pathway and this is the mechanism that has been employed in the PBEs, although direct experimental evidence is not available. If the crosslinks are formed directly from the precursor via R.42 and R.43, the depletion of the precursor would be governed by first order kinetics versus second order kinetics if the reaction is between two crosslink precursors. Thus, if experimental data, even qualitative data, on the evolution of crosslink precursors were available, the comparison of this data with the predictions of the PBEs could definitively determine which is the actual mechanism of crosslink formation.

The PBEs require the specification of the rate constants for each the reactions. The most general formulation of the PBEs would result in a large number of rate constants, where each individual bond in the polysulfidic chain would have a different reactivity. We have significantly reduced the number of rate constants by making the reasonable, although not completely accurate, assumption that the dissociation energies, and hence the reactivities, for all the bonds in a polysulfidic chain are identical. Alternatively, one could assume that a particular bond in the polysulfidic chain (e.g. a bond that can complex with a zinc activator) has a significantly different reactivity. Using the heuristic of equal reactivity for the various bonds in the polysulfidic chain, a total of fifteen rate constants were employed, corresponding to the fifteen different reaction classes as have been identified in Tables V, VI and XIV for the three different models considered in Section VII. Four of the rate constants $\mathrm{k}_{\mathrm{A}-\mathrm{R}}, \mathrm{k}_{\mathrm{B}-\mathrm{R}}, \mathrm{k}_{\mathrm{VU}}$ and $\mathrm{k}_{\mathrm{A}-\mathrm{BST}}$ are identical to the four rate constants used previously by Coran and coworkers ${ }^{19,-23}$ to describe the induction and curing part of the vulcanization process. The rate constants $\mathrm{k}_{\mathrm{MBS}}, \mathrm{k}_{\mathrm{MBS}-\mathrm{MBT}}, \mathrm{k}_{\mathrm{A}-\mathrm{S}}$ and $\mathrm{k}_{\mathrm{A}-\mathrm{A}}$ are 
necessary to specify the kinetics of formation of the sulfurating species from the accelerator and sulfur. If MBTS is used as the accelerator instead of MBS, the rate constants $\mathrm{k}_{\mathrm{MBS}}$ and $\mathrm{k}_{\mathrm{MBS}-\mathrm{MBT}}$ are not needed. The lumped parameter model discussed in Section II, did not need to specify these rate constants, but instead required knowledge of the initial concentration of BtS-S $-\mathrm{SBt}$ or $\mathrm{BtS}-\mathrm{S}_{\mathrm{x}}-\mathrm{Zn}-\mathrm{SBt}$, which is difficult to measure and obviously not predictive. The rate constants $\mathrm{k}_{\mathrm{DEG}}$ and $\mathrm{k}_{\mathrm{DESULF}}$ are necessary to describe the degradation and desulfuration reactions during the postcrosslinking chemistry. Finally, the rate constants $\mathrm{k}_{\mathrm{E}-\mathrm{E}}, \mathrm{k}_{\mathrm{E}-\mathrm{R}}, \mathrm{k}_{\mathrm{E}-\mathrm{S}}, \mathrm{k}_{\mathrm{BST}-\mathrm{S}}$ and $\mathrm{k}_{\mathrm{LOOP}}$ are needed to describe (i) regeneration of the sulfurating species by $\mathrm{BtSS}_{\mathrm{x}}{ }^{\circ}$ recombination, (ii) formation of crosslink precursors from $\mathrm{BtSS}_{\mathrm{x}}{ }^{\cdot}$, (iii) sulfur activation by $\mathrm{BtSS}_{\mathrm{x}}{ }^{\circ}$, (iv) sulfur activation by persulfenyl radicals $\operatorname{RSS}_{\mathrm{y}}{ }^{\bullet}$, and (v) conversion of $\operatorname{RSS}_{\mathrm{y}}{ }^{\bullet}$ to cyclic sulfides. However, all these fifteen rate constants are not independent. Further reduction to the final set of nine rate constants is achieved by (i) conformational analysis to relate $\mathrm{k}_{\mathrm{LOOP}}$ to $\mathrm{k}_{\mathrm{Vu}}$ and (ii) exploiting the similarity in the chemistry between some of the reaction classes. Specifically, exchange reactions between accelerator polysulfides and desulfuration reactions between the crosslinks and the accelerator polysulfide in postcrosslinking chemistry are chemically similar and therefore we have employed the same rate constant. Likewise, the reactions of persulfenyl (i.e. R-S $\mathrm{S}_{\mathrm{x}}{ }^{\text {) }}$ ) and benzothiazole terminated polysulfidic radicals (i.e. $\mathrm{BtS}_{\mathrm{x}}{ }^{\circ}$ ) with sulfur are similar, thus, the rate constants are assumed to be identical. The reactions of these radicals with an allylic hydrogen to form crosslinks or crosslink precursors are similar and these rate constants are also assumed to be identical. Using these simplifications, the final set of nine rate constants are $\mathrm{k}_{\mathrm{MBS}}, \mathrm{k}_{\mathrm{MBS}-\mathrm{MBT}}$, $\mathrm{k}_{\mathrm{A}-\mathrm{S}}, \mathrm{k}_{\mathrm{A}-\mathrm{A}}, \mathrm{k}_{\mathrm{A}-\mathrm{R}}, \mathrm{k}_{\mathrm{B}-\mathrm{R}}, \mathrm{k}_{\mathrm{VU}}, \mathrm{k}_{\mathrm{DEG}}$ and $\mathrm{k}_{\mathrm{E}-\mathrm{S}}$ in Models-I and II, and in Model-III $\mathrm{k}_{\mathrm{A}-\mathrm{S}}$ is replaced with $\mathrm{k}_{\mathrm{B}}$. Although it would most certainly be desirable to have fewer rate constants, we shown in Section VII.C that each of the reactions and their associated rate constants that have been employed in the proposed PBE models are essential and no further reduction in rate constants is possible.

A potential advantage of the population balance approach is that a direct connection with the predictions can be now made to computational chemistry methods that are currently available. Using modern $a b$ initio and semi-empirical quantum chemistry methods, ${ }^{141}$ one can accurately estimate bond strengths, conformation energies, etc. and even the energetics of the transition states. The population balance model naturally incorporates this type of detailed information; in contrast, the kinetic constants in a lumped parameter model are implicitly averaged over the population of individual bonds, conformations, etc., which makes it impossible to have a direct connection with computational chemistry. Because of the complexity of the vulcanization process, the application of modern computational chemistry will not be easy and will never replace the need for determining at least some of the rate constants from experimental data. However, the population balance methods developed in this paper will at least provide an opportunity to begin to more effectively use the currently available computational chemistry methods to model the vulcanization process.

The focus of this paper has been the kinetics of sulfur vulcanization of natural rubber with sulfenamide accelerators, in particular MBS. There are obvious similarities between the chemistry of MBS-accelerated system and other members of the benzothiazolesulfenamide class like TBBS, CBS, DCBS, etc. And other classes of accelerators like thiurams (i.e. TMTM and TMTD) and dithiocarbamates (i.e. ZDMC and ZDEC) also involve the formation of polysulfidic species with various organic end groups. Although there will most certainly be differences in the reaction mechanisms for these different types of accelerators, the population balance framework can readily accommodate these different accelerator systems. The framework can also be used to describe mixed accelerator systems like thiuram-sulfenamides, where one would have to keep track of considerably more species, since the organic end group of the various polysulfidic intermediate can be two sulfenamides, two thiurams, or one sulfenamide and one thiuram. However, 
we anticipate that the set of rate constants in the mixed accelerator system, which is what is difficult to determine, would most probably be the same as that which has been determined for the single accelerator systems. In addition to being able to address other accelerator systems, the PBEs developed in this paper can be easily extended to sulfur vulcanization of other elastomers. For elastomers like SBR, where there is an abundance of allylic carbons, the same PBEs developed in this paper for natural rubber can be used, although the rate constants will most probably be different. For systems like butyl or nitrile rubber, where there are only a limited number of allylic carbons, the PBEs will have to be substantially modified; however, the basic approach will still be valid. We believe that the framework developed in this paper for MBS accelerated sulfur vulcanization of natural rubber has the potential to be used for different and more complex accelerator systems and a variety of elastomers.

The PBE-models developed in this paper are certainly more complex than any existing kinetic model for vulcanization in the literature. Nevertheless, they pose little difficulty for even a modest PC or workstation. For a given composition, solution of the population balance equations for 50 time-points takes less than 10 seconds on a $400 \mathrm{MHz}$ Pentium processor. The increased computational complexity is certainly a minor price to pay for the improvement in predictive capability as well as the ability to employ a more complete description of the underlying molecular reaction mechanisms.

The principal contribution of the work reported in this paper has been to unify the complex reaction network of vulcanization chemistry via a rational mathematical description using population balance methods. The predictions of the population balance models clearly indicate the success of this approach in describing the induction, curing and reversion phases of the vulcanization process for various sulfur, accelerator and retarder concentrations cured. To the best of our knowledge, this is the first kinetic model that describes the entire cure curve, explicitly acknowledges the underlying molecular mechanisms and only uses a single set of rate constants for a wide range of compositions. We hope that the results of this paper provide at least a modest advance in the understanding and quantitative kinetic modeling of accelerated vulcanization of elastomers.

\section{ACKNOWLEDGEMENTS}

This work has been supported by a grant from Caterpillar, Inc. and by the $21^{\text {st }}$ Century Research and Technology Fund. SK and PP were recipients of Purdue Research Foundation fellowships.

\section{REFERENCES}

${ }^{1}$ W. Scheele, O. Lorenz, and W. Dummer, Rubber Chem. Technol. 29, 1 (1956).

${ }^{2}$ L, Bateman, C.G. Moore, M. Porter, and B. Saville in "The Chemistry and Physics of Rubber like Substances," L

Bateman, Ed., Maclaren \& Sons Ltd., London, Wiley and Sons, 1963, pp 449.

${ }^{3}$ P. J.Nieuwenhuizen, J. Reedijk, M. van Duin, and W.J. McGill, Rubber Chem. Technol. 70, 368 (1997).

${ }^{4}$ M Porter in “The Chemistry of Sulfides," A. V. Tobolsky, Ed., John Wiley \& Sons Inc., New York (1968), pp 165.

${ }^{5}$ M. Porter in "Organic Chemistry of Sulfur," S. Oae, Ed., Plenum Press, N.Y., 1977, pp 71.

${ }^{6}$ W. Scheele, Rubber Chem. Technol. 34, 1306 (1961).

${ }^{7}$ B. Saville and A. A. Watson, Rubber Chem. TeChnol. 40, 100 (1967).

${ }^{8}$ C. D. Trivette, E. Morita, and O.W. Maender, Rubber Chem. Technol. 50, 570 (1973).

${ }^{9}$ E. Morita, Rubber Chem. Technol. 393 (1983).

${ }^{10}$ J. A. McCleverty, N. Spencer, N.A. Bailey, and S.L. Shackleton, J. Chem. Soc. Dalton Trans. 10, 1939 (1980). 
${ }^{11}$ J. A. McCleverty, N. J. Morrison, N. Spencer, C.C. Ashworth, N.A. Bailey, M.R. Johnson, J.M.A. Smith, B.A. Tabbinner, and C.R. Taylor, J. Chem. Soc. Dalton Trans. 10, 1945 (1980).

${ }^{12}$ J. A. McCleverty, R. S. Z. Kowalski, N. A. Bailey, H. Adams, K. Lumbard, and M.A. Murphy, J. Chem. Soc. Dalton Trans. 3, 493 (1982).

${ }^{13}$ J. A. McCleverty, and N. Spencer, Transit. Metal. Chem. 9(4), 139 (1984).

${ }^{14}$ J. A.McCleverty, and N. Spencer, Transit. Metal Chem. 12(1), 1 (1987).

${ }^{15}$ M. R. Kresja and J. L. Koenig, Rubber Chem. TeChnol. 66, 376 (1993).

${ }^{16}$ N. J. Morrison and M. Porter, Rubber Chem. Technol. 57, 63 (1984).

${ }^{17}$ B. Jurkowska and B. Jurkoswski, Kautsch. Gummi Kunstst. 51, 534 (1998).

${ }^{18}$ G. K. Taylor, E. S. Castner, and V. Gallatsatos, J. Chem. Soc. Faraday Trans. 91, 2655 (1995).

${ }^{19}$ A. Y. Coran, Rubber Chem. Technol. 37, 673 (1964).

${ }^{20}$ A. Y. Coran, Rubber Chem. TeChNol. 37, 679 (1964).

${ }^{21}$ A. Y. Coran, Rubber Chem. TeChnol. 37, 689 (1964).

${ }^{22}$ R. H. Campbell and R. W. Wise, Rubber Chem. Technol. 37, 635 (1964).

${ }^{23}$ R. H. Campbell and R. W. Wise, Rubber Chem. Technol. 37, 650 (1964).

${ }^{24}$ R. Ding, A. I. Leonov, and A.Y. Coran, Rubber Chem. Technol. 69, 81 (1996).

${ }^{25}$ R. Ding and A. I. Leonov, J. Appl. Polym. Sci. 61, 455 (1996).

${ }^{26}$ D. Chapman, J. Elast. Plastics 10, 129 (1978).

${ }^{27}$ V. Duchacek, J. Appl. Poly. Sci. 15, 2079 (1971).

${ }^{28}$ V. Duchacek, J. Appl. Poly. Sci. 18, 125 (1974).

${ }^{29}$ V. Duchacek, J. Appl. Poly. Sci. 19, 1617 (1975).

${ }^{30}$ A. B. Sullivan, C. J. Hann, G. H. Kuhls, Rubber Chem. Technol. 65, 488 (1992).

${ }^{31}$ M. R. Kresja and J. L. Koenig, Rubber Chem. Technol. 66, 376 (1993).

${ }^{32}$ M.H.S. Gradwell and W.J. McGill, J. Appl. Polym. Sci. 58, 2193 (1995).

${ }^{33}$ M.H.S. Gradwell, K. G. Hendrikse, and W.J. McGill, J. Appl. Polym. Sci. 66, 1093 (1997).

${ }^{34}$ M.R. Kresja, J. L. Koenig, and A. B. Sullivan, Rubber Chem. TeChnol. 67, 348 (1994).

${ }^{35}$ M.H.S. Gradwell and W.J. McGill, J. Appl. Polym. Sci. 51, 169 (1994).

${ }^{36}$ M.H.S. Gradwell and W.J. McGill, J. Appl. Polym. Sci. 51, 177 (1994).

${ }^{37}$ M.H.S. Gradwell and W.J. McGill, J. Appl. Polym. Sci. 61, 1131 (1996).

${ }^{38}$ M.H.S. Gradwell and W.J. McGill, J. Appl. Polym. Sci. 61, 1515 (1996).

${ }^{39}$ R. T. Lieb, A. B. Sullivan, and C.D. Trivette, Rubber Chem. Technol. 43, 1188 (1970).

${ }^{40}$ P. N. Son, K.E. Andrews, and A.T. Schooley, Rubber Chem. Technol. 45, 1513 (1972).

${ }^{41}$ A. Y. Coran, Rubber Chem. TeChnol. 68, 351 (1995).

${ }^{42}$ A. B. Sullivan, L. H. Davis, and O. W. Maender, Rubber Chem. Technol. 56, 1061 (1983).

${ }^{43}$ B. A. Dogadkin, V. Selyukova, Z. Tarasova, A. Dobromyslova, M. Feldshtein, and M. Kaplunov, RubBER CHEM.

TeChNOL. 31, 348 (1958).

${ }^{44}$ B. A. Dogadkin, O. N. Beliatskaya, A.B. Dobromyslova, and M.S. Feldshtein, RubBer Chem. Technol. 33, 361 (1960).

${ }^{45}$ M. H. S. Gradwell, K. G. Hendrikse, and W.J. McGill, J. Appl. Polym. Sci. 72, 1235 (1999).

${ }^{46}$ M. H. S. Gradwell, B. Morgan, and W. J. McGill, J. Appl. Polym. Sci. 56, 1581 (1995).

${ }^{47}$ R. S. Kapur, J. L. Koenig, J. R. Shelton, Rubber Chem. Technol. 47, 911 (1974).

${ }^{48}$ F. Fairbrother, G. Gee, and G.T. Merrall, J. Polymer Sci. 16, 459 (1955).

${ }^{49}$ C. D. Trivette, J. E. Morita, and E.J. Young, RubBer ChEM. TeChnOL. 35, 1360 (1962).

${ }^{50}$ D. M. Gardner, and G. K. Fraenkel, J. Amer. Chem. Soc. 78, 3279 (1956).

${ }^{51}$ M. M. Coleman, J. R. Shelton, and J.L. Koenig, Rubber Chem. Technol. 46, 938 (1973).

${ }^{52}$ M. M. Coleman, J. R. Shelton, and J.L. Koenig, Rubber Chem. Technol. 46, 957 (1973). 
${ }^{53}$ J. R. Shelton, and E. T. McDonel, Rubber Chem. Technol. 33, 342 (1960).

${ }^{54}$ S. P. Manik, and S. Banerjee, Rubber Chem. TeChnol. 43, 1311 (1970).

${ }^{55}$ L. Bateman, C.G. Moore, and M. Porter. J. Chem. Soc. 2866 (1958).

${ }^{56}$ B. A. Dogadkin, V. Selyukova, Z. Tarasova, A. Dobromyslova, F. Feldshtein, and M. Kaplunov, RubBer ChEM.

TECHNOL. 29, 917 (1956).

${ }^{57}$ B. A. Dogadkin, J. Polym. Sci. 30, 351 (1958).

${ }^{58}$ B. A. Dogadkin, O. N. Beliatskaya, A.B. Dobromyslova, and M.S. Feldshtein, Rubber ChEm. Technol. 33, 361 (1960).

${ }^{59}$ M. Geyser and W. J. McGill, J. Appl. Polym. Sci. 55, 215 (1995).

${ }^{60}$ B. Morgan and W. J. McGill, J. Appl. Polym. Sci. 76, 1405 (2000).

${ }^{61}$ A. Muller and B. Krebs, in "Studies in Inorganic Chemistry," Elsevier, N.Y. (1984).

${ }^{62}$ J. A. Semlyen, Trans. Faraday Soc. 63, 743 (1967).

${ }^{63}$ R. Steudel, Z. Anorg. Allg. Chem. 478, 156 (1981).

${ }^{64}$ D. C. Koningsberger and T. Deneef, Chem. Phys. Lett. 4, 615 (1970).

${ }^{65}$ D. C. Koningsberger, Dissertation, Techn. Univ. Eindhoven (Netherlands), 1971.

${ }^{66}$ E. E. Ried in "Organic Chemistry of Bivalent Sulfur," Chemical Publishing Co., New York, 1960, vol. 3, p. 362.

${ }^{67}$ E. N. Guryanova, Q. Rep. Sulfur. Chem. 5, 113 (1970).

${ }^{68}$ F. Lamar in "Organic Chemistry of Sulfur," S. Oae, Ed., Plenum Press, N.Y., 1977, p. 303.

${ }^{69}$ P. J. Nieuwenhuizen, A. W. Ehlers, J.W. Hofstraat, S.R. Janse, M.W.F. Nielen, J.Reedijik, and E.J. Baerends,

Chem. - Eur. J. 4, 1816 (1998).

${ }^{70}$ W. H. Kruger and W. J. McGill, J. Appl. Polym. Sci. 42, 2643 (1991).

${ }^{71}$ W. H. Kruger and W. J. McGill, J. Appl. Polym. Sci. 42, 2661 (1991).

${ }^{72}$ W. H. Kruger and W.J. McGill, J. Appl. Polym. Sci. 42, 2669 (1991).

${ }^{73}$ M. G. Huson, W. J. McGill, and R. D. Wiggett, J. Polym. Sci., Polym. Chem. 23, 2883 (1985).

${ }^{74}$ B. Morgan and W.J. McGill, J. Appl. Polym. Sci. 76, 1386 (2000).

${ }^{75}$ H. Krebs, Rubber Chem. Technol. 30, 962 (1957).

${ }^{76}$ S. P. Manik and S. Banerjee, Rubber Chem. Technol. 43, 1311 (1970).

${ }^{77}$ E. Morita and E. J. Young, Rubber Chem. TeChnol. 36, 844 (1963).

${ }^{78}$ W. Scheele and A. Franck, Rubber Chem. Technol. 32, 566 (1959).

${ }^{79}$ C. G. Moore and A. A. Watson, J. Appl. Polym. Sci. 8, 581 (1964).

${ }^{80}$ A. M. Zaper and J. L. Koenig, Rubber Chem. TeChnol. 60, 278 (1987).

${ }^{81}$ M. R. Kresja and J. L. Koenig, Rubber Chem. Technol. 65, 427 (1992).

${ }^{82}$ A. M. Zaper and J. L. Koenig, Rubber Chem. TeChnol. 60, 252 (1987).

${ }^{83}$ A. M. Zaper and J. L. Koenig, Rubber Chem. TeChNOL. 60, 278 (1987).

${ }^{84}$ T. D. Skinner, Rubber Chem. Technol. 45, 182 (1972).

${ }^{85}$ L. Bateman, C.G. Moore, and M. Porter, J. Chem. Soc. 2866 (1958).

${ }^{86}$ F. K. Lautenschlaeger, RubBer ChEM. TeChNOL. 52, 213 (1979).

${ }^{87}$ F. K. Lautenschlaeger, Rubber Chem. Technol. 52, 1030 (1979).

${ }^{88}$ F. K. Lautenschlaeger, RUbBer CHEM. TeChNOL. 53, 27 (1980).

${ }^{89}$ L. S. Degtyarev and L. N. Ganyuk, Polymer Sci., USSR, 6, 31 (1962).

${ }^{90}$ B. A. Dogadkin and V. A. Shershnev, Rubber ChEM. TeChNOL. 33, 412 (1960).

${ }^{91}$ W. Scheele and O. Lorentz, Rubber Chem. TeChnol. 29, 29 (1956).

${ }^{92}$ W. Scheele and O. Lorentz, RubBer Chem. Technol. 29, 37 (1956).

${ }^{93}$ J. R. Wolfe, Jr., Rubber Chem. Technol. 41, 1339 (1968).

${ }^{94}$ A. Y. Coran in "Science and Technology of Rubber," E. F. R. Eirich., Ed. New York, Academic Press, 1978, ch. 7.

${ }^{95}$ A. Y. Coran, Chemtech 13, 106 (1983). 
${ }^{96}$ B. Dogadkin and L. Beniska, Rubber Chem. Technol. 31, 329 (1958).

${ }^{97}$ N. J. Morrison and M. Porter, RubBer Chem. Technol. 57, 63 (1984).

${ }^{98}$ B. Morgan and W. J. McGill, J. Appl. Polym. Sci. 76, 1395 (2000).

${ }^{99}$ A. Y. Coran, Rubber Chem. Technol. 38, 1 (1965).

${ }^{100}$ W. H. Kruger and W. J. McGill, J. Appl. Polym. Sci. 45, 573 (1992).

${ }^{101}$ W. Scheele, H. E. Toussaint, and Y. K. Chai, Rubber Chem. Technol. 34, 334 (1961).

${ }^{102}$ M. H. S. Gradwell and W. J. McGill, J. Appl. Polym. Sci. 61, 1515 (1996).

${ }^{103}$ C. G. Moore and B. R. Trego, J. Appl. Polym. Sci. 8, 1957 (1964).

${ }^{104}$ R. B. Layer, Rubber Chem. Technol. 65, 211 (1992).

${ }^{105}$ R. B. Layer, Rubber Chem. Technol. 65, 822 (1992).

${ }^{106}$ M. Andreis, J.Liu, J. L. Koenig, J. Polym. Sci., Polym. Phys. Ed. 27, 1389 (1989).

${ }^{107}$ A. A. Watson, Ph. D. Thesis, University of London (1965).

${ }^{108}$ C. J. Moore and B. R. Trego, Tetrahedron 18, 205 (1962).

${ }^{109}$ C. J. Moore and B. R. Trego, Tetrahedron 19, 1251 (1963).

${ }^{110}$ G. P. McSweeney and N. J. Morrison, Rubber Chem. Technol. 56, 337 (1983).

${ }^{111}$ M. G. Voronkov and E. N. Deryagina in “Chemistry of Organosulfur Compounds,” L. I. Belen'kii, Ed., Ellis Horwood, New York 1990, p. 48.

${ }^{112}$ I. Kende, T. L. Pickering, and A.V. Tobolsky, J. Am. Chem. Soc. 87, 5582 (1965).

${ }^{113}$ T. L. Pickering, J. Saunders, and A.V. Tobolsky, J. Am. Chem. Soc. 89, 2364 (1967).

${ }^{114}$ T. L. Pickering, K. J. Saunders, and A. V. Tobolsky in “The Chemistry of Sulfides,” A. V. Tobolsky, Ed., John Wiley \& Sons Inc., New York, 1968, p. 61.

${ }^{115}$ D. J. Martin, A. Drayer, M. Ropero, and M.A. Alonso, Int. J. Chem. Kinet. 14, 131 (1982).

${ }^{116}$ C. H. Chen, J. L. Koenig, J. R. Shelton, and E. A. Collins, Rubber Chem. Technol. 55, 103 (1982).

${ }^{117}$ A. M. Zaper and J. L. Koenig, Makromol. Chem. 189, 1239 (1988).

${ }^{118}$ A. B. Sullivan, E. Morita, and R. I. Leib, Rubber World, 195(1), 21 (1986).

${ }^{119}$ C. D. Trivette, and A. Y. Coran, J. Org. Chem. 31, 100 (1966).

${ }^{120}$ M.H.S. Gradwell, and N.R. Stephenson, RubBer ChEm. TeChnOL. 74, 44 (2001).

${ }^{121}$ R. T. Lieb, A. B. Sullivan, and C. D. Trivette, Rubber Chem. Technol. 43, 1188 (1970).

${ }^{122}$ M. Boudart, "Kinetics of Chemical Process," Butterworth-Heinemann Reprint Series in Chemical Engineering (1991), p. 167.

${ }^{123}$ A. Isihara, J. Phys. Soc. Jpn. 5, 201 (1950).

${ }^{124}$ P. Debye and F. Bueche, J. Chem. Phys. 20, 1337 (1952).

${ }^{125}$ P. J. Flory, "Statistical Mechanics of Chain Molecules," Hanser Publishers, New York (1989).

${ }^{126}$ M. Schmidt, Angew. Chem. Int. Ed. 12, 445 (1973).

${ }^{127}$ B. Meyer, Chemical Reviews 76, 367 (1976).

${ }^{128}$ R. Steudel, Angew. Chem. Int. Ed. 14, 655 (1975).

${ }^{129}$ J. A Semlyen, Trans. Faraday Soc. 63, 743 (1967).

${ }^{130}$ O. M. Suleimenov and Tae-Kyu Ha, Chem. Phys. Lett. 290, 451 (1998).

${ }^{131}$ A. Karpfen, Chem. Phys. Lett. 136, 571 (1987).

${ }^{132}$ P. Birner, H. J. Kohler, A. Karpfen, and H. Lischka, J Mol. Struct. (Theochem) 226, 223 (1991).

${ }^{133}$ J. E. Mark, J. Am. Chem. Soc. 88 (1966).

${ }^{134}$ P.J. Flory, "Principles of Polymer Chemistry," Cornell University Press, Ithaca, NY (1953).

${ }^{135}$ D. W. Marquardt, J. Soc. Ind. Appl. Math. 11, 431 (1963).

${ }^{136}$ A. B. Sullivan, C. J. Hann, and G. H. Kuhls, Rubber Chem. Technol. 65, 488 (1992). 
${ }^{137}$ B. Morgan and W. J. McGill, J. Appl. Poly. Sci. 76, 1413 (2000).

${ }^{138}$ P. Ghosh, "A Systematic Framework for Computer-Aided Design of Engineering Rubber Formulations," PhD Thesis, Purdue University, West Lafayette, IN (2002).

${ }^{139}$ S. Vajda, P. Valko, and T. Turyani, Int. J. Chem. Kinet. 17, 55 (1985).

${ }^{140}$ Y. Bard, "Nonlinear Parameter Estimation,” Academic, NY (1974).

${ }^{141}$ R. McWeeny and B. T. Sutcliffe, "Methods of Molecular Quantum Mechanics," Academic Press, NY (1989).

[ Received April 2003 ] 